\title{
Neuronal correlates of implicit learning in the mammalian midbrain
}

\author{
PhD Thesis \\ for the award of the degree \\ Doctor of Philosophy \\ in the Neuroscience Program \\ at the Georg-August-Universität Göttingen, \\ Faculty of Biology
}

submitted by

Hugo Cruces Solís

born in

Mexico City, Mexico

Göttingen, 29 ${ }^{\text {th }}$ February 2016 



\section{Thesis Committee}

Dr. Livia de Hoz, Supervisor

Department of Neurogenetics

Max Planck Institute of Experimental Medicine, Göttingen

Prof. Dr. Klaus-Armin Nave, Reviewer

Department of Neurogenetics

Max Planck Institute of Experimental Medicine, Göttingen

Prof. Dr. Tobias Moser, Reviewer

Institute for Auditory Neuroscience and InnerEarLab,

University Medical Center, Göttingen

Prof. Dr. Stefan Treue

Cognitive Neuroscience Laboratory,

German Primate Center, Göttingen

\section{Further members of the Examination Board}

Prof. Dr. Siegrid Löwel

Department of Systems Neuroscience, Bernstein Focus Neurotechnology,

Johann-Friedrich-Blumenbach Institute for Zoology and Anthropology, University of Göttingen, Göttingen

Dr. Andreas Neef

Bernstein Group Biophysics of Neural Computation,

Max Planck Institute for Dynamics and Self-Organization, Göttingen

Prof. Dr. André Fischer

Department for Psychiatry and Psychotherapy

University Medical Center

Date of oral examination: April $26^{\text {th }}, 2016$ 


\section{CONTENTS}

ABSTRACT

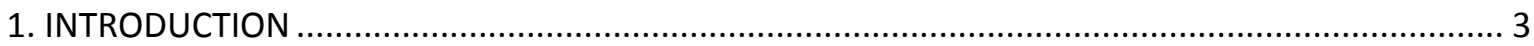

1.1 Experience-dependent plasticity in the auditory system.................................................. 4

1.1.1 Cortical experience-dependent plasticity by sound exposure during early postnatal

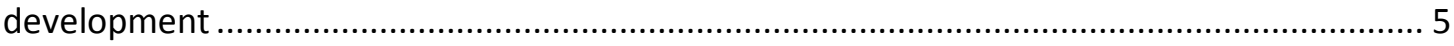

1.1.2 Cortical experience-dependent plasticity by sound exposure during adulthood............. 6

1.1.3 Auditory cortical experience-dependent plasticity induced by associative learning........ 8

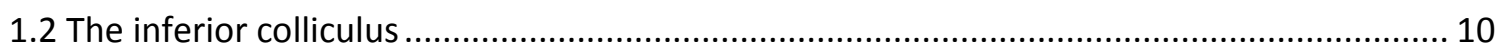

1.2.1 Collicular experience-dependent plasticity by sound exposure during early postnatal development

1.2.2 Collicular experience-dependent plasticity by associative learning during adulthood and the role of the cortico-collicular projections......................................................................... 16

1.2.3 Plasticity in the auditory midbrain. Studies in humans.............................................. 16

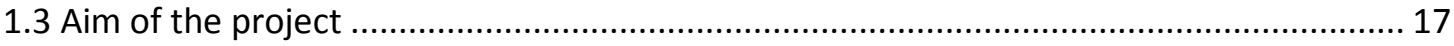

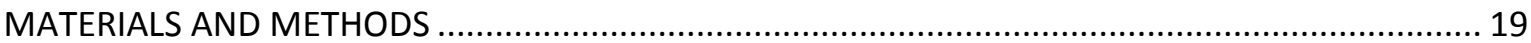

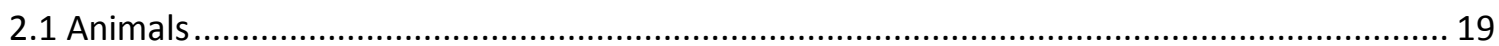

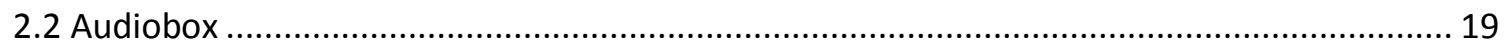

2.3 Sound exposure

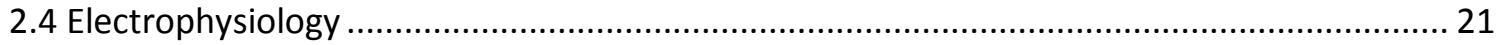

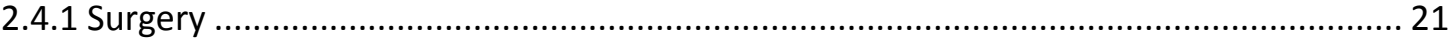

2.4.2 Acoustic stimulation during electrophysiological recordings ........................................ 23

2.4.3 Simultaneous cortical inactivation and collicular recording ....................................... 24

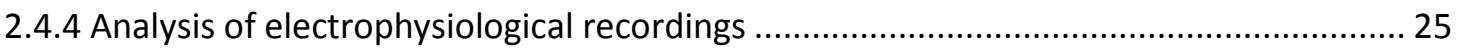

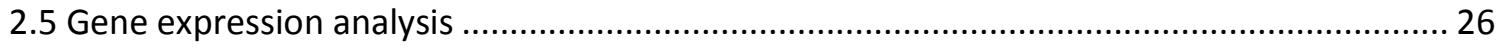

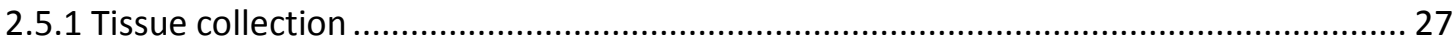

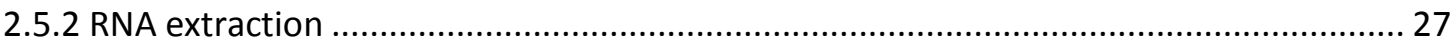

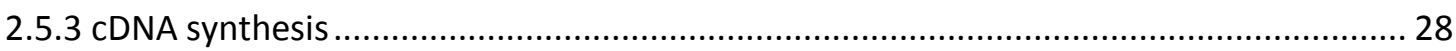




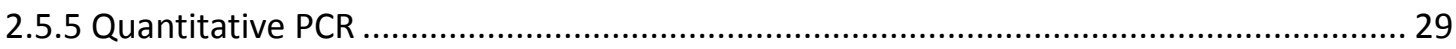

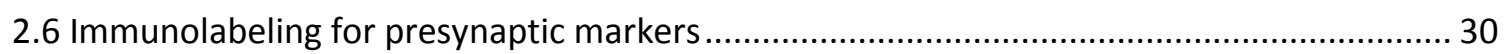

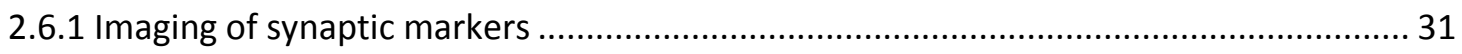

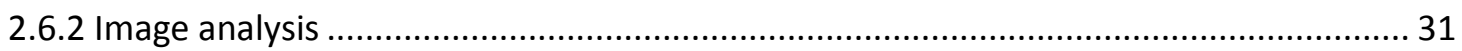

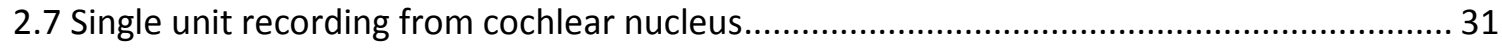

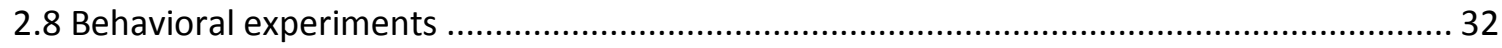

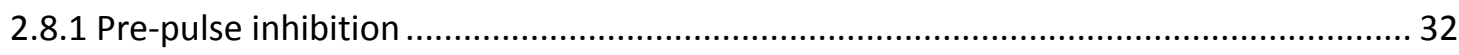

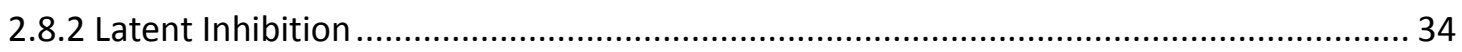

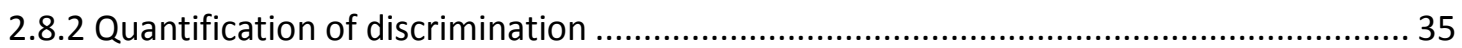

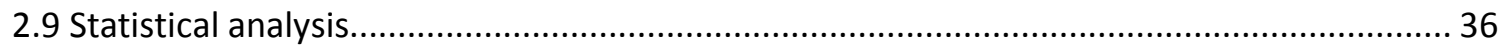

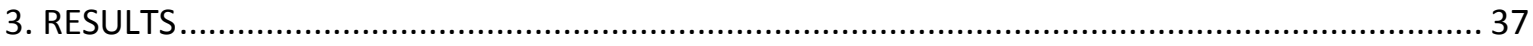

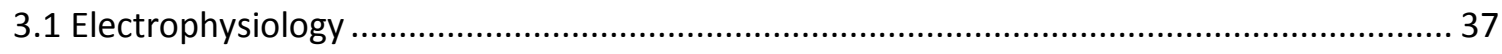

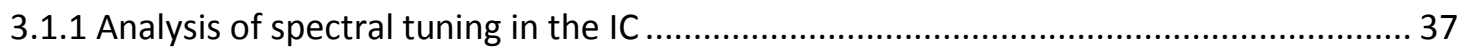

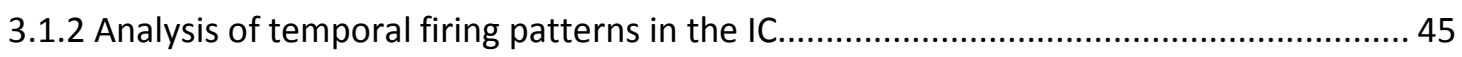

3.1.2 Two-dimensional organization of frequency representation in the IC ......................... 51

3.1.3 Effect of days of sound exposure on collicular plasticity ...........................................53

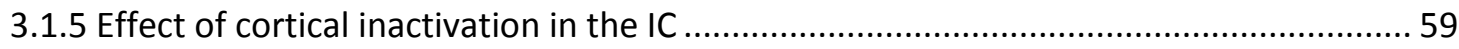

3.1.6 Analysis of spectral tuning in the lateral cortex of the IC ............................................... 64

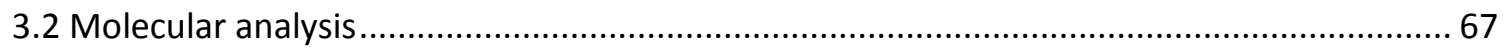

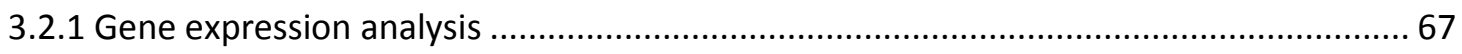

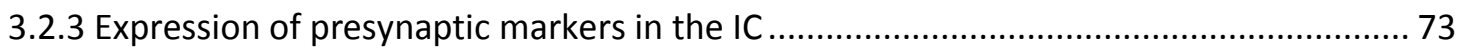

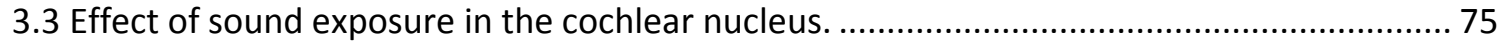

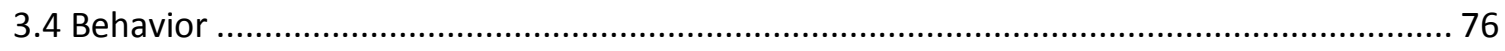

3.4.1 Pre-pulse inhibition of the acoustic startle response. ................................................. 76

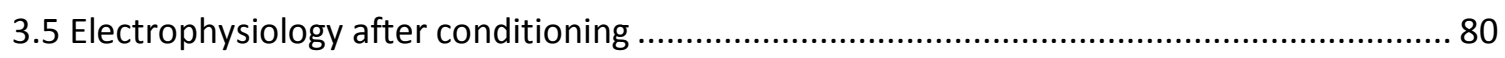

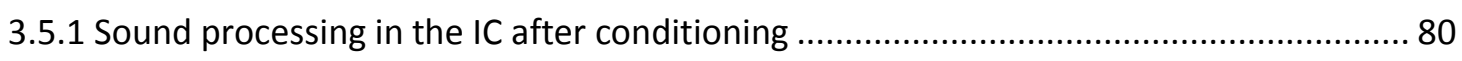

3.6 Regulation of auditory cortical plasticity by blocking c-Fos expression................................ 85 


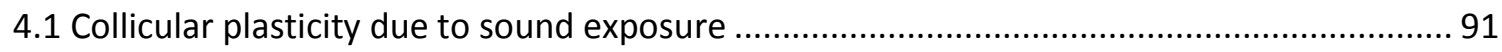

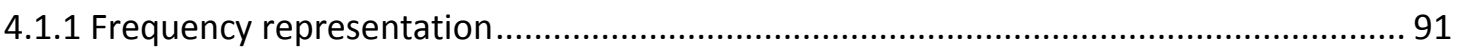

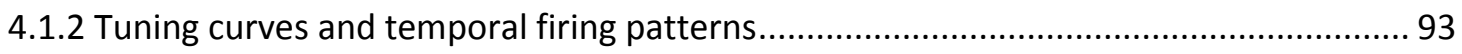

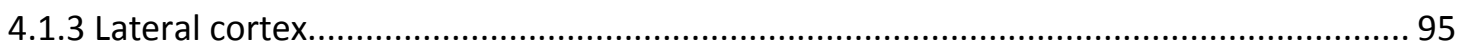

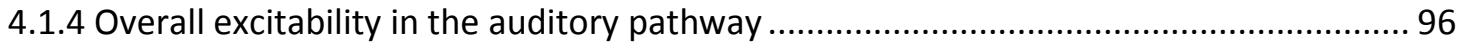

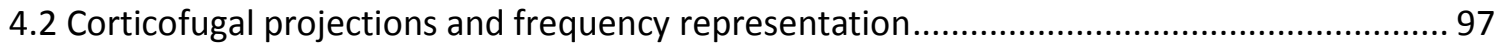

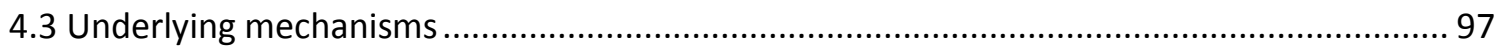

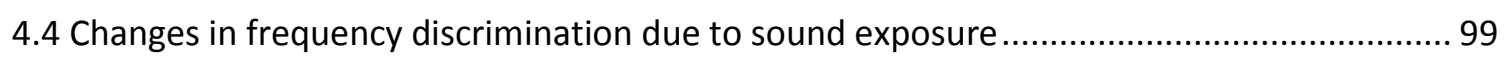

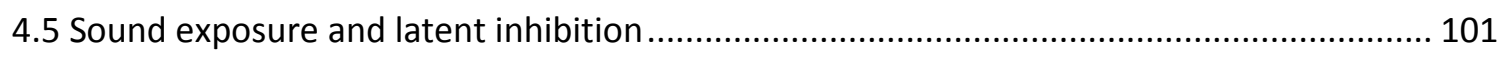

4.5.1 Collicular plasticity due to conditioning ................................................................. 103

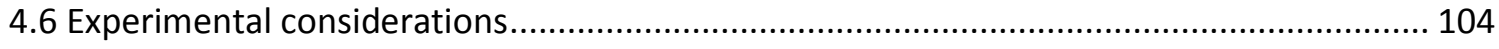

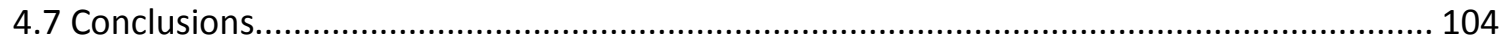

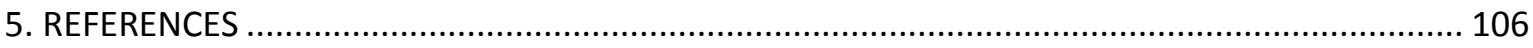

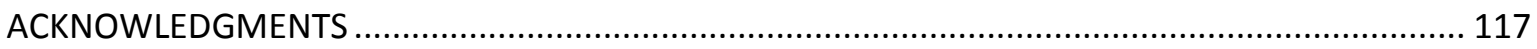

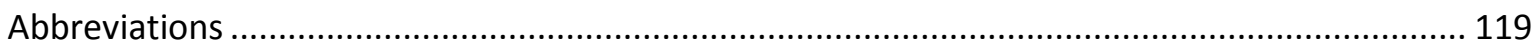

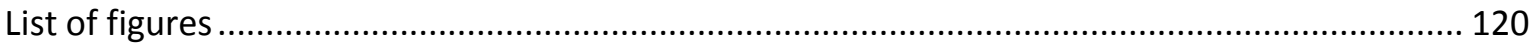

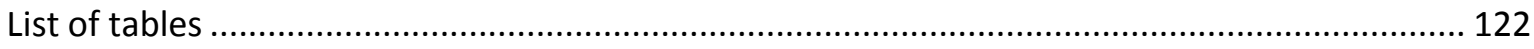

CURRICULUM VITAE 


\section{ABSTRACT}

Filtering auditory information according to its relevance is critical to elicit the appropriate behavioral response. The relevance of a sound is not carried by the sound itself but rather assigned by the brain based on previous experience to the same or similar sounds. Although in experimental settings, the effects of learning on sound processing are mostly studied in the context of extensive training, in real life the value and meaning of many sounds is learned without explicit feedback (implicit auditory learning). Up to date, it is not clear at which level of the auditory pathway previous experience starts to contribute to sound processing of incoming information. The inferior colliculus (IC), located in the midbrain, is the first auditory nucleus in the auditory pathway where inputs from all ascending and several descending auditory nuclei converge. Moreover, it also receives projections from multiple non-auditory areas, which suggests that it can be modulated by multiple factors. Here, using a combination of behavioral, electrophysiological and molecular tools, I tested the hypothesis that already at the level of the IC the sensory input is influenced by implicitly learned auditory associations.

To manipulate the auditory experience of animals I used the Audiobox. The Audiobox is an automated testing chamber, where mice live 24 hours a day, allowing continuous monitoring of behavior. It consists of two compartments: a home cage and a sound - attenuated corner with water access, separated by a long corridor. To drink, mice needed to visit the corner and the individual visits were detected by a transponder previously implanted in each mouse. In a group of mice, every visit to the corner was paired with the presentation of tone pips of a specific frequency (exposed group). The control group consisted of mice that lived in the Audiobox, but were not exposed to sound in any compartment. In the exposed group, the sound was paired with a specific action in a specific area (visits to the corner), hence it was the group where an implicit association could develop. I characterized the evoked responses from multinunit activity in the IC by performing acute electrophysiological recordings in anesthetized mice. I found that, after 6-12 days of sound exposure, the amplitude of the tuning curves were higher than the control group, also there was a unspecific reorganization in frequency representation. There was also an expansion of the area that responded to the frequency used during behavior. These changes were not due to an increase in the overall excitation in the auditory pathway, since no changes in sound processing were found in the cochlear nucleus. It has been shown that collicular plasticity depends on cortical feedback. However, recordings in the IC while simultaneously inactivating the cortex revealed that no cortical feedback is needed for the maintenance of the observed changes. The electrophysiological changes were paralleled at a molecular level with an increase in the excitation/inhibition ratio in collicular synapses, as measured by immunolabeling of VGAT and Vglut2. 
To test the effects of sound exposure alone, without implicit learning, I performed recordings in a group of animals that lived in the Audiobox, and were exposed to the same sound but in a random way. This group also showed plasticity in the IC, also in the form of tuning curves of larger amplitude. However these changes were more dominant in the dorsal cortex of the IC, an area that did not show plasticity in the exposed group. The shift in frequency representation was visible in this group but smaller than the induced in the exposed group. Additionally, it did not show an expansion of the area responsive to the exposed sound.

A key question is whether these plastics changes, induced by implicit learning, had an effect on subsequent behavioural responses or even learning. To test whether frequency discrimination at a behavioral level could be affected by the changes described in sound processing in the IC, I tested frequency discrimination acuity using pre-pulse inhibition of the acoustic startle response, whose expression strongly depends on the IC. Sound exposure decreased frequency discrimination acuity in the exposed group, but not in the random group, indicating that relevant sound exposure, unlike random, increased sound generalization. To test implicit auditory learning, I trained the animals in a two-tone discrimination sound, where the conditioned sound had been previously presented in a non-conditioned manner. The exposed group elicited latent inhibition, a delay in learning, while the random group learned the task within the first day, indicating that indeed, the exposed group had developed an association between the exposed sound and a neutral outcome, previous to conditioning.

Together, these results strongly support the idea of a correlation between long-term collicular plasticity of sound processing and two behavioral readouts of frequency discrimination, supporting the theory that the IC is a subcortical filter of current auditory information that is adjusted by previous auditory experience. Implicit auditory learning has been related to the developing of important communication processes such as the categorization of phonemes. The work of the present thesis offers an animal model to study the neuronal correlates of implicit auditory learning and, in combination with genetic models of neurodevelopmental diseases, can contribute to the better understanding of the neuronal deficits underlying higher cognitive processes such as speech acquisition. 


\section{INTRODUCTION}

Survival of any organism critically depends on its ability to adequately sense and interpret the environment. In mammals, sensory systems are dedicated to transform some of the features of the external world into a neuronal code. Each sensory system is defined by the set of features it detects. For example, the auditory system detects mechanical vibrations that propagates through an elastic medium (sounds), the visual system detects electromagnetic waves (light) and the olfactory system detects volatile molecules (odors). Within each sensory system, the range of detectable features is optimized to the specific environment of each given species. However, the anatomical organization of the sensory systems is well conserved across species: they consist of similar sensory epithelia and related neuronal structures. Within this structures, the topographic order of the sensory epithelia is maintained in an organized manner: neurons tuned to similar features are grouped close to each other (topographic sensory representations). For example, topographic representations of the retina, cochlea and skin have been described along their respective anatomical pathways, including primary cortices (Bednar \& Wilson 2015; Wilson \& Bednar 2015; Kanold et al. 2014).

Sensory representations are not fixed but rather they adapt to environmental changes. A critical process that allows this adaptation is experience-dependent plasticity. Experience-dependent plasticity is defined as the ability of neurons to undergo functional and structural changes in response to either sensory input alone or sensory-motor loops. Probably the most dramatic adaptation of sensory representations occurs during critical periods. Critical periods are time windows in early postnatal neuronal development, in which sensory stimuli have an enhanced capacity to induce plastic changes in sensory systems (Katz \& Shatz 1996; Berardi et al. 2000; Sale et al. 2014). Specifically, neurons will bias their connections and response properties to represent the individual's external world. One of the most striking examples of plasticity during this period is the easiness with which children acquire any "mother" language they are exposed to. It is clear that this ability is absent for the majority of adult people (Kuhl 2010).

Once the critical period is over, the classic view is that, during adulthood, the experience needed to induce plasticity of sensory representations should be related to either important natural events such as motherhood; or to learning-related processes, such as associative or operant learning. In associative learning, also referred to as classical conditioning, a conditioned stimulus (CS, e.g. a sound) is paired with an unconditioned stimulus (US, e.g. usually a mild electric shock), in such a way that an association is generated. This association can be tested behaviorally by measuring the reaction of the animal to the CS alone. In contrast, in operant learning, the animal must learn that a specific sensory stimulus predicts a reward, if stimulus presentation is followed by a specific and voluntarily action (Blake et al. 2006; Bao 2015). However, in real life many stimuli are associated 
without the need of an action, be it innate or voluntary, from the animal. These implicit learning can affect subsequent responses to other related stimuli (De Houwer 2009; Vlahou et al. 2012). Implicit sensory learning is defined as the ability of a sensory stimulus to acquire behavioral relevance that is not derived from extensive associative or perceptual training. The ability of implicit learning to modify sensory representations during adulthood and its effect on subsequent learning has been barely explored and is the topic of the present thesis.

Experience-dependent plasticity during adulthood has been mainly related with cortical processes (Lee \& Whitt 2015; Medini 2014; Weinberger 2004; Kato et al. 2015). It is unlikely that plasticity during adulthood will generate long-term changes in the auditory epithelia, but whether it is a phenomenon that can be observed already in subcortical structures and the conditions under which this would be the case are poorly understood questions.

The current doctoral project aims to understand better the interaction between memory and sensation in the auditory pathway: how the past experience of a particular auditory stimulus will affect the way this and related acoustic stimuli are sensed. The assumption is that to filter auditory information efficiently, there should be an interaction -somewhere along the auditory pathwaybetween the ascending acoustic information about a specific stimulus and its learned and expected behavioral relevance or meaning.

In this work, the rodent auditory system is used as a model since it has been widely used to study the modifications of sensory representations during adulthood by different forms of learning (Weinberger 2015). Additionally, the auditory system possesses features that facilitate the experimental setup: it is constantly analyzing the soundscape, sounds are perceived from long distances and different directions (we heard what is behind us), and does not require directed attention, posture or fixation of any type (such as head movements or saccades in the visual system). In the following sections, cortical plasticity in the auditory system is described in more detail. Many studies explore cortical plasticity and their results have determined how we think about plasticity in general. This will be followed by a discussion of the few studies on subcortical experience-dependent plasticity, with emphasis in the inferior colliculus. Finally the scope of the thesis is stated.

\subsection{Experience-dependent plasticity in the auditory system}

Most studies of experience-dependent plasticity have been done in the auditory cortex. These are briefly reviewed here. 


\subsubsection{Cortical experience-dependent plasticity by sound exposure during early postnatal development}

In the core fields (primary auditory cortex and anterior auditory field) of the auditory cortex (AC), frequency representation is a two dimensional map along the cortical surface area (Figure 1.1). Primary auditory cortex (A1) is located in the caudal part of the AC and the anterior auditory field (AAF) is located in the rostral part. High frequencies are represented in the adjacent areas of A1 and $A A F$ and lower frequencies are represented more rostrally in the AAF and more caudally in A1 (Merzenich et al. 1975; Stiebler et al. 1997; Guo et al. 2012; Hackett et al. 2011; Kato et al. 2015). This characteristic gradient of frequency representation has been reported in at least 20 different mammalian species (Kaas 2011). In mice the measured range of cortical frequency representation in these areas is 2-64 kHz (Stiebler et al. 1997; Guo et al. 2012).

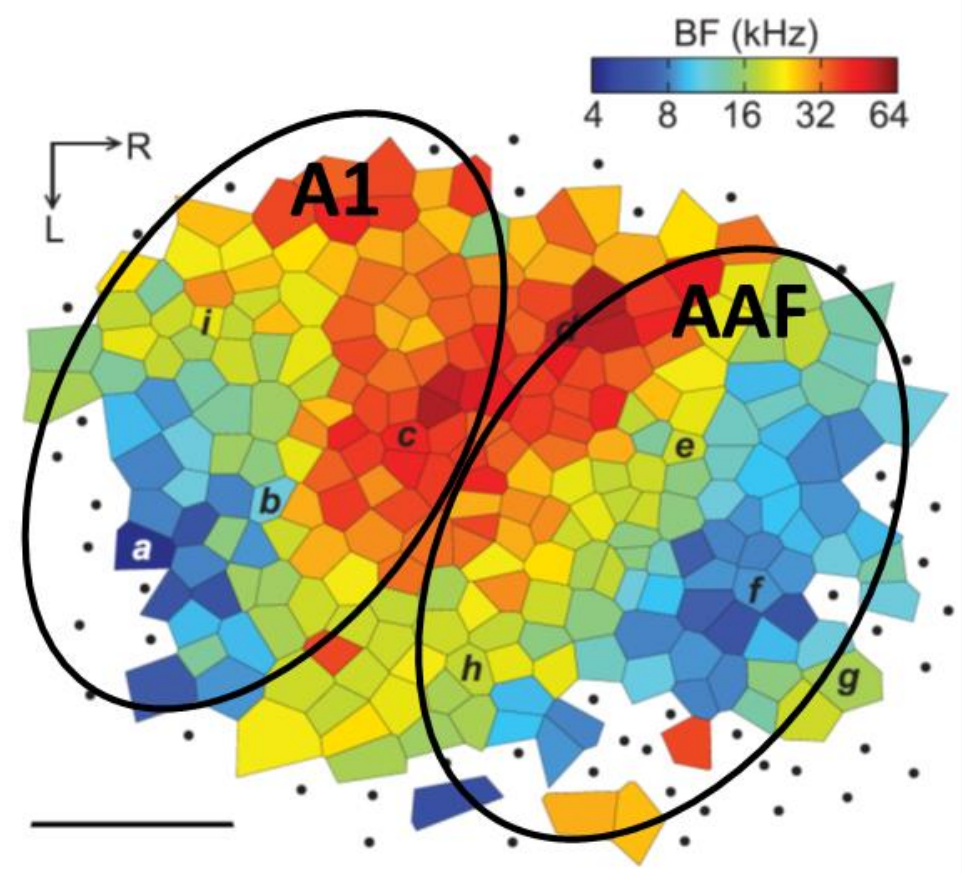

Figure 1.1: Frequency representation in the auditory cortex. Scheme of the tonotopic map of the core fields auditory cortex of the mouse. A1, primary auditory cortex; AAF, anterior auditory field. Scale bar: $0.5 \mathrm{~mm}$ (scheme modified from Guo et al 2012).

During early postnatal development (P11-P25 in rats), there is a gradual refinement of frequency selectivity of neurons in A1, that culminates with a tonotopic map that evenly covers the hearing range (Zhang et al. 2002; de Villers-Sidani et al. 2007). The influence of sound exposure on auditory cortical representations has been widely studied during the critical period. Sound exposure is achieved by placing speakers that deliver a sound, close to the standard cages where 

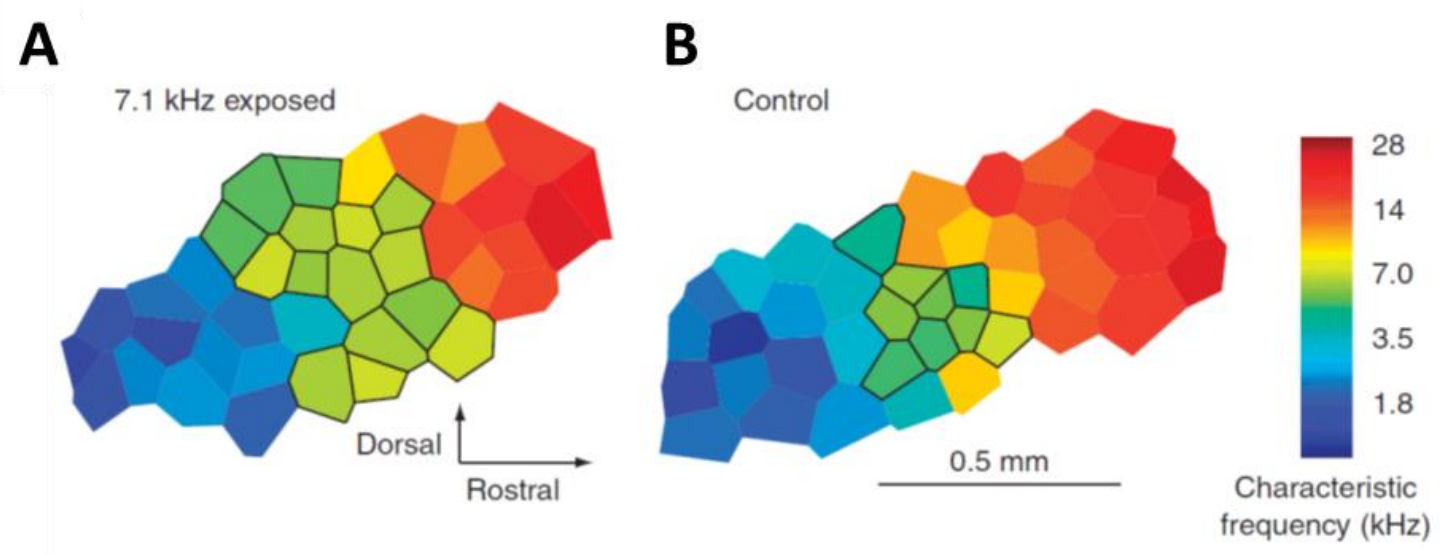

Figure 1.2: Modification of cortical sound representation. Scheme of the tonotopic map of the auditory cortex of example adult rats that were $\mathbf{A}$, exposed to $7.1 \mathrm{kHz}$ during the critical period were or B, reared under normal conditions (scheme taken from Han et al 2007).

the experimental subjects live (passive exposure). Extracellular recordings in A1 of anesthetized rats, revealed that long periods (10-16 hours per day) of passive exposure to one tone (monotonal) from postnatal day 8 (P8) to P28, leads to an overrepresentation of the exposed frequency that persists until adulthood when compared to control animals (Zhang et al. 2001). This manipulation of the cortical auditory representations leads to behavioral consequences during adulthood. Rats reared under monotonal exposure from P9-P30, failed to discriminate at P60 trains of repetitive tones from trains that contained two different tones of alternating frequencies near the exposed frequency (Figure 1.2) (Han et al. 2007).

Passive exposure to sounds that contain many frequencies with equal intensities (white-noise) delays the closure of the critical period (Chang \& Merzenich 2003). Rats exposed to white-noise from P8-P50 showed a degraded tonotopic map, characterized by incomplete frequency representation and wide tuning curves, resembling the maps at P16. Interestingly, rearing under normal sounds right after white-noise exposure, recovers the normal organization of A1. Additionally, white-noise exposure until P50 followed by monotonal exposure until P130 generated an overrepresentation of the exposed frequency.

These studies highlight the importance of the acoustic environment during early neuronal development, its long-term impact on cortical sound representations and the behavioral consequences of altered tonotopic maps.

\subsubsection{Cortical experience-dependent plasticity by sound exposure during adulthood}

Monotonal passive exposure during adulthood has no long-term impact on cortical frequency representation or neuronal responses to sounds (Chang \& Merzenich 2003; Zhou et al. 2011; Zhou \& Merzenich 2012; Whitton et al. 2014). On the other hand, passive exposure to a multiple range 
of tones, $(5-20 \mathrm{kHz})$, presented constantly in a random way for $\sim 120$ days, profoundly modifies cortical neuronal responses in anesthetized cats (Noreña et al. 2006). When compared to control animals, the neuronal responses of the exposed frequencies were reduced, while the frequencies below and above the exposed range were enhanced. These changes reversed following 12 weeks of rearing under quiet conditions (Pienkowski \& Eggermont 2009). These results make evident the different effects of passive exposure during and after the auditory critical period. It seems that during adulthood, more complex sounds and/or longer exposure times are required for passive exposure to affect cortical sound representations.

Passive exposure to white-noise degrades cortical tuning, synchronicity, temporal processing and behaviorally impairs the discrimination of high temporal sound rates in rats and cats (Gourévitch et al. 2014; Zhou \& Merzenich 2012). Adult rats exposed to white-noise for 30 days showed degraded cortical tonotopy and performed worse than control animals in a fine frequency discrimination task. However, they performed similarly when tested under noisy conditions while control animals performed worse than noise-exposed rats (Zheng 2012). These results suggest an effect of prior experience on modulating perceptual adaptation under noisy conditions. Similar experiments to explore changes in subcortical auditory areas have not been performed.

The effect of passive sound exposure in combination with enriched living conditions that favor cognitive and motor stimulation (e.g. bigger cages, larger groups of animals, running wheels, toys, etc.) has also been explored (environmental acoustic enrichment, EAE). In auditory cortex, EAE conditions increase response strength, synchronicity, reduces latencies and increases sharpening of tuning curves. The sounds presented were complex, included music and covered the hearing range of the rat (1-45 kHz) (Engineer 2004; Moucha et al. 2005; Percaccio et al. 2005; Kilgard et al. 2007; Percaccio et al. 2007). Interestingly, these changes were followed by an enhancement of glutamatergic synaptic currents measured in vitro (Nichols et al. 2007). These changes suggest that the interaction between sound and environment, unlike what happens during passive exposure, might trigger the release of neuromodulators. In support of this theory, it has been shown that pairing of passive acoustic stimulation with electrical stimulation of the nucleus basalis (a cholinergic nucleus) induces cortical frequency reorganization (Kilgard \& Merzenich 1998). Recently, it has been shown that rearing periods under EAE during adulthood, can rescue the physiological and behavioral deficits followed by noise exposure during early developmental stages (Zhu et al. 2014) or noise trauma (Norena \& Eggermont 2005), contributing to the evidence of the positive effects of enriched environment after brain insults (Greifzu et al. 2014). 


\subsubsection{Auditory cortical experience-dependent plasticity induced by associative learning}

Classical conditioning results from the pairing of a conditioned (CS) with unconditioned stimulus (US), which usually consists of a mild electric shock. The effects of classical conditioning on primary auditory cortex were first described in 1956 (Galambos, Robert Sheatz, Guy Vernier 1956), who measured evoked potentials in cats before and after conditioning, using clicks as the CS. Conditioning induced an increase in the amplitude of the evoked potentials in the auditory cortex and the cochlear nucleus. The classic work of Bakin and Weinberger (1990) demonstrated that in neurons of the auditory cortex of awake guinea pigs, classical conditioning produces a specific increase in the responses to the conditioned sound and a reduced response to the original best frequency (BF) (Figure 1.3). This difference generates a shift in the tuning towards the conditioning tone. On the other hand, the unpaired presentation of the CS and the US does not generate a behavioral conditioned response and, in the AC, induces a generalized non-specific increase in evoked responses, without a tuning shift, suggesting that only associative learning induces cortical tuning shifts (Weinberger 2004).

Perceptual or operant learning can induce reorganization of the cortical auditory representations specific for the feature of the target stimulus that the animals need to discriminate. For example, a frequency discrimination task can induce overrepresentation of the target frequency in monkeys and rats (Recanzone et al. 1993; Blake et al. 2002; Polley et al. 2006), while an intensity discrimination task induces an increase in the proportion of neurons tuned to the target intensity also in rats (Polley et al. 2004; Polley et al. 2006) and a sound-repetition rate discrimination task induces stronger phase-locking responses indicating a better temporal sound processing (Bao et al. 2004; Zhou \& Merzenich 2009; Zhou et al. 2015). Similar cortical modifications have been observed in humans (Morris et al. 1998; Mears \& Spencer 2012; Kluge et al. 2011; Whitton et al. 2014) suggesting a common adaptive mechanism. Additionally, it has been shown that the performance during the task influences the size of the cortical expansion to the target frequency (Rutkowski \& Weinberger 2005). Rats with different levels of water deprivation were trained to perform a frequency discrimination task to obtain water reward. The different degrees of water deprivation generated different degrees of performance. Cortical auditory maps were obtained after training. Interestingly, there was positive correlation between the performance and the size of the cortical representation of the target frequency, which strongly suggested that changes in cortical auditory representations directly contribute to the behavioral performance (Bao 2015). 


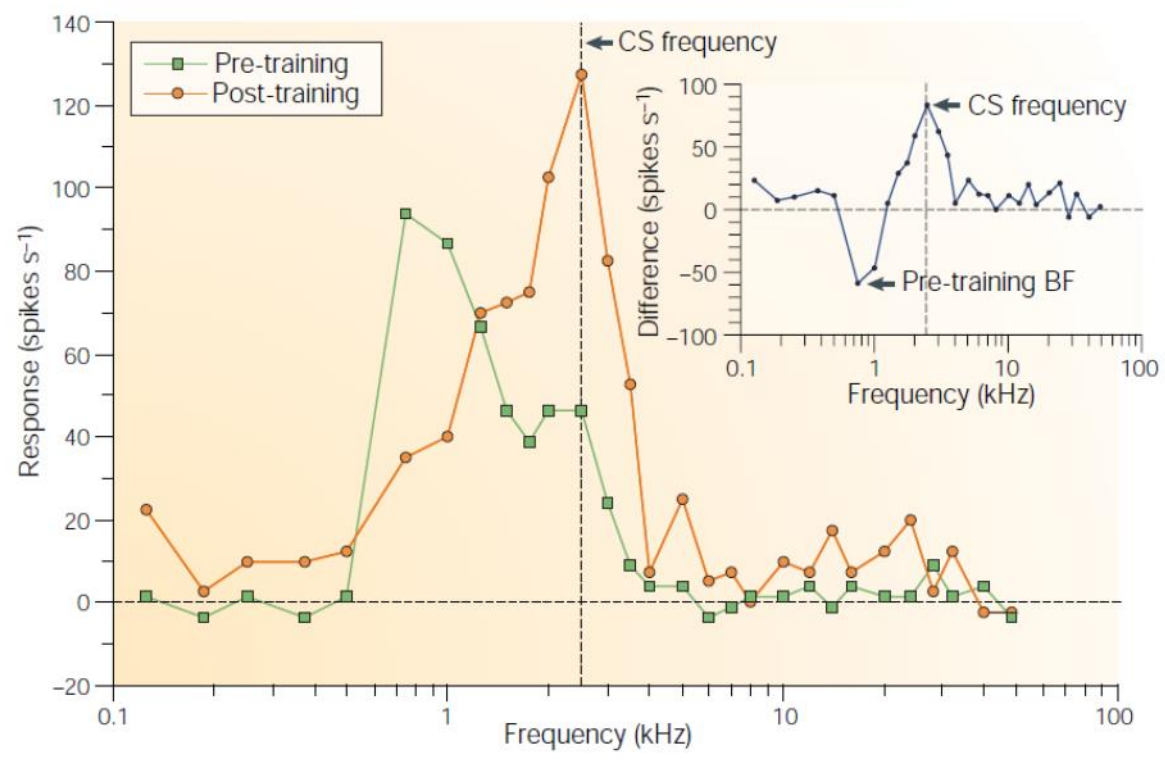

Figure 1.3: Receptive field plasticity in cortical neuron. Tuning curve of a single neuron from the auditory cortex of the gerbil before (green) and after conditioning learning (orange). The inset represents the difference in the spike rate of the conditioned tone before and after conditioning (scheme taken from Weinberger 2004).

What are the mechanisms that drive cortical long-term changes in auditory cortical representations? There is evidence that neuromodulatory transmitters, such as acetylcholine and dopamine, play an important role to signal behavioral relevance of a sensory input and drive the remodeling of cortical representations towards the relevant acoustic input. Pharmacological experiments have shown that cortical application of the cholinergic antagonist atropine prevents the frequency-specific plasticity induced by associative learning (Bakin \& Weinberger 1996; Ji \& Suga 2003). Conversely, tone exposure paired with electrical stimulation of the cholinergic nucleus basalis induces overrepresentation of the exposed tone and sharpening of the tuning curves in a similar way as perceptual training (Kilgard \& Merzenich 1998). Changes in the receptive field of cortical neurons, under this experimental paradigm, are generated by a fast reduction in the inhibitory synaptic input and an increase in the excitation that is specific to the exposed tone (Froemke et al. 2007). This artificial retuning of cortical neurons improved sensory perception at the frequency and intensity of the paired stimulus (Froemke et al. 2013). Rats learned to discriminate a target tone at any intensity $(4 \mathrm{kHz})$ from other tones. The performance was better for louder target tones ( $\sim 50 \mathrm{~dB} \mathrm{SPL}$ ). After pairing tone stimulation (4 $\mathrm{kHz}$ at $30 \mathrm{~dB} \mathrm{SPL}$ ) with electrical stimulation of the nucleus basalis or infusion of the cholinergic agonist carbachol directly in $A 1$, the performance for lower intensities of the target tone improved. Moreover, infusion of atropine or the NMDA receptor antagonist AP5 prevented the improvement of the performance 
induced by nucleus basalis stimulation, suggesting that long-term synaptic modifications in A1 are crucial for the improvement of sensory perception.

Dopamine plays an important role in reward signaling (Bermudez \& Schultz 2014; Schultz et al. 2015). It has been hypothesized that dopaminergic nuclei such as the ventral tegmental area (VTA) provide reinforcement signals that might drive changes in cortical representations that follow perceptual training. Pairing tone exposure with electric stimulation of the VTA, in a similar way as with previous experiments involving NB, induced an expansion of the cortical area that represents the exposed tone and improved the synchronicity between cortical neurons (Bao et al. 2001). Additionally, VTA stimulations reduces the variability and shortens the responses of cortical neurons (Lou et al. 2014).

Thus, important requisites for a sensory neuronal structure to undergo experience-dependent plasticity seem to be: a high degree of convergence of sensory input that allows for complex processing of incoming information and, at the same time, a high degree of connectivity with multiple other brain regions, such as neuromodulatory or associative nuclei, to be able to integrate the behavioral states and the relevance of the sensory input. The auditory cortex has proven to be the ideal candidate that fulfills these criteria. However, in the auditory pathway, the auditory cortex is not the only structure that possess a high degree of connectivity. The inferior colliculus (IC), located in the midbrain, also fulfills these criteria and makes it an ideal candidate to change upon relevant experience and modulate the incoming auditory information before it reaches the cortex.

\subsection{The inferior colliculus}

The IC is located in the posterior part of the midbrain tectum, has the highest metabolic rate in the brain (Zeller et al. 1997) and is the first auditory integration center where connections from all the auditory structures converge. It receives projections from all the auditory nuclei in the brainstem: cochlear nucleus, superior olivary complex and nucleus of the lateral lemniscus (Figure 1.4). Due to this convergence, important features of sound processing emerge here, such as critical bands, while others that 


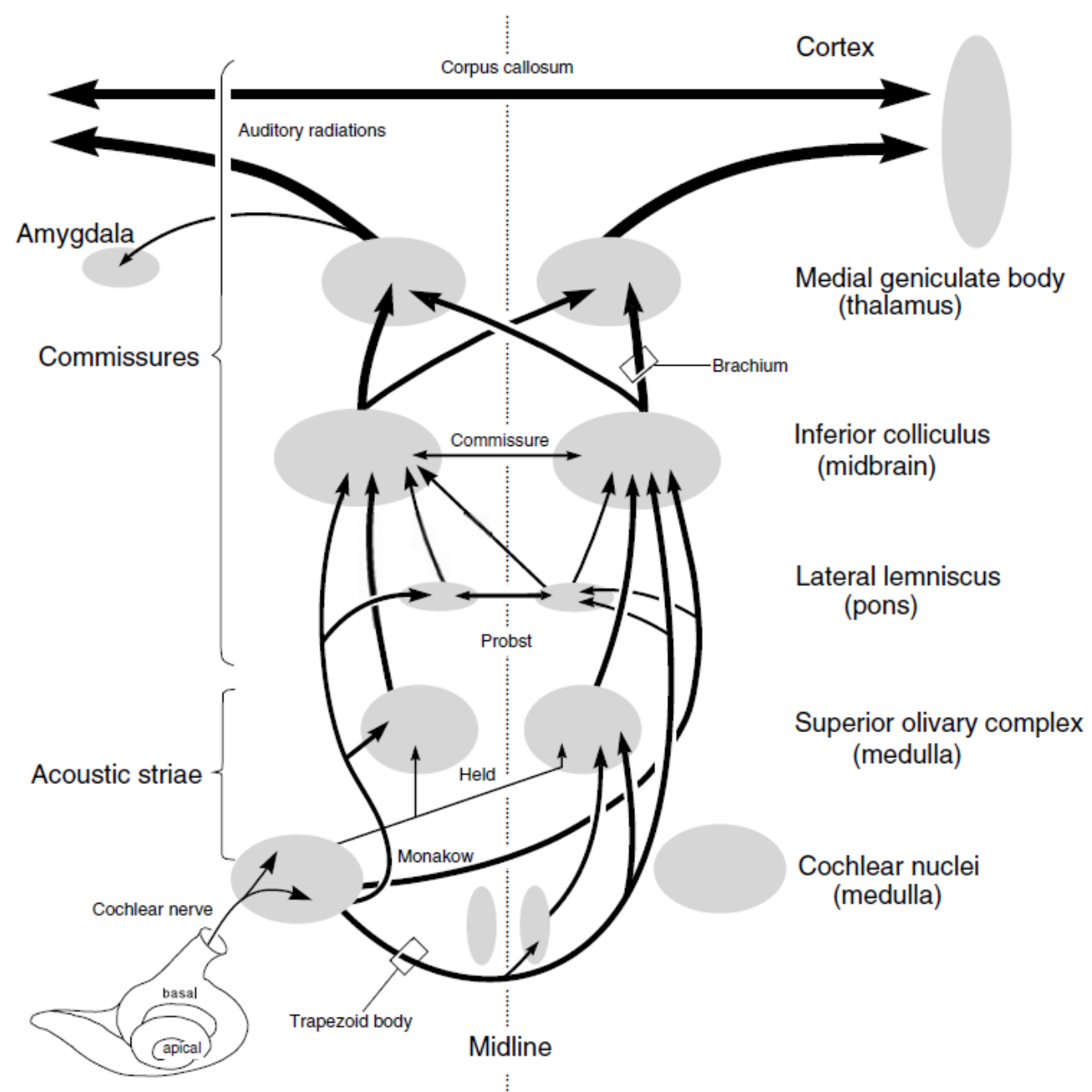

Figure 1.4: The auditory pathway. Scheme of the ascending connections and different nuclei of the central mammalian auditory pathway (scheme taken from Winer \& Schreiner 2005).

emerged earlier are refined, such as binaural coding (Ehret \& Schreiner 2005; Ehret, Günter Merzenich 1985; Schreiner et al. 1997; Palmer \& Kuwada 2005). The IC contains an important number of commissural fibers that connect both colliculi, it sends reciprocal projections to the brainstem nuclei that target it and bilateral projections to the thalamus, indicating a high degree of interconnectivity and feedback loops. It also receives projections from non-auditory areas, including somatosensory, amygdaloid and neuromodulatory regions suggesting an important role as both a multisensory processing center and a modulatory structure (Winer 2005). The IC receives an important amount of cortical projections that arise from layer $V$ and to lesser degree from layer VI whose physiological role in the context of experience-dependent plasticity will be discussed in more detail below.

The IC is divided into three main nuclei: the central nucleus, lateral nucleus or lateral cortex and dorsal cortex (Figure 1.5B). The central nucleus (ICC) is purely an auditory nucleus. The tonotopic gradient of the ICC is arranged in such a way that low frequencies are represented in the dorsal 


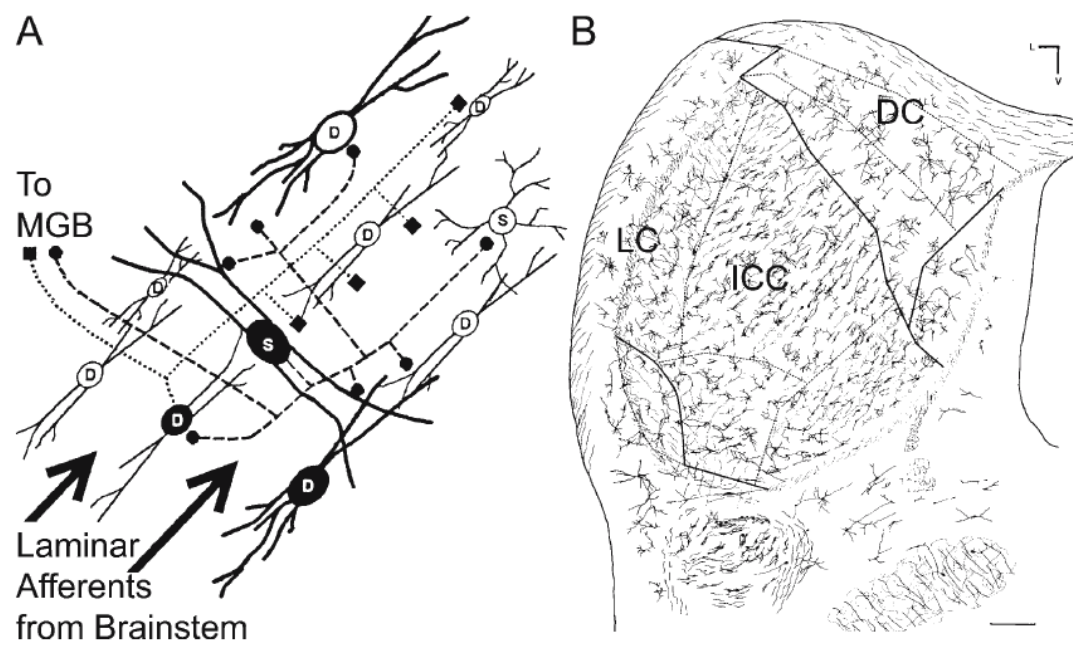

Figure 1.5: Neuronal organization of the Inferior colliculus. A, Representation of the cellular organization of the frequency lamina in the ICC. Disc-shaped neurons form the frequency lamina (D) while stellate neurons (S) connect different frequency laminas. B, Scheme of a coronal section of the IC that shows its different divisions. DC, dorsal cortex; LC, lateral cortex; ICC, central nucleus (scheme taken from Oliver 2005).

part and high frequencies are represented in more ventral areas (Figure 1.6). Neurons that respond to the same range of frequencies are grouped in rows and their dendritic fields are aligned in a narrow parallel way giving raise to frequency laminas (Oliver 2005). The result is that frequency representation in the IC is organized in discrete anatomical bands that extend along the rostrocaudal and medio-lateral axes (Stiebler \& Ehret 1985) (Figure 1.5A). This type of neuronal organization is well conserved and has been described in many species, from mice to humans (Casseday et al. 2005). Inputs from different auditory brainstem nuclei target particular locations of the frequency laminas generating discrete organized synaptic functional zones. For example, inputs from the dorsal cochlear nucleus arrive to the dorso-medial part of the lamina and inputs form the lateral superior olive arrive to the ventro-lateral part (Loftus et al. 2004; Loftus et al. 2010; Ono \& Ito 2015). It is believed that these functional zones are responsible for the different patterns of responses along the same frequency lamina (Lim \& Anderson 2007b; Straka et al. 2014). The ICC sends bilateral inhibitory and excitatory projections to the ventral and dorsal divisions of the medial geniculate body of the thalamus (MGBv) in a topographic order (Hackett et al. 2011; Ito et al. 2009; Ono \& Ito 2015; Mellott et al. 2014).

The lateral cortex or external nucleus (LC) is located lateral to the ICC. The main inputs to the LC come from the ipsilateral ICC, the auditory cortex, the spinal cord, and the dorsal column nuclei of the somatosensory nuclei suggesting a role in multisensory integration (Aitkin et al. 1978; Oliver 2005). The electrophysiological properties of neurons in the LC have been poorly studied compared to those of the ICC. They have broader tuning than the ICC, show less spontaneous 


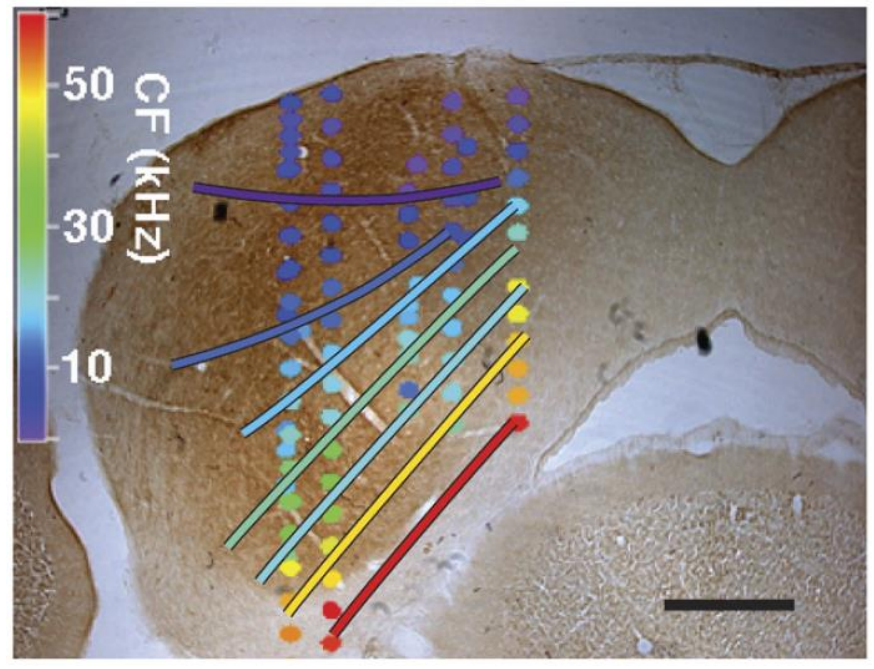

Figure 1.6: Frequency representation in the inferior colliculus of the mouse. Microphotograph of the inferior colliculus that shows the frequency distribution and orientation of the frequency lamina. Scale bar, $0.5 \mathrm{~mm}$ (figure taken from Portfors et al. 2011).

activity and respond to tactile stimulation (Aitkin et al. 1978; Syka et al. 2000). In mice, the low frequencies are best represented dorso-laterally and high frequencies ventro-medially (closer to the border of the ICC) (Stiebler \& Ehret 1985).

The dorsal cortex (DC) surrounds the IC from the dorsal side. In the cat the DC has a thickness around 200-300 $\mu \mathrm{m}$ in their most caudal part (Morest \& Oliver 1984). Although in cats the border between DC and ICC can be defined based on the sharp regression to low frequencies, indicating the beginning of the ICC (Merzenich \& Reid 1974), in mice and rats there is a continuous frequency representation that has complicated the electrophysiological delimitation of the border between these two nuclei (Stiebler \& Ehret 1985). Recently, using functional 2 photon imaging it has been shown that the DC in mice includes the first $100 \mu \mathrm{m}$ over the central part of the IC, and that DC neurons have wider receptive fields than neurons in the ICC (Barnstedt et al. 2015). The functional role of the $D C$ has not been systematically explored, but it is suggested that DC contributes to the integration of inter-collicular and descending information from the auditory cortex.

The IC is the auditory area that receives the largest density of corticofugal projections. It receives up to ten times more projections than the cochlear nucleus (Doucet et al. 2003). In the IC corticocollicular projections (CC) terminate mainly in the DC and LC and only weakly in the ICC (Figure 1.7). CC target the ICC in a tonotopic order while the projections that target DC and LC are more diffuse, suggesting two types of functional projections (Andersen et al. 1980; Winer et al. 1998; Lim \& Anderson 2007a; Markovitz et al. 2013; Straka et al. 2015). CC projections are excitatory, express Vglut1 and target mainly excitatory collicular cells (Ito \& Oliver 2010; Nakamoto et al. 2013; Ono \& Ito 2015). Electrophysiological experiments have shown that CC 

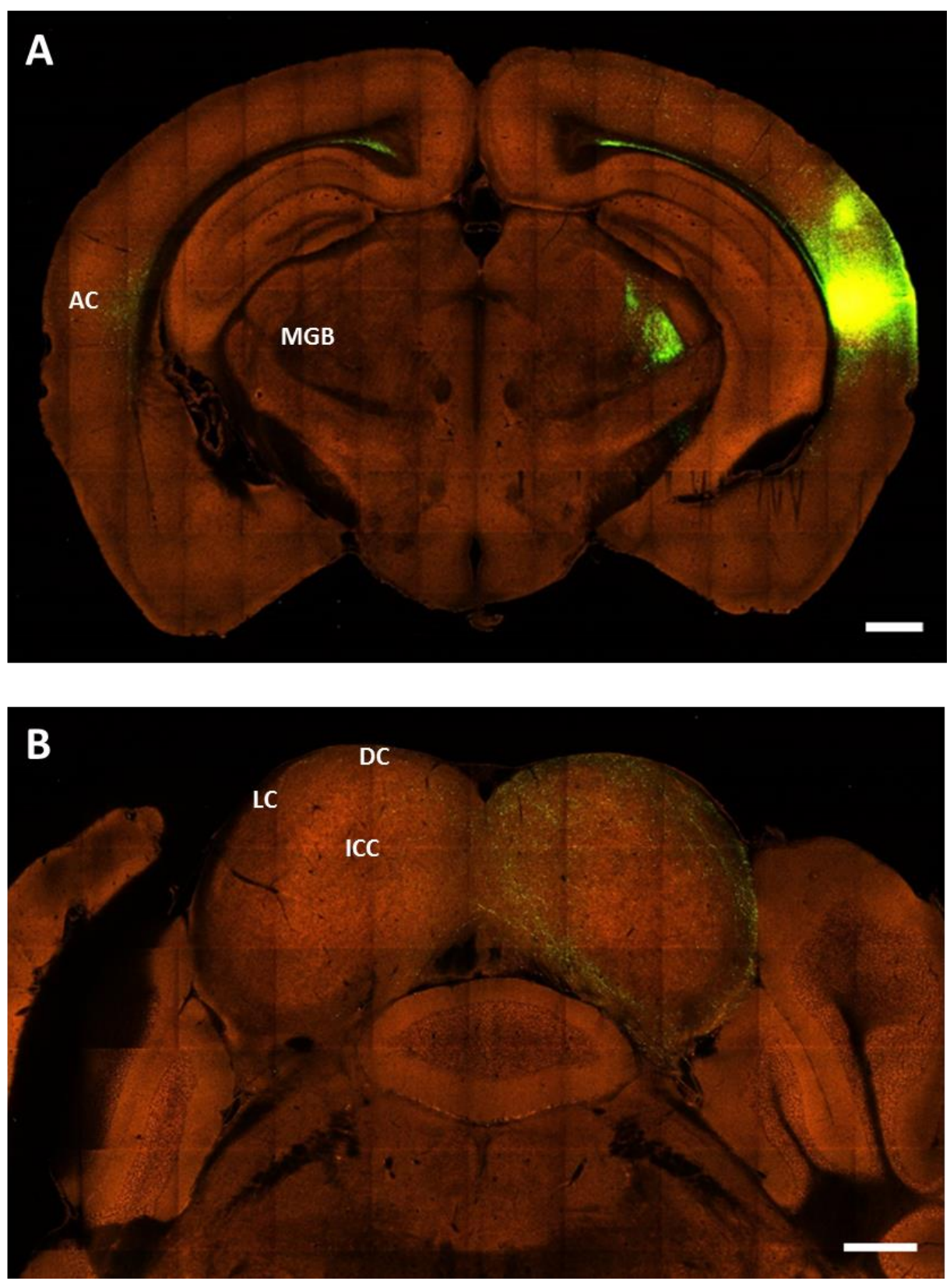

Figure 1.7: Cortico-collicular projections. A, Microphotograph of a mouse brain coronal section that shows the site of injection of AAV-GFP virus (green) in the auditory cortex (AC). Projections to the medial geniculate body (MGB) and the contralateral auditory cortex can be appreciated. B, Microphotograph of a coronal section of the same mouse in A that shows the inferior colliculus. Cortical projections (green) in the surroundings of the IC that correspond to the $L C$ and DC can be appreciated. Projections to the ICC are sparse. Scale bar $500 \mu \mathrm{m}$. Images modified from @2015 Allen Institute for Brain Science. Allen Mouse Brain Connectivity Atlas. http://connectivity.brain-map.org Experiment 112881858.

activation can lead to suppression of responses and spontaneous activity in the ICC, suggesting that CC activate also inhibitory neurons (Syka \& Popelár 1984; Bledsoe et al. 2003). CC projections can also lead to changes in the spectral tuning of collicular neurons in bats and mice (Suga et al. 2002; Yan \& Ehret 2001; Yan \& Ehret 2002; Wu \& Yan 2007). On the other hand, inactivation of the 
auditory cortex with TTX showed heterogeneous changes in firing rates of collicular neurons with no effect on thresholds and tuning properties (Popelář et al. 2003).

\subsubsection{Collicular experience-dependent plasticity by sound exposure during early postnatal development}

Compared to the auditory cortex, the effects of passive exposure during early development on the IC have received little attention. Collicular neurons of rats exposed during the first 4 months of life to sweep tones from 6 to $9 \mathrm{kHz}$ or 9 to $6 \mathrm{kHz}$ showed a preferential response to the exposed sweep tone (Clopton \& Winfield 1976). Tuning curves of IC neurons in mice reared from P8-P19 under constant exposure to trains of sharp sounds (clicks) or two-tones were broader (Sanes \& Constantine-Paton 1983; Sanes \& Constantine-Paton 1985). These pioneering studies showed that the collicular neurons are sensitive to the acoustic environment during early neuronal development. As in AC, exposure to pure tones, leads to an overrepresentation of the exposed tone in the tuning of IC neurons. Rats exposed to tones of 4 or $20 \mathrm{kHz}$ from P1-P21, and recorded under anesthesia 3 to 5 weeks after the end of the exposure, showed a higher number of collicular neurons tunes to the exposed tone than control animals (Poon \& Chen 1992). Using magneticresonance imaging, it has been shown that mice exposed from P9-P17 to 16 and $40 \mathrm{kHz}$, two frequencies that are typically represented in non-overlapping areas, and tested at P19, showed a larger responsive area that was responsive to both frequencies (Yu et al. 2007). In a more recent study, rats that were exposed to $14 \mathrm{kHz}$ from P9-P28 and tested at either P24 or P65 showed an expansion of the collicular responsive area tuned around $14 \mathrm{kHz}$ (Oliver et al. 2011). The collicular neurons of exposed animals had higher thresholds and larger responses at higher sound intensities than control neurons. Finally, a study where rats were exposed to $7.5 \mathrm{kHz}$ (from P9-P25) and tested right after the end of the exposure or 14 days later did not find changes in collicular frequency representation (Miyakawa et al. 2013). However, they found a transient narrowing of the bandwidth of neurons tuned around the exposed frequency that was no longer present 14 days after sound exposure. The effect of enriched acoustic environment (EAE) in the IC was recently evaluated (Bureš et al. 2014). The study used multiple complex sounds and one of them signaled the release of sweet syrup that was available for $2 \mathrm{~s}$. Rats were reared under this EAE conditions from P14-P28, and the responses of collicular neurons were recorded after 3-5 months. Collicular neurons showed a decrease in excitatory thresholds, a sharper tuning, and an increase in evoked and spontaneous activity. The same study evaluated the effects of this EAE conditions in adult animals. Rats were reared under EAE conditions at P90-P104 and tested immediately or one month later. In the collicular neurons recorded right after, EAE induced multiple changes: a decrease in excitatory thresholds, a sharper tuning and an increase in evoked and spontaneous activity. 
However, these effects were only seen in neurons with a BF higher than $8 \mathrm{kHz}$. Only the increased spontaneous activity and the sharper tuning persisted a month after EAE exposure ceased. Due to the complex nature of the stimulus, the effect its exposure had on frequency representation in this study was difficult to assess.

These results indicate that during early postnatal development, the IC can undergo persistent experience-dependent plasticity that might affect sound processing at the level of the IC, but also at downstream areas such as the AC.

\subsubsection{Collicular experience-dependent plasticity by associative learning during adulthood and the} role of the cortico-collicular projections

The study of the role of the CC and IC plasticity in classic conditioning was initiated by Nobuo Suga who used the bat as model. He found that, in collicular neurons of awake bats, a foot shock paired with tonal stimulation induced a decrease in the response to the original $\mathrm{BF}$ and an increase in the responses towards the frequency used for conditioning, generating a shift in BF. This change in tuning required cortical feedback. Inactivation of the auditory cortex with muscimol previous to conditioning prevents the shift in the collicular neurons. Interestingly, preventing the shift of collicular neurons with atropine application strongly reduces the shift in of cortical neurons (Ji et al. 2001). Compared to the shift in BF seen in AC the collicular shifts are short-term since they last around 2-3 h. Interestingly, shifts in BF only occur when the frequency of the conditioned tone was lower than the BF of the collicular neuron within $15 \mathrm{kHz}$ range and the average change was $1.1 \mathrm{kHz}$, suggesting that asymmetric connections between frequency laminas might exist in the IC (Gao \& Suga 1998; Gao \& Suga 2000; Ji \& Suga 2003). The collicular shifts in BF, in a similar way as the AC, are dependent on acetylcholine (Ji et al. 2001; Ma \& Suga 2003; Zhang et al. 2005) and collicular NMDA receptors (Ji et al. 2005) indicating that in the IC neuromodulatory centers can also play a permissive role in plasticity.

These studies have shown that the tuning properties of neurons in the IC of adult animals can also be modified due to associative training. More importantly, changes in the AC are dependent on plasticity at the level of the IC (a feedback loop that occurs through the CC), and highlights the need to integrate the IC into the equation of experience-dependent plasticity in the auditory system. Interestingly, studies on experience-dependent plasticity that used other models than bats and that explore other paradigms than classical conditioning have not been done.

\subsubsection{Plasticity in the auditory midbrain. Studies in humans}


The influence of experience in the processing of behaviorally relevant sounds such as speech has been successfully evaluated in humans using EEG recordings that reflect the auditory processing at the IC level. This technique is termed complex auditory brain stem responses (CABR) (Chandrasekaran \& Kraus 2010; Chandrasekaran et al. 2014; Kraus \& White-Schwoch 2015). It has been shown that the differences in CABR measures between adult native Mandarin Chinese and English speakers does not exist in neonates that will speak either of these two languages, suggesting that the differences observed in adults depend on the language exposure (Jeng et al. 2011). Musical training during childhood can enhance specific auditory features such as heightened high-frequency phase locking and response consistency (Skoe \& Kraus 2013; Wong et al. 2007). Finally, it has been shown also that speech processing is altered in disorders such as autism (Russo et al. 2008; Russo et al. 2009).

Since these studies have been performed in humans there are no specific neuronal correlates in the auditory pathway, particularly at the level of the IC, that account and explain the changes of previous experience seen in those studies.

The role of subcortical structures in experience-dependent plasticity has not been well studied. The inferior colliculus, given the features described above, is in a crucial cross-road between purely sensory information, modulatory, and cognitive inputs. For this reason this nucleus is the target of our study.

\subsection{Aim of the project}

The current doctoral project aims to understand how previous auditory experience will affect the way acoustic stimuli are sensed and where along the auditory pathway this interaction will start to affect the processing of auditory information. Based on the animal and human evidence summarized above and given its importance as a hub in the auditory pathway where multiple cortical and subcortical nuclei converge, I hypothesized that the IC is the site where previous auditory experience starts to influence the processing of incoming auditory information.

By using a combination of behavioral, electrophysiological and molecular tools I aimed to understand the following points:

The ability of auditory experience in the form of implicit auditory learning, during adulthood, to modify collicular frequency representations and sound processing;

The behavioral consequence of prior auditory experience on innate and learned frequency discrimination; 
The ability of frequency-discrimination learning to modify collicular frequency representations and sound processing, and the effect of previous auditory experience on this modification.

Addressing these points will contribute to better understanding of the influence of acoustic experience on the adaptive mechanisms of sound processing and the contribution of the IC to the filtering of auditory information. 


\section{MATERIALS AND METHODS}

\subsection{Animals}

Female mice C57BL/6JRj (Janvier labs, France) between 5 and 8 weeks old were used for all experiments. All animal experiments were approved and performed in accordance with the Niedersächsisches Landesamt für Verbraucherschutz und Lebensmittelsicherheit, project license number 33.14-42502-04-10/0288 and 33.19-42502-04-11/0658.

\subsection{Audiobox}

One day (exceptionally 2 days) after arrival, mice were anesthetized with an intraperitoneal (ip) injection of avertin (Tribromoethanol dissolved in Tert-amyl acohol; $1.5 \mathrm{~mL} / 100 \mathrm{grs}$ ) and a sterile transponder (ISO compliant 11784 transponder, $12 \mathrm{~mm}$ long, TSE, Germany) was implanted subcutaneously in the back. The small wound caused by the injection was closed with a drop of a topical skin adhesive (Histoacryl ${ }^{\circledR}$, Braun, USA). After one day (exceptionally 2-3) of recovery, animals were placed in the Audiobox.

The Audiobox (New Behaviour/TSE, Germany) is an automatic testing chamber that consists of two compartments connected by a corridor (Figure 2.1). Mice lived in groups of up to ten animals in the first compartment that consists of a normal mouse cage, where animals have ad libitum access to food. Water was available in the second compartment -the "corner"- that is located inside a sound attenuated chamber. Two ports are located in each side of the corner and are closed by sliding doors. To open the doors and gain access to the water, animals needed to nose-poked. Nose-pokes were detected by a sensor located in each port. An antenna located in the entrance of the corner identified the individual tag of the transponder. The individual visits to the corner were detected by coincident activity of a heat sensor and the reading of the transponder. The end of the visit was signaled when the heat sensor was no longer active and the antenna did not read any transponder. By detecting visits of specific animals, the Audiobox can select the stimulus to be presented accordingly and record the data of the activity occurred at single visits. A loudspeaker (22TAF/G, Seas Prestige) was located above the corner to present sound stimuli. The sounds presented were generated in Matlab (The MathWorks, USA) at a sampling rate of $48 \mathrm{kHz}$ and consisted of $30 \mathrm{~ms}$ pure tones with $5 \mathrm{~ms}$ slope, repeated at $3 \mathrm{~Hz}$ during the duration of the visits and a variable intensity of $70 \mathrm{~dB} \pm 5 \mathrm{~dB}$ at the center of the corner or the center of the homecage. The sound intensity was calibrated at the center of the corner with a Bruël \& Kjaer (4939 1/4" free field) microphone. To check for harmonic distortions, the microphone was placed at different positions within the corner, as well as outside the corner, sounds $(1-40 \mathrm{kHz})$ were played 


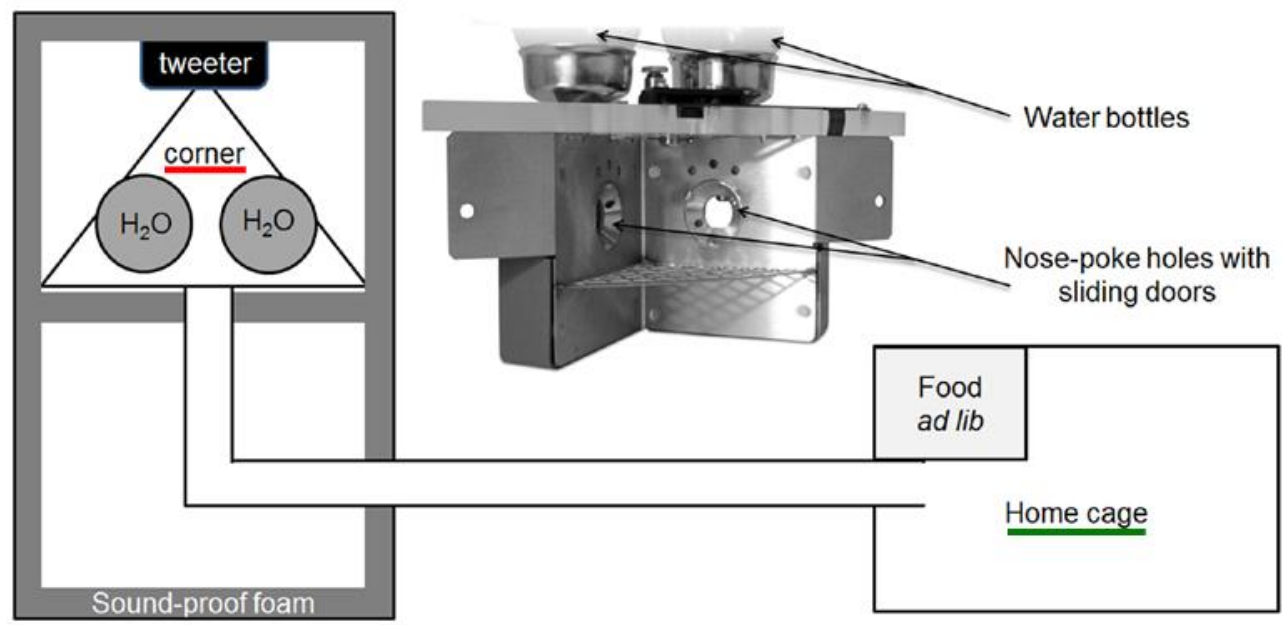

Figure 2.1: The Audiobox. Schematic representation of the Audiobox and a picture of the corner. Sound exposure took place in the corner (underscored in red) or the homecage (underscored in green) (modified from de Hoz \& Nelken, 2014).

at the 60-70 dB. Microphone signals were sampled at $96 \mathrm{kHz}$ and analyzed in Matlab. Tones between $3 \mathrm{kHz}$ and $19 \mathrm{kHz}$ did not show any significant harmonic distortion. The sounds presented inside the corner were attenuated outside the attenuated box ( $>20 \mathrm{~dB}$ ). However, there was little attenuation in the corridor located inside the attenuated box that was directly connected to the corner. Therefore, mice in the corridor inside the attenuated box could hear the sound played in the corner.

\subsection{Sound exposure}

All the experimental groups were first habituated to the Audiobox for three days. During the habituation phase, there was no sound presentation during the visits and the sliding doors in the corner remained open all the time, therefore nose-pokes were not required to access water.

After the habituation phase, a group of animals (exposed group) was exposed to a fixed tone pip of a specific frequency during every visit, regardless of nose-poke activity and water intake. The tone pips lasted the total duration of the visit. Another group of animals (random group) was exposed to a fixed tone pip in the homecage at random intervals. The sound was delivered by a loudspeaker (22TAF/G, Seas Prestige) located above the homecage and calibrated such that sound intensity was comparable to that inside the corner. The presentation of the sound was triggered by corner visits of a mouse living in another Audiobox. This ensured that the pattern and duration of sound presentation in the homecage was comparable to that experienced by each 


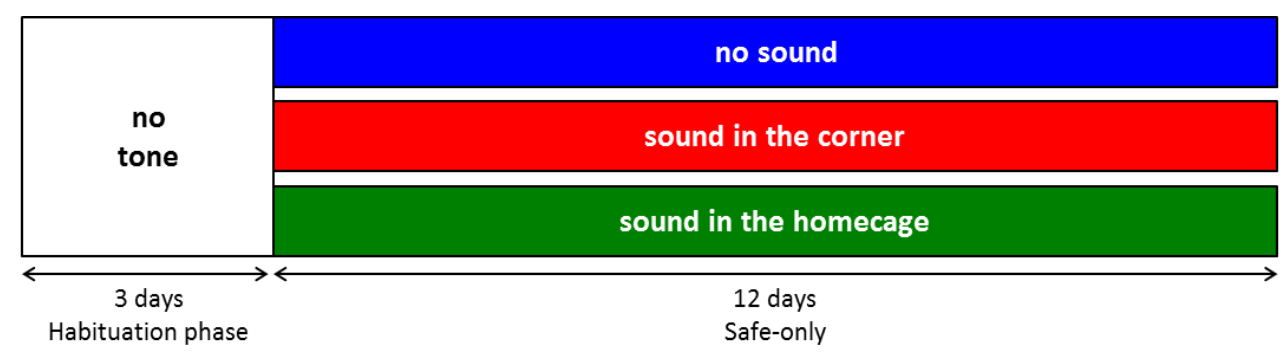

Figure 2.2: Sound exposure. Scheme of the sound exposure protocols, for the control (blue), exposed (red) and random (green) groups. The length of the boxes represent the time in the Audiobox.

mouse in the exposed group. This design prevented that the sound was associated with an action the mouse might make (such as a visit in the expose group) or an outcome (such as water in the exposed group), while ensuring a similar pattern of sound exposure as the exposed group. The sounds used during the exposure phase were: 8 or $16 \mathrm{kHz}$, depending on the experiment. The control group consisted of age matched animals that lived during the same amount of time in the Audiobox, without sound presentation (Figure 2.2).

\subsection{Electrophysiology}

\subsubsection{Surgery}

Mice were anesthetized with avertin for all experiments involving neuronal recordings (induction with $1.6 \mathrm{~mL} / 100 \mathrm{grs}$ and $0.16 \mathrm{~mL} / 100$ grs ip to maintain the level of anesthesia as needed). Anesthetized mice were placed on a stereotaxic apparatus (Kopf Inc., Germany) and the temperature of the animal was monitored by a rectal probed and maintained constant at $36^{\circ} \mathrm{C}$ (ATC 1000, WPI, Germany). The scalp was removed to expose the skull, and Bregma and Lambda were aligned to a plane level $\pm 50 \mu \mathrm{m}$. A metal head-holder was glued to the skull $1.3 \mathrm{~mm}$ rostral to Lambda. To access the left inferior colliculus (IC), a craniotomy of $2.8 \times 3 \mathrm{~mm}$ was made, with the center $1 \mathrm{~mm}$ lateral to the midline and $0.75 \mathrm{~mm}$ caudal to Lambda. The IC was identified by vascular landmarks (posterior to the transverse sinus anterior to the sigmoid sinus, Figure 2.3).

The tip of the left IC became visible after the craniotomy and measurements from the rostro-caudal and medio-lateral borders were made to place the recording electrode exactly in the middle of the IC, targeting the ICC. Extracellular multiunit recordings were made using mainly multi-electrode silicon arrays ( 1 shank, $177 \mu \mathrm{m}^{2}, 50 \mu \mathrm{m}$ electrode spacing, or 4 shanks, $177 \mu \mathrm{m}^{2}, 50 \mu \mathrm{m}$ electrode spacing, $150 \mu \mathrm{m}$ inter-shank spacing, Neuronexus Technologies, USA) (Figure 2.4) but also, occasionally, one glass-coated single electrodes: either glass coated tungsten electrodes, 


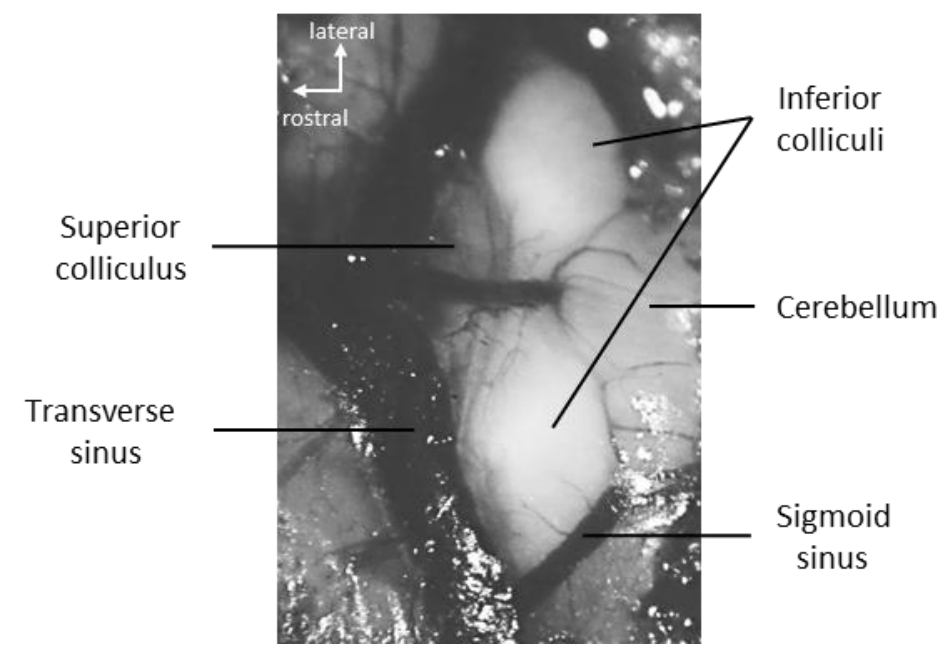

Figure 2.3: Location of the inferior colliculus. Photograph that shows a typical example of the brain area exposed after craniotomy. Both inferior colliculi can be appreciated. The borders of the IC were partially delimited by the transverse and sigmoid sinuses.

with a typical impedance of $900 \mathrm{mOhm}$, and an external diameter of 140 microns (AlphaOmega, Germany ); or glass coated platinum/tungsten electrodes, with a typical impedance of $1 \mathrm{mOhm}$ (ThomasRecordings, Germany). The electrodes were inserted in the central part orthogonally to the dorsal surface of the IC and lowered with a micromanipulator (Kopf Inc., Germany). In the case of single electrodes, recordings were made every 50-100 $\mu \mathrm{m}$. When multi-electrode silicon arrays were used, they were lowered (at a rate of $100 \mathrm{um} / 5 \mathrm{~min}$ ) until the upper electrode was in contact with the IC surface, visualized with a microscope (750 $\mu \mathrm{m}$ depth). The electrodes were labeled with Dil (1,1'-dioactedecyl-3,3,3,3'-tethramethyl indocarbocyanide, Invitrogen, Germany) to allow the reconstruction of the electrode track in postmortem sections (Figure 2.5).

The electric signal was amplified (HS-36 or HS-18, Neuralynx, USA) and sent to acquisition board (Digital Lynx 4SX, Neuralynx, USA). The raw signal was acquired at $32 \mathrm{kHz}$ sampling rate, bandpass filtered $(0.1-9000 \mathrm{~Hz})$ and stored for offline analysis. Recording and visualization was made by Cheetah Data Acquisition System (Neuralynx, USA). 
A

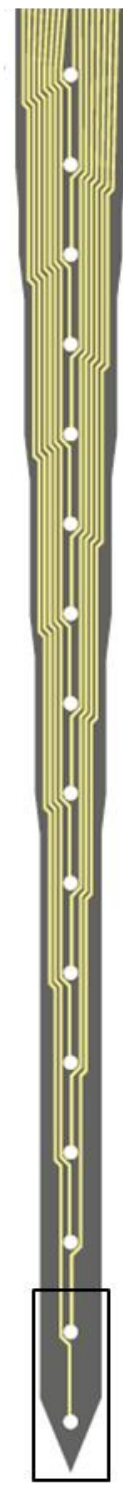

B
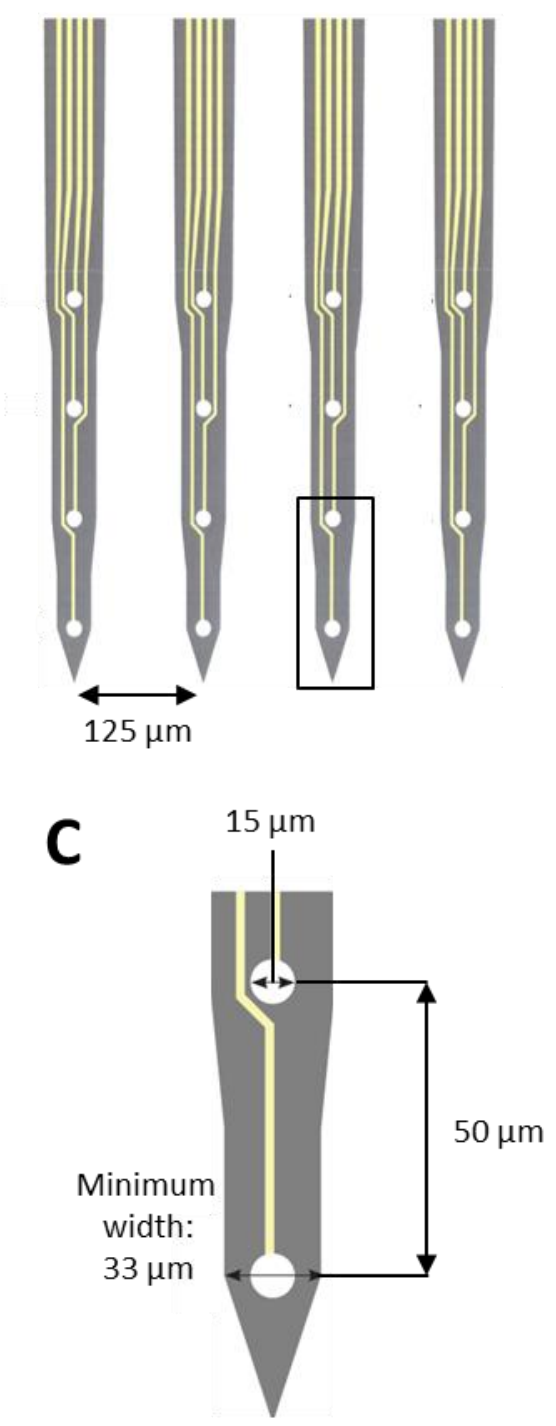

Figure 2.4: Multi-electrode arrays. Schematic representations of the multi-electrode arrays used. A, One shank with 16 electrodes (1x16). B, Four shanks with four electrodes in each (4x4). C, Close up showing the inter-electrode distance and the dimensions of the electrode (modified from Neuronexus catalog).

\subsubsection{Acoustic stimulation during electrophysiological recordings}

The experiments were performed in an acoustically isolated room. Sound stimuli consisted of 30 ms pure tone pips with $5 \mathrm{~ms}$ rise/fall slope of 24 frequencies $(3.3-24.6 \mathrm{kHz}, 0.125$ octave spacing) at different intensities (0-80 dB with steps of 5 or $10 \mathrm{~dB}$ ) played in a pseudorandom order every 500 ms. Each sound combination was played 5 times. The sound was synthesized using Matlab, produced by an USB interphase (Octa capture, Roland, USA), amplified (Portable Ultrasonic Power Amplifier, Avisoft Germany) and played in a free-field ultrasonic speaker (Ultrasonic Dynamic Speaker Vifa, Avisoft, Germany) located $15 \mathrm{~cm}$ horizontal to the right ear. The sound intensity was 


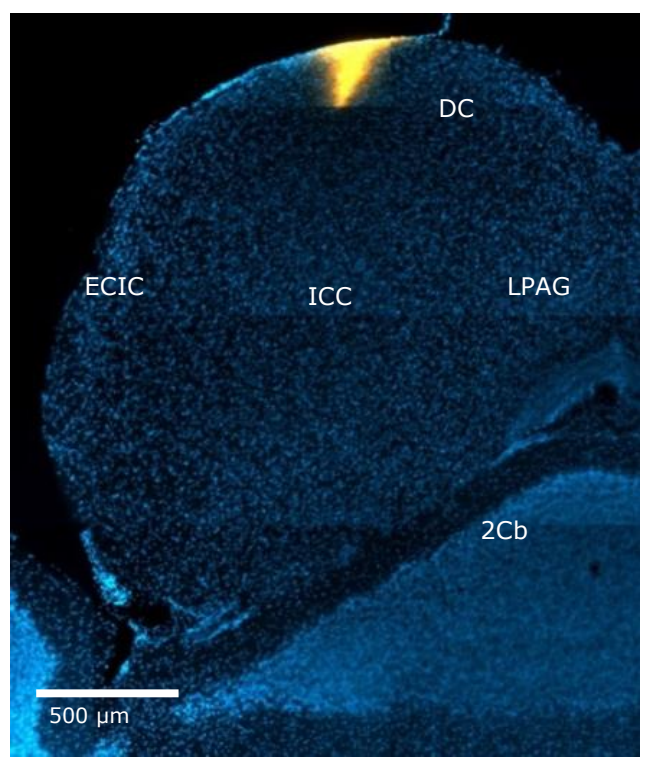

Figure 2.5: Location of the recording electrode. Photomicrograph of the IC showing the location of recording electrode dyed with Dil, section counterstained with DAPI. ECIC, external cortex of the IC; DC, dorsal cortex of the IC; ICC, central nucleus of the IC; LPAG, periaqueductal gray matter; $2 \mathrm{Cb}$, second cerebellar peduncle.

calibrated at the position of the animal's right ear with a Bruël \& Kjaer (4939 1/4" free field) microphone. Microphone signals were sampled at $96 \mathrm{kHz}$ and analyzed in Matlab. Tones between $2 \mathrm{kHz}$ and $30 \mathrm{kHz}$ did not show any significant harmonic distortion.

\subsubsection{Simultaneous cortical inactivation and collicular recording}

To study the corticofugal effect on collicular plasticity, the auditory cortex was inactivated with muscimol similar to Wu \& Yan, 2007. After the surgery in the IC, a $4 \times 3 \mathrm{~mm}$ craniotomy medial to squamosal suture and rostral of the lamboid suture was made to expose the left auditory cortex (AC). The AC was located dorsal and posterior of the transverse sinus (Guo et al., 2012). A small amount of Vaseline was applied to the boundaries of the craniotomy to form a well. A single electrode or a 16-channel multi-electrode array was inserted. Evoked responses to the tone pips were constantly monitored. A small amount of volume of phosphate buffered saline solution (Sigma, USA) was applied (3-5 $\mu \mathrm{L}$ ) every 10-15 min until obtain control recordings in the IC. After, 3-5 $\mu \mathrm{L}$ of muscimol were applied in the $A C(1 \mathrm{mg} / \mathrm{mL}$, dissolved in phosphate buffered saline solution, Sigma, USA) and evoked activity was monitored by playing frequency sweeps at $70 \mathrm{~dB}$

SPL or broad-band noise of different intensities every 5 minutes. AC was usually inactivated 15 minutes after muscimol application. Once cortical inactivation was confirmed, recordings in the IC were made again. 


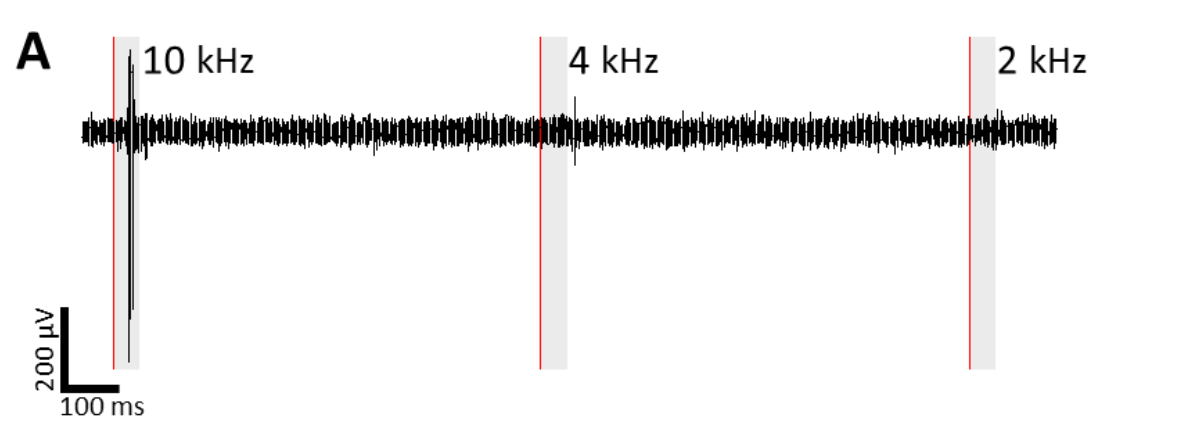

B

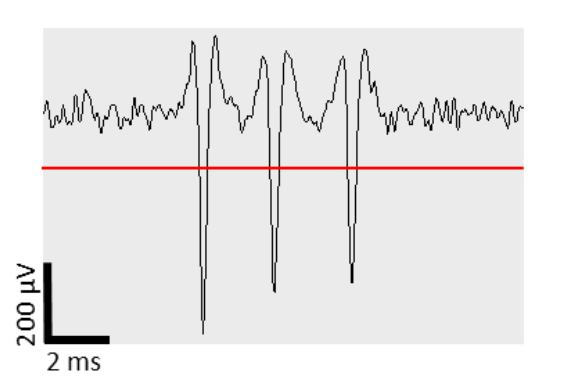

C

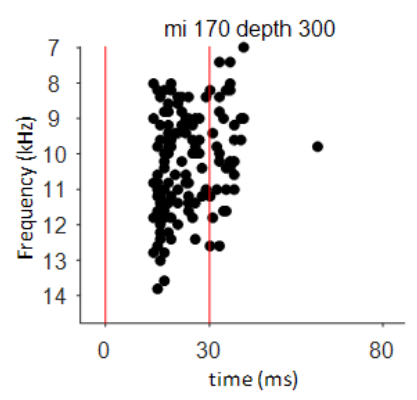

Figure 2.6: Electrophysiology. A, Filtered trace showing the responses to $10 \mathrm{kHz}$ at $70 \mathrm{~dB}$. B, Magnification of the response at $10 \mathrm{kHz}$ showing the shape of the evoked spikes, the horizontal red line indicates the threshold. C, Representative raster plot built from the detected spikes, vertical red lines indicate onset and offset of the sound.

\subsubsection{Analysis of electrophysiological recordings}

Offline analysis was performed in custom written scripts in Matlab. The stored signals were highpass filtered $(450 \mathrm{~Hz})$. To improve the signal-to-noise ratio in the recordings with the silicon probes, the common average reference (CAR) was calculated by averaging all the functional channels and subtracted from each channel, as described in (Ludwig et al. 2009). Multiunit spikes were then detected by setting a threshold that was 6 times the median absolute deviation of each channel (Figure 2.6).

To determine the presence of auditory responses, first a peri-stimulus time histogram (PSTH) was built, with $1 \mathrm{~ms}$ bin size, combining all the frequencies and the intensities above $30 \mathrm{~dB}$. The overall spike counts of the 80 ms windows before and after tone onset were compared $(p<0.05$, unpaired t-test). To characterize only the excitatory responses and to avoid mixing them with suppressive responses (when sound inhibits spontaneous activity, $<10 \%$ of cases), a positive threshold of the difference of spikes before and after onset was set. This threshold was empirically determined by visualizing and counting the minimum spike number required to observe an excitatory response. 
Finally, to classify a recorded site as sound driven two criteria were set: a significant difference in the PSTH between intervals of $80 \mathrm{~ms}$ before and after sound onset, and a positive spike count of at least 45 spikes. With these criteria $85 \%$ of the recorded sites where classified as sound driven.

With the sound driven sites, the spikes across all the trials for each frequency-intensity combination were summed. Then, the number of spikes in an interval of $80 \mathrm{~ms}$ from tone onset was calculated and the spontaneous activity removed (calculated as the number of spikes $80 \mathrm{~ms}$ before tone onset). This yielded a specific spike count per each frequency-intensity combination that was used to build frequency response areas (FRA), as well as tuning curves at different sound intensities. FRA were generated by color-plotting the sum of spikes, as described above, for each frequenciesintensity combination (Figure 2.7).

To calculate the best frequency ( $B F$, frequency that elicited the best response in that population of cells), first, the FRA was smoothed by a $3 \times 3$ gaussian median filter (Guo et al. 2012; Polley et al. 2013). The smoothed spike counts were summed over all intensities and the frequency with the highest spike count was selected as the BF. In the rare cases where more than one frequency elicited the highest response, the mean was used as BF.

The threshold, the lowest sound intensity that elicited a reliable response, the calculated from smoothed FRA as the lowest sound intensity that elicited a spike count 1.5 times higher than the spontaneous activity (Schreiner \& Sutter 1992).

Temporal response areas for a given frequency were calculated from the PSTHs evoked by that frequency simultaneously at different IC depths. The PSTH was calculated from the sum of spikes over all intensities and all trials in $5 \mathrm{~ms}$ bins. The counts across time were represented against depths of recording (i.e. Figure 3.8).

\subsection{Gene expression analysis}

To investigate whether sound exposure induced changes in collicular or cortical expression of genes related with neuronal plasticity, quantitative real time PCRs (qPCR) were made. The genes were chosen (Table 2.1) based on previous reports that showed changes in expression upon sound exposure, acoustic learning paradigms or enriched environment (Ortinski et al. 2004; Dong et al. 2010; Holt et al. 2005; Browne et al. 2012; Marianowski et al. 2000; Tan et al. 2007; Mainardi et al. 2010). The analysis involved several steps: i) tissue collection; ii) RNA extraction; iii) cDNA synthesis; iv) quantitative PCR. 

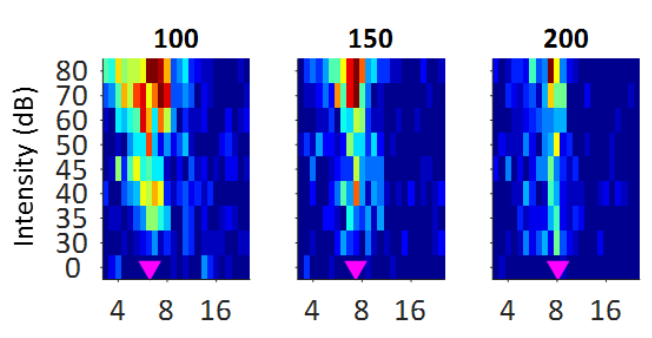

Depth $(\mu \mathrm{m})$

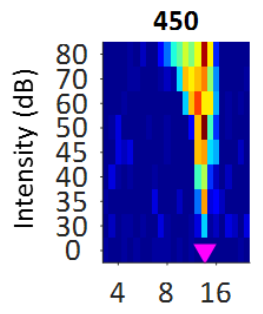

$4 \quad 8 \quad 16$

$4 \quad 8 \quad 16$

500
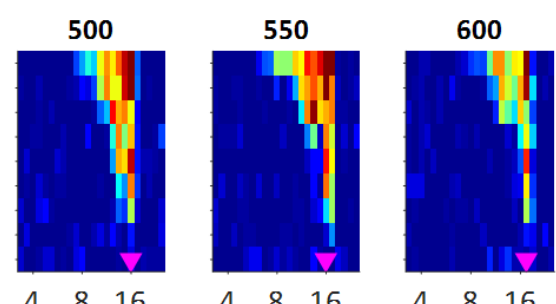

$\begin{array}{lll}4 & 8 & 16\end{array}$
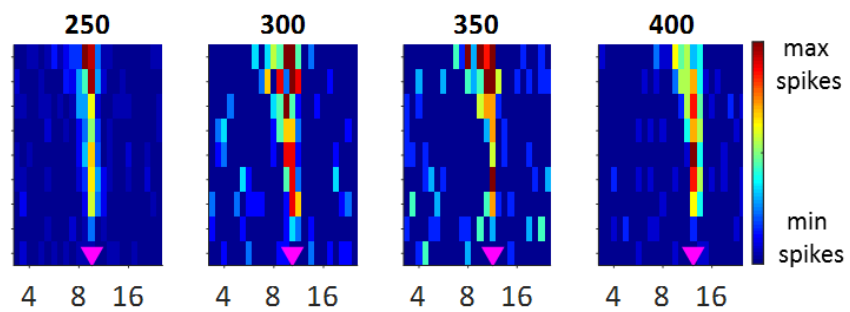

$\begin{array}{lll}4 & 8 & 16\end{array}$

$4 \quad 8 \quad 16$

650

700

750

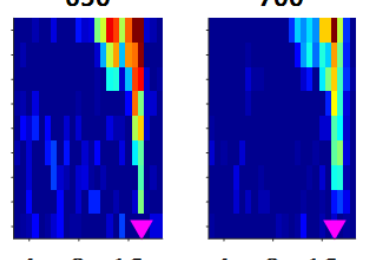

$4 \quad 816$

$4 \quad 8 \quad 16$

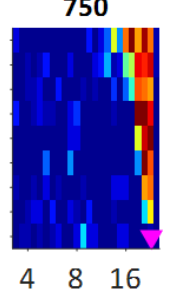

Frequency $(\mathrm{kHz})$

Figure 2.7: Frequency response areas in the IC. Representative example of frequency response areas recorded simultaneously in the central part of the IC at different depths. The magenta arrows indicate the best frequency for each depth.

\subsubsection{Tissue collection}

Mice used for gene expression analysis were not those used for IC recordings, but were treated the same way. After 3 days of habituation and 7 days of sound exposure in the Audiobox, mice were anesthetized with avertin and killed by cervical dislocation, immediately the brain was extracted and both inferior colliculi and in some cases the AC were dissected and immediately frozen at -80 ${ }^{\circ} \mathrm{C}$ and stored for later analysis.

\subsubsection{RNA extraction}

RNA was extracted from the dissected samples using a combination of Qiazol Reagent and RNeasy kit, each according to manufacturer's instructions (Qlagen,Germany). To the frozen samples, $1 \mathrm{ml}$ of Qiazol was added and homogenized immediately for 1 min using a rotor stator ultramax and incubated for $5 \mathrm{~min}$ at room temperature. Then, the samples were centrifuged at 14,000 rpm for $15 \mathrm{~min}$ at $4^{\circ} \mathrm{C}$. The aquatic phase was transferred into a new tube ( $\left.600 \mu \mathrm{l}\right)$ and the same volume of ethanol $70 \%$ RNA-grade was added and mixed vigorously for $15 \mathrm{~s}$. Next, the sample was loaded onto an RNeasy Mini Spin Column in a $2 \mathrm{ml}$ collection tube and centrifuged $1 \mathrm{~min}$ at 12,000 rpm. $700 \mu \mathrm{l}$ of RW1 buffer were added followed by 1 min centrifugation at 12,000 rpm. The flow through was discarded and the collection tube was replaced. $500 \mu$ l of RPE buffer were added 
Table 2.1: Genes analyzed with qPCR.

\begin{tabular}{|c|c|c|}
\hline Category & Protein & Gene \\
\hline \multicolumn{3}{|c|}{ Transcription factors and immediate early genes } \\
\hline & Arc & $\operatorname{arc}$ \\
\hline & C-fos & $c f o s$ \\
\hline & cAMP response element-binding (CREB) & $c r e b$ \\
\hline \multicolumn{3}{|l|}{ Inhibitory transmission } \\
\hline & GABAa receptor: & \\
\hline & subunit $\beta 2$ & gabrb2 \\
\hline & subunit $\beta 3$ & gabrb3 \\
\hline & subunit $\alpha 1$ & gabra1 \\
\hline & subunit $\alpha 3$ & gabra3 \\
\hline & Glutamic acid descarboxylase & $\operatorname{gad} 1$ \\
\hline & GABA vesicular transporter (VGAT) & vgat \\
\hline \multicolumn{3}{|l|}{ Excitatory transmission } \\
\hline & NMDA receptor: & \\
\hline & subunit $2 \mathrm{~A}$ & $\operatorname{grin} 2 a$ \\
\hline & subunit 2B & $\operatorname{grin} 2 b$ \\
\hline & AMPA receptor: & \\
\hline & subunit 1 & gria1 \\
\hline & subunit 2 & gria2 \\
\hline & Post-synaptic density protein 95 (PSD95) & psd95 \\
\hline & Glutamate vesicular transporter 2 (Vglut2) & vglut2 \\
\hline \multicolumn{3}{|c|}{ Neurotrophic factors and neuronal plasticity } \\
\hline & Brain derived neurotrophic factor (BDNF) & $b d n f$ \\
\hline & Matrix-metalloprotease 9 (MMP9) & $m m p 9$ \\
\hline & Cholinergic receptor muscarinic 2 & chrm2 \\
\hline
\end{tabular}

followed by 1 min centrifugation at 12,000 rpm, this step was repeated once. The column was centrifuged during $2 \mathrm{~min}$ at 12,000 rpm with the lid open to dry the column from traces of ethanol. For the elution step the column was transfer to a new tube and $20 \mu$ l of RNA-grade water was loaded onto the column and centrifuged for $1 \mathrm{~min}$ at 12,000 rpm. The RNA concentration and quality was assessed in a nano spectrophotometer.

\subsection{3 cDNA synthesis}

The Superscript III kit to synthesized cDNA was used (Invitrogen Life Technologies, Germany). For each sample $1 \mu \mathrm{g}$ of RNA was transferred into a PCR tube. $1 \mu \mathrm{l}$ of dT-Mix primer $(0.6 \mathrm{pmol} / \mu \mathrm{l}$, ID 9578-4) and $1 \mu \mathrm{l}$ N9-random primer (120 pmol/ $\mu \mathrm{l}, \mathrm{ID} 4542)$ was added to the PCR tube, and incubated for $2 \mathrm{~min}$ at $70^{\circ} \mathrm{C}$. The tubes were placed on ice right after. Then, $2.6 \mu \mathrm{l}$ of $5 \times 1^{\text {st }}$ strand buffer, $1 \mu \mathrm{l}$ of $0.1 \mathrm{M} \mathrm{DTT}, 2 \mu \mathrm{l}$ of a mix of deoxynucleotides (100 mM each), $1 \mu \mathrm{l}$ of Superscript III $(200 \mathrm{U} / \mu \mathrm{l})$ were added for a final volume of $13 \mu \mathrm{l}$. The mix was placed in the thermocycler to incubate $10 \mathrm{~min}$ at $25^{\circ} \mathrm{C}, 45 \mathrm{~min}$ at $50^{\circ} \mathrm{C}$, and $45 \mathrm{~min}$ at $55^{\circ} \mathrm{C}$. Then, the tubes were placed on ice and diluted with water to a final volume of $200 \mu \mathrm{l}$. 
Table 2.2: Primers used for qPCR.

\begin{tabular}{|c|c|c|}
\hline Gene & Forward 5'-3' sequence & Reverse 5'-3' sequence \\
\hline $\operatorname{Arc}$ & AGGGGCTGAGTCCTCACA & GACTTCTCAGCAGCCTTGAGAC \\
\hline$B d n f$ & GCATCTGTTGGGGAGACAAG & TGGTCATCACTCTTCTCACCTG \\
\hline Cfos & TCGACCTAGGGAGGACCTTACC & CCAGATGTGGATGCTTGCAA \\
\hline Chrm2 & AAAGGCTCCTCGCTCCAG & AGTCAAGTGGCCAAAGAAACA \\
\hline Creb & CCACTGATGGACAGCAGATTC & GGTATGTTTGTACATCGCCTGA \\
\hline Gabra1 & GCCCACTAAAATTCGGAAGC & CTTCTGCTACAACCACTGAACG \\
\hline Gabra3 & CTTGGGAAGGCAAGAAGGTA & TGGAGCTGCTGGTGTTTTCT \\
\hline Gabrb2 & GGGTCTCCTTTTGGATTAACTATGA & GGTCATTGTTAGGACAGTTGTAATTC \\
\hline Gabrb3 & CTCCATTGTAGAGCACCGTCT & TCAATGAAAGTCGAGGATAGGC \\
\hline Gad1 & TGGGATTTGAAAACCAGATCA & GAAACAGACATTTGTGTGCTCAG \\
\hline Gria1 & AGGGATCGACATCCAGAGAG & TGCACATTTCCTGTCAAACC \\
\hline Gria2 & CAAACACTGCAATTTTAGATCTCC & CCAGGCATCCTGTTCCAG \\
\hline Grin2a & CCTTGAGGTCAACAGCATCA & GCATAACATGCTCTGAAATATACACA \\
\hline Grin $2 b$ & GGGTTACAACCGGTGCCTA & CTTTGCCGATGGTGAAAGAT \\
\hline Mmp9 & CAGAGGTAACCCACGTCAGC & GGGATCCACCTTCTGAGACTT \\
\hline Psd95 & ACTCCTGCTCCAGCTTCGT & GGAGCTCCAGGGAGACAGT \\
\hline Rpl13a & ATCCCTCCACCCTATGACAA & GCCCCAGGTAAGCAAACTT \\
\hline Vgat17 & ACGTGACAAATGCCATTCAG & TGAGGAACAACCCCAGGTAG \\
\hline Vglut2 & GGAAAATCCCTCGGACAGA & TGGTCTCTCGGTTGTCCTG \\
\hline
\end{tabular}

\subsubsection{Primers}

The primers for most of the genes were available at the AGCT-Lab of the Max Planck Institute of Experimental Medicine, Göttingen, Germany, with the exception of the genes: gabra1, gabrb2 gabrb3, gria1, and grin2a that were designed using the Assay Design Center for Universal Probe Library by Roche (http://lifescience.roche.com), and synthesized in the AGCT-Lab. Primers are listed in the Table 2.2.

\subsubsection{Quantitative PCR}

For quantitative qPCR, SyBr Green Master Mix kit (Applied Biosystems, Germany) was used, and amplification reactions were run on a Roche LC480 Detection System (384 well plates) or 7500 Fast Real-Time PCR System (96 well plates). Reactions were run in four replicates. For each replicate, 2 $\mu \mathrm{l}$ of $\mathrm{cDNA}, 0.2 \mu \mathrm{l}$ of forward primer, $0.2 \mu \mathrm{l}$ of reverse primer, $5 \mu \mathrm{l}$ of SyBr Green Master Mix and 2.6 of water were added into each well. The amplification program was as follows: 2 min at $50^{\circ} \mathrm{C}$, $10 \mathrm{~min}$ at $95^{\circ} \mathrm{C}, 15 \mathrm{~s}$ at $95^{\circ} \mathrm{C}$ followed by $1 \mathrm{~min}$ at $60^{\circ} \mathrm{C}$, the last 2 steps were repeated 40 times. The melting curves were obtained using the following running program: $15 \mathrm{~s}$ at $95^{\circ} \mathrm{C}, 1 \min 60^{\circ} \mathrm{C}$ and $15 \mathrm{~s}$ at $95^{\circ} \mathrm{C}$. Ct values and melting curves were obtained using the software provided for each 
system. The efficiency $(E)$ of each pair of primers was estimated based on the slope $(m)$ of a standard curve of the $C t$ values from 5 serial logarithmic dilutions of a template cDNA, using the following formula:

$$
E=10^{\left(\frac{-1}{m}\right)}
$$

The goodness of fit $\left(R^{2}\right)$ of all the standard curves was $>0.98$.

Gene expression relative to the housekeeping gene (Rpl13a) was calculated with the method used by (Smith et al. 2014), where corrections for different efficiencies between target gene and housekeeping gene are made:

$$
R E=\frac{E k h g^{C T h k g}}{E t g^{C T t g}}
$$

Where $R E$ is the relative expression, Ekhg is the efficiency of the housekeeping gene, CThkg is the $C t$ value of the housekeeping gene, Etg is the efficiency of the target gene, and CTtg is the Ct value of the target gene.

\subsection{Immunolabeling for presynaptic markers}

To investigate whether sound exposure induces changes in the expression of presynaptic proteins in the IC, different immunolabelings were performed by Olga Babaev (Neurobiology department, Max Planck Institute of Experimental Medicine, Göttingen). Prior to immune-histochemical procedure, mice were paired according to day of sound exposure. Brains were dissected and post fixed in PFA 4\% over night, and cryoprotected in 30\% sucrose in 0.1 PB. Free floating coronal sections of 40 micrometers thickness taken from rostral to caudal parts of inferior colliculus (from -0.55 to $-0.95 \mathrm{~mm}$ from lambda) were prepared using a Leica CM3050S cryostat (Leica, Wetzlar, Germany). The sections were incubated in blocking solution ( $10 \%$ bovine serum albumin, $3 \%$ goat serum, $0.3 \%$ Triton-X in $0.1 \mathrm{M}$ phosphate- buffered saline (PBS)) for 1 hour. Primary antibody for Vglut2 (guinea pig polyclonal antibody, clone 135 404, Synaptic Systems, Göttingen, Germany) was diluted to 1:2000 and primary antibody for Vgat (rabbit polyclonal antibody, clone 131 002, Synaptic Systems, Goettingen, Germany) was diluted to 1:500, both in blocking solution. Sections were incubated with both antibodies simultaneously for 24 hours. Secondary antibodies, goat anti guinea- pig polyclonal antibody conjugated to Alexa Fluor 555 and goat anti rabbit polyclonal antibody conjugated to Alexa Fluor 488 (Invitrogen, Darmstadt, Germany) were diluted to 1:600 in blocking solution and applied to sections for 2 hours. The sections were washed with PBS after each 
incubation step, and were finally mounted on glass slides using Aqua-Poly/ Mount (Polysciences, Eppelheim, Germany).

\subsubsection{Imaging of synaptic markers}

Images of synaptic markers Vglut2 and VGAT were obtained using a confocal laser scanning microscope (Leica SP2; Leica, Wetzlar, Germany) and 63x oil immersion objective. For each controlexposed pair, sections were anatomically matched and the settings for laser power, gain and offset were kept constant during imaging. After scanning through $\mathrm{Z}$ axis of each section to determine the area with highest penetration of both antibodies (defined as an area with highest fluorescent intensity), one stack of two optical sections with parameters $238 \mu \mathrm{m} \times 238 \mu \mathrm{m} \times 2 \mu \mathrm{m}$ (height, length, width) was obtained separately from dorsal (300 $\mu \mathrm{m}$ depth) and ventral areas (600 $\mu \mathrm{m}$ depth). In total, 10 stacks of each area were obtained per animal.

\subsubsection{Image analysis}

Quantification of Vglut2 and VGAT positive puncta was performed with IMARIS software (Bitplane, Switzerland) using "spots" function. Threshold to eliminate background staining was applied using automatic threshold function in IMARIS and positive puncta were defined as spots with $1 \mu \mathrm{m}$ diameter above threshold intensity value. Total number of Vglut2 and VGAT positive puncta was quantified for dorsal and ventral areas in each animal and the ratio between total number of VGAT and the total number of Vglut2 was calculated in EXCEL.

\subsection{Single unit recording from cochlear nucleus}

To investigate whether sound exposure induces plastic changes in the cochlear nucleus, recordings in the cochlear nucleus were performed by Dr. Zhizi Jing and Dr. Nikola Strenzke (Auditory Systems Physiology Group, InnerEarLab, University Medical Center, Göttingen). Mice were placed in the Audiobox as usual. The control group heard no sound upon entering the corner and the exposed groups heard an $8 \mathrm{kHz}$ tone. Six to 12 days after beginning of sound exposure mice were removed from the Audiobox one a time for acute electrophysiology. Mice were anesthetized with urethane $\left(1.32 \mathrm{mg} / \mathrm{kg}\right.$, ip) and xylazine ( $5 \mathrm{mg} / \mathrm{kg}$, ip). Animal temperature was maintained at $36.5^{\circ} \mathrm{C}$ using a custom-designed heating pad in a sound proof chamber with ambient temperature of $33{ }^{\circ} \mathrm{C}$. A tracheotomy was performed and the cartilaginous ear canals were removed before positioned in a custom-designed head-holder and stereotaxic apparatus. Then a craniotomy was performed on part of the occipital bone. The surface of cochlear nucleus was exposed by removing part of the cerebellum. Glass microelectrode filled with $2 \mathrm{M} \mathrm{NaCl}$ and $1 \%$ methylene blue was advanced in 4 um steps (Inchworm micromanipulator, EXFO Burleigh, Germany) through the cochlear nucleus. 
Once single unit was isolated, the spontaneous rate, characteristic frequency (CF) and best threshold for each unit was determined. 200 repetitions of $50 \mathrm{~ms}$ tone burst at CF $\left(2.5 \mathrm{~ms} \cos ^{2}\right.$ rise/fall, $10 \mathrm{~Hz}$ repetition rate) and $8 \mathrm{kHz}$ were used to characterize sound evoked responses. To map the receptive area of each unit, frequency sweeps of $30 \mathrm{~ms}$ tones at $70 \mathrm{~dB}$ (10 repetitions per sweep, $3 \mathrm{~Hz}$ repetition rate) of frequencies ranging from $4 \mathrm{kHz}$ to $30 \mathrm{kHz}$ (13 frequencies in total) were presented. Extracellular signals were amplified, bandpass filtered (300-3000 Hz) using ELC03X amplifier (NPI Electronic, Tamm, Germany). Digitized signals (TDT system 3) were saved for offline analysis using custom written Matlab software. Unit classification was based on their response characteristics to supra-threshold ( $30 \mathrm{~dB}$ above threshold) $50 \mathrm{~ms}$ tone bursts presented at the characteristic frequency (Pfeiffer, 1966).

\subsection{Behavioral experiments}

\subsubsection{Pre-pulse inhibition}

To evaluate whether sound exposure in the corner or the homecage affected frequency discrimination acuity, pre-pulse inhibition (PPI) of the acoustic startle response (ASR) was used (Clause et al. 2011; Aizenberg \& Geffen 2013a; Aizenberg et al. 2015). The acoustic startle response is a reflexive motor response due to an unexpected loud noise that can be attenuated by the presentation of a weak sound right before the loud noise (Koch 1999).

The experiments were performed in an acoustically isolated room kept in darkness. Animals were placed in a custom-made acrylic chamber of $12 \times 4 \mathrm{~cm}$. Movements of the animal were detected by a piezoelectric sensor located below the chamber. The signal was sent to an acquisition board (National Instruments, USA), digitized (1 kHz sampling rate) and sent to the computer for storage and analysis. Custom written routines in Matlab were used for visualization and analysis of the data. The sound was synthesized using Matlab, produced by an USB interphase (Octa capture, Roland, USA), amplified (Portable Ultrasonic Power Amplifier, Avisoft Germany) and played in a free-field ultrasonic speaker (Ultrasonic Dynamic Speaker Vifa, Avisoft, Germany) located $15 \mathrm{~cm}$ away from the chamber on the horizontal plane.

The trials were divided in "startle-only trials" and "pre-pulse trials" and consisted of a frequency change from $1 \mathrm{~ms}$ ramp from the background tone $(\mathrm{f} 1)$ to the pre-pulse tone $(\mathrm{f} 2)$ at constant $70 \mathrm{~dB}$ SPL (Figure 2.8). The frequency change remained constant for $80 \mathrm{~ms}$ and immediately followed by $20 \mathrm{~ms}$ of broad-band noise (BBN) at $\sim 100 \mathrm{~dB}$. The background tone was presented again right after the $\mathrm{BBN}$ and kept constant until the following trial. For the startle-only trials $\mathrm{f} 1$ and $\mathrm{f} 2$ were $16 \mathrm{kHz}$ and for pre-pulse trials, f2 were 15.92, 15.84, 15.68, 15.472, 15.2, 14.72, 14, and $8 \mathrm{kHz}$ that 
corresponded to a $\Delta f$ of: $0.5,1,2,3.3,5,8,12.5$ and $50 \%$, respectively. All the trials were presented randomly between 8 and $25 \mathrm{~s}$. To avoid silent gaps between trials, a sound file that included all the sound phases was generated, saved in wav format and played at once.

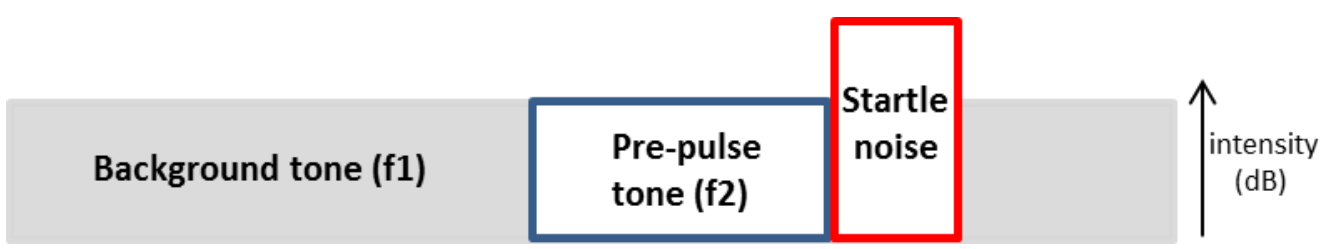

Figure 2.8: Pre-pulse inhibition of the acoustic startle response. PPI consisted of three different sounds, a background tone ( $\mathrm{f} 1$ ), a pre-pulse tone ( $\mathrm{f} 2$ ) and a startle noise. $\mathrm{f} 1$ and $\mathrm{f} 2 \mathrm{had}$ an intensity of $70 \mathrm{~dB}$ while the startle noise was $100 \mathrm{~dB}$.

The experiment was divided in 5 phases followed one after the other uninterruptedly. 1) Chamber habituation: at the start of each session, animals were placed in the test chamber and allowed to habituation for 10 minutes; 2) Sound habituation: a constant background tone ( $\mathrm{f} 1: 16 \mathrm{kHz}, 70 \mathrm{~dB}$ SPL) was played for 5 minutes; 3 ) Startle-only trials: 10 startle-only trials were presented on the background of $16 \mathrm{kHz}$ to allow for short-term habituation to the startle sound; 4) Test phase: 10 pre-pulse trials and 10 startle only trials were presented to assess frequency discrimination; 5) Startle-only trials: 5 startle-only trials were presented to check for habituation over the duration experiment.

The ASR was measured as the maximal vertical force that occurred in a $200 \mathrm{~ms}$ window starting with the onset of the startle noise, minus the mean of the force of $50 \mathrm{~ms}$ before the startle noise. For each animal, the startle-only trials of the test phase and the pre-pulse trials of each frequency were averaged (Figure 2.9A,B). The percent of inhibition for each pre-pulse frequency PPI(\%) was calculated as follows:

$$
\operatorname{PPI}(\%)=100 * \frac{\text { ASRnopps }- \text { ASRpps }}{\text { ASRnopps }}
$$

Where, ASRnopps is the mean response of the startle-only trials and ASRpps is the mean response of the pre-pulse trials for that particular frequency.

Discrimination thresholds (Th) for each animal defined as the $\Delta f$ that caused $50 \%$ of inhibition of the maximum response were calculated from parametric fit to a generalized logistic function (fit function Matlab) (Aizenberg \& Geffen 2013a) (Figure 2.9C). 


$$
P P I=-\frac{a}{2}+\frac{a}{1+\exp (b+c \Delta f)}
$$

Animals with a fit coefficient of the curve $\left(R^{2}\right)$ below 0.7 were excluded from statistical analysis. Additionally, the pooled data for each group was also fitted to a generalized logistic function.

A

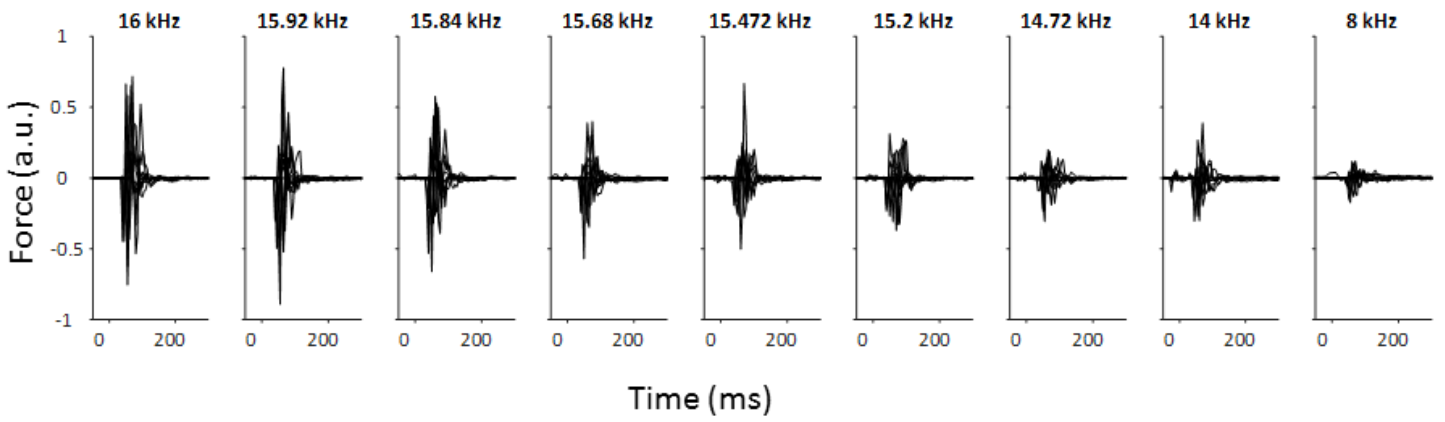

B

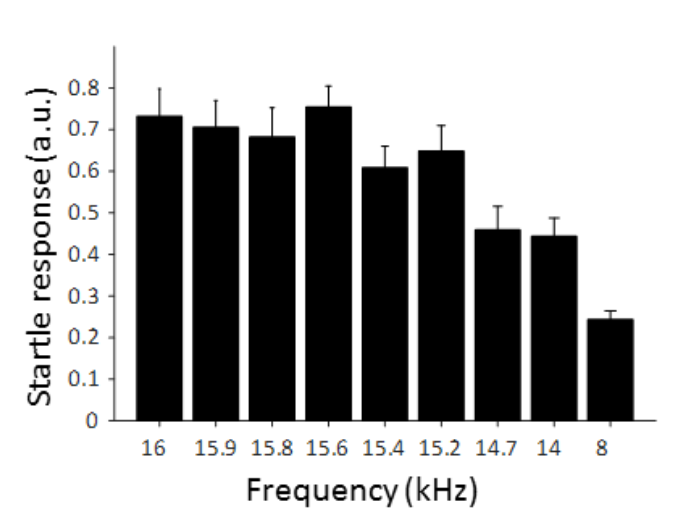

C

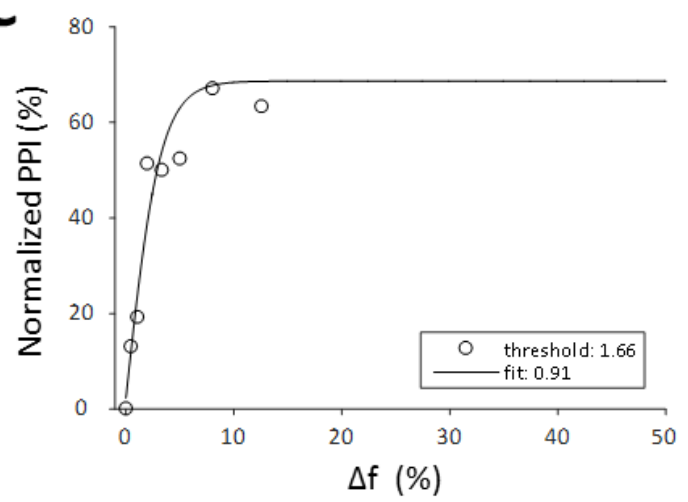

Figure 2.9: Inhibition of the ASR. A, Representative raw traces of all the trials during the PPI test. $B$, Example of the average ASR for each frequency tested. C, Inhibition of the ASR as function of the frequency change. Legend indicates the goodness of fit to a generalized logistic function and the threshold measured as the frequency change ( $\Delta \mathrm{f}$ in $\%$ ) that elicits $50 \%$ of the maximum inhibition.

\subsubsection{Latent Inhibition}

To study the effect of previous acoustic experience on subsequent learning, I used the latent inhibition paradigm in the Audiobox (de Hoz \& Nelken, 2014). Latent inhibition is a delay in learning due to previous exposure without conditioning to what later will be the conditioning stimulus. The animals were divided in 3 different groups and the experiment consisted on 4 phases: habituation, safe, pre-conditioned and conditioned. Only the $3^{\text {rd }}$ phase (pre-conditioned) was different for each group, while the other phases remained identical (Figure 2.10). 
1) In the habituation phase (3 days), no sound was presented and the sliding doors remained open allowing free water access;

2) In the safe phase (7 days), a safe tone of $8 \mathrm{kHz}$ was paired with visits to the corner and the sliding doors were open only after a nose-poke;

3) In the pre-conditioned phase ( 5 days), groups were exposed to different frequencies as follows: (i) for the control group, $71 \%$ of the visits were paired with an $8 \mathrm{kHz}$ tone and $29 \%$ were paired with a $4 \mathrm{kHz}$ tone; (ii) for the latent inhibition group, $71 \%$ of the visits were paired with an $8 \mathrm{kHz}$ tone and $29 \%$ were paired with a $16 \mathrm{kHz}$ tone; (iii) for the random group, $100 \%$ of the visits were paired with $8 \mathrm{kHz}$ and a $16 \mathrm{kHz}$ tone -played in the homecage- was paired to $29 \%$ of the visits of a mouse living in another Audiobox to its corresponding corner. All the sounds presented were safe for the animals to nose-poke and gain water access.

4) In the conditioned phase (5-12 days), during the first 5 hours, an $8 \mathrm{kHz}$ tone pip was paired with visits to the corner in $91 \%$ of the visits and $9 \%$ were paired with $16 \mathrm{kHz}$. For the rest of the phase the probabilities changed to 71 and $29 \%$, respectively. During this phase, mice had to learn to nosepoke only when they hear $8 \mathrm{kHz}$ (safe visit) and to avoid nose-poking when they hear $16 \mathrm{kHz}$ (conditioned visit). In case of a nose-poke during a conditioned visit, a $1 \mathrm{~s}$ air-puff was delivered through a hole locate above the drinking ports.

\subsubsection{Quantification of discrimination}

To assess discrimination performance, the discriminability index $\left(d^{\prime}\right)$ was calculated. $d^{\prime}$ used in signal detection theory is defined as:

$$
d^{\prime}=Z(H R)-Z(F A R)
$$

Where $Z(p), p \in[01]$, is the inverse of the cumulative of the Gaussian distribution, $H R$ is the hit rate, where a hit is the correct avoidance of a nose-poke during a conditioned visit, and FAR is the false alarm rate, where a false alarm is the avoidance of a nose-poke during a safe visit. Since d' cannot be calculated when either the hits or the false alarms reach levels of $100 \%$ or $0 \%$, in the few cases where this happened $99 \%$ and $1 \%$, respectively were used for these calculations. This manipulation reduced d' slightly (de Hoz \& Nelken 2014). 

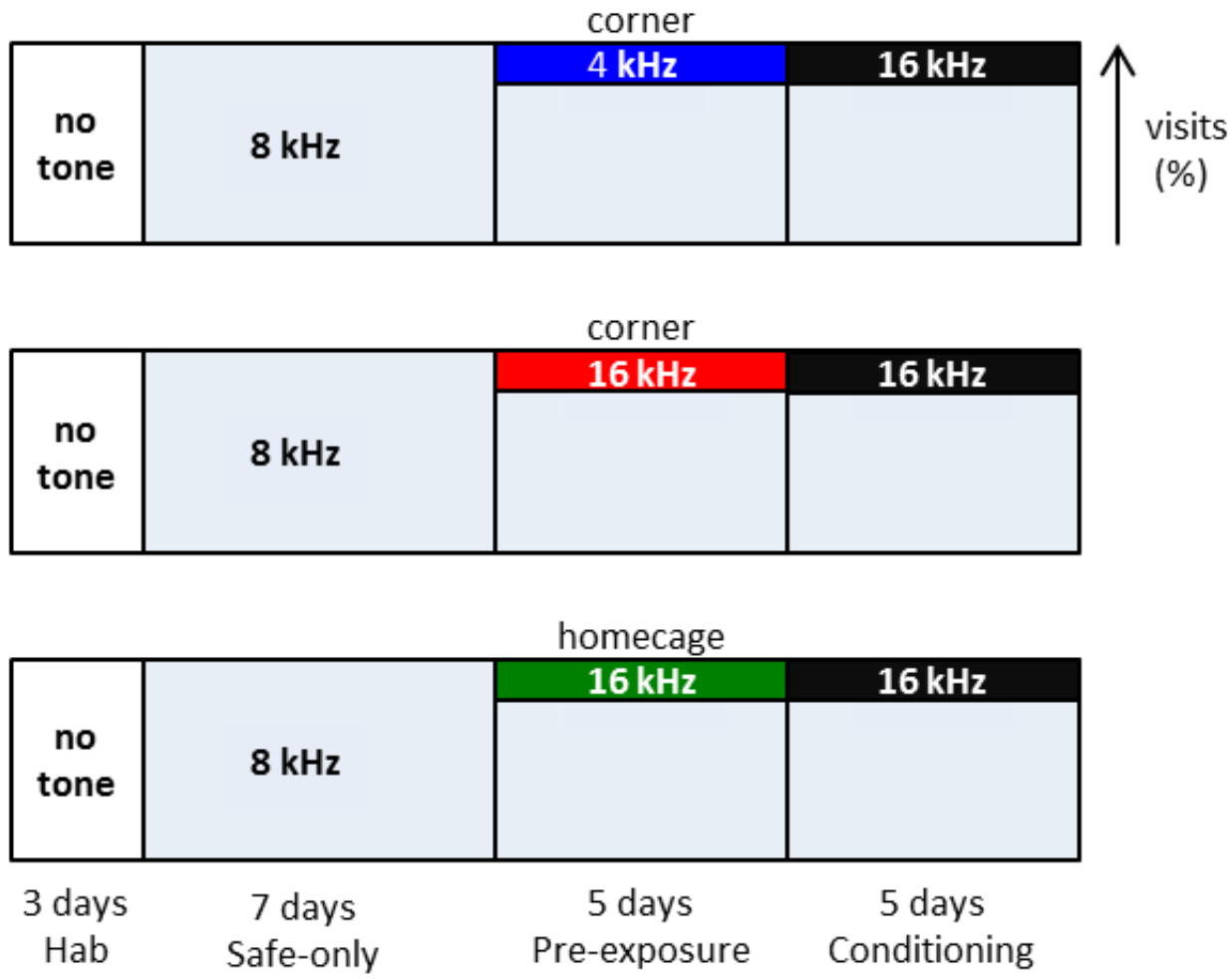

Figure 2.10: Latent inhibition. Scheme of the latent inhibition protocol for A, Control, B, Latent inhibition (red) and C, Random exposed groups. Boxes in color for each group indicate the frequency and location used for exposure during the pre-conditioned phase.

\subsection{Statistical analysis}

After testing for normality distribution using the Jarque-Bera test, group comparisons were made using multiple way ANOVAs, accordingly, followed by Bonferroni post-hoc tests. To analyze the cortical inactivation experiments a mixed design ANOVA with frequency, group and muscimol as fixed effects was used. For the immunohistochemistry data, where normality test failed, a Wilcoxon-signed rank test was used. To evaluate for significance in the temporal response matrices, a non-parametric two-sample test was used. The test uses permutations of group labels to estimate the null distribution, computed independently from each row as described by Glerean et al. 2015. The number of permutations was set to 10,000 for better estimation (a published Matlab script, bramila_ttest2_np, was adjusted for our data). To estimate the size differences between groups at each pixel of the matrix, the t-value, that represents the difference in units of standard error, was calculated. Means are expressed \pm SEM. Statistical significance was considered if $p<0.05$. 


\section{RESULTS}

\subsection{Electrophysiology}

To test whether behaviorally-relevant auditory experience during adulthood induces reorganization of frequency representation and changes in sound processing at the level of the inferior colliculus (IC), I performed acute electrophysiology and characterized auditory evoked responses in the IC of animals that lived for several days in groups in an enriched environment and heard a specific sound every time they entered the area where water was available. This environment is called the Audiobox, an automated behavioral apparatus that allows the monitoring of individual animals. Food is available ad libitum in a homecage space, while water is only available in a neighbor sound attenuated space where the animals can enter a 'corner', nose-poke in the hole and drink. Each mouse was exposed to tone pips of a specific frequency in every visit to the drinking corner (exposed group). I hypothesized that when pairing sounds with visits to the corner, an association between the acoustic stimulus and the environment of the corner is generated. The mice did not need to pay attention or respond to the sound but the sound was activated every time a mouse entered the drinking corner and for the duration of the visit. In the first experiment the pips had a frequency of $8 \mathrm{kHz}$. The control group consisted of age matched animals that lived during the same amount of time in the Audiobox without sound presentation. Mice lived in the Audiobox 24 hours a day for at least 5 days before acute electrophysiology began.

\subsubsection{Analysis of spectral tuning in the IC}

To analyze the effects of sound exposure on the spectral tuning of the IC, I performed acute electrophysiological recordings, where electrode penetrations were oriented dorso-ventrally and perpendicular to the isofrequency laminas and, therefore, along the tonotopic axis, and measured at each location the responses to different frequency-intensity combinations of sounds. In this initial experiment, the control group included 11 recorded sites from 4 animals, and the exposed group included 21 recording sites from 6 animals.

Examples of evoked activity for a control (Figure 3.1A) and an exposed (Figure 3.1B) mouse are represented in raster plots of spikes recorded in response to 5 trials per frequency for 24 frequencies, presented at $70 \mathrm{~dB}$. In this case, raster plots at different depths were not recorded simultaneously. For both mice, the dorso-ventral organization of the tonotopic map is evident with clusters of spikes appearing in response to progressively higher frequencies as depth increases in the IC (Figure 3.1). It is also evident that the evoked activity is stronger in the exposed group. To quantify the tuning in both groups, tuning curves were generated out of the spikes 


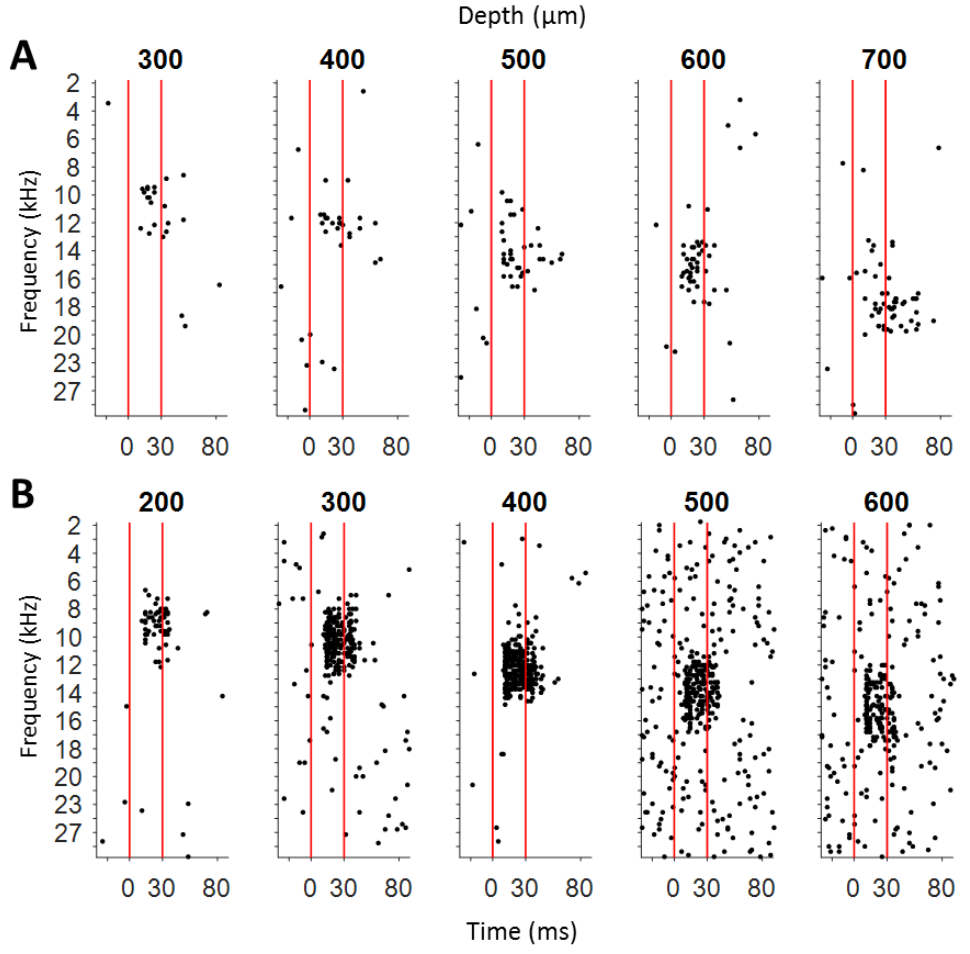

Figure 3.1: Evoked activity during 6 to 12 days of exposure to $8 \mathrm{kHz}$. Representative examples of raster plots recorded from different depths from $\mathbf{A}$, control; and $\mathbf{B}$, exposed animals. Each dot represents a recorded spike. Five repetitions of $30 \mathrm{~ms}$ tones of different frequencies were played at $70 \mathrm{~dB}$ SPL. Vertical red lines indicate the onset and offset of the tone. The recordings were obtained during 6 to 12 days of exposure to $8 \mathrm{kHz}$.

evoked, at $70 \mathrm{~dB}$, over an interval of $80 \mathrm{~ms}$ following stimulus onset (Fig. 3.2). The tuning curves of both the control (blue) and exposed (red) animals show that responses were larger in the exposed group. This was particularly evident in the area of the inferior colliculus that had best frequencies (peak of the tuning curve) near $8 \mathrm{kHz}$, the frequency used during behavior. The tuning curves in this graph also hint to something what will be one of the main findings of these series of experiments: for a given depth the best frequency of the exposed group tends to be higher than that of the control group. Note for example the difference in the peaks of the tuning curves at 400 microns depth. The statistical analysis showed that the tuning curves between groups were different at different depths (group effect, $F_{2}=8.43 p=0.0038$, group-depth interaction, $F_{1,6}=4.92 p=0.0001$ ). These results suggest, first that sound exposure induces an increased excitability in the exposed groups, and second that this happens mainly in the areas of the IC with a tuning around the exposed frequency.

To test the hypothesis that sound exposure induces frequency specific changes in restricted areas of the IC, I exposed a different group of animals to $16 \mathrm{kHz}$ in the corner, instead of $8 \mathrm{kHz}$. Since 16 $\mathrm{kHz}$ is represented more ventrally in the $\mathrm{IC}$, the changes in the amplitude of the tuning curves 


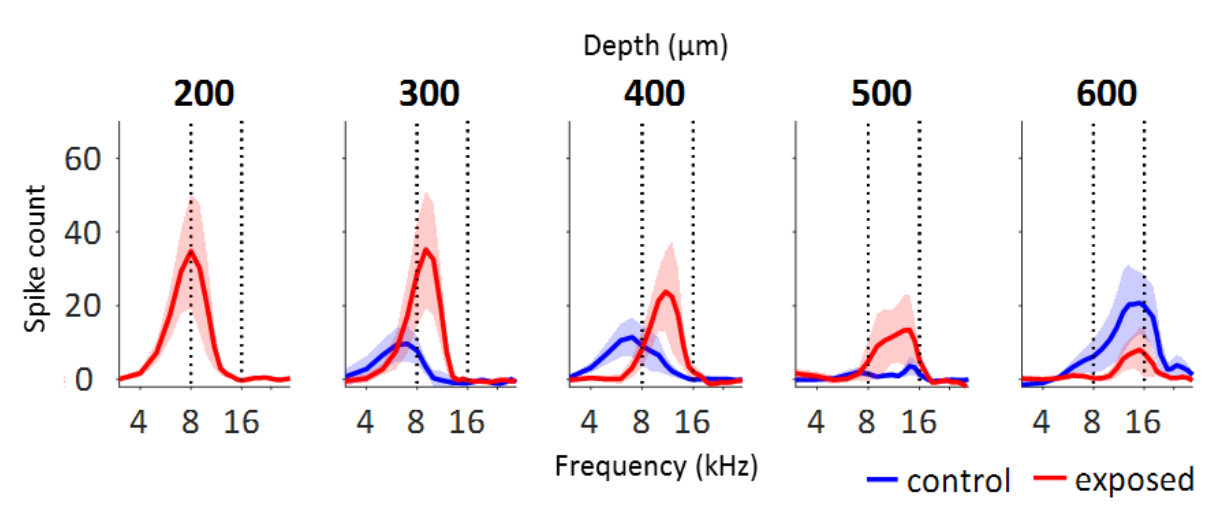

Figure 3.2: Effect of exposure to $8 \mathrm{kHz}$ on the spectral tuning of the IC. Average tuning curves at $70 \mathrm{~dB}$ for different depths in the IC for control (blue), and exposed (red) groups. The tuning curves were obtained by averaging the tuning curves of animals exposed for 6 to 12 days to $8 \mathrm{kHz}$.

would be expected to appear more ventrally than the locations modified by exposure to $8 \mathrm{kHz}$. In these experiments I used multi-electrode arrays that allowed me to record simultaneously 16 different depths spaced 50 microns apart. The most superficial electrode was always visible above the brain dura, making the depth assignment of the other electrodes very precise $( \pm 13 \mu \mathrm{m})$. All recording depths with significant responses (see Methods 2.4.4) were included in the analysis. The control group included 101 recording sites from 10 animals, and the exposed group included 168 sites from 15 animals. All animals lived in the Audiobox from 6-12 days.

Figure 3.3 represents again example raster plots of recordings performed in individual mice. Compared to the control animal (Figure 3.3A), the evoked responses of the exposed mouse (Figure 3B) were higher in multiple depths of the IC including the dorsal areas as noted before. The change was not restricted to areas with $\mathrm{BF}$ around $16 \mathrm{kHz}(600 \mu \mathrm{m})$ and, included depths comparable to those of the animals exposed to $8 \mathrm{kHz}$ (i.e. $300-400 \mu \mathrm{m}$ ). This pattern was typical for the mean of the groups (Figure 3.4, blue and red traces) since it can be observed in the tuning curves at many but not all depths (see for example 200, 350, 450 and $550 \mu \mathrm{m}$ ). The amplitude of the tuning curves of both groups, but specially the control group, showed zig zag type fluctuations along depths (Figure 3.4, blue and red traces). I believe this to be a true pattern since it can be observed in individual animals. By calculating the standard deviation across depths as a measure of dispersion, these fluctuations were more evident in the control group (7.06 vs 5.7 spikes, for control and exposed, respectively). Thus, it seems that activity is generally stronger in the exposed groups and that the increase is not restricted to the region with a $\mathrm{BF}$ comparable to that used in the behavioral exposure. 
The statistical analysis showed a significant difference between groups (group effect, $F_{2}=23.62$ $p=1 \times 10^{-6}$, group-depth interaction, $F_{1,13}=1.31 p=0.2$ ). To quantify the pattern of results, the 16
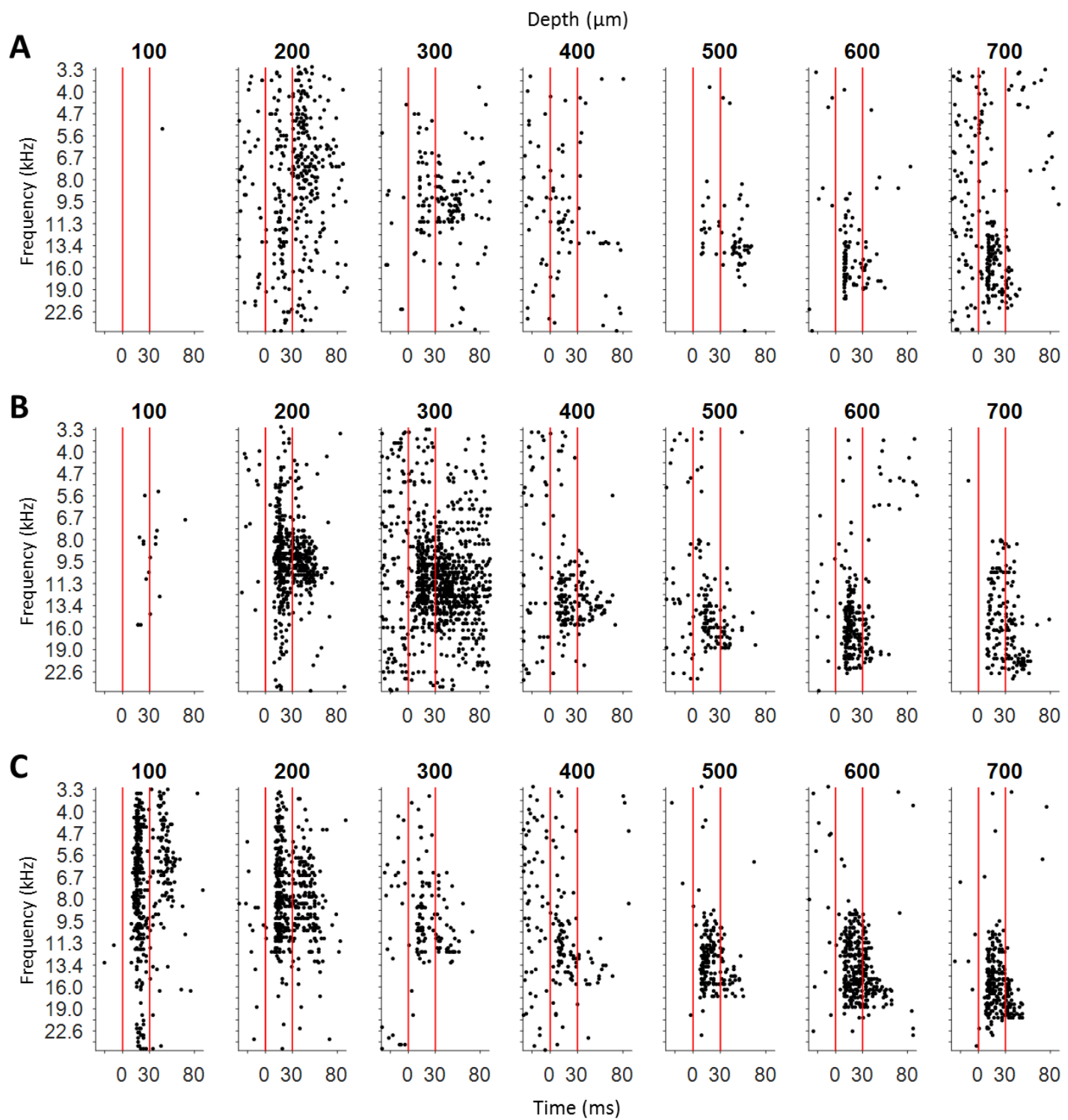

Figure 3.3: Evoked activity during 6 to 12 days of exposure to $16 \mathrm{kHz}$. Representative examples of raster plots recorded from different depths from A, one control; B, one relevant-exposed and C, one random animal. Each dot represents a recorded spike. Five repetitions of $30 \mathrm{~ms}$ tones of different frequencies were played at $70 \mathrm{~dB}$ SPL. Vertical red lines indicate the onset and offset of the tone. The recordings were obtained during 6 to 12 days of exposure to $16 \mathrm{kHz}$.

recorded locations were divided in 5 zones for statistical analysis. The first zone included the sites recorded from 100-150 $\mu \mathrm{m}$, since there is strong evidence that this area corresponds to the dorsal cortex overlaying the ICC (putative dorsal cortex). This region in mice was recently shown to have a thickness of $\sim 100 \mu \mathrm{m}$ (Barnstedt et al. 2015) and, as we will see in several of the tests performed here, it behaves differently from the rest of the colliculus. The rest of the zones were grouped in 
ranks of $150 \mu \mathrm{m}$ as following: $200-300$ (including both edges); 350-450; 500-600; and 650-750. The statistical analysis corroborated, the differences described between groups (group: $F_{2,4}=15.46$, $p<0.0001$; group-rank interaction: $\left.F_{2,4}=1.79 p=0.07\right)$. Therefore I compared groups at

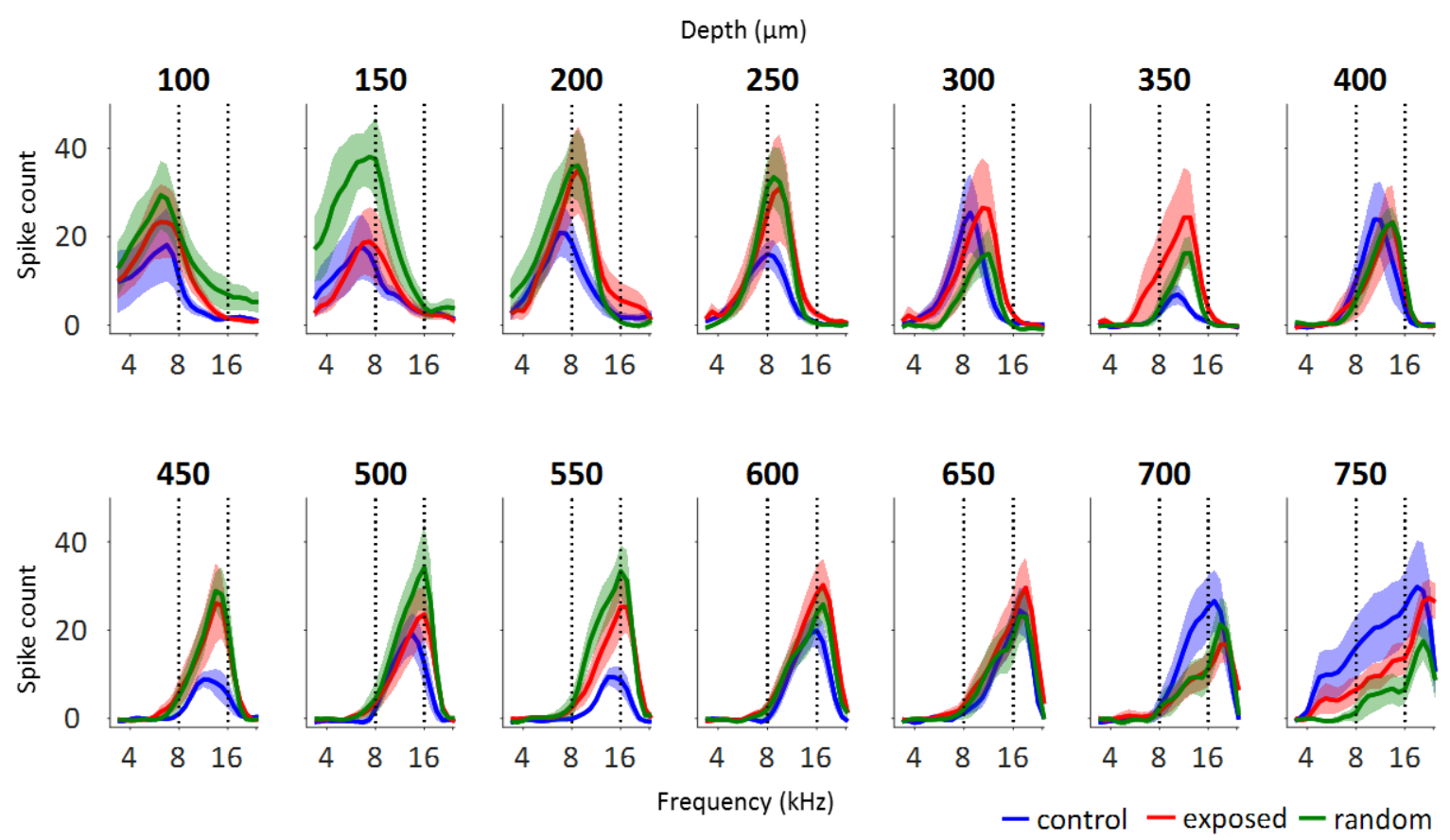

Figure 3.4: Effect of exposure to $16 \mathrm{kHz}$ on the spectral tuning of the IC. Average tuning curves at $70 \mathrm{~dB}$ for different depths in the IC for control (blue), relevant (red) and random (green) exposed groups. The tuning curves were obtained during 6 to 12 days of exposure to $16 \mathrm{kHz}$.

each individual rank. When compared to the control group, the average amplitude of the tuning curves at the locations tuned around $16 \mathrm{kHz}(500-600 \mu \mathrm{m})$ was larger in the exposed animals (group effect, $F_{1,13}=34.19 p=6 \times 10^{-9}$, group-frequency interaction, $\left.F_{1,13}=2.38 p=0.0002\right)$. Surprisingly, the effects of the exposure to $16 \mathrm{kHz}$ on spectral tuning extended beyond the area tuned to $16 \mathrm{kHz}$. More ventrally $(650-750 \mu \mathrm{m})$ the amplitude of the tuning curves was smaller (group effect, $F_{1,13}=7.99 p=0.0048$, group-frequency interaction, $F_{2}=1.02 p=0.4$ ). Surprisingly, in the dorsal zones (200-300 $\mu \mathrm{m}$ and 350-450 $\mu \mathrm{m}$ ) the tuning curves were also larger in the exposed group (200-300 $\mu \mathrm{m}$ : group effect, $F_{1,13}=13.12 p<0.001$, group-frequency interaction, $F_{2}=1.2 p=0.2 ; 350-450 \mu \mathrm{m}$ : group effect, $F_{1,13}=12.2 p<0.001$, group-frequency interaction, $\left.F_{2}=0.9 p=0.4\right)$. In the putative dorsal cortex there were no differences (group effect, $F_{1,23}=1.85 p=0.17$, group-frequency interaction, $\left.F_{2}=0.2 p=0.9\right)$.

Additionally, as already noticed in the previous experiment, the peak of the tuning curves of the exposed animals was shifted at multiple depths towards higher frequencies compared with the 
control group. To further characterize this possible change in frequency representation, the average $\mathrm{BF}$ at different depths between control and exposed animals were compared (Figure 3.5). The average BFs along the tonotopic axis were higher in animals exposed to $16 \mathrm{kHz}$ (Figure 3.5B). This increase in BF was evident from 200 to $750 \mu \mathrm{m}$. To quantify the change, I calculated the

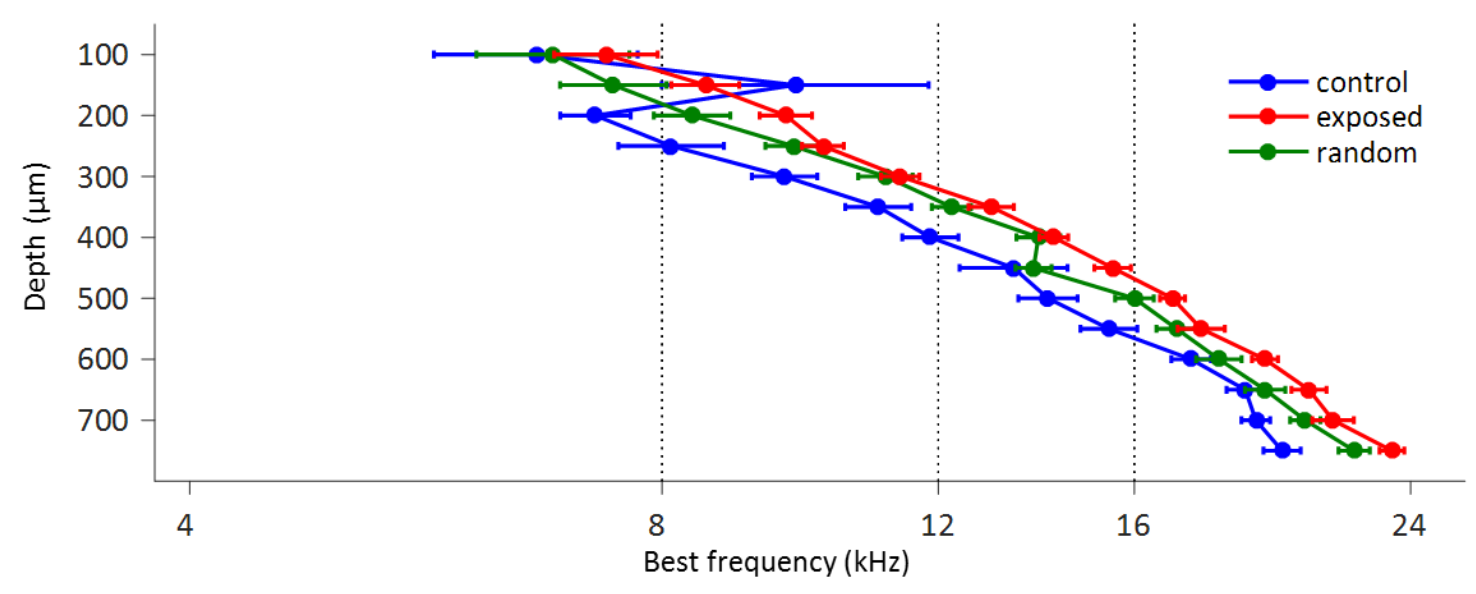

Figure 3.5: Effect of tone exposure on collicular tonotopy. Average best frequencies for different depths in the IC obtained during 6 to 12 days of sound exposure to $16 \mathrm{kHz}$ for control (blue), relevant (red) and random (green) exposed groups.

average difference in BF between control and exposed groups at each depth, and then averaged across depths, with the dispersion referring to the across depth variability ( $\Delta \mathrm{f} 1800 \pm 300 \mathrm{~Hz}$ along the axis, $F_{2,13}=34.56, p<0.0001$, group-depth interaction $F_{2,13}=1.48 ; p=0.06$, group comparisons $p<0.0001$ vs control). Together these results indicate that sound exposure can modify the spectral tuning in a non-specific manner by increasing the excitability in the IC. Furthermore, sound exposure can induce a frequency-unspecific shift in frequency representation towards higher frequencies along the measured length of the tonotopic map. The spatial extension of the effects in the IC seems to depend on the exposed frequency.

The previous results have shown that sound-exposure in the corner area of the Audiobox, triggered by visits of the animals, can lead to a frequency unspecific shift in frequency representation towards high frequencies and generates tuning curves with larger amplitudes in the low frequency range of the exposed sound, and lower amplitudes in the high frequency range of the IC of adult animals. The hypothesis is that collicular plasticity is induced because an association is generated between the exposed sound and the corner area. Thus, the sound acquires behavioral relevance. To confirm this hypothesis, it was required to compare the collicular tuning curves from animals exposed to the same sound, when this was not associated with anything in particular. For that purpose, a group of mice that lived in the Audiobox in similar conditions as the control group was exposed to $16 \mathrm{kHz}$ 
in the homecage area, instead of the corner as the exposed group. To ensure a similar pattern of exposure, a mouse living in another Audiobox, triggered the sound every time it visited its correspondent corner. This design prevented that the sound would be associated with a particular behavior (other than being in the homecage) or an outcome (such as water in the exposed group), while ensuring a similar pattern of sound exposure as in the exposed group. In these experiments, I used again multi-electrode arrays with 16 recording sites. From this group (random group) recordings were obtained from 92 sites and from 7 animals. All animals lived in the Audiobox from 6-12 days. In average, the animals from the exposed group and random group heard the tone during similar amount of time (1614 \pm 242 and $1330 \pm 232 \mathrm{~s}$ per day, respectively, $\mathrm{p}=0.275$ ), as calculated by the average daily length of visits to the corner (equivalent to exposure time to the sound) in both the animals of the relevant-exposed group and the mice that activated the sound in the homecage for the random group in the different replications, respectively.

Figure 3.3C shows examples of raster plots at different depths from a representative random mouse. The most superficial depths (100-200 $\mu \mathrm{m}$ that includes the putative dorsal cortex) showed a strong evoked activity that seems to gradually decrease to reach a minimum at $400 \mu \mathrm{m}$. More ventrally $(500-700 \mu \mathrm{m})$, strong evoked activity is recovered. It is clear that the evoked responses are larger at multiple depths than the control example (Figure 3.3A, see 100-200 and 500-600 $\mu \mathrm{m}$ ). Compared to the exposed example, the pattern of evoked activity is different in the dorsal-half of the IC $(100-400 \mu \mathrm{m})$. The activity of the random mouse is also larger at $100 \mu \mathrm{m}$, while the activity at $300-400 \mu \mathrm{m}$ is smaller than the exposed group. More ventrally, the activity between both examples is similar. The pattern previously described was similar for the group mean (Figure 3.4, green traces), tuning curves with large amplitudes in the putative dorsal cortex (100-150 $\mu \mathrm{m})$ that gradually decrease until reach a minimum at $350 \mu \mathrm{m}$, to further recover at $550 \mu \mathrm{m}$ and gradually decrease again at $750 \mu \mathrm{m}$.

I divided the recorded sites in zones, as previously described, and compared them with the control and exposed groups. Compared to the control group, the average amplitude of the tuning curves in the zone tuned to $16 \mathrm{kHz}(550-600 \mu \mathrm{m})$ was larger in the random animals (group effect, $F_{2}=10.71$ $p<0.0001$, group-frequency interaction, $\left.F_{2}=1.32 p=0.07 ; p<0.0001\right)$. More ventrally $(650-750 \mu \mathrm{m})$ the amplitude of the tuning curves was smaller (group effect, $F_{2}=11.10 p<0.001$, group-frequency interaction, $F_{2}=1.66 p=0.003$; group comparisons $p<0.0001$ ), as previously observed with the exposed group. The average tuning curves of the dorsal zones (200-300 and 350-450 $\mu \mathrm{m}$ ) were similar between groups (200-300 $\mu \mathrm{m}$ : group effect, $F_{2}=7.51 p<0.001$, group-frequency interaction, $F_{2}=0.79 p=0.8$; group comparisons, $p>0.05 ; 350-450 \mu \mathrm{m}$ : group effect, $F_{2}=7.26 p<0.001$, groupfrequency interaction, $F_{2}=0.73 p=0.9$; group comparisons $p>0.05$ ). In the zone of the putative dorsal 
cortex $(100-150 \mu \mathrm{m})$, the tuning curves were larger in the random group (group effect, $F_{2}=30.8$ $p<0.0001$, group-frequency interaction, $F_{2}=0.41 p=0.9$; group comparisons $p<0.0001$ ).

When compared to the exposed group, the average amplitude of the tuning curves in the zone tuned to $16 \mathrm{kHz}(550-600 \mu \mathrm{m})$ was similar (group comparison, $\mathrm{p}=0.1)$. More ventrally $(650-750 \mu \mathrm{m})$ the amplitude of the tuning curves was smaller (group comparison, $p=0.018$ ). The average tuning curves of the dorsal zones (200-300 and 350-450 $\mu \mathrm{m}$ ) was similar between groups (group comparison, $p>0.05$, for both zones). In the zone of the putative dorsal cortex (100-150 $\mu \mathrm{m})$, the tuning curves were larger in the random group (group comparison, $p<0.0001$ ).

These results indicate that random exposure also modifies the spectral tuning in the IC, with a different pattern to that observed after relevant sound exposure in the corner, mainly by inducing larger tuning curves in the area tuned to $16 \mathrm{kHz}$ and in the putative dorsal cortex, while decreasing the activity in the most ventral regions.

The observed increase in evoked activity in random and exposed groups could reflect an overall state of excitability in the IC. An indirect way to measure non-evoked excitability is by calculating the spontaneous activity (Bureš et al. 2014). I calculated the spontaneous activity by counting the spikes detected during $80 \mathrm{~ms}$ previous to tone onset, and average the spike counts across all trials in all the recording sites. Compared to the control group, the average spontaneous activity was significantly higher in the exposed group (Figure 3.6; $F_{2}=5.57, p=0.004 ; 2.35 \pm 0.28$ vs $4.32 \pm 0.47$ spikes, control and exposed group, respectively, group comparisons, $p=0.0032$ ). Compared to the random group, the spontaneous activity of the exposed group was higher but not significantly (4.32 \pm 0.47 vs $3.38 \pm 0.32$ spikes $p=0.2$, for exposed and random groups respectively). Finally, the average spontaneous activity of the random group was higher than the control but not significantly $(2.35 \pm$ 0.28 vs $3.38 \pm 0.32$ spikes $p=0.4$ ). This result suggests that sound exposure increases overall excitability in the IC. Since spontaneous activity was subtracted from the spike count used to generated the tuning curves and calculate the frequency shifts, this increase is not responsible for the pattern of results described above.

To characterize if random exposure also induces changes in frequency representation, the average $\mathrm{BF}$ at different depths of the random group was compared to the control and exposed groups. The average BFs along the tonotopic axis were higher in random animals compared to controls (Figure 3.5, green trace; $\Delta f 900 \pm 364 \mathrm{~Hz}$ along the axis, $p=0.0011$ vs control). Compared to the exposed group the average shift of the random group along depth was half of the shift of the exposed group ( $\Delta \mathrm{f} 900 \pm 364 \mathrm{~Hz}$ vs $1800 \pm 364 \mathrm{~Hz}$, for random and exposed groups respectively, $p=0.0001$ ). 


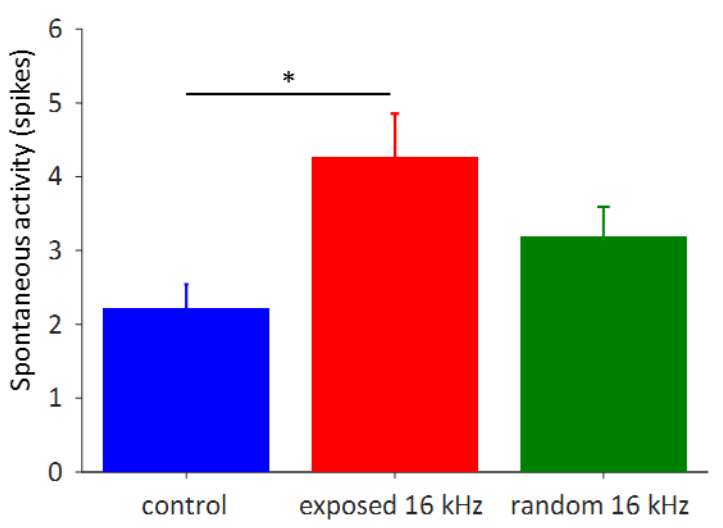

Figure 3.6: Effect of tone exposure on the spontaneous activity in the IC. Average spontaneous activity per trial during 6-12 days of sound exposure for control, exposed and random groups. * $\mathrm{p}<0.05$.

Together, these results indicate that random and behaviorally-relevant exposure generate plasticity in the IC of adult animals. Mainly an increase in excitability as observed by a non-specific increase in the spontaneous and evoked activity and a shift in the frequency representation towards high frequencies. However, the locations along the IC where the increase in evoked activity occurred were not identical between exposure paradigms. The most striking difference was the increase in evoked activity in the putative dorsal cortex of the random group. Additionally, the shift in frequency representations was stronger in the exposed group, suggesting an additive effect of behavioral relevance to sound exposure.

\subsubsection{Analysis of temporal firing patterns in the IC}

The next question I asked was whether in addition to the induced changes in spectral tuning and frequency representation, sound exposure could also modify the temporal firing pattern in neurons of the IC. Changes in the temporal firing pattern could indicate that the coding properties of collicular neurons are susceptible of plastic changes (Quian Quiroga \& Panzeri 2009; Panzeri et al. 2010). I was particularly interested in analyzing the temporal firing pattern evoked by the sound used during behavior $(16 \mathrm{kHz})$. For this analysis I selected the recorded locations with a $\mathrm{BF}$ of 16 $\mathrm{kHz} \pm 0.25$ octaves. If more than one location was found per animal, an average of the responses was made before including it in the group dataset. The data analyzed was the same as the one used in the spectral tuning analysis of animals exposed to $16 \mathrm{kHz}$.

First, for each group, from the selected recorded locations, I built an average PSTH with 5 ms bins from the response to $16 \mathrm{kHz}$ at $70 \mathrm{~dB}$. The average depths of the recording sites selected for the 

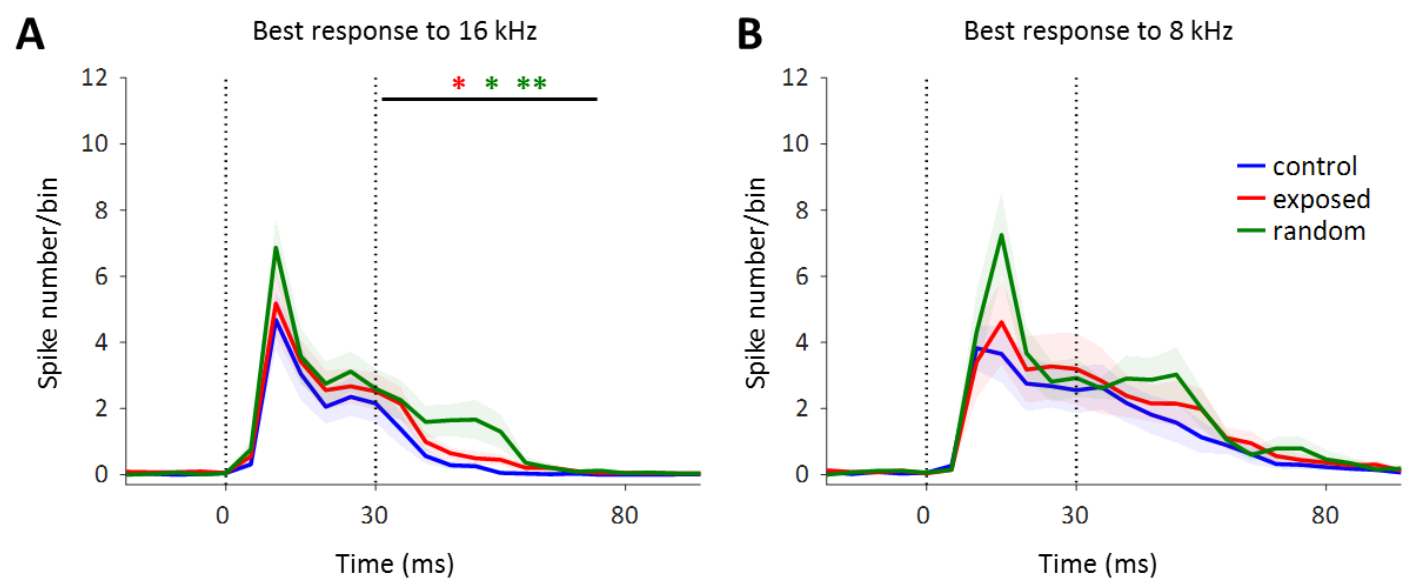

Figure 3.7: Effect of tone exposure on the firing pattern of the IC. Average PSTH ( $5 \mathrm{~ms}$ bins) of the depth with the best responses for control (blue), relevant (red) and random (green) exposed groups to $\mathbf{A}, 8 \mathrm{kHz}$; and $\mathbf{B}, 16 \mathrm{kHz}$ at $70 \mathrm{~dB}$. The responses were obtained during 6 to 12 days of exposure to $16 \mathrm{kHz}$. Vertical dashed lines indicate tone onset and delayed. ${ }^{*} p<0.05$, exposed vs control; ${ }^{*} p<0$ .05 exposed vs random; ${ }^{*} \mathrm{p}<0.001$ random vs control;

analysis were: $601 \pm 72 \mu \mathrm{m}$ for the control group, and $473 \pm 57 \mu \mathrm{m}$, for the exposed group. As it can be appreciated in the figure $3.7 \mathrm{~A}$, the average pattern of the response to $16 \mathrm{kHz}$, between control (blue trace) and exposed groups (red trace) during sound duration (represented by the vertical dashed lines) was very similar. After sound offset, there was a sustained increase in activity from 40 to $60 \mathrm{~ms}$ in the exposed group compared to the control group. For statistical comparisons, the responses were divided in onset (0-30 ms after sound onset) and delayed (31-80 ms after sound onset), thus there was no difference in the onset component, while the delayed component was significantly larger than the control group $(p=0.03)$. These results indicate that sound exposure mildly changes sound processing by increasing the delayed response.

Next, to evaluate if the temporal firing could affect the responses to other sounds in a similar nonspecific way as the changes previously described for spectral tuning and frequency representation, I analyzed in the same way the responses to $8 \mathrm{kHz} \pm 0.25$ octaves, an octave below the exposed sound. The average depths of the recording sites selected for this analysis were: $236 \pm 51 \mu \mathrm{m}$ and $147 \pm 54 \mu \mathrm{m}$, for the control and exposed groups, respectively. The averaged PSTH of the exposed group and control group was very similar in magnitude and in the pattern of the response (Figure 3.7B). There were no significant differences in the onset and delayed components of the response.

As with the previous analysis of spectral tuning and frequency representation, to evaluate the effect of sound exposure without behavioral relevance, I analyzed the temporal firing pattern to $16 \mathrm{kHz} \pm$ 
A

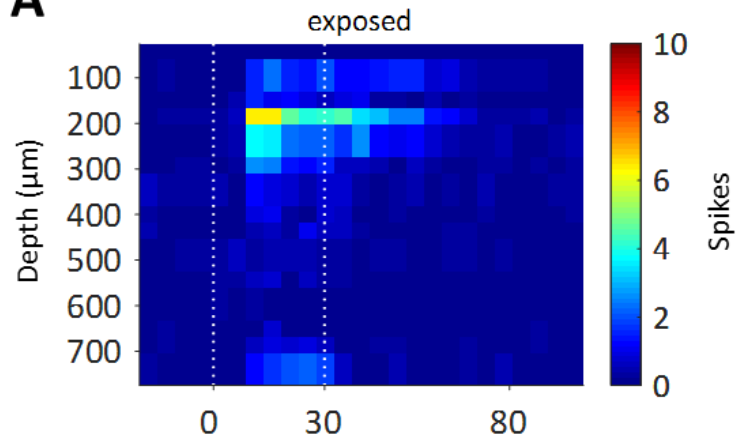

C

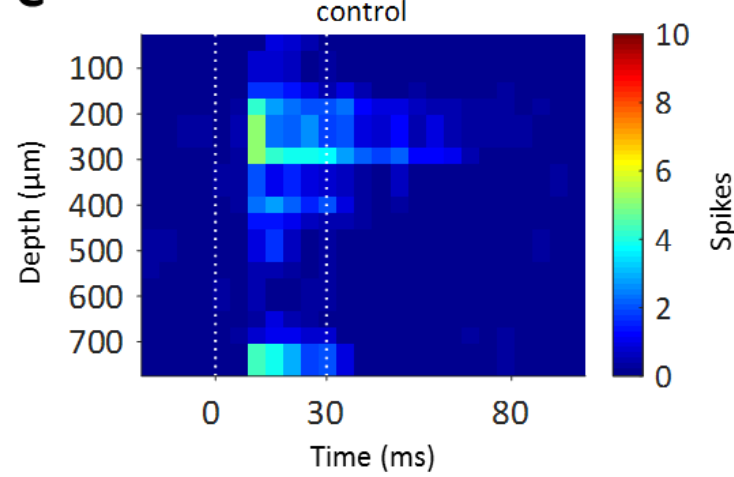

B

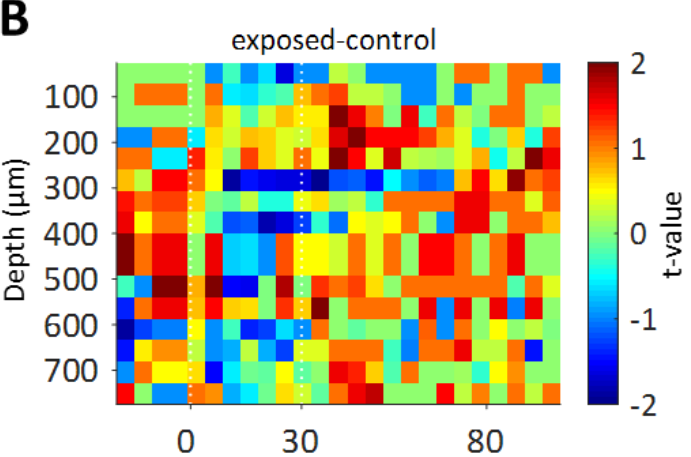

D

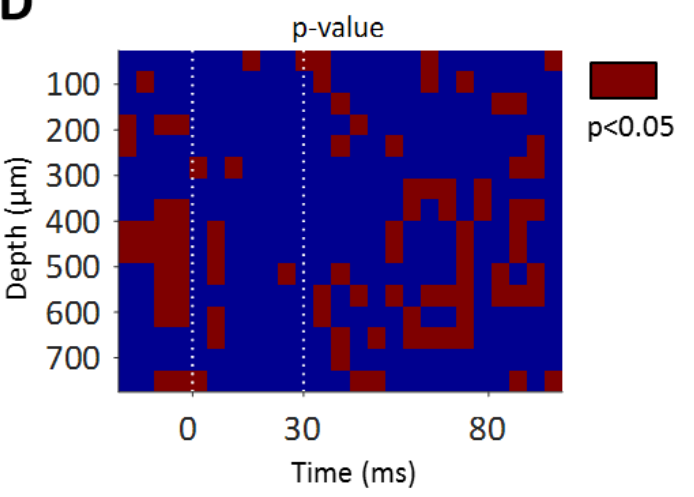

Figure 3.8: Effect of relevant tone exposure on the spatio-temporal firing pattern to $8 \mathrm{kHz}$ in the IC. Average temporal response areas ( $5 \mathrm{~ms}$ bins) of the simultaneous response, along the dorsoventral axis of the IC, to $8 \mathrm{kHz}$ at $70 \mathrm{~dB}$ for A, Relevant exposed to $16 \mathrm{kHz}$ and $\mathbf{C}$, Control groups. B, Difference in activity between exposed and control groups normalized by the error standard. $\mathbf{D}$, Pixels in the matrix with a significant difference $(p<0.05)$. Recordings were obtained during 6 to 12 days of sound exposure.

0.25 octaves of the random group exposed to $16 \mathrm{kHz}$. The average depth of the recording locations used in this analysis was $531 \pm 41 \mu \mathrm{m}$. Interestingly, in the random group there was a slight increase in the amplitude of the peak response (Figure 3.7A, green trace). Also, it had sustained larger response that finished around $60 \mathrm{~ms}$ after sound onset. Compared to the control group, the onset component of the response was not significantly different, but the late component was highly significant $(p=0.0001)$. When compared to the exposed group, there were no differences in the onset component as well, while the delayed component was significantly larger in the random group ( $p=0.01$ ), indicating that random exposure also modifies the temporal firing pattern to the exposed sound.

Finally, I analyzed and compared the temporal firing pattern to $8 \mathrm{kHz}$ of the random group. The peak response was slightly higher than the control and exposed groups. (Figure 3.7B, green trace), and the delayed component also showed a tendency to have a larger amplitude. However, these 
differences did not reach significance. Together, these results indicate that relevant and random sound exposure
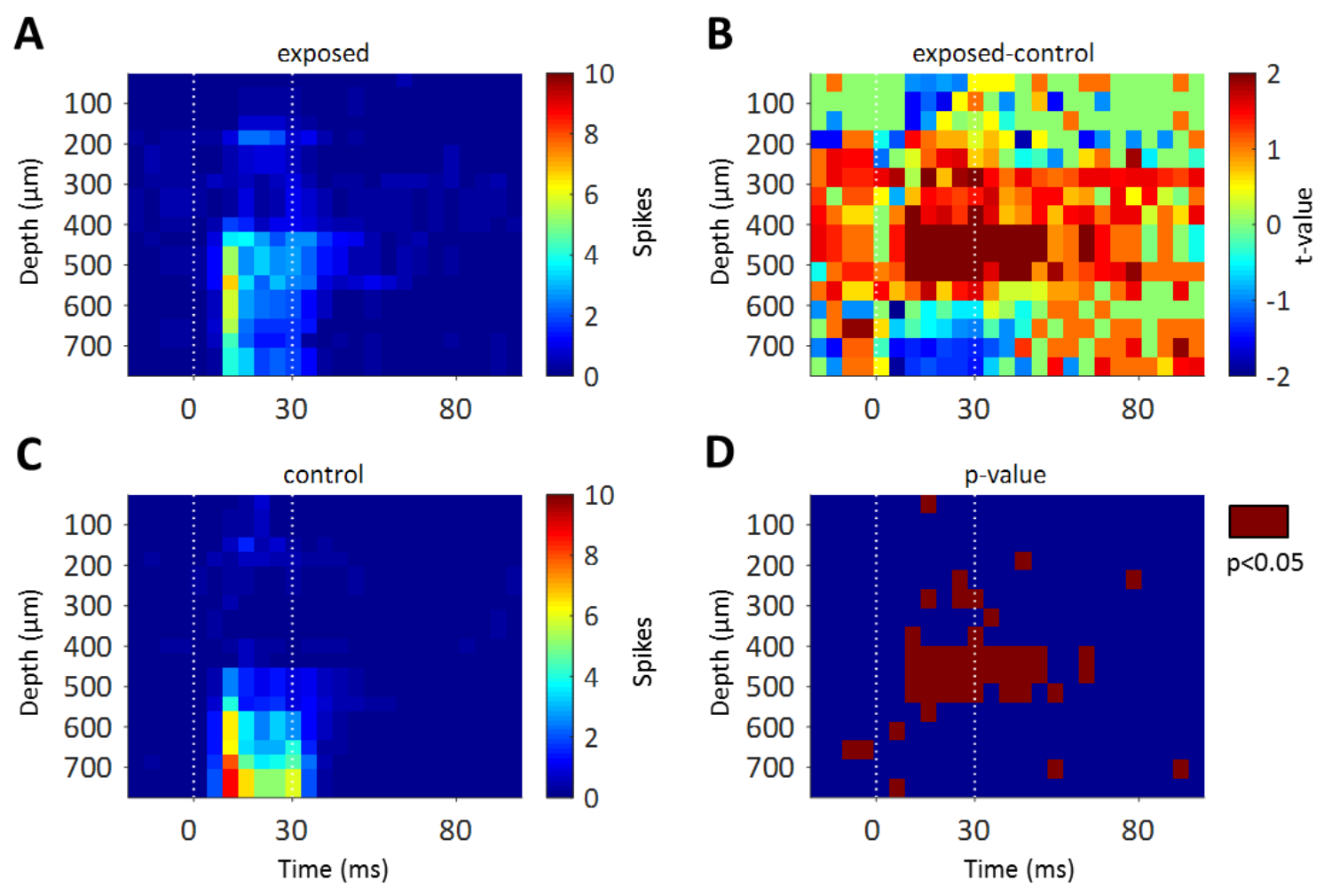

Figure 3.9: Effect of relevant tone exposure on the spatio-temporal firing pattern to $16 \mathrm{kHz}$ in the IC. Average temporal response areas ( $5 \mathrm{~ms}$ bins) of the simultaneous response, along the dorsoventral axis of the IC, to $16 \mathrm{kHz}$ at $70 \mathrm{~dB}$ for $\mathbf{A}$, Relevant exposed to $16 \mathrm{kHz}$ and $\mathbf{C}$, Control groups. B, Difference in activity between exposed and control groups normalized by the error standard. D, Pixels in the matrix with a significant difference $(p<0.05)$. Recordings were obtained during 6 to 12 days of sound exposure.

do not modify the pattern of responses in the IC. However, sound exposure induced a significant increase of the delayed response. The magnitude of the increase was higher in the random group than in the exposed group. Moreover, this increase was observed only at the frequency of the exposed tone (16 kHz), suggesting a frequency specific effect.

What is the spatial profile of the responses to a sound along the dorso-ventral axis of the IC? It is important to keep in mind that, even though frequencies are best represented at particular depths, they still can evoke responses at depths different from their preferred depth. Since in these experiments simultaneous activity was recorded using multi-electrode arrays, I could address this question. For this purpose, I built temporal response matrices using the PSTH of a specific frequency recorded simultaneously at different depths. For this analysis, I built the temporal response matrices to 16 and $8 \mathrm{kHz}$. The Figure $3.8 \mathrm{C}$, shows the average temporal response area to $8 \mathrm{kHz}$ of 
the control group. Each pixel represents the average spike count (the larger the spike count the warmer the color) of a depth-time combination. As it can be appreciated there is a cluster of pixels in the most

A

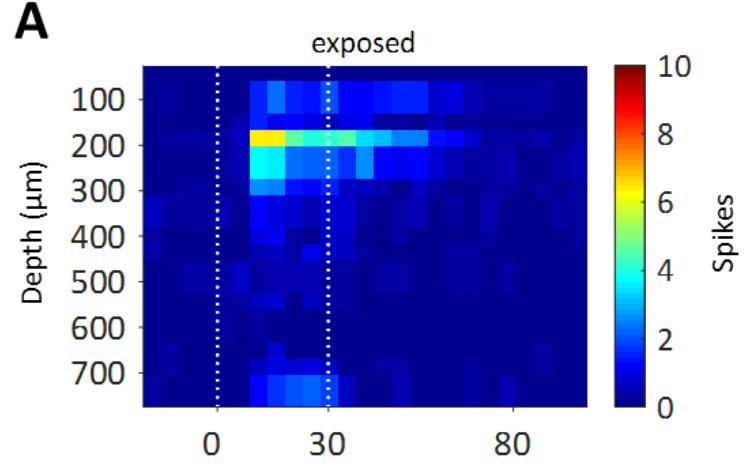

C

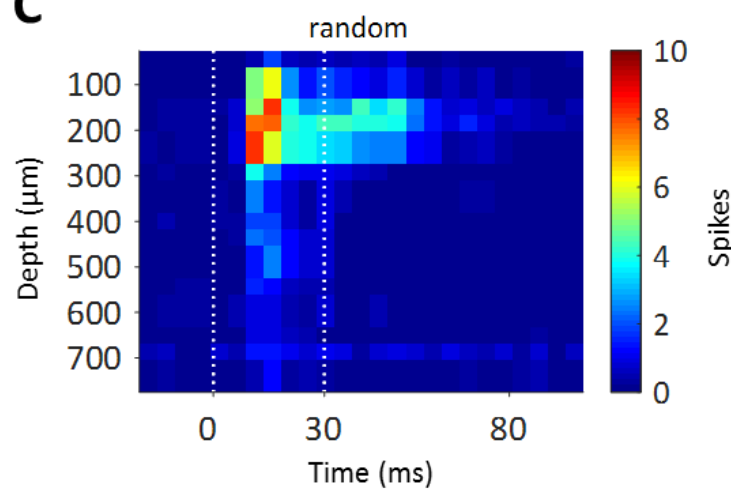

B

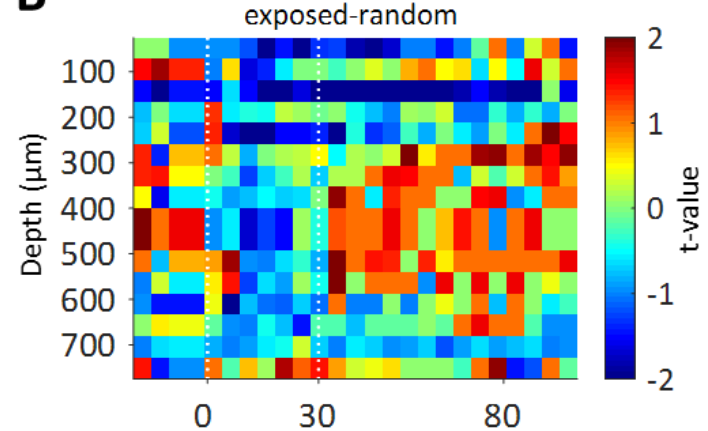

D

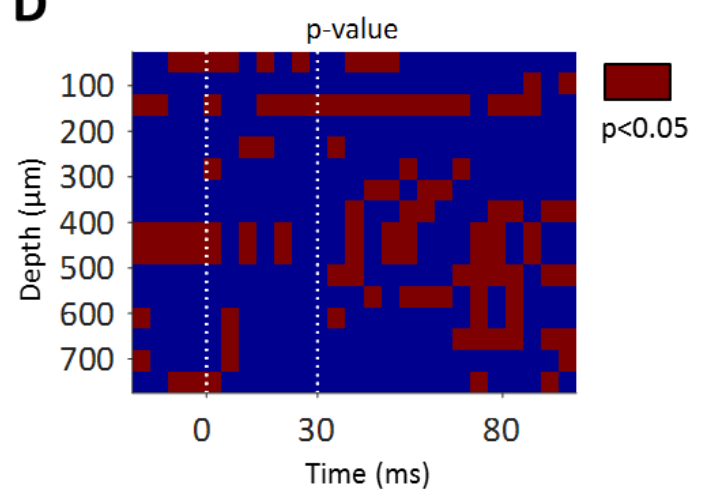

Figure 3.10: Effect of relevant and random tone exposure on the spatio-temporal firing pattern to $8 \mathrm{kHz}$ in the IC. Average temporal response areas ( $5 \mathrm{~ms}$ bins) of the simultaneous response, along the dorso-ventral axis of the IC, to $8 \mathrm{kHz}$ at $70 \mathrm{~dB}$ for $\mathbf{A}$, Relevant and $\mathbf{C}$, Random groups exposed to $16 \mathrm{kHz}$. B, Difference in activity between exposed and control groups normalized by the error standard. $\mathbf{D}$, Pixels in the matrix with a significant difference $(p<0.05)$. Recordings were obtained during 6 to 12 days of sound exposure.

dorsal area that represent the response to $8 \mathrm{kHz}$, with the warmest pixel (peak response) located at $300 \mu \mathrm{m}$ for the control group. In contrast, the average temporal response area to $8 \mathrm{kHz}$ of the exposed group (Figure 3.8A) shows that the peak response (warmest pixel) is located more dorsally, at $200 \mu \mathrm{m}$. This result was consistent with the shift in BFs of the tonotopic axis. Group comparisons were made using the difference in the responses at each pixel normalized by the standard error ( $t$ value, see Methods 2.1). The differences in the response to $8 \mathrm{kHz}$, between exposed and control group are in Figure 3.7B and the p-values in Figure 3.8D. A warmest color indicates the exposed group had larger spike counts, while a colder color indicates that the control group had a larger spike counts. 
Next, I evaluated the response to the tone used during behavior: $16 \mathrm{kHz}$. The average temporal response area of the control group (Figure 3.9C) revealed that the response to $16 \mathrm{kHz}$ covered the most ventral part measured in the IC $(500-750 \mu \mathrm{m})$, spanning a distance of $\sim 250 \mu \mathrm{m}$. The average temporal response area to of the exposed group (Figure 3.9A) showed that the response to $16 \mathrm{kHz}$
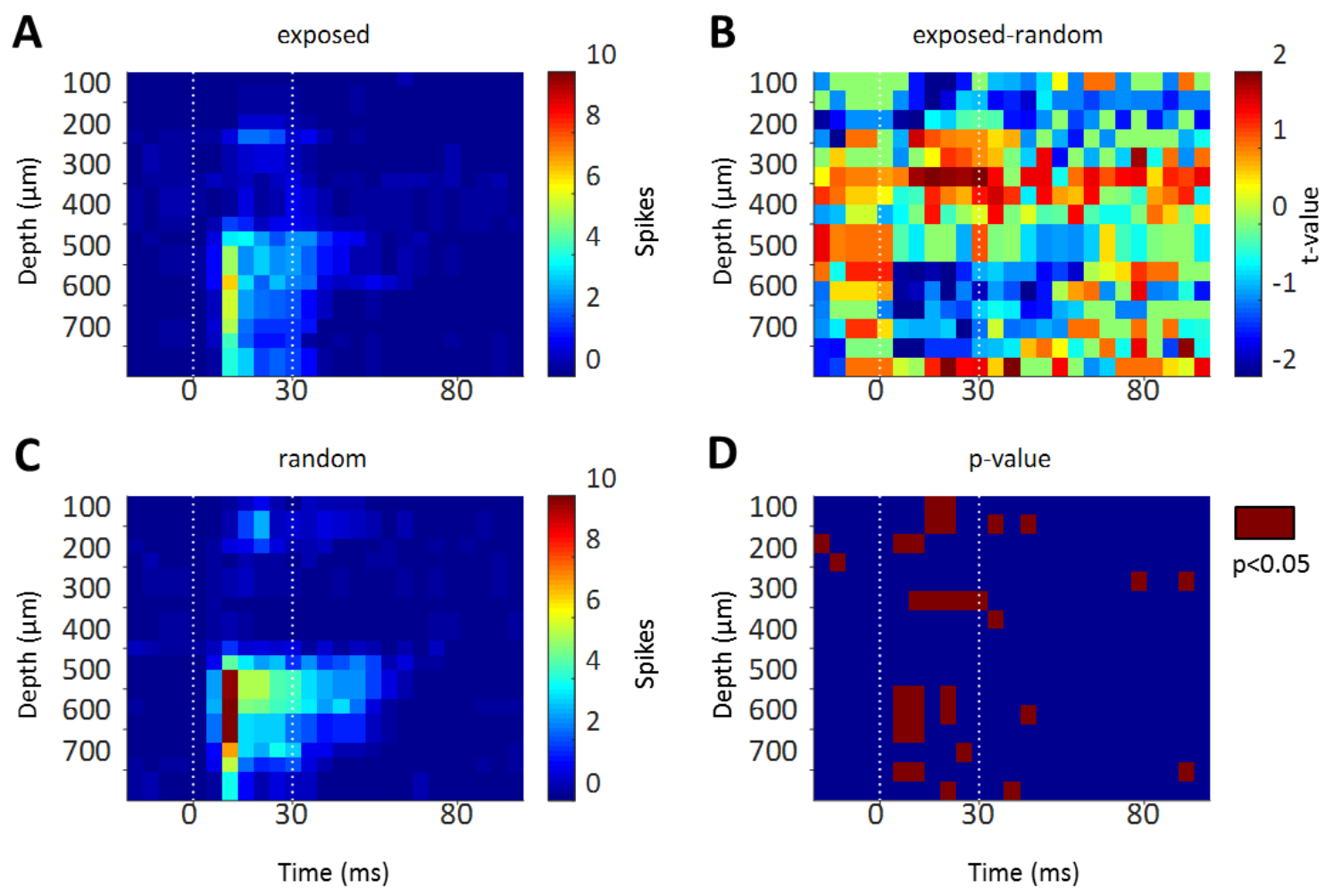

Figure 3.11: Effect of relevant and random tone exposure on the spatiotemporal firing pattern to $16 \mathbf{~ k H z}$ in the IC. Average temporal response area( $5 \mathrm{~ms}$ bins) of the simultaneous response, along the dorso-ventral axis of the IC, to $16 \mathrm{kHz}$ at $70 \mathrm{~dB}$ for $\mathbf{A}$, Relevant and $\mathbf{C}$, Random groups exposed to $16 \mathrm{kHz}$. B, Difference in activity between exposed and control groups normalized by the error standard. $\mathbf{D}$, Pixels in the matrix with a significant difference $(p<0.05)$. Recordings were obtained during 6 to 12 days of sound exposure.

covered the medial and ventral areas of the IC $(400-750 \mu \mathrm{m})$, covering a distance of $350 \mu \mathrm{m}$. Consequently, the evoked activity between 400-550 $\mu \mathrm{m}$ was higher in the exposed group, while the activity from 650 to $750 \mu \mathrm{m}$ was lower (Figure 3.9B-D). These results indicate that the responsive distance to the exposed sound is expanded and shifted after sound exposure. Identical analysis was made for the random group. The average temporal response area to $8 \mathrm{kHz}$ of the random group (Figure $3.10 \mathrm{C}$ ), revealed that the response to $8 \mathrm{kHz}$ covered the most dorsal part of the IC (100-400 $\mu \mathrm{m})$, including the putative dorsal cortex, covering a distance of $\sim 300 \mu \mathrm{m}$. When compared to the exposed group (Figure 3.10A), the evoked activity was larger in all this area (Figure 3.10D). 
The average temporal response area to $16 \mathrm{kHz}$ of the random group (Figure 3.11C) showed that the response to $16 \mathrm{kHz}$ covered the ventral part of the IC $(500-750 \mu \mathrm{m})$, a distance of $\sim 250 \mu \mathrm{m}$, similar to the control group. When compared to the exposed group the responsive distance was smaller (Figure $3.11 \mathrm{~A} ; 250$ vs $350 \mu \mathrm{m}$ ). However, the onset responses of the random group were stronger in the ventral areas of the IC and smaller more medially (Figure 3.11B,D). These results indicate that relevant and random sound exposure alters the spatial response profile in the IC in different ways. For the exposed group, the peak response to $16 \mathrm{kHz}$ was located more dorsally and there was an increase in the depths that responded to $16 \mathrm{kHz}$; while only a shift (more dorsally vs control) in the peak response, without increase in the responsive area, was found in the responses to $8 \mathrm{kHz}$. For the random group there was a shift (also dorsal) in the peak response to $16 \mathrm{kHz}$, but not an expansion; while the response area to $8 \mathrm{kHz}$ was expanded compared to the exposed group. In the figures it is interesting that often the patches of significant differences are in areas that do not have a proper response per se. In the exposed groups it looks like there is a faint background response everywhere.

\subsubsection{Two-dimensional organization of frequency representation in the IC}

The anatomical organization of frequency representation in the IC are the frequency laminas, that extend in the rostro-caudal axis with an inclination of $\sim 45$ degrees (Figure 3.12A). For example, in the rostral part $16 \mathrm{kHz}$ is represented more ventrally while in the caudal part it is represented more dorsally (Figure 3.12A, e.g. red line of the scheme). I hypothesized that the shift in frequency representation towards high frequencies, caused more dramatically by relevant-sound exposure, should affect the whole extension of the frequency lamina rather than discrete areas.

To test this hypothesis, I performed recordings using time multi-electrode arrays arranged in 4 shanks with 4 electrodes per shank. The penetrations of the electrode were oriented dorsoventrally as in previous recordings (Figure 3.12A). In this experiment, I recorded animals from the control and exposed group and compared BF distribution at different depths along the rostrocaudal axis. The control group consisted of 287 recording sites from 6 animals and from the exposed group consisted of 306 recording sites from 7 animals.

The average BF distribution for each shank at multiple depths from control (dashed lines) and exposed animals (continuous line) is shown in Figure 3.12B. It can be appreciated that the dashed lines (averaged BF of control animals) are always to the left of their color matched continuous lines (average BF of exposed animals) indicating that, along the rostro-caudal axis of the IC, control animals have a frequency distribution with lower frequencies than exposed animals. 
To better understand the frequency laminas' distribution, we color coded the BF at each depth in a separate color dot-plots for each group (Figure 3.12C-D). In these plots lower frequencies have a colder color and high frequencies a warmer color, the BF of $16 \pm 1 \mathrm{kHz}$ were colored black for better appreciation. In the control group (Figure $3.12 \mathrm{C}$ ), the reported inclination of the $16 \mathrm{kHz}$ frequency lamina can be easily appreciated (Stiebler \& Ehret 1985). In the shank that recorded the rostral portion of the IC (rostral), the BF of $16 \mathrm{kHz}$ appears at $750 \mu \mathrm{m}$, and move gradually upwards until it

A

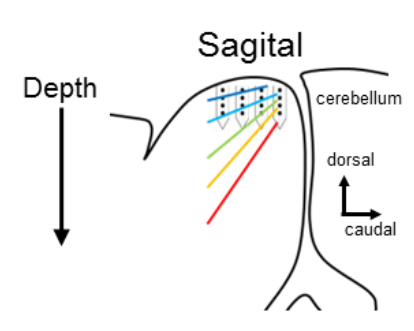

B

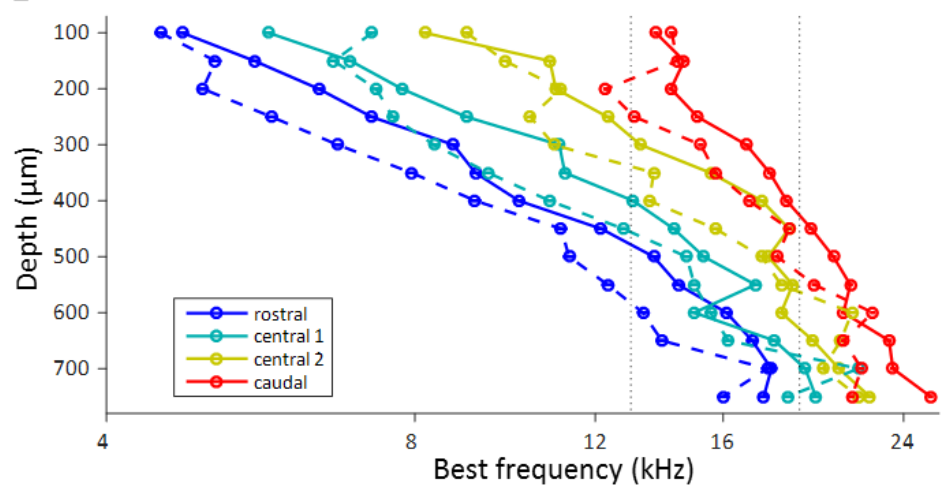

D

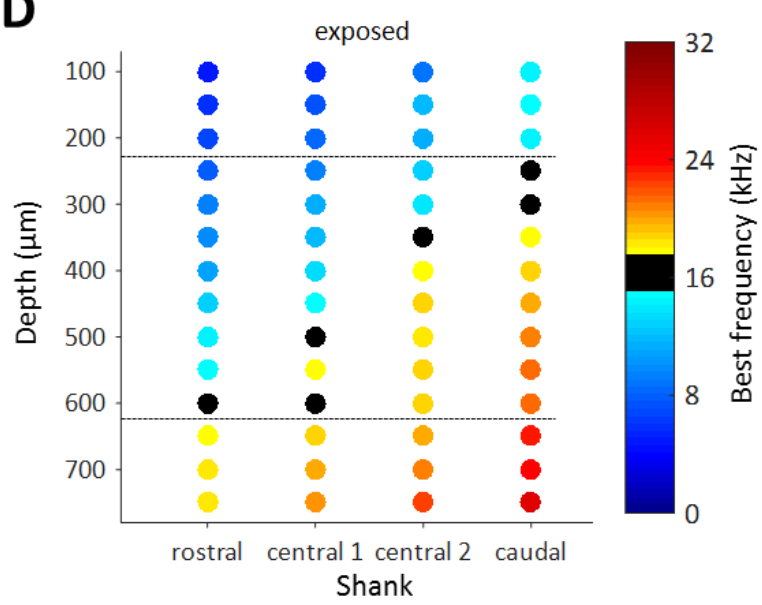

Figure 3.12: Effect of tone exposure on the tonotopic organization at the rostro-caudal and dorsoventral axes of the IC. A, Representation of a sagital section of the IC that shows the anatomical distribution of the isofrequency laminas (different transversal color lines) and the recording approach using 4 shanks multi-electrode arrays. B, Average best frequencies along the dorsoventral axis at different rostro-caudal locations obtained during 6 to 12 days of sound exposure to $16 \mathrm{kHz}$ for control (dashed lines) and exposed (continuous lines) groups. Error bars are omitted for clarity. C, Distribution of average best frequencies in the dorso-ventral axis for each recording shank of the control group. D, Distribution of best frequencies in the dorso-ventral axis for each recording shank of the exposed group. For $C$ and $D$, the black dots represent best frequencies of $16 \pm 1 \mathrm{kHz}$ and the horizontal dashed lines the upper and lower limits of the corresponding depths for the exposed group. 
reaches its most dorsal position in the caudal part $(300 \mu \mathrm{m})$. It also occurs with the exposed group, however the depth in the rostral part where $16 \mathrm{kHz}$ is the $\mathrm{BF}$ is located more dorsally (600 $\mu \mathrm{m})$. This shift upwards is maintained along the rostro-caudal axis until the caudal part $(250 \mu \mathrm{m})$. The stronger shift of the exposed animals occurred in the rostral and caudal extremes of the IC. In the rostral part, the average BF shift (calculated as in section 3.1.1) along the dorso-ventral axis was $\Delta f 1,468$ $\pm 205 \mathrm{~Hz}\left(F_{1,13}=6.24, p=0.013\right.$, group-depth interaction $\left.F_{1,13}=0.12 ; p=0.99\right)$. In the caudal part, the average $B F$ shift along the dorso-ventral axis was $\Delta f 1,263 \pm 367 \mathrm{~Hz}\left(F_{1,13}=5.23, p=0.02\right.$, group-depth interaction $\left.\mathrm{F}_{1,13}=0.38 ; \mathrm{p}=0.97\right)$. The central portions of the IC also showed a shift in $\mathrm{BF}$ but lower in magnitude (central1, a shift of $874 \pm 371 \mathrm{~Hz}, \mathrm{~F}_{1,13}=2.14, p=0.14$, group-depth interaction $\mathrm{F}_{1,13}=0.35$; $p=0.98$; central2, a shift of $771 \pm 445 \mathrm{~Hz}, F_{1,13}=1.82, p=0.18$, group-depth interaction $F_{1,13}=0.53$ $\mathrm{p}=0.89$ ). Additionally, I calculated the proportion of sites with a BF of $16 \pm 1 \mathrm{kHz}$. Interestingly, the overall number of sites in the IC tuned to $16 \mathrm{kHz}$ increased $(11.81 \pm 1.8 \%$ vs $5.4 \pm 1.1 \%$ of sites, for exposed and control, respectively, $p=0.02$ ). This increased in the proportion of sites preferentially tuned to $16 \mathrm{kHz}$ seems to be specific effect, since the proportion of sites tuned to frequencies closed to $16 \mathrm{kHz}$ did not change $(13 \pm 1 \mathrm{kHz}: 11.23 \pm 1.7 \%$ vs $10.92 \pm 1.5 \%$, for exposed and control groups, respectively, $p>0.05$; and $19 \pm 1 \mathrm{kHz}: 10.7 \pm 1.8 \%$ vs $9.4 \pm 1.8 \%$, for exposed and control groups, respectively, $p>0.05$ ).

In summary, these results indicate that sound exposure modifies frequency representation by inducing an upward shift along the rostro-caudal axis of the high frequency laminas in the IC. Additionally, sound exposure specifically increases the proportion of sites tuned to its own frequency.

\subsubsection{Effect of days of sound exposure on collicular plasticity}

So far the experiments presented here have shown that sound exposure induces plastic changes in the IC of animals exposed to sounds in the Audiobox from 6-12 days. I was interested in determining at what point in time during sound exposure these changes can be detected (e.g. shift in frequency representation). For that purpose, I performed recordings from control and exposed animals to 16 $\mathrm{kHz}$ from day 1 until day 5 from exposure. For these experiments I used multi-electrode arrays with one shank. The control group included 68 recorded sites from 6 animals and the group included 51 recorded sites from 5 animals.

Figure 3.13 shows examples of raster plots at different depths from individual control (Figure 3.13 $A, B$ ), and exposed mice (Figure 3.13C,D). The strong evoked activity is evident in all the examples. It can be appreciated that the activity of the control mice is stronger than the exposed mice, particularly from 200-600 $\mu \mathrm{m}$. The average tuning curves reflected this difference (Figure 3.14). In 
the dorsal part (100-350 $\mu \mathrm{m})$ the tuning curves of both groups were more heterogeneous, evident by the multiple peaks, than the tuning curves of animals recorded from 6-12 days (Figure 3.4). I divided the recorded sites in zones as previously described and compared them between groups. There was a significant difference between groups (group effect: $F_{2,4}=47.96, p=5 \times 10^{-12}$; group-rank interaction: $F_{2,4}=4.4 \mathrm{p}=2 \times 10^{-7}$ ). With the exception of the putative dorsal cortex (group effect: $F_{1,23}=1.68, p=0.19$; group-frequency interaction: $\left.F_{1,23}=0.04, p=1\right)$, and the contiguous rank (200-350 $\mu \mathrm{m}$, group effect: $F_{1,23}=1.30, p=0.2$; group-frequency interaction: $F_{1,23}=1.06, p=0.37$ ) in the rest of the zones the activity of the control group was larger $\left(350-450 \mu \mathrm{m}\right.$, group effect: $F_{1,23}=67.13$, $\mathrm{p}=1 \times 10^{-15}$; group-frequency 

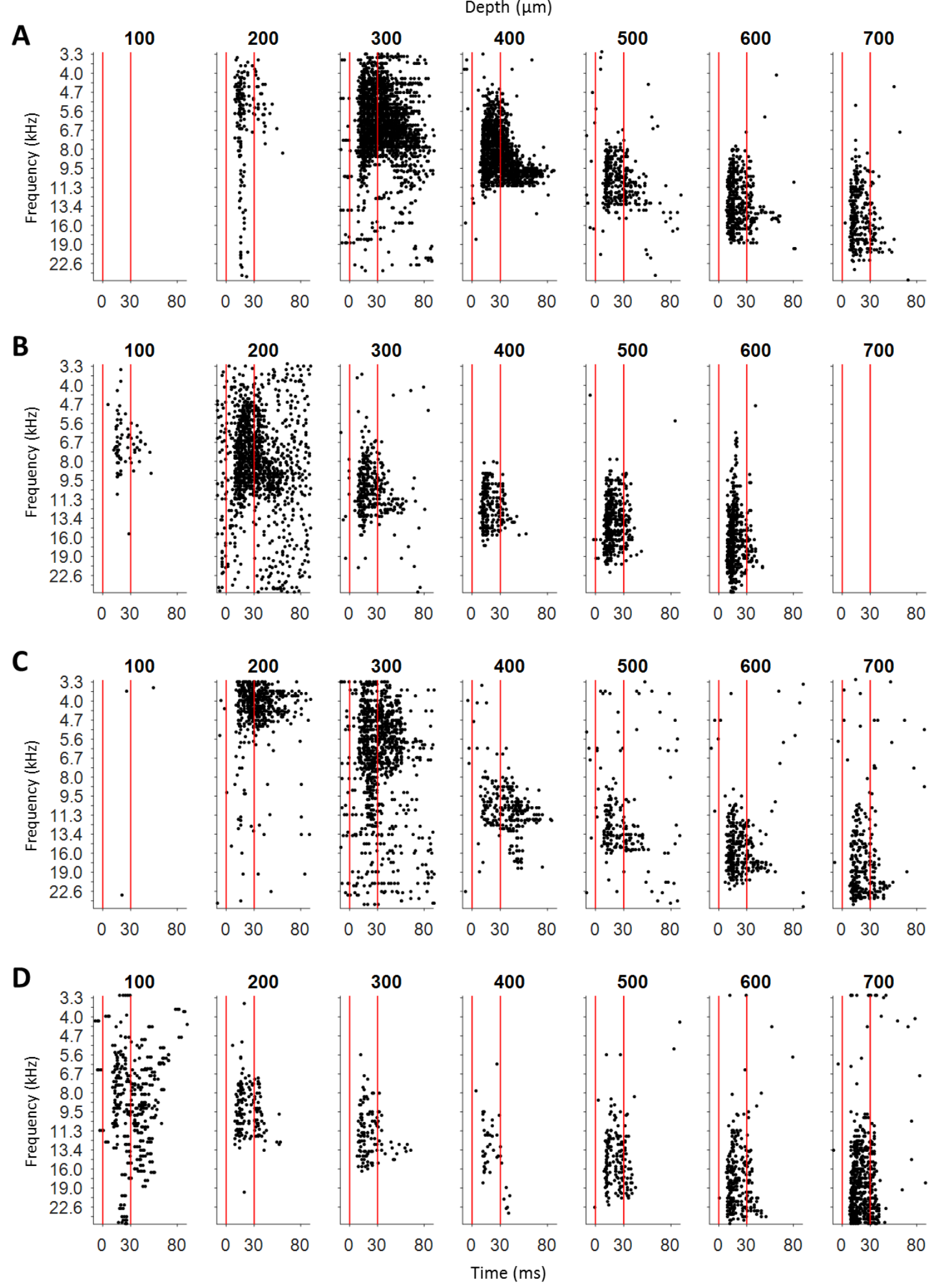

Figure 3.13: Evoked activity during the initial days of tone exposure. Representative examples of raster plots recorded from different depths from A-B, control; and C-D, relevant-exposed animals. Each dot represents a recorded spike. Five repetitions of $30 \mathrm{~ms}$ tones of different frequencies were played at $70 \mathrm{~dB}$ SPL. Vertical red lines indicate the duration of the tone. The recordings were obtained during 1-5 days after exposure to $16 \mathrm{kHz}$. 


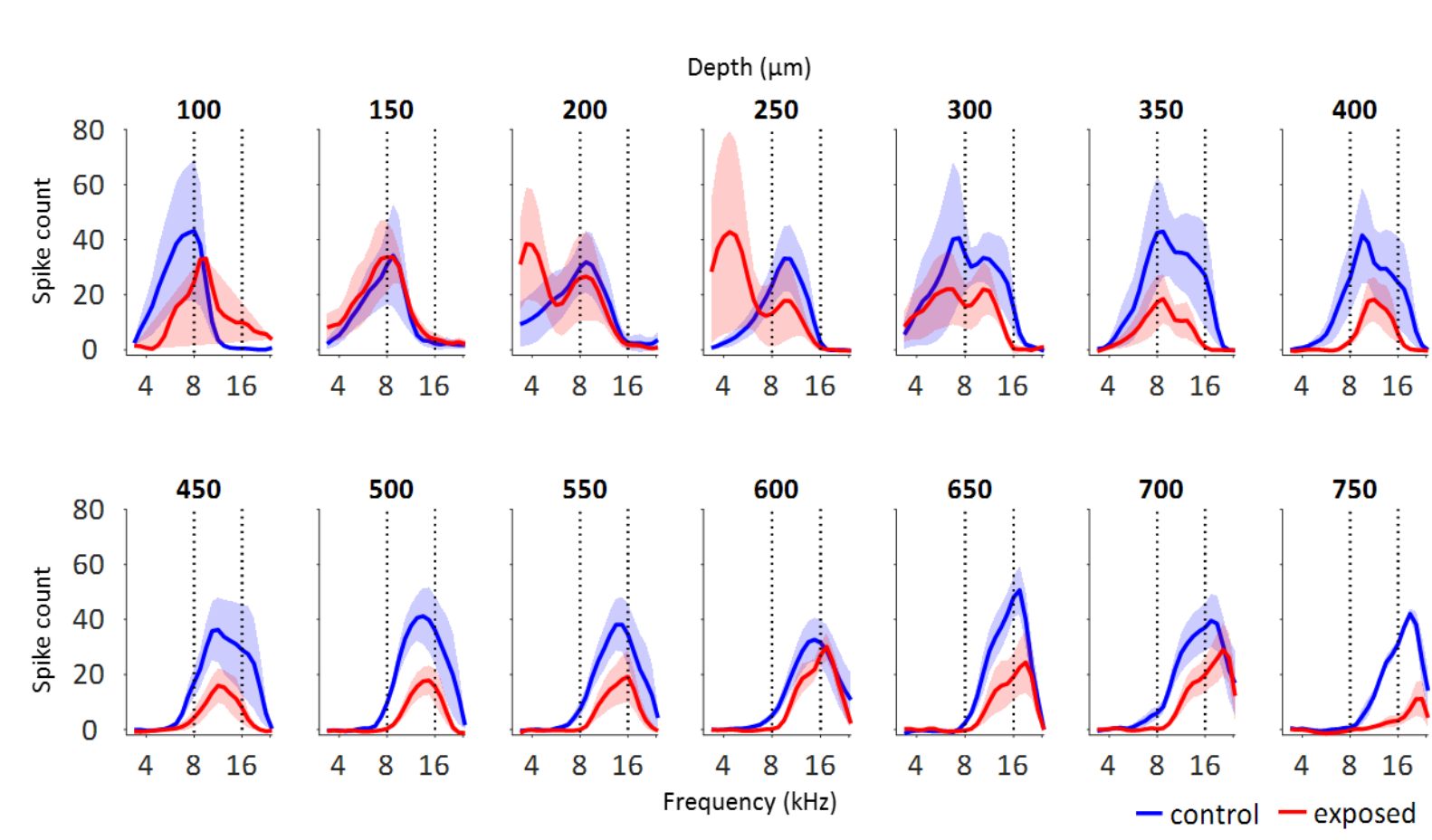

Figure 3.14: Effect during the initial days of tone exposure on the spectral tuning of the IC. Average tuning curves at $70 \mathrm{~dB}$ for different depths in the IC for control (blue) and relevant (red) exposed groups. The tuning curves were obtained during 1 to 5 days of exposure to $16 \mathrm{kHz}$.

interaction: $F_{1,23}=1.62, p=0.03 ; 500-600 \mu m, F_{1,23}=61.3, p=1.7 \times 10^{-14}$; group-frequency interaction: $F_{1,23}=1.9, p=0.006 ; 650-750 \mu m, F_{1,23}=43.4, p=1.2 \times 10^{-10}$; group-frequency interaction: $F_{1,23}=2.41$, $p=0.003)$. These results indicate that during the early days of sound exposure the activity of the control group is higher than the exposed group.

I compared the tuning curves at different depths, of the control and exposed groups during the two time windows of sound exposure: the early phase, which included the first 5 days of exposure; and the late phase, which included the $6^{\text {th }}$ to $12^{\text {th }}$ day of sound exposure. The statistical analysis showed that the activity of the control group was higher than all the groups during both phases of exposure (group effect, $F_{1,13}=15.37, p=8 \times 10^{-5}$; group-depth interaction, $F_{1,13}=2.41, p=0.003$; group-phase interaction, $F_{1,13}=82.92, p=1 \times 10^{-19}$; group-depth-phase interaction, $F_{1,13}=6.1, p=1.62 \times 10^{-11}$; pair comparisons, $p=4 \times 10^{-8}$ control early vs control late; and $p=4 \times 10^{-34}$ control early vs exposed late). Interestingly, the activity of the exposed group slightly decreased from the early to the late phase but it was not significantly different $(p=0.06)$. Together these results indicate that the activity of both groups changes along time spent in the Audiobox, with the control group exhibiting the most dramatically affected. In conclusion both groups undergo plasticity in the IC, probably due to the enriched environment of the Audiobox. Next, I analyzed the frequency representation of control and 

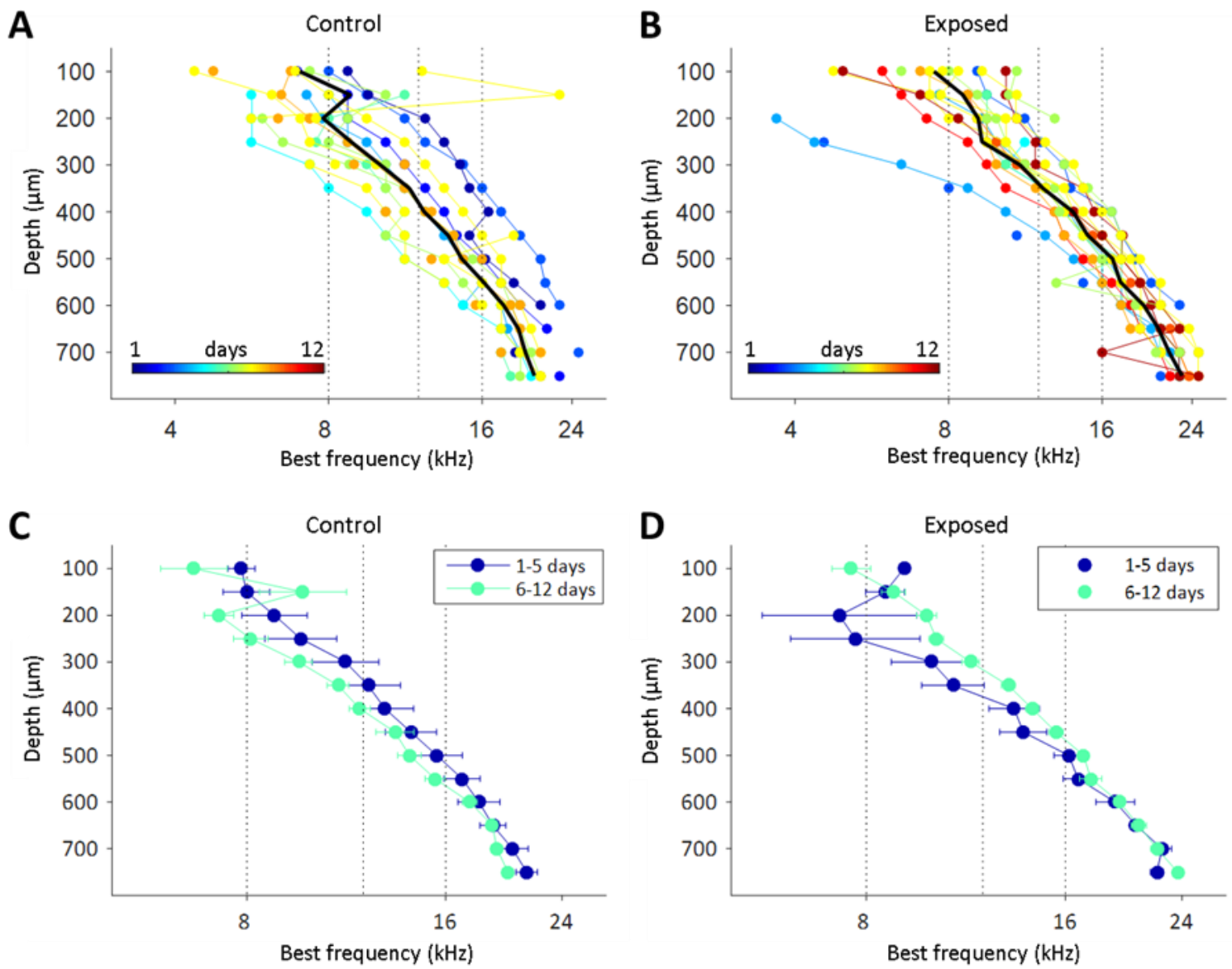

Figure 3.15: Effect of time of sound exposure on collicular tonotopy. Best frequencies of single animals recorded at different depths in the IC obtained at different days of sound exposure for $\mathbf{A}$, control group and $\mathbf{B}$, exposed group. The average is represented in black for each group. The frequency of the exposed tone was $16 \mathrm{kHz}$. The animals were grouped in two time windows: early (1-5 days) and late (6-12 days) according to the frequency distribution of the control animals. C, average best frequencies for control and $\mathbf{D}$, exposed group.

exposed animals during the early phase (1-5 days) of exposure. Ideally, I would expect that for example, frequency representation in exposed and control animals would be similar during the first day of exposure, and would gradually shift towards high frequencies for the exposed animals until it reached the values observed during the late phase (6-12 days) of exposure. To explore this idea, I plotted BF values of single animals recorded from day 1 to 12 of exposure. The single values of the control animals are shown in Figure 3.15A, and are color-coded according to the day of recording (colder color is close to day 1 and warmer color, to day 12). The black trace represents the group average. An interesting pattern can be observed, and is that control animals recorded within the first 5 days showed BF that were on or above the group average (5 out of 6 ), while the animals 
recorded from day 6 onwards showed BF below the mean average (7 out of 10). This indicates that in control

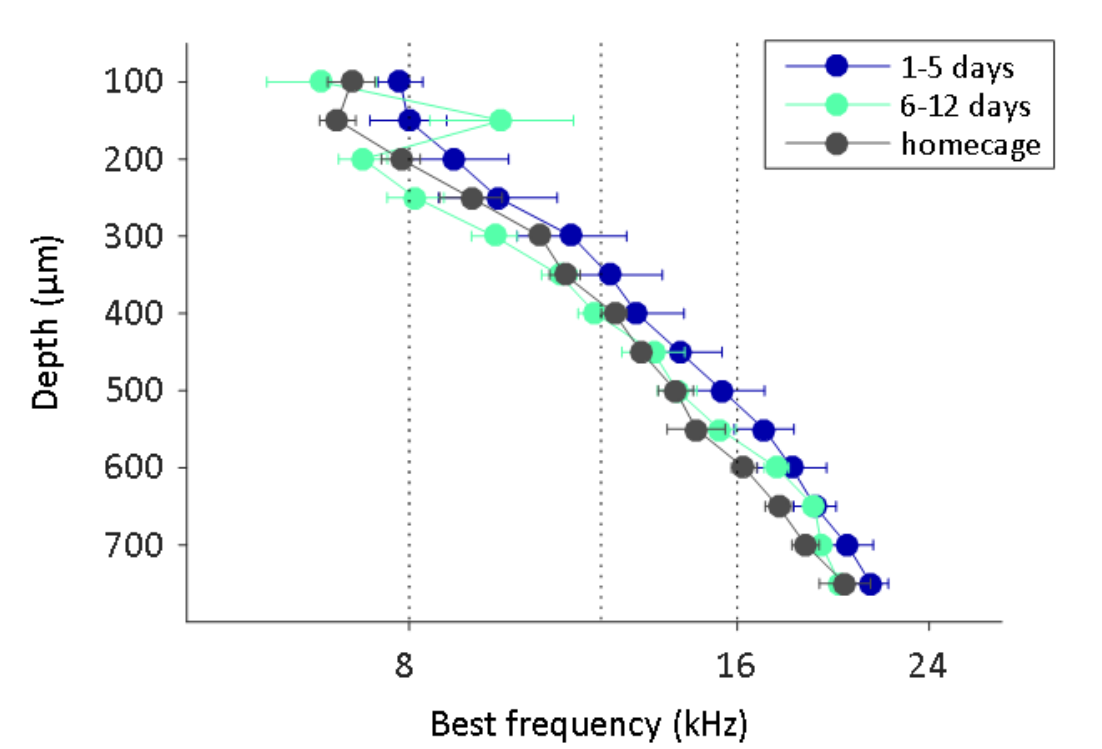

Figure 3.16: Effect of time of sound exposure on collicular tonotopy for the control and the homecage groups. Average best frequencies for the control group at different phases of exposure and for the homecage group. The control animals were grouped in two time windows: early (1-5 days) and late (6-12 days). The recordings of the homecage group were obtained from animals that lived in standard homecages similar amount of time as the animals from the late phase of sound exposure (7-14 days).

animals, there was a shift towards low frequencies with the exposure days. Clustering the control animals in groups according to this pattern (1-5 and 6-12 days) and averaging the BF in each depth (Figure $3.15 \mathrm{C}$ ) reflects the shift to low frequencies $\left(F_{2,13}=7, p=0.0012\right.$, group comparisons control early vs control late $p=0.016$ ). The same pattern was not observed in the exposed group (Figure 3.15B), where the values of $\mathrm{BF}$ of the animals recorded during the first 5 days of exposure were scattered on both sides of the mean. Nevertheless, averaging the clusters as in control animals revealed a shift towards high frequencies between the early and late days (Figure 3.15D; $F_{1,13}=5.19$, $\mathrm{p}=0.023)$. In conclusion, these results indicate that the differences in BF representation observed during 6-12 of exposure is the contribution of both groups that initially shift their BF in opposite directions.

It seems that changes in frequency representation in the IC are the norm rather than the exception. A question that immediately arose: what is the frequency representation of the animals before they are placed in the Audiobox? Are frequency representations of naïve animals more similar to the late phase of exposure or to the early phase? To answer this question, I performed recordings in 
the IC of animals that were kept in standard home cages in the same room where the Audiobox is located for the duration of the sound exposure experiments in the Audiobox (homecage group). Using multi-electrode arrays with one shank, 73 sites were recorded from 6 animals from the homecage group.

The average BF for each depth of the homecage group is shown in Figure 3.16 (black trace), plotted together with the average BF during 1-5 and 6-12 days of exposure of the control group (same as in $3.15 \mathrm{C})$. The average frequency distribution of the homecage group was more similar to the frequency distribution of the control animals during 6-12 days (Figure 3.16; green trace) than during the first 5 days of exposure (Figure 3.16 blue trace; $F_{2.13}=7, p=0.0012$, group comparisons: $p=0.79$, homecage vs late exposure; and $p=0.001$, homecage vs early exposure). These results indicate that placing the animals in the Audiobox, transiently alters frequency representation in the IC that returns to basal naïve levels after 6-12 days. Although surprising, these result are not illogical since the Audiobox is an enriched environment, also from the auditory perspective, different from that of the standard cage. For example, the noise of the sliding doors in the corner prior to access to water is also a behaviourally relevant sound. However, there are differences between this sound and the repetitive pips presented for the duration of an animal's visit. While the sound of the sliding door is triggered by a very specific behaviour of the animal and is associated with a visible movement of the door the animal just touched, the pips played for the duration of the visit can only be associated with the context in which the animal is but not with a particular behaviour or outcome.

\subsubsection{Effect of cortical inactivation in the IC}

In bats, it has been shown that the generation of collicular plasticity depends on direct cortical feedback through descending projections from the layer $V$ of the auditory cortex (Gao \& Suga 2000; Suga et al. 2002). To test whether the maintenance of the collicular plastic changes due to relevant sound exposure was dependent on cortical feedback, I performed simultaneous inactivation of the auditory cortex and recordings in the IC with multi-electrode arrays (1 shank, 16 electrodes, and penetration in the middle of the IC surface). For these experiments, the IC and ipsilateral AC were exposed. Saline solution was applied to the surface of the AC and the responses to sounds in the IC were recorded. After collecting the evoked responses in the absence of muscimol, a solution with muscimol was directly applied to the surface of the AC. Activity in the AC was monitored during the duration of the experiment through evoked local field potentials. Usually, after 20 minutes of muscimol application, evoked responses in the AC were no longer observed (Figure 3.17). Once cortical silencing was ensured, evoked responses in the IC were recorded again. I recorded IC 
responses from exposed and control animals from day 1 to 12 of exposure and analyzed in groups of early and late exposure as previously described. For the early phase (days 1-5) the control group included 62 sites recorded from 6 animals, for the exposed group it included 41 sites recorded from 5 exposed animals. During the late phase the

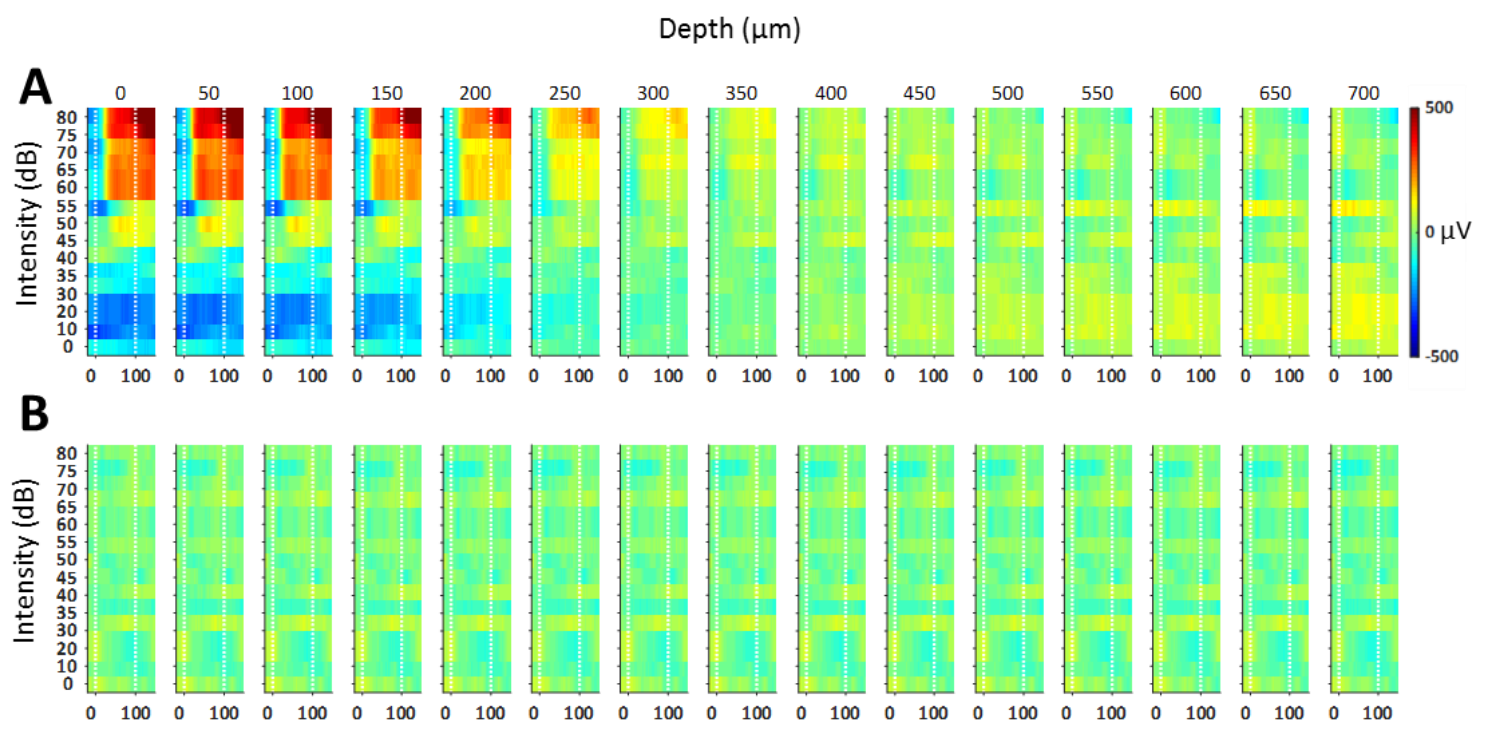

Figure 3.17: Inactivation of auditory cortex with muscimol. Representative color plots showing the simultaneous evoked LFP at different depths in the auditory cortex to stimulation with broadband noise at different sound intensities $\mathbf{A}$, before and $\mathbf{B}, 20$ minutes after muscimol application in the cortical surface. The vertical white dashed lines in each subplot represent the duration of the stimulus (100 ms).

control group included 63 sites recorded from 7 animals; and for the exposed group it included 64 sites recorded from 6 animals. The data collected from these animals prior to muscimol were a subset of the animals included in previous analysis. The analysis of the spectral tuning and frequency representation was made in the same way as in the previous analysis shown above.

During early phase of exposure (1-5 days), the average tuning curves are shown in the Figure 3.18. As mentioned before, with the exception of most dorsal areas, the average amplitude of the tuning curves of the control group were larger at multiple depths (350-750 $\mu \mathrm{m}$, Figure 3.18, continuous blue and red traces). Cortical inactivation induced larger evoked activity at multiple depths for both groups (400-700 $\mu \mathrm{m}$; Figure 3.18 dashed red and blue traces). However, the increase was larger in the exposed group $(6.43 \pm 0.55$ vs $9.88 \pm 0.98$, average increase in spike number per depth, for control and exposed, respectively) and eliminated the differences in the amplitude of the tuning curves observed before cortical inactivation. The initial statistical analysis showed that there was 
no significant group effect (group effect, $F_{1,13}=2.13, p=0.16$; group-muscimol interaction, $F_{1,13}=0.03$, $p=0.87)$, however it showed a significant interaction of muscimol and depth $\left(F_{1,13}=13.4, p=1.95 \times 10^{-}\right.$ ${ }^{18}$ ). Therefore, I decided to perform the analysis by particular zones of the recorded sites, to explore how cortical inactivation affected the particular areas of

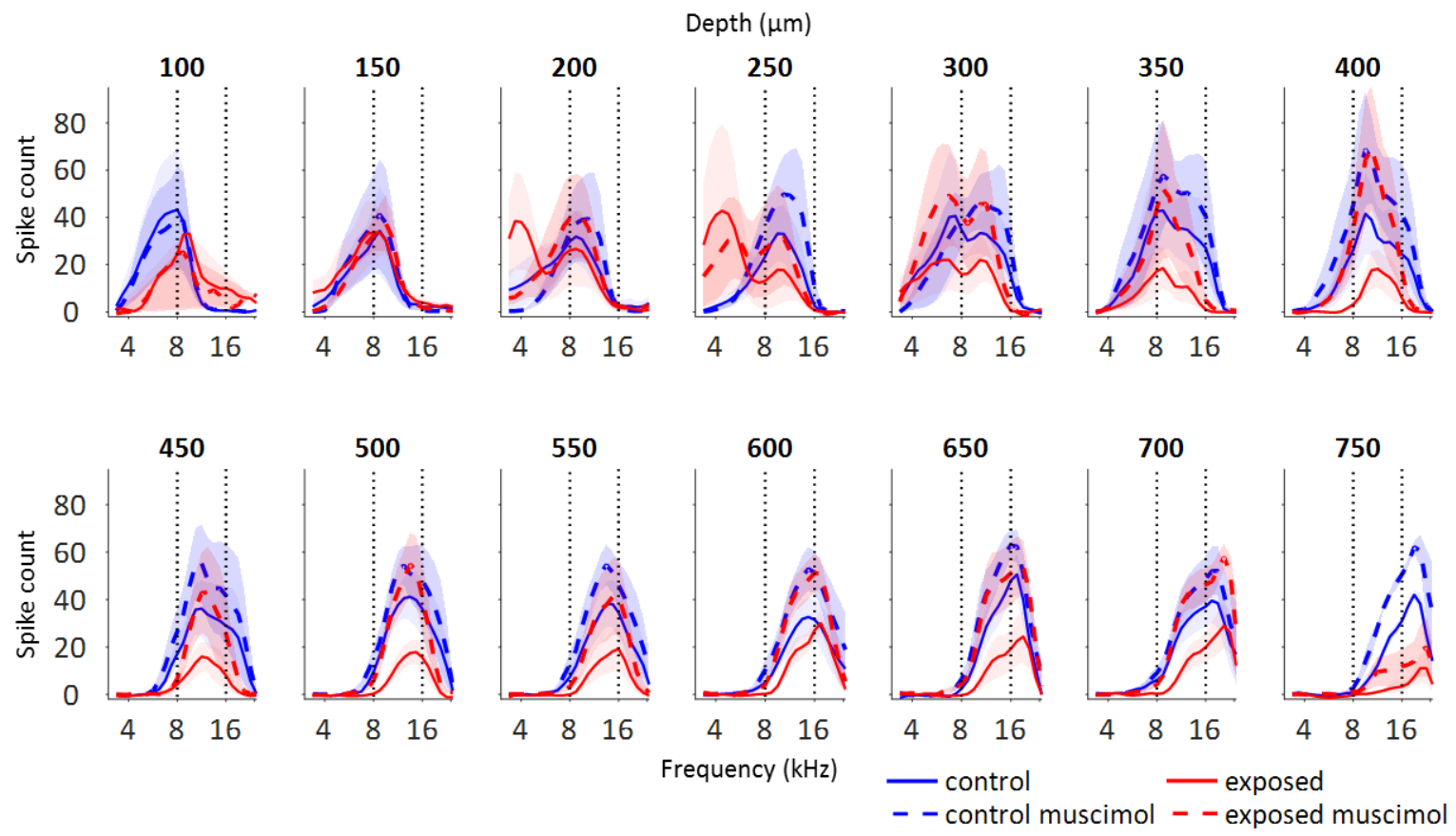

Figure 3.18: Effect of cortical inactivation during the early phase of tone exposure on the spectral tuning of the IC. Average tuning curves at $70 \mathrm{~dB}$ for different depths in the IC for control (blue) and relevant (red) exposed groups, before (continuous line) and after (dashed lines) cortical inactivation. The tuning curves were obtained during 1 to 5 days of exposure to $16 \mathrm{kHz}$.

the IC. Cortical inactivation had no effect on the putative dorsal cortex (100-150 $\mu \mathrm{m}$, group effect, $F_{1,23}=0.01, p=0.9$; muscimol effect $F_{1,23}=1.16, p=0.3$, group-muscimol interaction, $F_{1,23}=0.6, p=0.4$ ), and the contiguous dorsal area $\left(200-300 \mu m, F_{1,23}=0.5, p=0.4\right.$; muscimol effect $F_{1,23}=0.8, p=0.3$, group-muscimol interaction, $\left.\mathrm{F}_{1}=0.23, \mathrm{p}=0.63\right)$. In turn, cortical inactivation modified the responses in the ventral half of the IC in similar ways for both groups at 350-450 $\mu \mathrm{m}\left(\mathrm{F}_{1,23}=4.5, p=0.04\right.$; muscimol effect $F_{1,23}=23.23, p=5 \times 10^{-6}$, group-muscimol interaction, $F_{1}=1.12, p=0.29$ ), and with a higher impact on the exposed group in the area the area tuned to $16 \mathrm{kHz}$, where muscimol induced larger tuning curves in the exposed group $\left(500-600 \mu m, F_{1,23}=3.4, p=0.07\right.$; muscimol effect $F_{1,23}=104.9 .2, p=1.2 \times 10^{-10}$, group-muscimol interaction, $F_{1,23}=7.8, p=0.009 ; 650-750 \mu m, F_{1,23}=3$, $p=0.09$; muscimol effect $F_{1,23}=94.3, p=4 \times 10^{-8}$, group-muscimol interaction, $F_{1}=2.5, p=0.12$ ).

During the late phase of exposure (6-12 days), the amplitude of the tuning curves of the exposed animals was larger than the control animals and the peaks shifted towards high frequencies at 
multiple depths (Figure 3.19; 200-600 $\mu \mathrm{m}$ ). The amplitude was lower than control animals at depths above $600 \mu \mathrm{m}$, when the BF was above $16 \mathrm{kHz}$ (Figure 3.19, same code as previous figure). The initial statistical analysis revealed a significant effect of group, and significant interaction of muscimol and

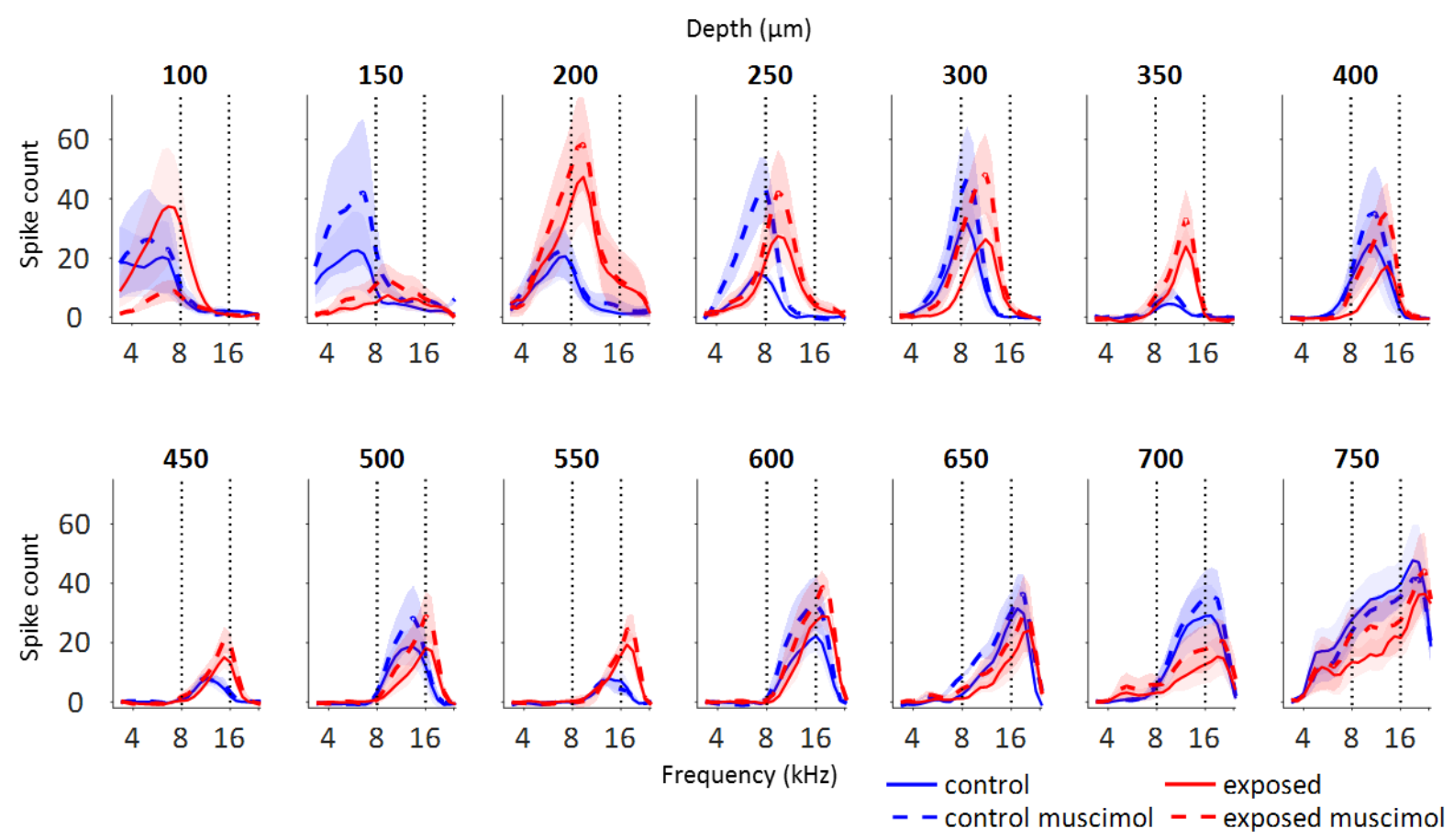

Figure 3.19: Effect of cortical inactivation during the late phase of tone exposure on the spectral tuning of the IC. Average tuning curves at $70 \mathrm{~dB}$ for different depths in the IC for control (blue) and relevant (red) exposed groups, before (continuous line) and after (dashed lines) cortical inactivation. The tuning curves were obtained during 6 to 12 days of exposure to $16 \mathrm{kHz}$.

depth (group effect, $F_{1,13}=4.36, p=0.04 ;$ muscimol-depth interaction, $F_{1,13}=4.57, p=1.7 \times 10^{-6}$ ). The analysis by zones, revealed that with the exception of the putative dorsal cortex (100-150 $\mu \mathrm{m}$, group effect, $F_{1,23}=0.6, p=0.4$; muscimol effect $F_{1,23}=0.04 p=0.8$, group-muscimol interaction, $F_{1,23}=2.7$, $\mathrm{p}=0.1)$, cortical inactivation modulated the evoked activity in the IC $(200-600 \mu \mathrm{m})$ of control and exposed group in equal magnitudes $\left(200-300 \mu m, F_{1,23}=2.09, p=0.15\right.$; muscimol effect $F_{1,23}=13$, $p=0.0011$, group-muscimol interaction, $F_{1}=0.3, p=0.5 ; 350-450 \mu m, F_{1,23}=0.07, p=0.78$; muscimol effect $F_{1,23}=8.7, p=0.0066$, group-muscimol interaction, $F_{1}=1.6, p=0.2 ; 500-600 \mu m, F_{1,23}=0.1, p=0.7$; muscimol effect $F_{1,23}=6.09, p=0.02$, group-muscimol interaction, $\left.F_{1}=0.01, p=0.9\right)$. The increase in the amplitude only eliminated the differences in evoked activity between groups before cortical inactivation in the most ventral area $(600-750 \mu \mathrm{m})$, but not the differences in the frequency of the peak $\left(F_{1,23}=1.57, p=0.2\right.$; muscimol effect $F_{1,23}=4.6, p=0.04$, group-muscimol interaction, $F_{1}=1.46$, $\mathrm{p}=0.2)$. 
To further characterize the effect in frequency representation after cortical inactivation, the average $\mathrm{BF}$ at different depths between control and exposed animals were compared before and after cortical inactivation (Figure 3.20). During the early phase the distribution of BFs between control and relevant exposed animals was similar (Figure 3.21A; $F_{1,13}=0.0537, p=0.8219$ ). Cortical inactivation did

A

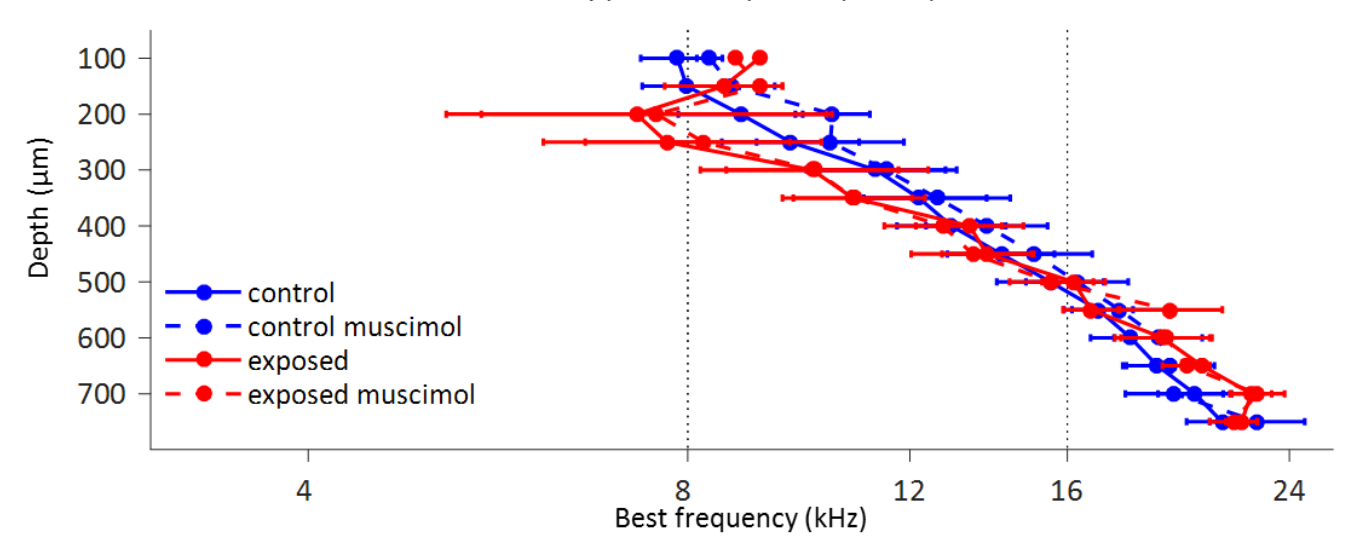

B

B Late phase of exposure $(16 \mathrm{kHz})$

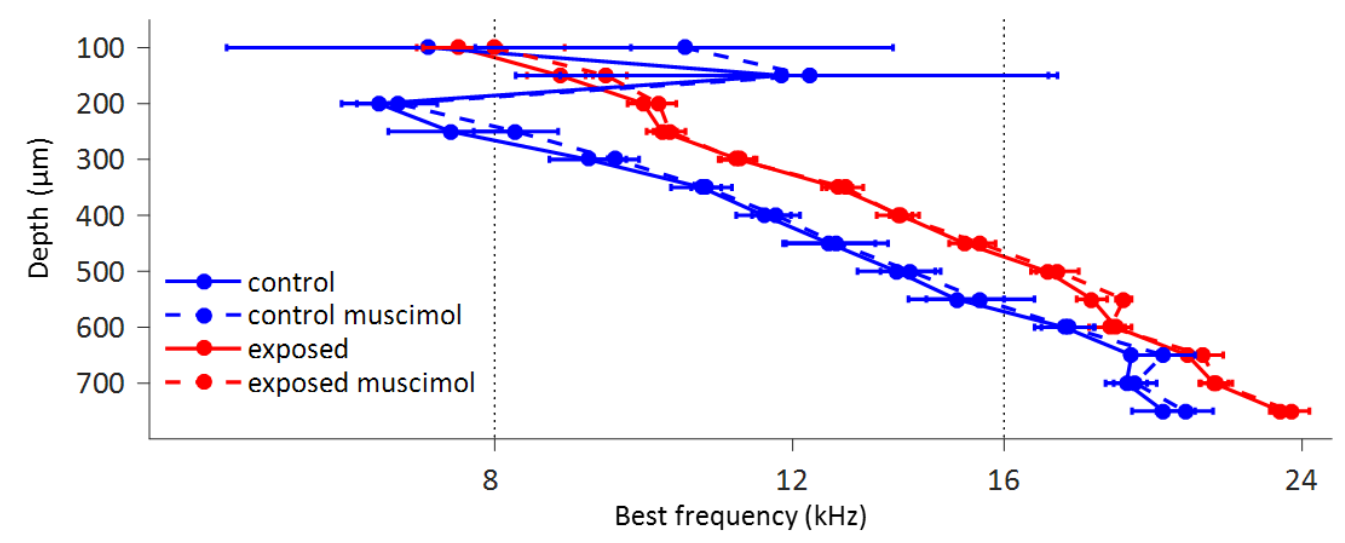

Figure 3.20: Effect of cortical inactivation on collicular tonotopy. Average best frequencies for different depths in the IC obtained during A, the early phase ( 1 to 5 days) and $\mathbf{B}$, the late phase (6 to 12 days) of tone exposure to $16 \mathrm{kHz}$ for control (blue) and relevant (red) exposed groups, before (continuous line) and after (dashed lines) cortical inactivation.

not change BFs (group-muscimol interaction $\mathrm{F}_{1,13}=0.04 \mathrm{p}=0.8$ ) at any tested depth (group-depthmuscimol interaction $\mathrm{F}_{1,13}=1.33, \mathrm{p}=0.21$ ). During the late phase, in the subset of exposed animals used in this experiments, there was a consistent shift in BFs along different depths of the IC (consistent with the effect of the whole group previously shown) already evident in the peak of the tuning curves (Figure 3.20B; $F_{1,13}=15.7, p<0.01$; group-depth interaction $F_{1,13}=3.47, p<0.001$ ). 
Cortical inactivation did not alter this difference (group-depth-muscimol interaction $F_{1,13}=0.65$, $\mathrm{p}=0.79)$.

In summary, cortical inactivation affected the spectral tuning of both control and exposed groups in the two different time windows of sound exposure. The main effect was an increase in the amplitude of the tuning curves, mainly in the ventral half of the IC. During the early phase of sound exposure, the effect was larger on the exposed group in the ventral area and eliminated the previous differences in multiple depths. In the late phase of sound exposure, the increase in the amplitude of

\subsubsection{Analysis of spectral tuning in the lateral cortex of the IC}

The lateral cortex (LC) of the IC is multisensory nucleus that receives projections from different brain areas (Oliver 2005). Compared to the IC, the LC has broader spectral tuning (Syka et al. 2000) and the tonotopic organization in the LC of the mouse has not being well characterized. To assess whether sound exposure induces changes in the LC, I performed recordings in the LC during the early and late phase of sound exposure to $8 \mathrm{kHz}$. In a set of mice, after recordings in the central IC were made, the single electrode was moved $450-500 \mu \mathrm{m}$ lateral and recordings were made at different depths of the LC. The control group included 11 recorded sites from 5 animals in the early phase and 12 recorded sites from 4 animals in the late phase. The exposed group included 7 recorded sites from 3 animals in the early phase and 13 recorded sites from 5 animals in the late phase. The recordings made in the lateral portion of the IC confirmed the broader tuning previously reported and was easily distinguished from those in the central IC (Figure 3.21).

Figure 3.21 represents example raster plots of recordings performed in the LC of individual mice. Compared to the recordings in the central IC (Figure 3.21), the evoked responses in the LC were widely tuned and showed no evident tonotopy with increasing depth, in agreement with previous reports (Syka et al. 2000). The evoked responses of the control mouse during the early phase of exposure (Figure 3.21A; 1-5 days) were larger than the evoked responses of the control mouse during the late phase of exposure (Figure 3.21B; 6-12 days). Although to a lesser extent, this pattern was similar for the exposed animals (Figure 3.21 C and D for early and late exposure respectively). The statistical analysis revealed that, indeed, the activity of the control mice decreased from the early to the late phase of exposure, while the exposed animal relatively stable (group effect $F_{1}=0.39$, $p=0.53$; exposure phase effect $F_{1}=15.04, p=0.0001$; group-phase interaction, $F_{1}=3.6, p=0.057$, pair comparisons, $p=0.0003$ control early vs control late; $p=0.003$ exposed early vs control late; and $p=0.9$ exposed early vs exposed late).These results in activity were similar to the decrease in evoked 
activity seen in the central IC, indicating an overall excitability state in the IC during the first days of living in the Audiobox.

Next, I compared the different groups during the two, early and late, exposure phases. During the early phase, the exposed group had larger evoked activity in one out of three locations measured (Figure 3.21C $200 \mu \mathrm{m}$ ). The average tuning curves also reflected this difference (Figure 3.22A; 200 $\mu \mathrm{m} \mathrm{F}_{1,23}=14.17, p=0.0003$, group-frequency interaction $\mathrm{F}_{1,23}=0.2, p=0.9 ; 400 \mu \mathrm{m} \mathrm{F}_{1,23}=0.7, p=0.4$, the tuning curves caused by cortical inactivation was smaller and equally affected both groups. Interestingly, it did not modify the BFs neither in the early nor late phases, indicating that cortical feedback is not required to maintain the collicular plasticity.
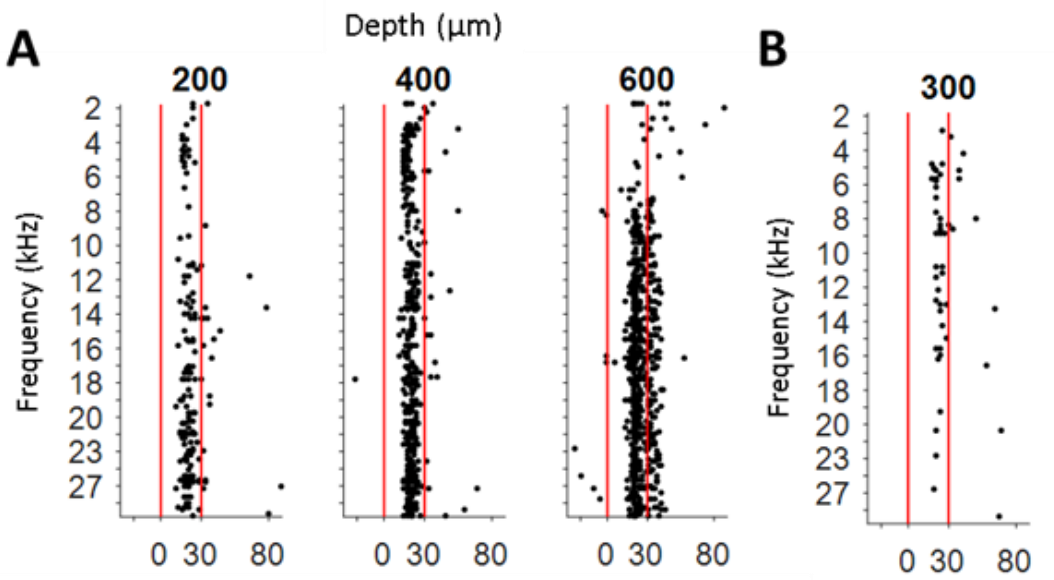

Depth $(\mu \mathrm{m})$

C
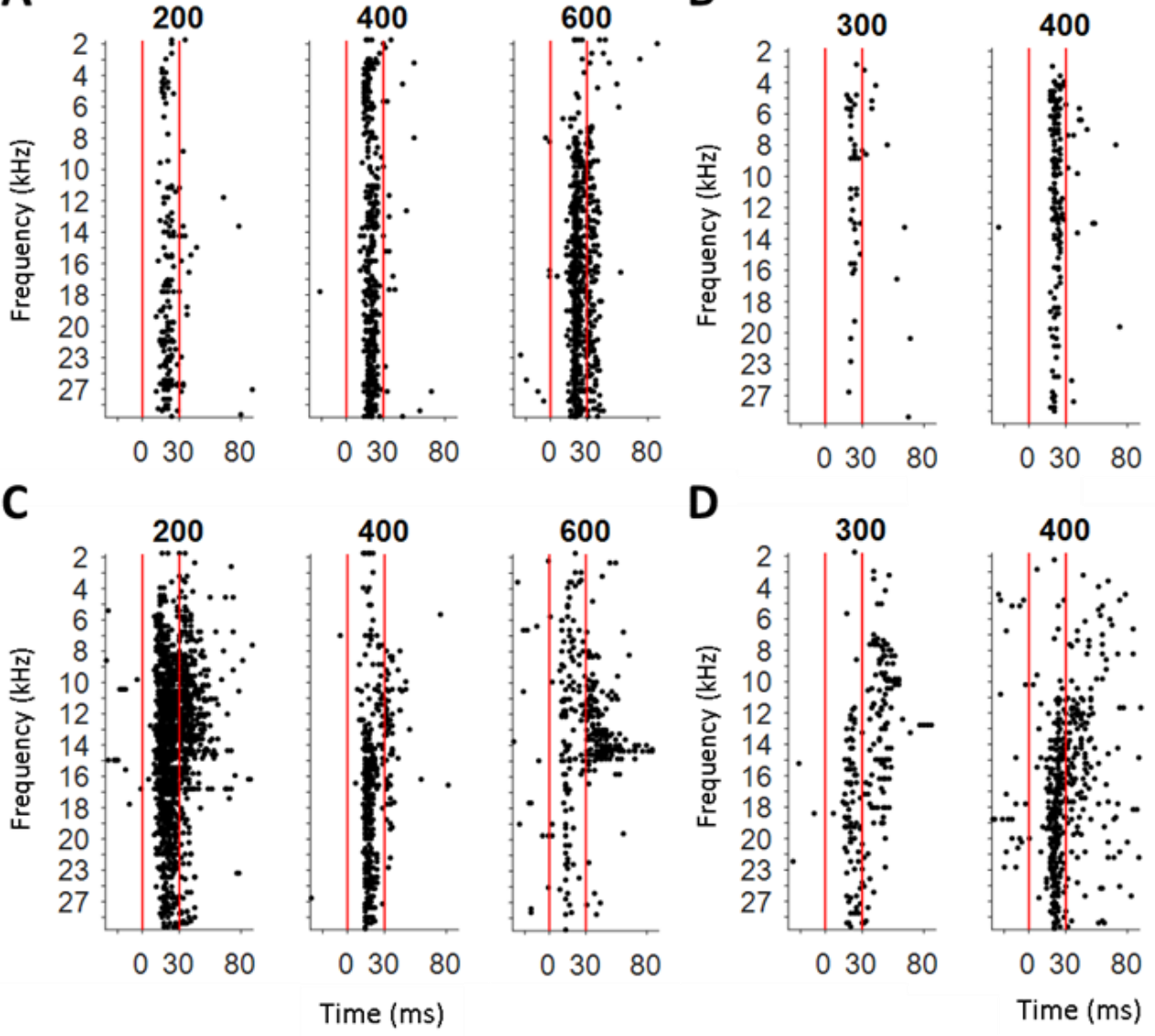

600
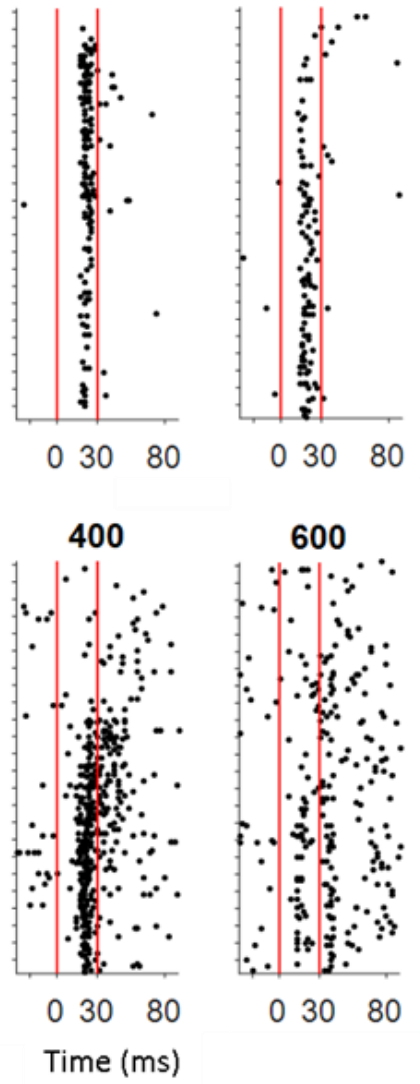

Figure 3.21: Evoked activity in the lateral cortex of the IC during the early and late phases of tone exposure. Representative examples of raster plots recorded from the lateral cortex at different depths from A, one control and; C, one exposed animal during the early phase (1-5 days) and; $\mathbf{B}$, one control and; $\mathbf{D}$, one exposed animal during the late phase (6-12 days) of tone exposure to 8 $\mathrm{kHz}$. Each dot represents a recorded spike. Five repetitions of $30 \mathrm{~ms}$ tones of different frequencies were played at $70 \mathrm{~dB}$ SPL. Vertical red lines indicate the duration of the tone. 
group- frequency interaction $F_{1,23}=0.2, p=0.9 ; 600 \mu m F_{1,23}=13.92, p=0.0003$, group-frequency interaction $\mathrm{F}_{1,23}=0.2, \mathrm{p}=0.9$ ).

In the late phase, the evoked responses (Figure 3.21B,D) and the amplitude of the tuning curves were also higher for the exposed animals in two out of three recording locations (Figure 3.22B) (300 $\mu \mathrm{m} \mathrm{F}_{1,23}=9.42, p=0.0025$, group-frequency interaction $\mathrm{F}_{1,23}=1.92, p=0.01 ; 400 \mu \mathrm{m} \mathrm{F}_{1,23}=49.28$, $p<0.001$, group-frequency interaction $\left.F_{1,23}=2.75, p<0.001\right)$ and lower at $500\left(F_{1,23}=32.66, p<0.0001\right.$, group-frequency interaction $\left.F_{1,23}=1.02, p=0.4\right)$ and $600 \mu m\left(F_{1,23}=13.95, p=0.0003\right.$, group-frequency interaction $F_{1,23}=0.42, p=0.9$ ). Interestingly, the tuning curves of the exposed animals were sharper than control animals, mainly due to a reduction in the responses frequencies below $8 \mathrm{kHz}$ (Figure 3.22B).
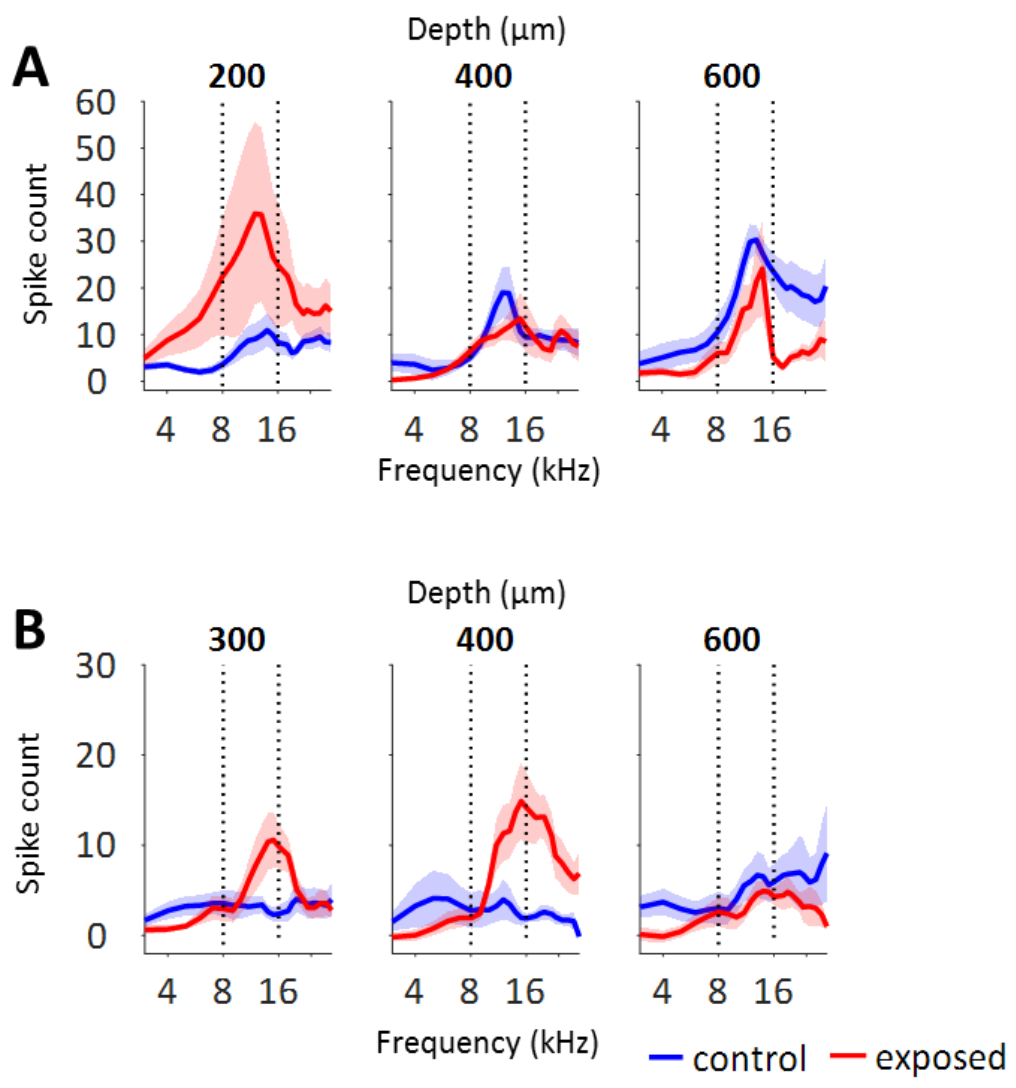

Figure 3.22: Effect during the early and late phases of tone exposure on the spectral tuning of the lateral cortex of the IC. Average tuning curves at $70 \mathrm{~dB}$ for different depths in the lateral cortex of the IC for control (blue) and relevant (red) exposed groups during $\mathbf{A}$, the early phase (1-5 days) and B, the late phase (6-12 days) of tone exposure to $8 \mathrm{kHz}$.

In summary, the activity in the LC decreased from early to late phase of exposure in both groups, suggesting an increase in overall excitability in the IC during the first days of living in the Audiobox. 
However, sound exposure induced larger tuning curves during the early phase of exposure that was maintained and extended to other locations of the LC in the late phase.

\subsection{Molecular analysis}

\subsubsection{Gene expression analysis}

To investigate whether the observed electrophysiological changes in animals exposed to $16 \mathrm{kHz}$ were accompanied with molecular changes in the IC or AC, I measured the expression of different genes involved in synaptic transmission and neuronal plasticity that have been reported to change its expression levels upon sound exposure, acoustic learning paradigms or enriched environment (Ortinski et al. 2004; Dong et al. 2010; Holt et al. 2005; Browne et al. 2012; Marianowski et al. 2000; Tan et al. 2007; Mainardi et al. 2010). The genes analyzed were grouped in the following categories: immediate early genes, genes associated to inhibitory transmission, excitatory transmission, or

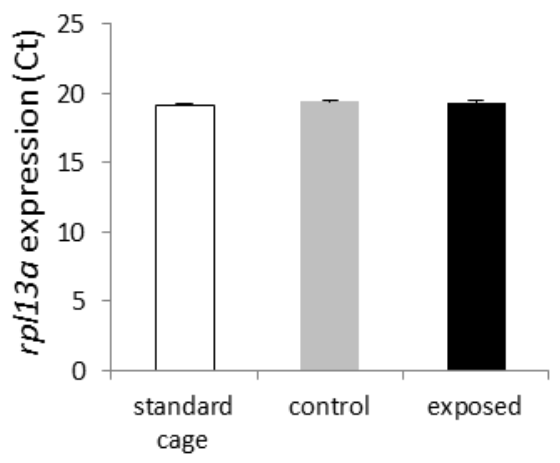

Figure 3.23: Gene expression in the IC of the housekeeping gene rpl13a. Gene expression was measured by RT-qPCR. Average Ct values of standard cage, control and exposed groups. Samples were collected at 7 days of sound exposure to $16 \mathrm{kHz}$.

neuronal plasticity, and neurotrophic factors (Table 3.8). To test if the environment in the Audiobox itself induced changes, I also measured gene expression in age-matched mice that were kept in standard home cages.

I used the gene of the Ribosomal protein L13a $(r p / 13 a)$ as a reference gene, since it has been reported as the best candidate gene for brain gene expression analysis (Gubern et al. 2009). The relative expression of Rpl13a showed no change between the three groups tested (Figure 3.23; $F_{2,17}=0.8, p=0.47, n=7,8$ and 5 for exposed, control and homecage groups, respectively).

There were no differences in the expression of the immediate early gene arc (Figure 3.24A). The detailed statistical values for all the genes analyzed are provided in Table 3.8. The expression of 
cfos showed a tendency to be downregulated in the control and exposed groups (Figure 3.24B). There was a robust increase in the expression of the transcription factor creb for the groups that lived in the Audiobox, regardless of sound exposure (Figure 3.24C; $p<0.001$ for control and exposed vs standard cage.

The relative expression of the genes involved in inhibitory transmission remained unchanged for the different subunits of the GABAa receptor (Figure 3.25A-D) and gad1 (Figure 3.25E). There was a slight reduction in vgat expression for the control and exposed groups (Figure 3.25F; $p>0.05$ ). In the group of genes related to excitatory transmission, compared to the standard cage animals the expression of gria1 and gria2 were reduced in the control group (Figure 3.26D,E; $p=0.004$ and $p=0.04$, respectively). It is important to note that the exposed group showed a tendency towards decrease for gria1 $(p=0.06)$. There were no changes in the expression in the rest of the genes in this category (Figure 3.26A-C,F). 

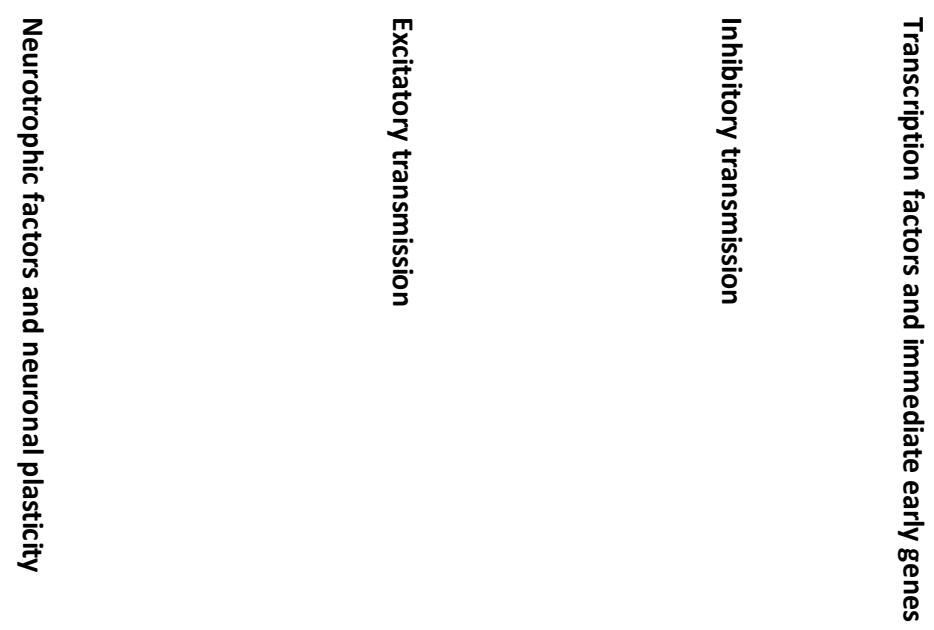

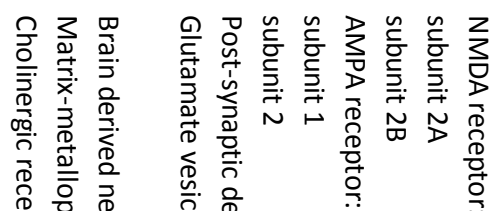
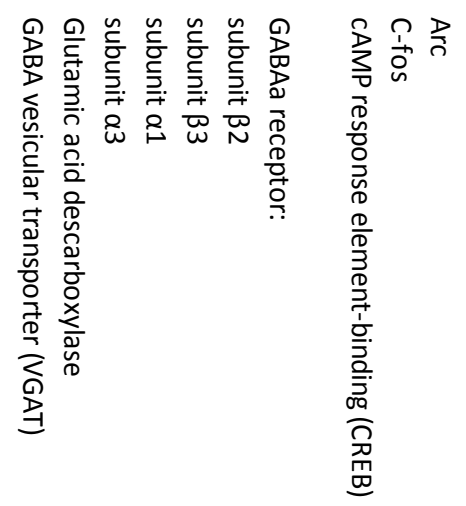

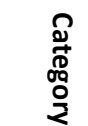

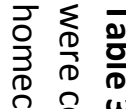

ชิ

한.

织离署

물.

골

政

ฏ

要

ำ

3 일

잉

तิ

官 它

응 조N

글.

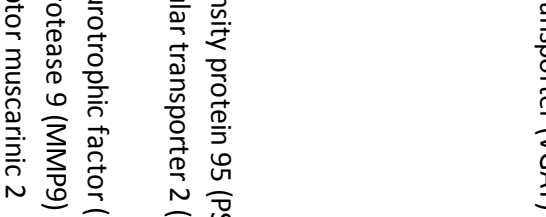

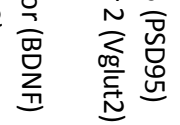

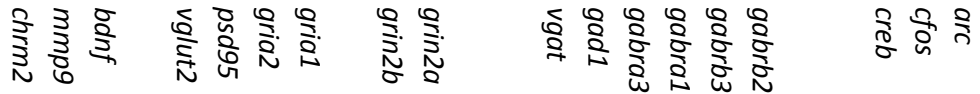

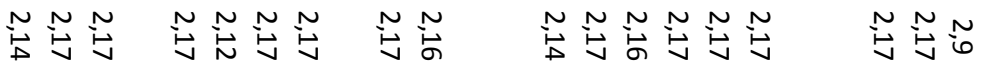

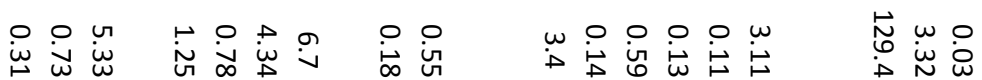

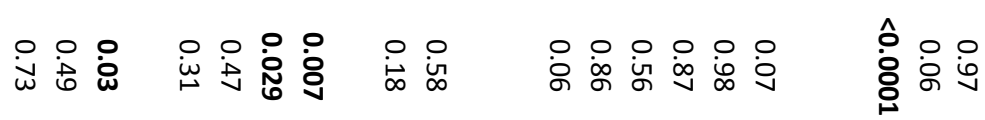

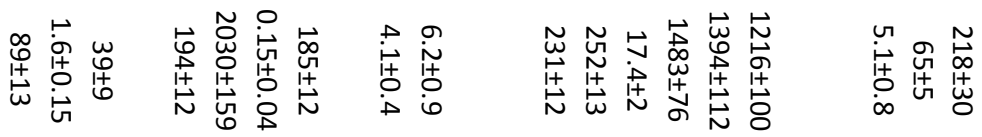

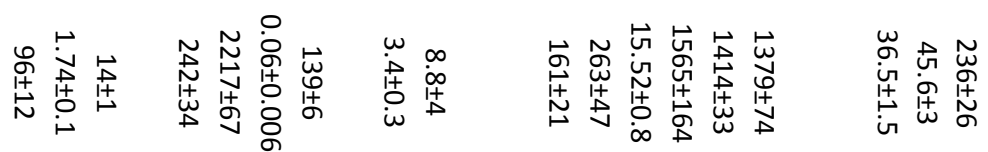

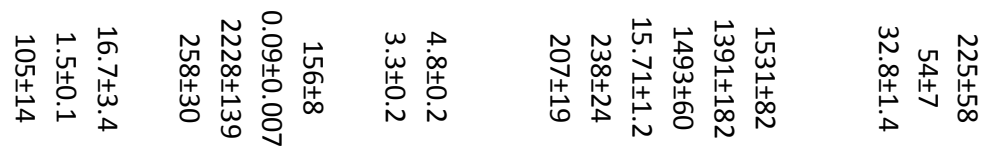


A

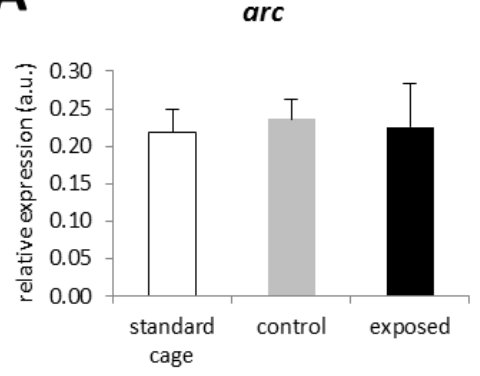

B

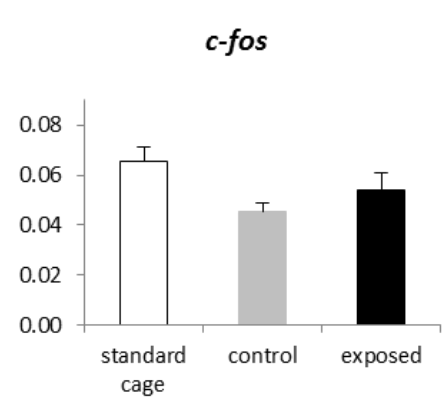

C

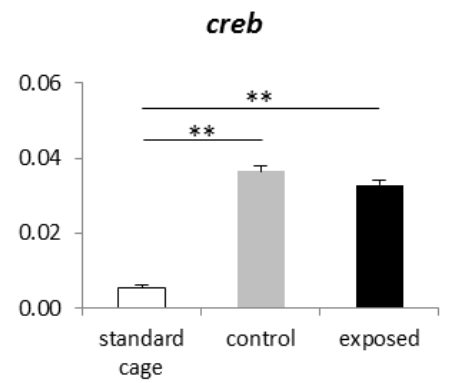

Figure 3.24: Effect of tone exposure on the collicular gene expression of immediate early genes. Gene expression was measured by RT-qPCR. Relative expression of A, arc; B, cfos and C, creb. Samples were collected at 7 days of sound exposure to $16 \mathrm{kHz}$. ${ }^{* *} \mathrm{p}<0.001$.

A

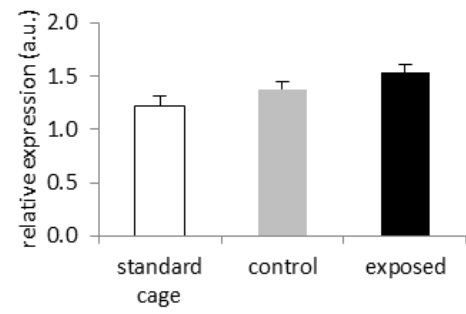

D

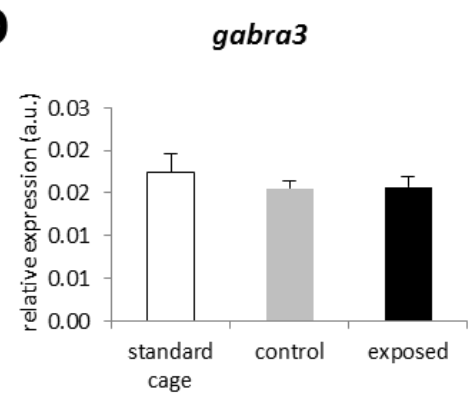

B

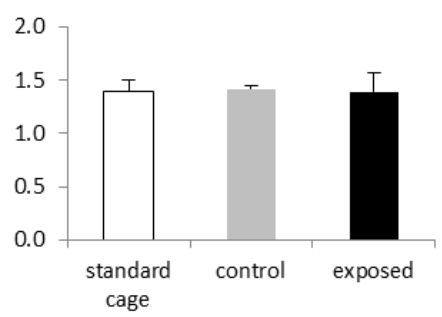

$\mathbf{E}$

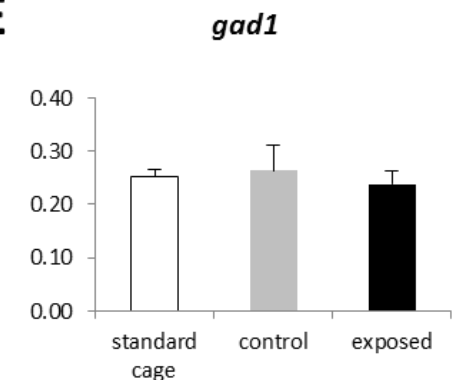

C

gabra1

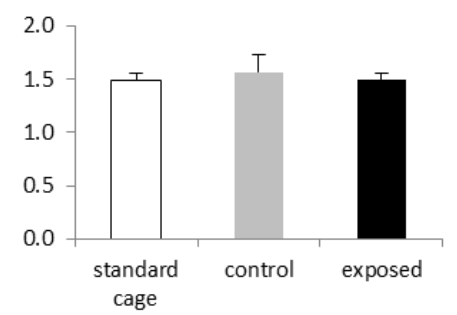

$\mathbf{F}$

vgat

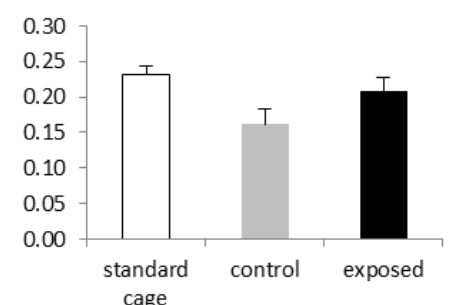

Figure 3.25: Effect of tone exposure on the collicular gene expression of genes involved in inhibitory transmission. Gene expression was measured by RT-qPCR. Relative expression of $A$, subunit $\beta 2$ of the GABAa receptor, gabrb2; B, subunit $\beta 3$ of the GABAa receptor, gabrb3; C, subunit $\alpha 1$ of the GABAa receptor, gabra1; D, subunit $\alpha 3$ of the GABAa receptor, gabra3; E, glutamic acid descarboxylase, gad1; F, GABA vesicular transporter, vgat. Samples were collected at 7 days of sound exposure to $16 \mathrm{kHz}$.

The expression of bdnf in the control group was significantly decreased compared to the standard cage group (Figure $3.27 \mathrm{~A} ; \mathrm{p}=0.04$ ). The exposed group also showed a tendency in the same direction ( $p>0.05$ ). There were no differences in the expression of mmp9 and chrm2 (Figure 3.27B,C). 
A

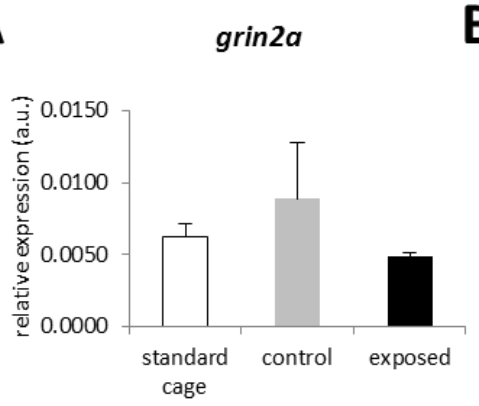

D

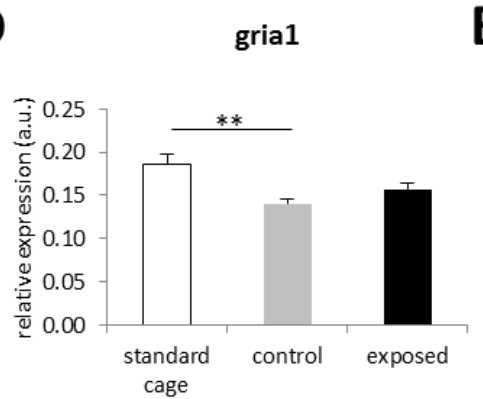

B

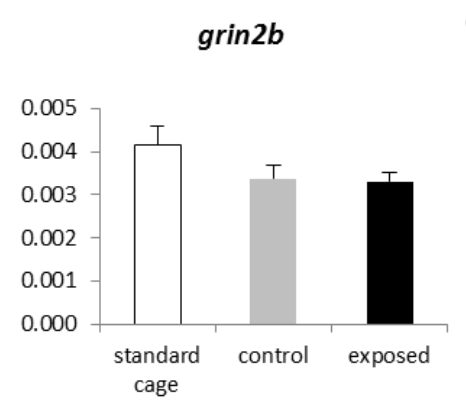

E

gria2

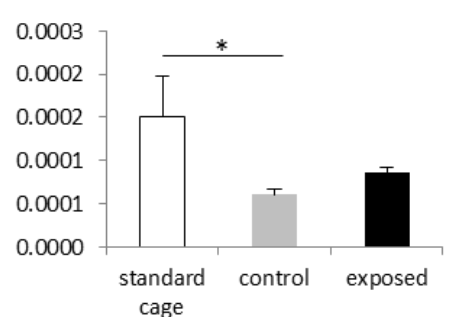

C psd95

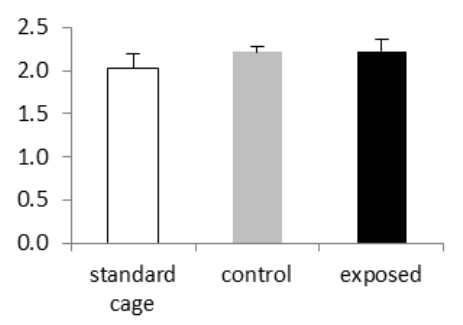

$\mathbf{F}$

vglut2

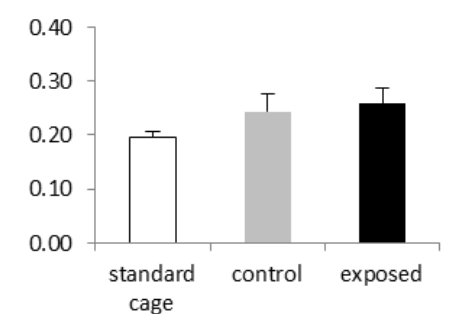

Figure 3.26: Effect of tone exposure on the collicular gene expression of genes involved in excitatory transmission. Gene expression was measured by RT-qPCR. Relative expression of $A$, Subunit $2 \mathrm{~A}$ of the glutamate NMDA receptor, grin2a; $\mathrm{B}$, subunit $2 \mathrm{~B}$ of the glutamate NMDA receptor, grin 2b; C, Post-synaptic density protein 95, psd95; D, glutamate receptor AMPA 1, gria1; E, glutamate receptor AMPA 2, gria2; F, glutamate vesicular transporter 2, vglut2. Samples were collected at 7 days of sound exposure to $16 \mathrm{kHz}$. ${ }^{*} p<0.05,{ }^{* *} p<0.001$.

A

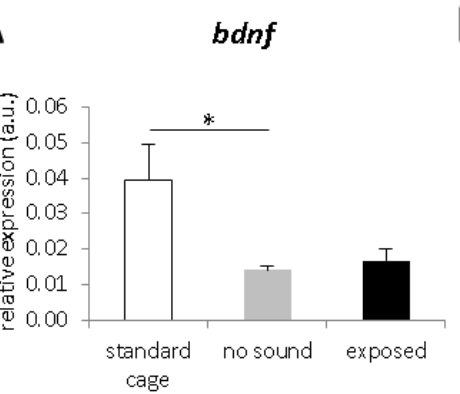

B

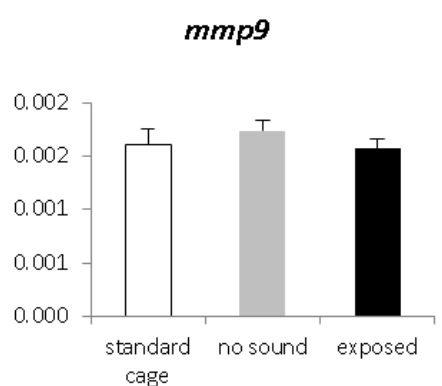

cage

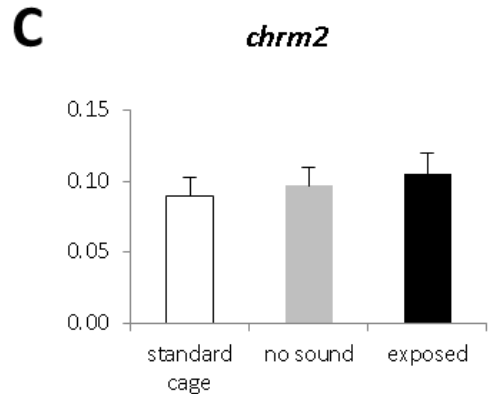

Figure 3.27: Effect of tone exposure on the collicular gene expression of genes involved in neuronal plasticity. Gene expression was measured by RT-qPCR. Relative expression of $\mathbf{A}$, brain derived neurotrophic factor, bdnf; $\mathbf{B}$, matrix-metalloprotease 9, mmp9; C, cholinergic receptor muscarinic 2, chrm2. Samples were collected at 7 days of sound exposure to $16 \mathrm{kHz}$. ${ }^{*} \mathrm{p}<0.05$.

Interestingly, the ratio between the expressions of vglut2/vgat showed a significant increase for control and exposed groups (Figure 3.28; $F_{2,14}=12.78 p<0.001, p<0.01$ standard cage vs control and exposed groups). 


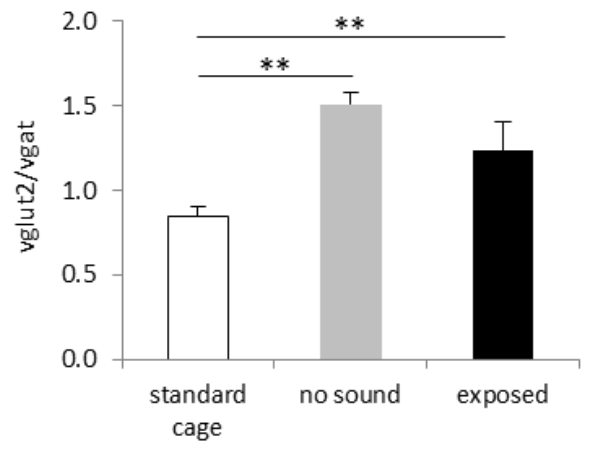

Figure 3.28: Effect of tone exposure on the collicular vglut2/vgat expression ratio. Gene expression was measured by RT-qPCR. Samples were collected at 7 days of sound exposure to 16 kHz. ${ }^{* *} \mathrm{p}<0.001$.

A

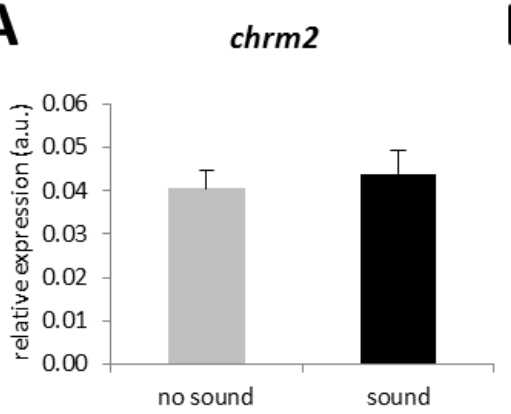

C

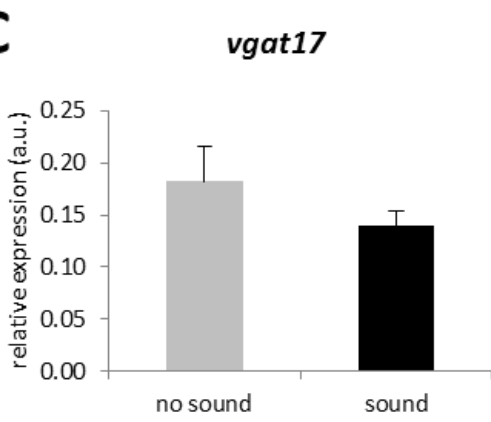

B

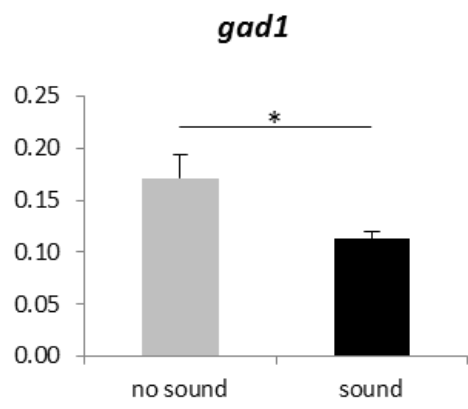

D vglut2

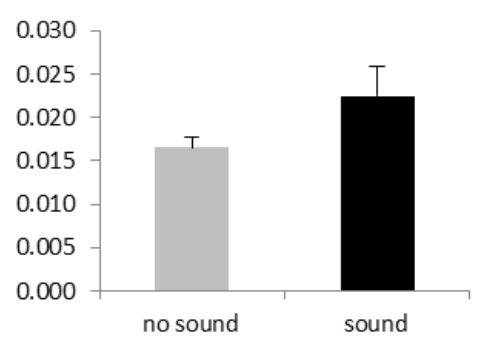

Figure 3.29: Effect of tone exposure on the cortical gene expression of genes involved in neuronal plasticity. Gene expression was measured by RT-qPCR. Relative expression of $\mathbf{A}$, cholinergic receptor muscarinic 2, chrm2; B, glutamic acid descarboxylase, gad1; C, GABA vesicular transporter, vgat; D, glutamate vesicular transporter 2, vglut2. Samples were collected at 7 days of sound exposure to $16 \mathrm{kHz}$. ${ }^{*} \mathrm{p}<0.05$.

In summary, the similarity in the expression of measured genes between the control and exposed groups suggests that the changes in gene expression are triggered by the Audiobox environment and not by relevant sound exposure itself.

It has been reported changes in the cortical expression of chrm2, gad1, vglut2 and vgat due to sound exposure in the AC (Browne et al. 2012; Lai et al. 1989) and enriched environment in the 
visual cortex (Mainardi et al. 2010). Therefore, I measured the expression of these genes in the AC. Compared to the control group $(n=6)$, the expression of the exposed group ( $n=6)$ of gad1 was reduced (Figure 3.29B; control, $0.17 \pm 0.02$ vs exposed $0.11 \pm 0.007, p=0.03$ ). No differences were found in the expression of chrm2 (control, $0.04 \pm 0.004$ vs exposed $0.04 \pm 0.005, p=0.65$ ), vgat (control, $0.18 \pm 0.03$ vs exposed $0.13 \pm 0.01, p=0.27$ ) and vglut2 (Figure 3.29A-D; control, $0.01 \pm$ 0.001 vs exposed $0.02 \pm 0.003, p=0.14)$. These results indicate that sound exposure in the Audiobox reduces specifically the expression of GAD in the auditory cortex.

\subsubsection{Expression of presynaptic markers in the IC}

To investigate whether the observed electrophysiological changes, induced by sound exposure, and the increase in the Vglut2/VGAT ratio at the level of gene expression were accompanied by molecular changes in protein expression of presynaptic molecules at particular locations of the IC, Olga Babaev (Neurobiology department, MPI-em, Göttingen) measured immunoreactivity to VGAT and Vglut2 proteins in the dorsal and ventral portion of the IC of control and exposed animals to 16 $\mathrm{kHz}$ during 6-12 days. We were particularly interested in the ratio between these two proteins as an expression of excitation/inhibition (E/I) balance, since it has been shown to be modified upon environmental manipulations and a common signature in cortical synaptic plasticity (Mainardi et al. 2014). The total number of Vglut2 and VGAT positive puncta was quantified for dorsal and ventral areas in each animal and the ratio between total number of VGAT and total number of Vglut2 was calculated (Figure 3.30A). We found that the amount of Vglut2 in the dorsal area was similar (Figure 3.29B; $119976 \pm 12940$ vs $130625 \pm 3907, p=0.68$, control and exposed to $16 \mathrm{kHz}$, respectively. $n=7$ for each group), while there was a significant reduction of VGAT for the exposed animals (Figure 3.30C; $92251 \pm 9406$ vs $69241 \pm 9864, p=0.03$, control and exposed, respectively). Therefore, we observed a significant increase in the Vglut2/VGAT ratio of exposed animals (Figure 3.30C; $1.5 \pm 0.15$ vs $1.9 \pm 0.27, p=0.03$, control and exposed, respectively). In the ventral areas, there was a decrease in Vglut2 for the exposed animals; while there was no change in the amount of VGAT (Figure 3.30F). The Vglu2/VGAT ratio was slightly higher for the exposed group ( $p>0.05$ ). This result indicates that relevant sound exposure increases the $\mathrm{E} / \mathrm{I}$ balance only in the dorsal part of the IC. 

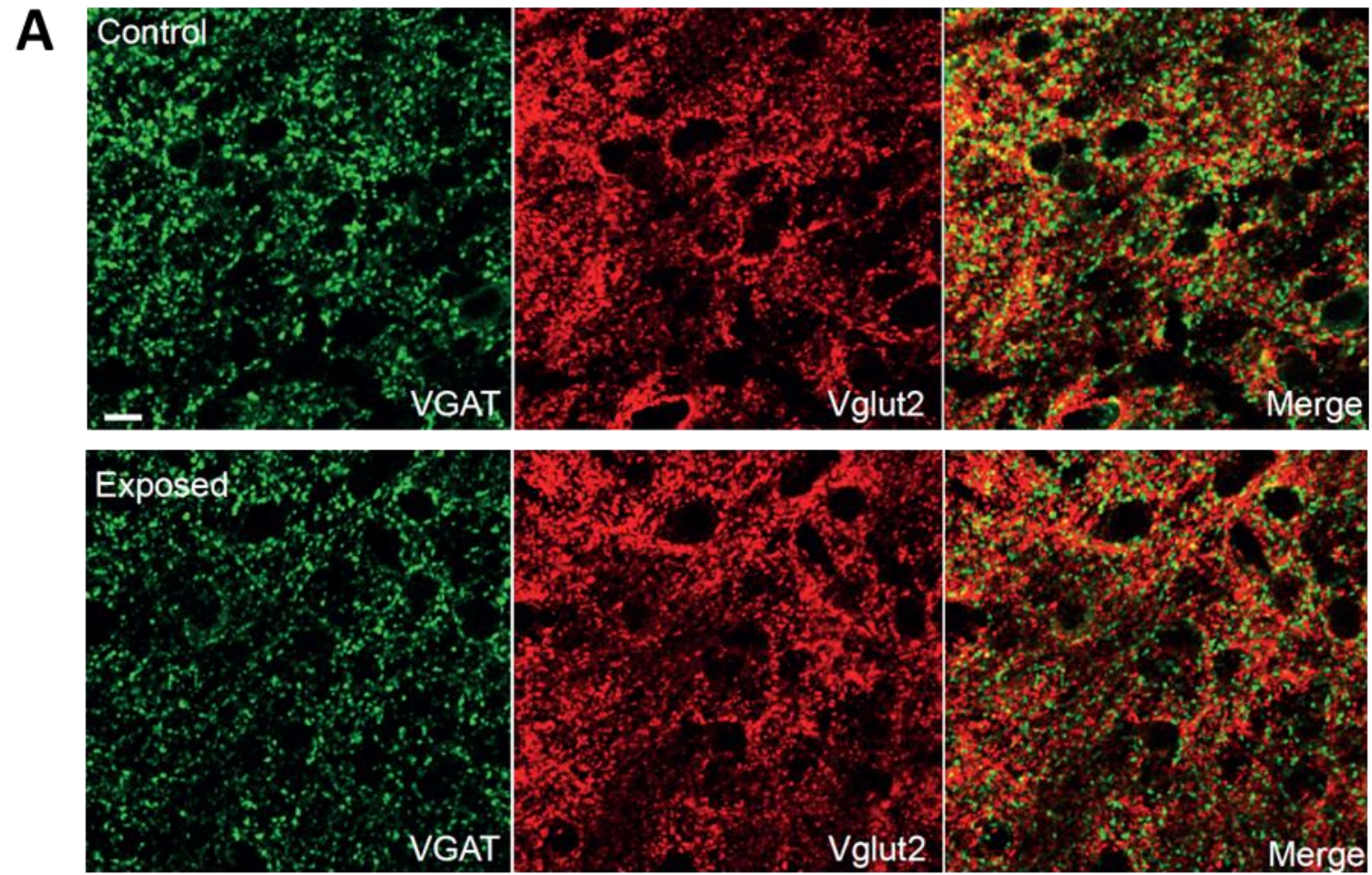

B

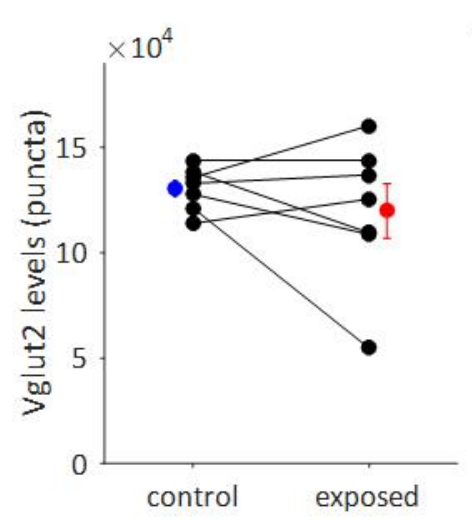

$\mathbf{E}$

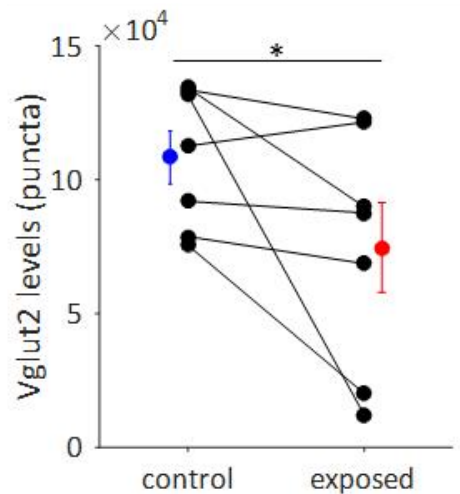

C

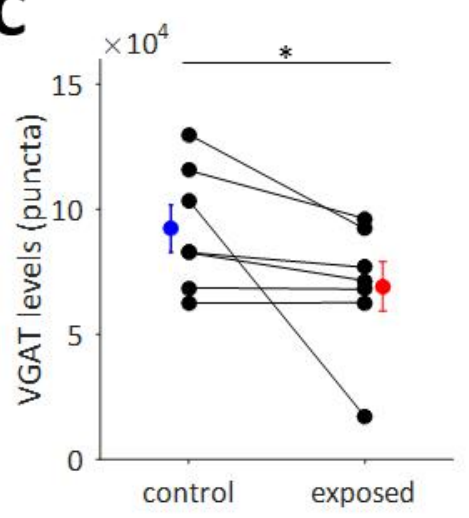

$\mathbf{F}$

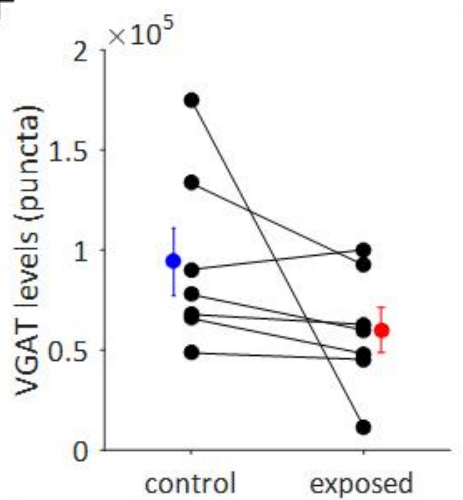

D

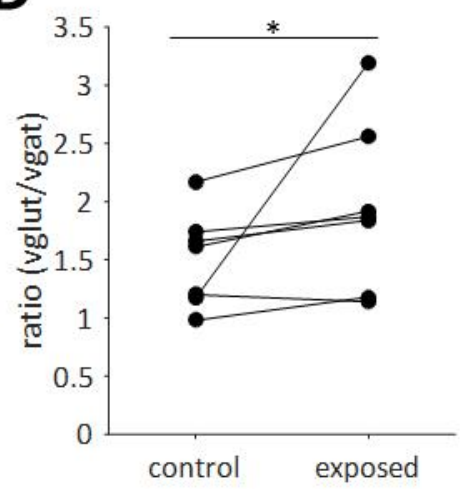

G

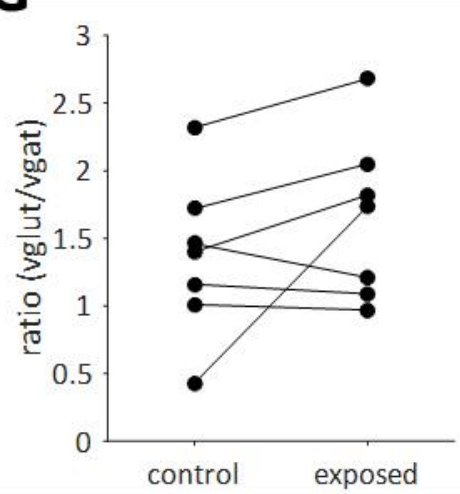

Figure 3.30: Effect of tone exposure on the collicular expression of presynaptic proteins. A, Representative photomicrographs of a section of the IC double labeled for VGAT and Vglut2 for control (upper panels) and exposed (lower panels) groups. Quantification of the positive puncta for Vglut2 in B, Dorsal and E, Ventral areas. Quantification of the positive puncta for VGAT in the C, Dorsal and F, Ventral areas. Ratios between Vglut2/VGAT in D, Dorsal and G, Ventral areas. Samples 
were collected between 6-12 days of sound exposure to $16 \mathrm{kHz} .{ }^{*} \mathrm{p}<0.05$. Data collected by Olga Babaev.

\subsection{Effect of sound exposure in the cochlear nucleus.}

We were interested in knowing whether the changes observed in the IC due to sound exposure were due to an overall plastic change along the entire auditory pathway, particularly at subcollicular nuclei. For that purpose, Dr. Zhizi Jing and Dr. Nikola Strenzke from the Auditory physiology lab in the Klinikum, Göttingen, performed single unit recordings in the cochlear nucleus of control $(n=6)$ and exposed animals $(8 \mathrm{kHz} ; \mathrm{n}=6)$ during $6-12$ days.

The tuning curves obtained from different types of units, the spike rates as well as the temporal response, were similar between groups (Figure 3.31). In summary, sound exposure did not induce changes in the sound processing for different types of units in the cochlear nucleus.
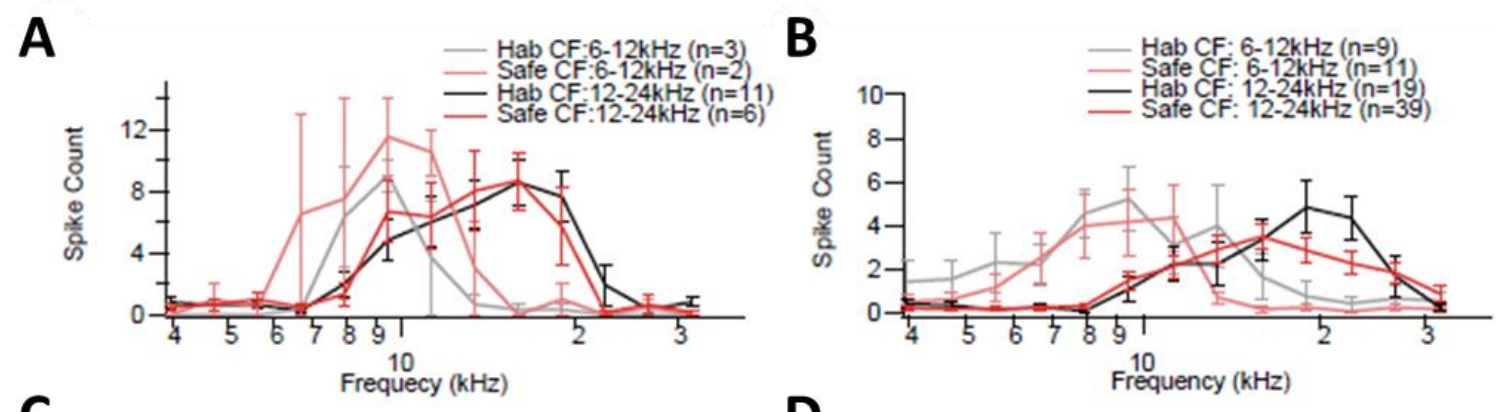

C

D
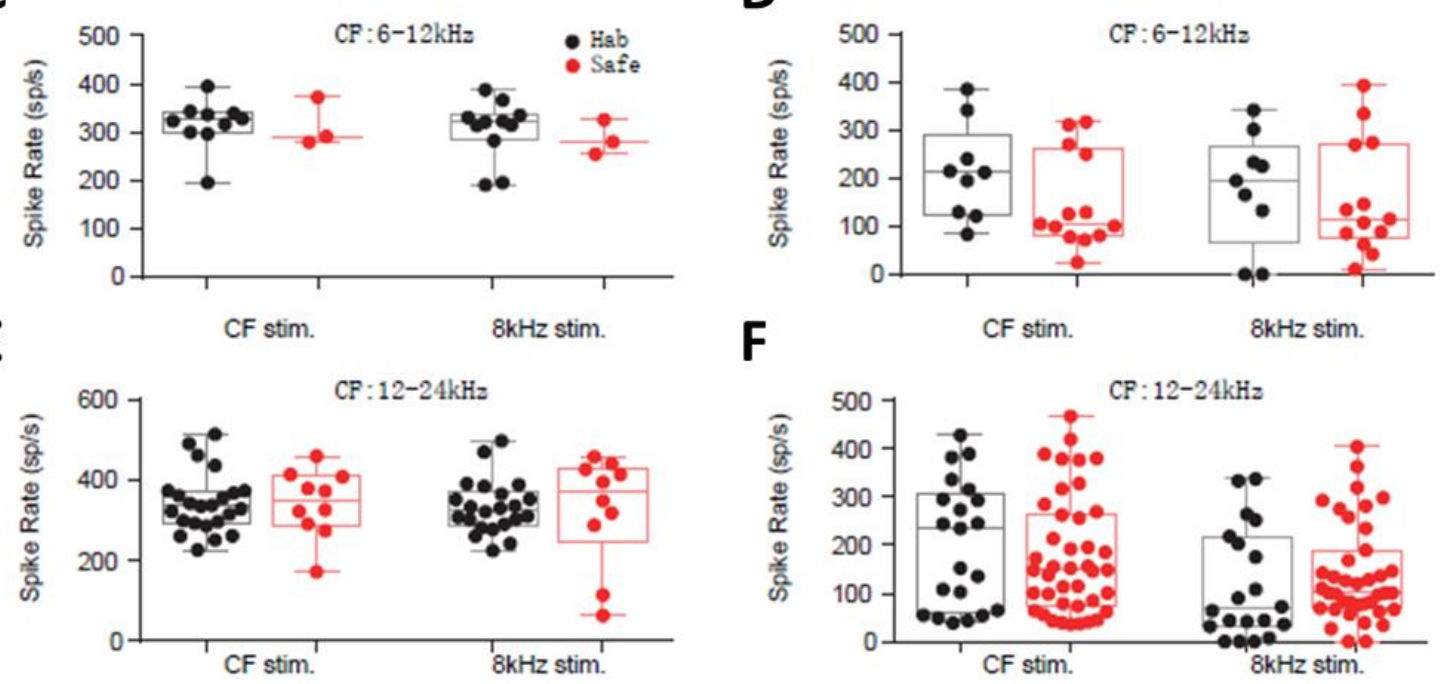

Figure 3.31: Effect of tone exposure on sound processing in the cochlear nucleus. A-B, Averaged tuning curves at $70 \mathrm{~dB}$. C-D, Evoked spike rate to the characteristic frequency (CF) and to $8 \mathrm{kHz}$ of units with a CF $6-12 \mathrm{kHz}$. E-F, Evoked spike rate to the $\mathrm{CF}$ and to $8 \mathrm{kHz}$ of units with a CF $12-24 \mathrm{kHz}$. Single units were classified as primarylike (PL), primarylike-with-notch (left panels) and other types (right panels). Recordings were made between 6-12 days of sound exposure to $8 \mathrm{kHz}$. Data collected by Dr. Zhizi Jing and Dr. Nicola Strenszke 


\subsection{Behavior}

\subsubsection{Pre-pulse inhibition of the acoustic startle response.}

At this level of the study, I was interested in evaluating whether the plastic changes observed in the IC due to sound exposure (frequency representation, amplitude of tuning curves, etc) were correlated with behavioral changes. Since changes in frequency discrimination have been correlated with changes in cortical sound representation (Weinberger 2004; Han et al. 2007; Froemke et al. 2013), I decided to test whether sound exposure in the Audiobox modified frequency discrimination. It was important to assess frequency discrimination acuity without training, to avoid possible induction of plasticity by the training itself and being able to correlate the behavioral readout with the changes already characterized in the IC. Pre-pulse inhibition (PPI) of the acoustic startle response (ASR) is a behavioral assay that has been used to successfully determine frequency discrimination acuity (FDA) in mice without the need of training (Clause et al. 2011; Aizenberg \& Geffen 2013a; Mwilambwe-Tshilobo et al. 2015; Aizenberg et al. 2015). The ASR is an innate reflex to loud sounds that can be inhibited if a short pre-pulse is delivered before the loud sound. If PPI is assessed with a constant background tone the inhibition of the ASR will be proportional to the difference between the background tone and the pre-pulse tone (see Figure 2.3). I tested animals that were exposed to $16 \mathrm{kHz}$ with every visit to the corner (exposed group); animals exposed to 16 $\mathrm{kHz}$ in the homecage (random group) and control animals that lived in the Audiobox the same amount of time as the other groups but without sounds (control group). The experiments were carried between 7 to 11 days of sound exposure and the animals were tested only once. On each day a few animals of each group were tested. In this experiment the mean daily length of the visits to the corner was similar between the relevant-exposed group and the mouse that activated the sound in the homecage for the random group (1378 \pm 184 and $1335 \pm 40 \mathrm{~s}$, respectively, $p=0.806$ ), indicating that animals from both groups heard $16 \mathrm{kHz}$ during similar amount of time.

Moreover, when I analyzed the group data of all the PPI values for each $\Delta f$, the exposed group was significantly different from the control and random exposed animals (Figure 3.32A; $F_{2,8}=13.59$, $p<0.0001 ; p<0.001$ vs control and random exposed groups). For the analysis I used the same methodology previously described by Geffen \& Aizenberg, 2013. By fitting a generalized logistic function of the PPI values for each animal, I calculated the individual discrimination thresholds (Th) defined as the shift in frequency that caused $50 \%$ of inhibition of the maximum response (see Methods 2.3.4). Animals with a fit coefficient below 0.7 were excluded from the analysis ( 2 control 
animals, 3 exposed animals and 1 random animal, out of 10 for each group). The individual Th and the mean value for each group are plotted in the Figure 3.32B. The thresholds of the

A

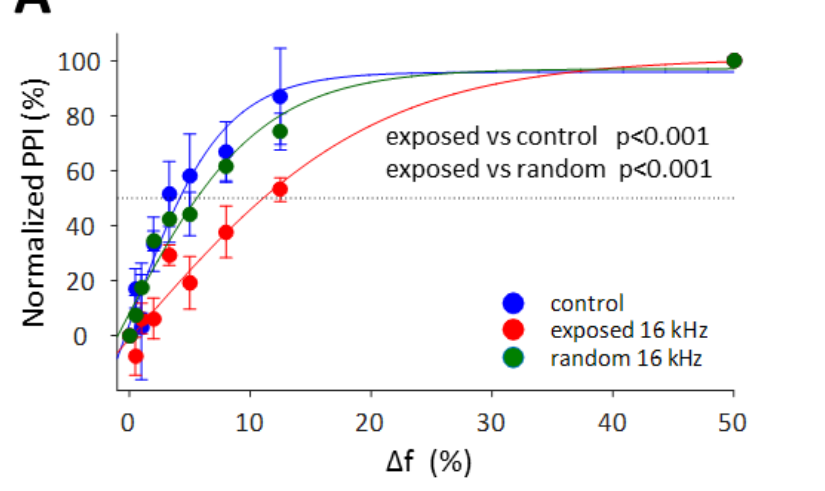

B

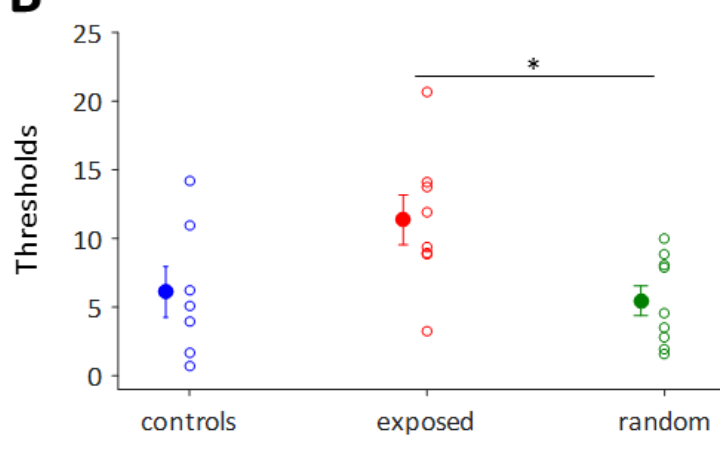

Figure 3.32: Effect of tone exposure on frequency discrimination acuity. A, PPI as function of frequency change between the pre-pulse and the background tone for control (blue), exposed (red) and random (green) groups. The exposure frequency was $16 \mathrm{kHz}$. Color lines indicate the fitted curve for each group. B, Average discrimination thresholds for control, exposed and random groups. ${ }^{*} \mathrm{p}<0.05$.

control and random group were very similar ( $6.1 \pm 1.8$ vs $5.4 \pm 1 \% \Delta f)$. The average Th between groups was different (Figure 3.32B; $F_{2}=4.32, p=0.02$ ). The average Th of the relevant exposed animals was higher than control animals but did not reach statistical significance, due to very low value in the exposed group ( $11.35 \pm 1.8$ vs $6.1 \pm 1.8 \% \Delta f, n=8$ and 7 , respectively, $p=0.097$ ). However, the average Th of the exposed group was significantly higher than random exposed animals (11.35 \pm 1.8 vs $5.4 \pm 1 \% \Delta f, n=8$ and 9 , respectively, $p<0.05)$.

In summary, these results indicate that relevant but not random exposure inside the corner of the Audiobox decreases FDA around the exposed frequency.

\subsubsection{Latent inhibition}

Once established that sound exposure decreases FDA around the exposed frequency, but only when it occurs inside the corner, I was interested to test whether sound exposure will also affect subsequent learning. Particularly, I was interested to test how long it will take for the exposed and random animals to learn the change in the behavioral value of the exposed sound (from safe or neutral to punishment signal). For this purpose I used the latent inhibition paradigm. Latent inhibition is defined as the delay in learning due to pre-conditioned exposure of what later will be the conditioned stimulus. The latent inhibition paradigm in the Audiobox consisted of four different phases: habituation ( 3 days; no sound), safe ( 7 days; $8 \mathrm{kHz}$ tone pips were paired with visits to the 
corner), pre-conditioned ( 5 days, explained in detail below) and conditioned ( 5 days; animals had to learn to

A

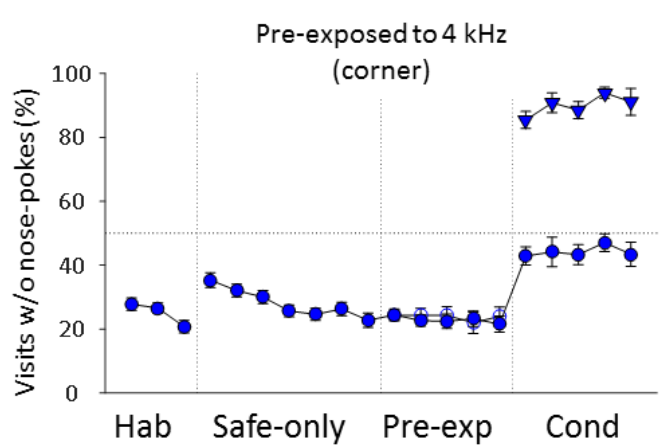

C

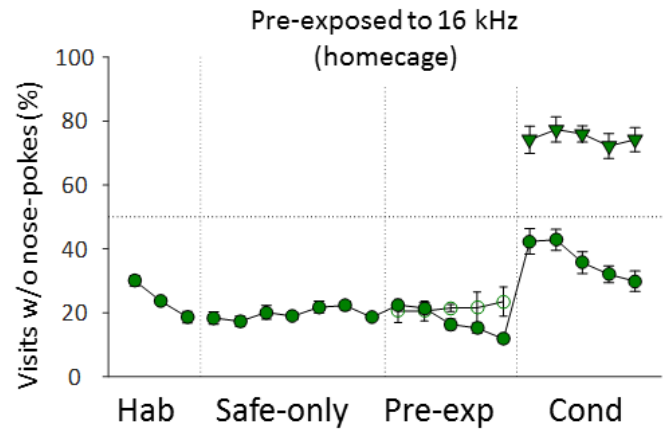

B

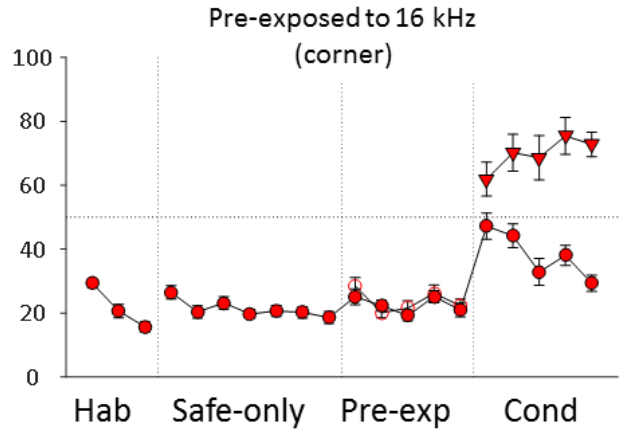

D

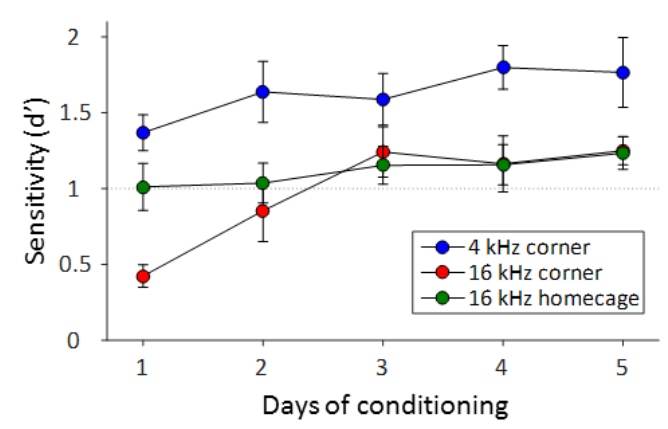

Figure 3.33: Effect of tone exposure during the pre-conditioned phase on the expression of latent inhibition. Daily performance (expressed in \% of visits without nose-pokes) of mice during different training phases of the latent inhibition paradigm. Filled circles represent habituation or safe visits paired with $8 \mathrm{kHz}$, open circles are visits paired with the pre-exposed tone. Animals were preexposed $17 \%$ of the visits to different tones during the pre-conditioning phase: $\mathbf{A}, 4 \mathrm{kHz}$ in the corner (control group); B, $16 \mathrm{kHz}$ in the corner (LI group); or C, $16 \mathrm{kHz}$ in the homecage (random exposed group). Filled triangles represent the conditioned visits to $16 \mathrm{kHz}$ where animals in case of nose-poking received an air-puff. D, Discriminability index ( $d$ ') as a function of the conditioning day for the three groups of animals.

nose-poke in the corner only when $8 \mathrm{kHz}$ was presented and to avoid nose-poking when $16 \mathrm{kHz}$ was presented) (Figure 2.2; see Methods 2.3.1). Only the pre-conditioned phase was different between groups. During this phase two different tones were presented. For the control group, in $71 \%$ of the visits to the corner $8 \mathrm{kHz}$ was played and in $29 \%$ of the visits, $4 \mathrm{kHz}$ was played. For the latent inhibition group (LI), 8 and $16 \mathrm{kHz}$ were played (71 and 29\% respectively). For the random group, 8 $\mathrm{kHz}$ was played in $100 \%$ of their visits to the corner and $16 \mathrm{kHz}$ was played in the homecage (see Methods 2.3.1). During the safe and pre-conditioning phases the tones played were safe sounds, meaning that the animals could nose-poke without punishment. On average, animals did not nosepoke in $20-30 \%$ of their visits to the corner (Figure 3.33A-C). During the safe phase, control animals 
showed lower proportion of visits with nose-pokes during the first 4 days $(n=15)$. However, by the end of the safe phase, the performance was similar between groups. Addition of a second tone during the pre-conditioned phase did not alter the performance in the control and LI groups ( $n=19)$. The random exposed group $(n=20)$ showed a lower number of visits with nose-pokes in the last 2 days previous to the beginning of the conditioned phase. During the pre-conditioned phase, the average daily length of time spent in the corner was similar for the mice in the LI group and the mice that activated the sound for the random exposed group in the two replications that were made (432 \pm 41 and $338 \pm 35 \mathrm{~s}$, respectively), indicating that the animals from these two groups heard $16 \mathrm{kHz}$ in the pre-conditioned phase during similar amount of time. During the conditioned phase, the control mice (exposed to $4 \mathrm{kHz}$ during pre-conditioning phase) learned to discriminate the conditioned tone $(16 \mathrm{kHz})$ within the first day, with an average of $85 \pm 3 \%$ of the conditioned visits without nose-pokes (Figure 3.33A). As expected, it took longer for the LI group (exposed to 16 $\mathrm{kHz}$ in the corner during pre-conditioning phase) to learn to avoid nose-poking during conditioned visits (Figure 3.33B). On average animals of the LI group avoided nose-poking in $62 \pm 5 \%$ of the conditioned visits, thus expressing latent inhibition. The random group (exposed to $16 \mathrm{kHz}$ in the homecage during pre-conditioning phase) showed an intermediate level between the control and Ll group, with an average of $74 \pm 4 \%$ of the conditioned visits without nose-pokes (Figure $3.33 \mathrm{C}$ ). By the second day of conditioning, the proportion of conditioned visits without nose-pokes of the control animals slightly increased to $90 \pm 3 \%$, while the $\mathrm{LI}$ and random groups was $70 \pm 6$ and $77 \pm 4 \%$ respectively. It was not until the fourth day of conditioning that the proportion between $\mathrm{LI}$ and random groups became similar ( $75 \pm 5$ and $72 \pm 4 \%$, respectively).

For all groups there was also an increase in the proportion of safe visits without nose-pokes $(47 \pm 4$, $42 \pm 4$ and $42 \pm 3 \%$, for control, LI and random exposed, groups, respectively), that gradually decrease for the LI and random groups down to $30 \pm 3 \%$ for both groups. The safe visits without nose-pokes for control animals remained around $42 \pm 4 \%$ along the conditioning days.

We calculated the discriminability index ( $d$ ', see Methods 2.3.2) for each group across conditioning days (Figure 3.33D). A d' of 1 or higher indicates that the animals could discriminate between safe and conditioned visits. Control animals showed an index above 1 during the 5 days of conditioning. In contrast, LI animals had average d' values below 1 during the first two days ( 0.42 and 0.85 , respectively) indicating that they could not discriminate until the third day. Random exposed animals had average $d^{\prime}$ values of 1 or above from the first day of conditioning indicating that indeed they could discriminate since day 1 . The average d' of the LI group were significantly different from the control and random exposed group on day $1(0.42 \pm 0.07$ vs $1.01 \pm 0.15$ for $\mathrm{LI}$ and random, 
respectively, $\mathrm{p}<0.05)$ and from the control group on day $2(0.85 \pm 0.2$ vs $1.67 \pm 0.2$, for $\mathrm{LI}$ and control, respectively, $\mathrm{p}<0.05)$.

In summary, it took longer for animals to learn the conditioned value of a sound when it was preexposed inside the corner, strongly suggesting that the animals indeed assign a behavioral relevance to it, contrary to the case in which the sound was pre-exposed in the homecage.

\subsection{Electrophysiology after conditioning}

Since it has been shown that there are short term plastic changes in the IC after fear conditioning in bats (Ji et al. 2005; Suga et al. 2002), I was interested in knowing whether our training paradigm (two-tone discrimination task) could also modify frequency representation in the IC and the effect of latent inhibition.

I performed recordings with multi-electrode arrays (4 shanks $x 4$ electrodes) in the IC of animals from one replication of the latent inhibition experiments showed in the section 3.4.2. These animals, after 3 days of habituation without sounds, went through a safe phase (7 days) where 8 $\mathrm{kHz}$ was played in visits to the corner; a pre-conditioned phase (5 days) where they were preexposed to 8 and $16 \mathrm{kHz}$, and a conditioning phase (5 days). In the conditioning phase animals learnt to nose-poke in the corner only when $8 \mathrm{kHz}$ (safe tone) was presented and to avoid nose-poking when $16 \mathrm{kHz}$ was presented (conditioned tone). One group of animals were from the $\mathrm{LI}$ group (Figure 3.33B, pre-conditioned exposure to 8 and $16 \mathrm{kHz}, \mathrm{n}=6,259$ recorded sites) and the other group of animals were from the random group (Figure 3.33C, pre-conditioned exposure to $8 \mathrm{kHz}$ in the corner and $16 \mathrm{kHz}$ in the homecage, $\mathrm{n}=7,311$ recorded sites). The recordings started after five days of conditioning to $16 \mathrm{kHz}$, at this time, both groups showed similar discriminability indexes (d') (Figure 3.33D; $1.25 \pm 0.09$ vs $1.23 \pm 0.1$, for $\mathrm{LI}$ and random groups, respectively).

\subsubsection{Sound processing in the IC after conditioning}

The Figure 3.34 shows the average BF per depth for each group (LI group, magenta traces; random group green traces) along the rostro-caudal axis (A to D). The average BF of a non-conditioned control group is shown in blue as a reference (the values for this group are the same as in Figure 3.12). The average BF of the LI group are higher than the random group in ventral depths (400-750 $\mu \mathrm{m}$ ) of the rostral and medial portion of the IC (Figure 3.34 A-B; Rostral: $F_{1,13}=30.05 p<0.001$, groupdepth interaction $F_{1,13}=1.51 ; p=0.11$; Central 1: Figure 3.34B; $F_{1,13}=6.21 p=0.01$, group-depth interaction $\mathrm{F}_{1,13}=1.05 ; \mathrm{p}=0.4$ ). There was no significant difference in the central 2 (Figure 3.34C; $F_{1,13}=3.83 p=0.053$, group-depth interaction $F_{1,13}=0.7259 ; p=0.73$ ) and caudal part (Figure 3.34D; $F_{1,13}=1.64 p=0.2$, group-depth interaction $F_{1,13}=0.9 ; p=0.55$ ). 
In the $A C$ and IC a specific increase in the responses to the conditioned tone of fear conditioned animals has been shown (Bakin \& Weinberger 1990; Gao \& Suga 2000). Therefore, I analyzed the

A

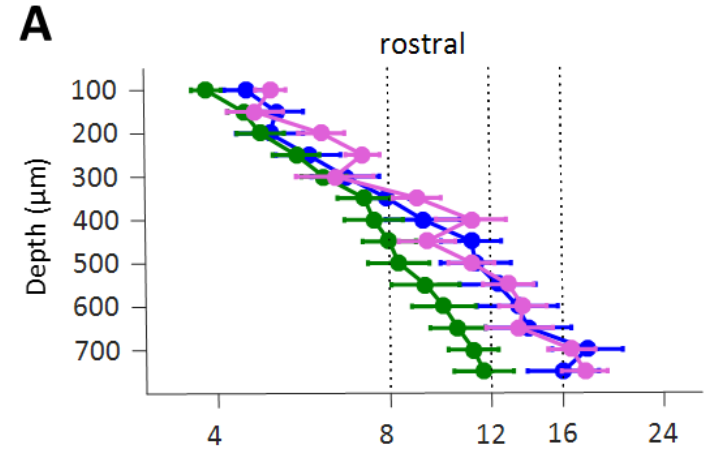

C

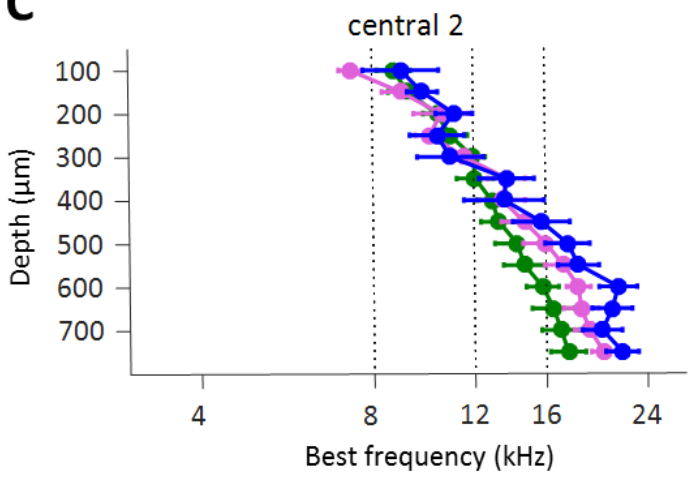

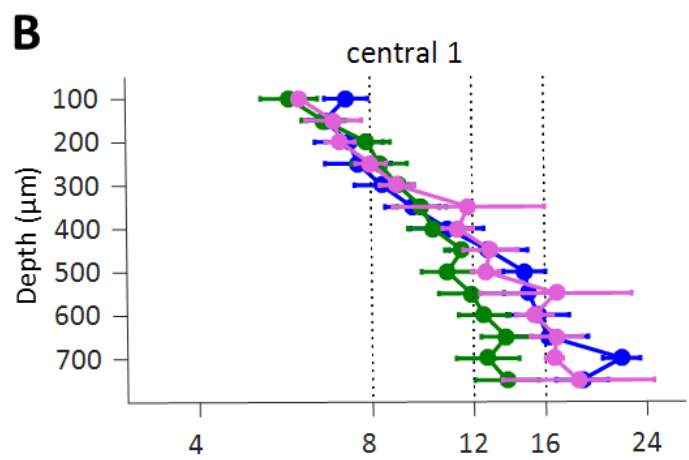

D

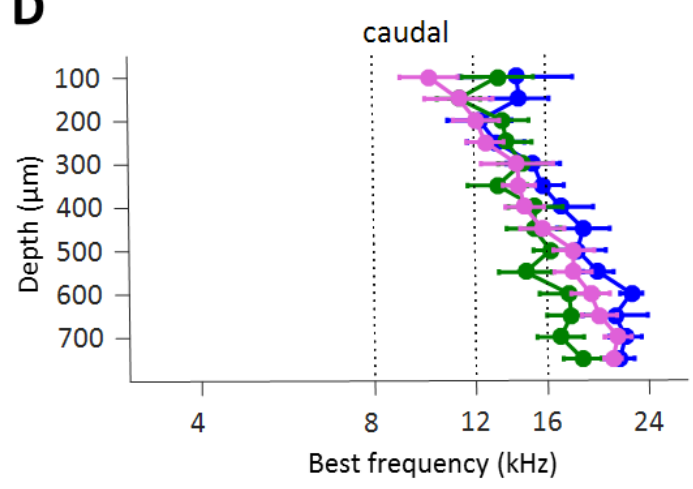

control untrained - pre-exposed in corner $16 \mathrm{kHz}$

- pre-exposed in homecage $16 \mathrm{kHz}$

Figure 3.34: Effect of conditioning on collicular tonotopy. A-D, Average best frequencies along the dorso-ventral axis from different rostro-caudal locations of the IC of animals conditioned to $16 \mathrm{kHz}$. Before conditioning, the animals were pre-exposed to $16 \mathrm{kHz}$ in the corner (magenta) or in the homecage (green). Recordings were obtained during 6 to 10 days after the animals from both groups learned the task and the discrimination indexes were similar. Average best frequencies of the control non-conditioned group were plotted as reference (blue).

responses to the conditioned tone $(16 \mathrm{kHz})$ in the IC. The Figure 3.35 shows the average response to $16 \mathrm{kHz}$ per depth for each group (LI group, magenta traces; random group green traces) along the rostro-caudal axis ( $A$ to $D$ ). The average BF of a non-conditioned control group is shown in blue as a reference. The average responses between groups were similar in the rostral (Figure 3.35A; $F_{1,13}=0.58, p=0.44$, group-depth interaction $F_{1,13}=0.66, p=0.8$ ), central 1 (Figure 3.35B; $F_{1,13}=0.05$ $p=0.8$, group-depth interaction $F_{1,13}=1.25, p=0.25$ ) and central 2 (Figure $3.35 C ; F_{1,13}=0.8 p=0.3$, group-depth interaction $F_{1,13}=0.1, p=1$ ) along the dorso-ventral axis. In contrast the average responses were higher in the random group compared to the LI group in the caudal part (Figure 3.35D; $F_{1,13}=15.76 p<0.001$, group-depth interaction $F_{1,13}=1.54, p=0.1$ ). 
Interestingly, the average responses to the safe tone $(8 \mathrm{kHz})$, shown in the Figure 3.36 , for random animals were higher in the rostral areas compared to the $\mathrm{LI}$ group (Figure 3.36A; $F_{1,13}=19.84$, $p<0.0001$, group-depth interaction $F_{1,13}=0.3, p=0.9$ ), central 1 (Figure 3.36B; $F_{1,13}=4.6, p=0.03$, group-depth interaction $\mathrm{F}_{1,13}=0.5, \mathrm{p}=0.9$ ), while the most caudal parts were similar between groups (Figure

A

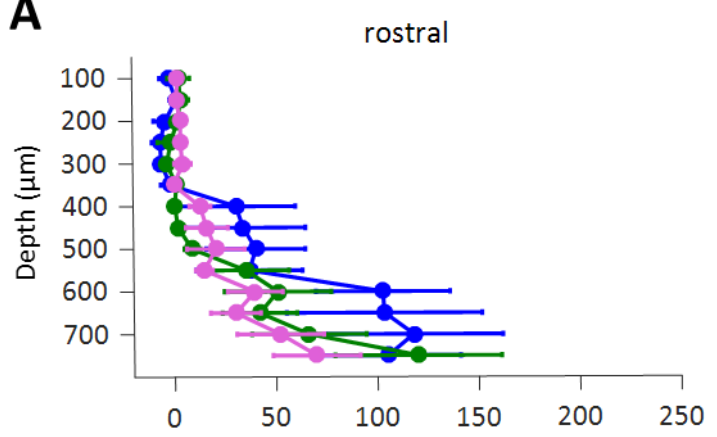

C

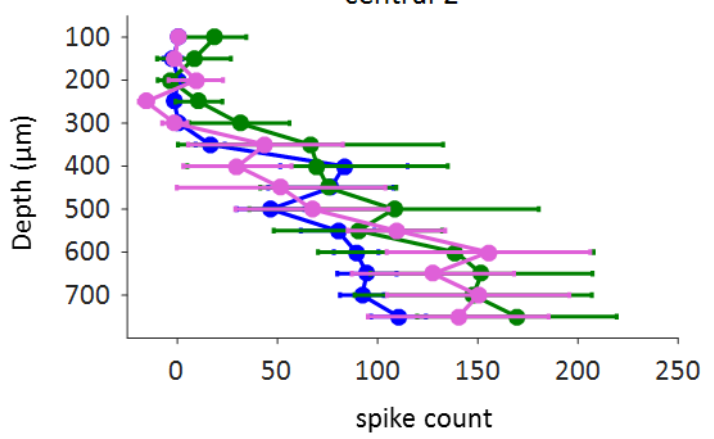

B

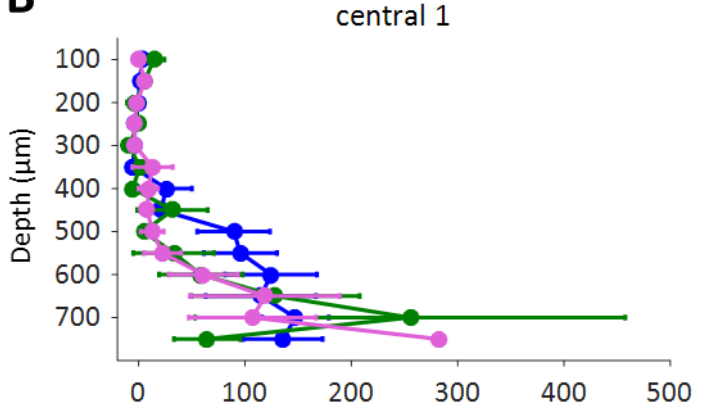

D

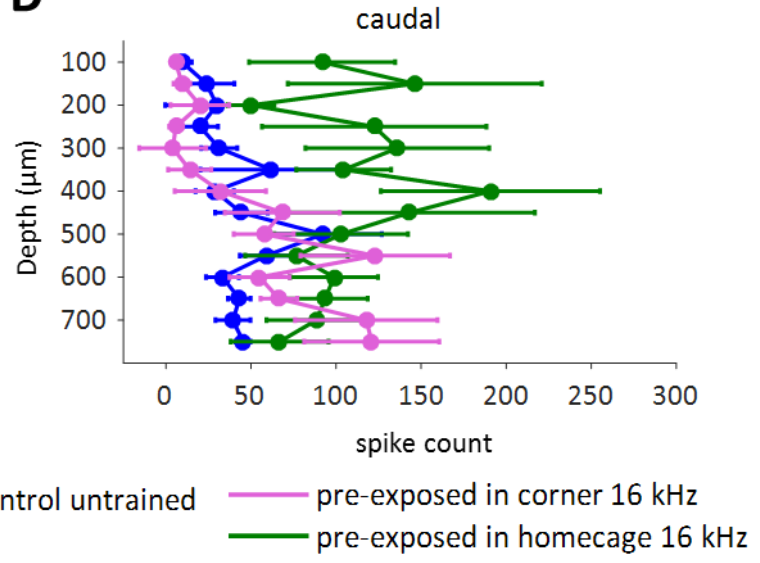

Figure 3.35: Effect of conditioning on the responses to the conditioned tone (16 kHz) in the IC. AD, Average responses to the conditioned tone $(16 \mathrm{kHz})$ along the dorso-ventral axis from different rostro-caudal locations of the IC of animals conditioned to $16 \mathrm{kHz}$. Before conditioning, the animals were pre-exposed to $16 \mathrm{kHz}$ in the corner (magenta) or in the homecage (green). Recordings were obtained during 6 to 10 days after the animals from both groups learned the task and the discrimination indexes were similar. Average values of the control non-conditioned group (blue) were plotted as reference.

3.36C,D; central 2: $F_{1,13}=0.5, p=0.4$, group-depth interaction $F_{1,13}=0.1, p=0.9$; caudal: $F_{1,13}=0.7 p=0.4$, group-depth interaction $\mathrm{F}_{1,13}=0.4, \mathrm{p}=0.9$ ).

To characterize the temporal firing pattern of the $\mathrm{LI}$ and random groups to the conditioned and safe tones, I selected the depth with the best response to 8 or $16 \mathrm{kHz}$ in each of the four rostro-caudal positions, and compared the average PSTH ( 5 ms bins) between groups. The temporal response was divided in onset (0-30 ms after sound onset) and delayed (31-80 ms after sound onset) responses, as in the previous analysis of the section (3.1.2). The onset component of the average responses to 
the conditioned tone $(16 \mathrm{kHz})$ was different between random and $\mathrm{LI}$ groups in the central portion of the IC (Figure 3.37B; $\mathrm{p}=0.04$ and 0.03 for central 1 and central 2, respectively). In the caudal portion, the delayed component was longer in the random group compared to the $\mathrm{LI}$ group $(p=0.0002)$. Compared to the control non-conditioned group, the average responses in the onset component of the LI group
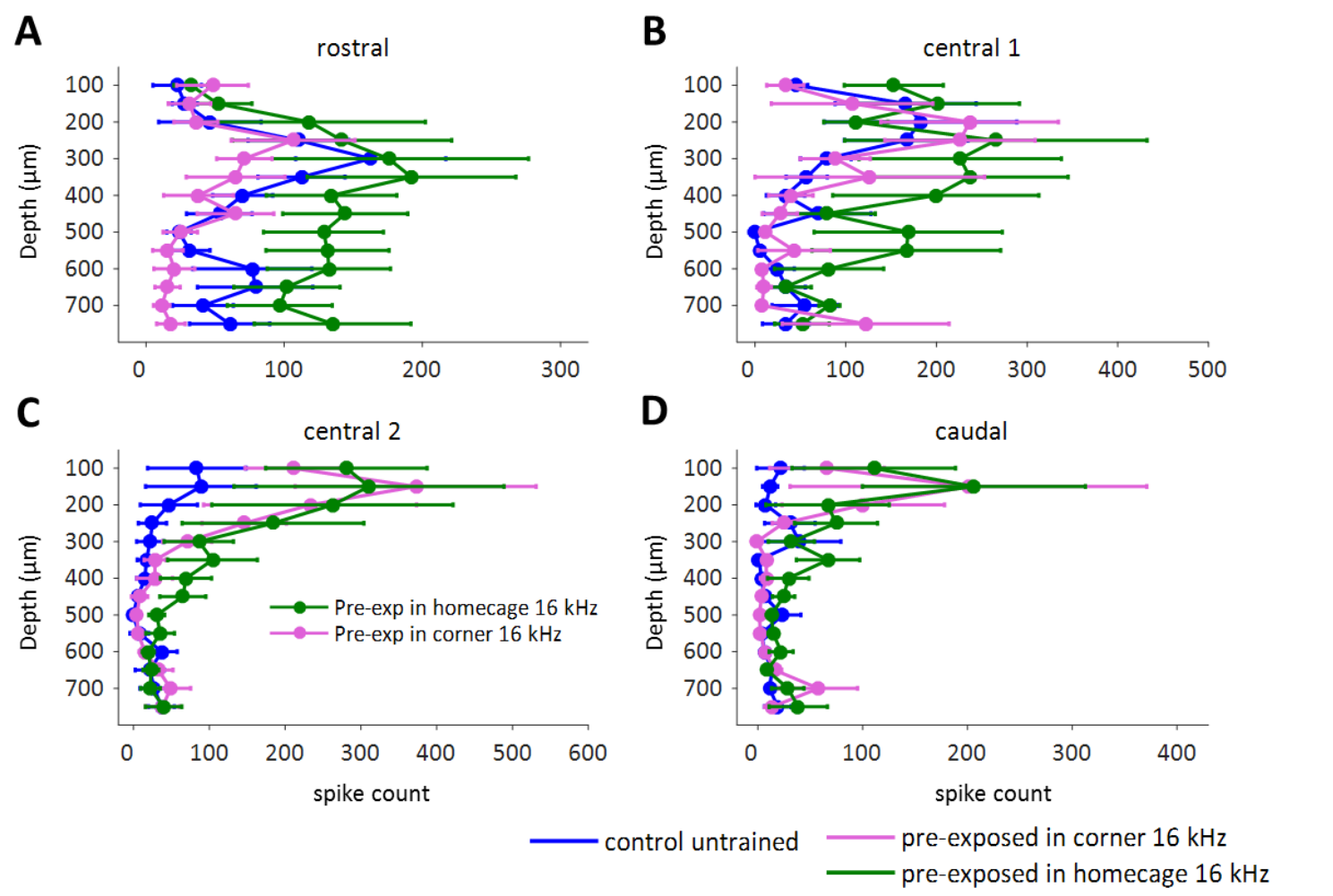

Figure 3.36: Effect of conditioning on the responses to the safe tone $(8 \mathrm{kHz})$ in the IC. A-D, Average responses to the safe tone $(8 \mathrm{kHz})$ along the dorso-ventral axis from different rostro-caudal locations of the IC of animals conditioned to $16 \mathrm{kHz}$. Before conditioning, the animals were preexposed to $16 \mathrm{kHz}$ in the corner (magenta) or in the homecage (green). Recordings were obtained during 6 to 10 days after the animals from both groups learned the task and the discrimination indexes were similar. Average values of the control non-conditioned group (blue) were plotted as reference.

and the average delayed responses of the random group were different in the caudal part of the IC (Figure 3.37B; $\mathrm{p}=0.0045$ and $\mathrm{p}=0.0014$, for $\mathrm{LI}$ and random group, respectively). The onset component of the average responses to the safe tone $(8 \mathrm{kHz})$ was slightly higher in the rostral and caudal portions of the IC of the random group compared to the LI group (Figure 3.37A; $p=0.07$ ). Compared to the control non-conditioned group, the onset and delayed responses of both trained groups were larger in the medial and caudal part of the IC (Figure 3.37A; central 2 and caudal, $\mathrm{p}<0.05)$. 
In summary, these results indicate that previous sound exposure has a long lasting effect in frequency representations in the IC. Additionally, the random group showed larger responses to the safe tone in the rostral part and larger responses for the conditioned tone in the caudal part. In the caudal part the firing pattern to the conditioned tone was also different between the trained groups, with larger peak response for the LI group and a larger delayed response for the random group.

A Response to the safe tone $(8 \mathrm{kHz})$
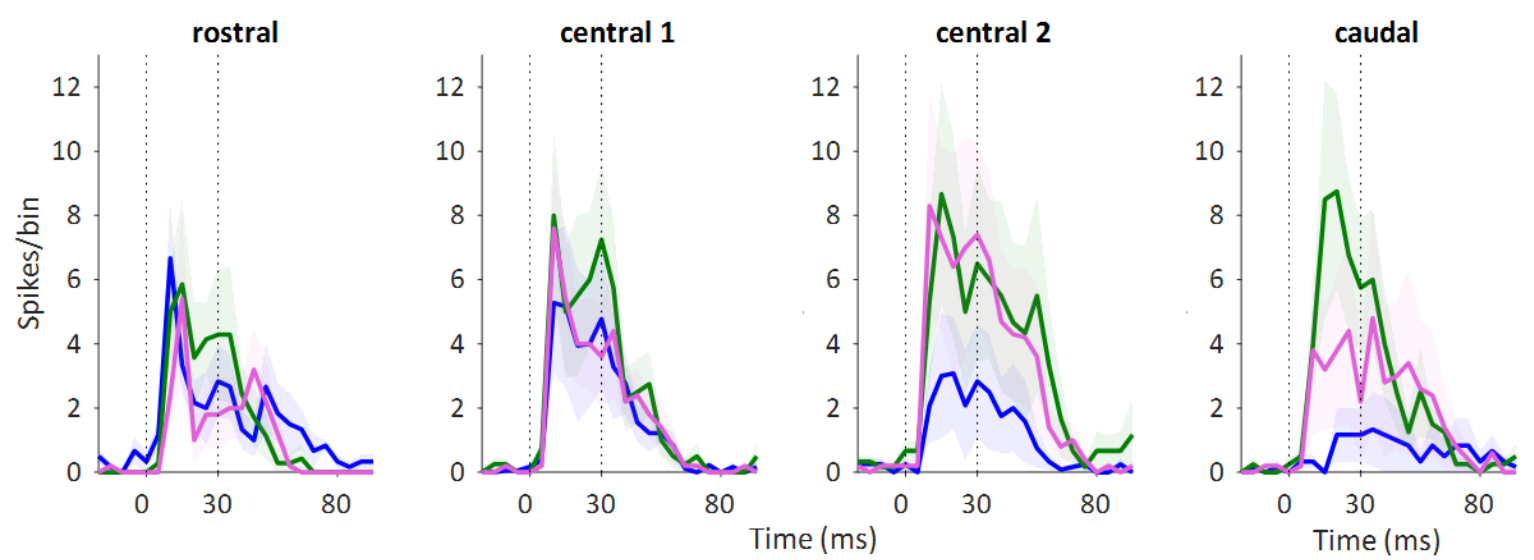

B

Response to the conditioned tone $(16 \mathrm{kHz})$
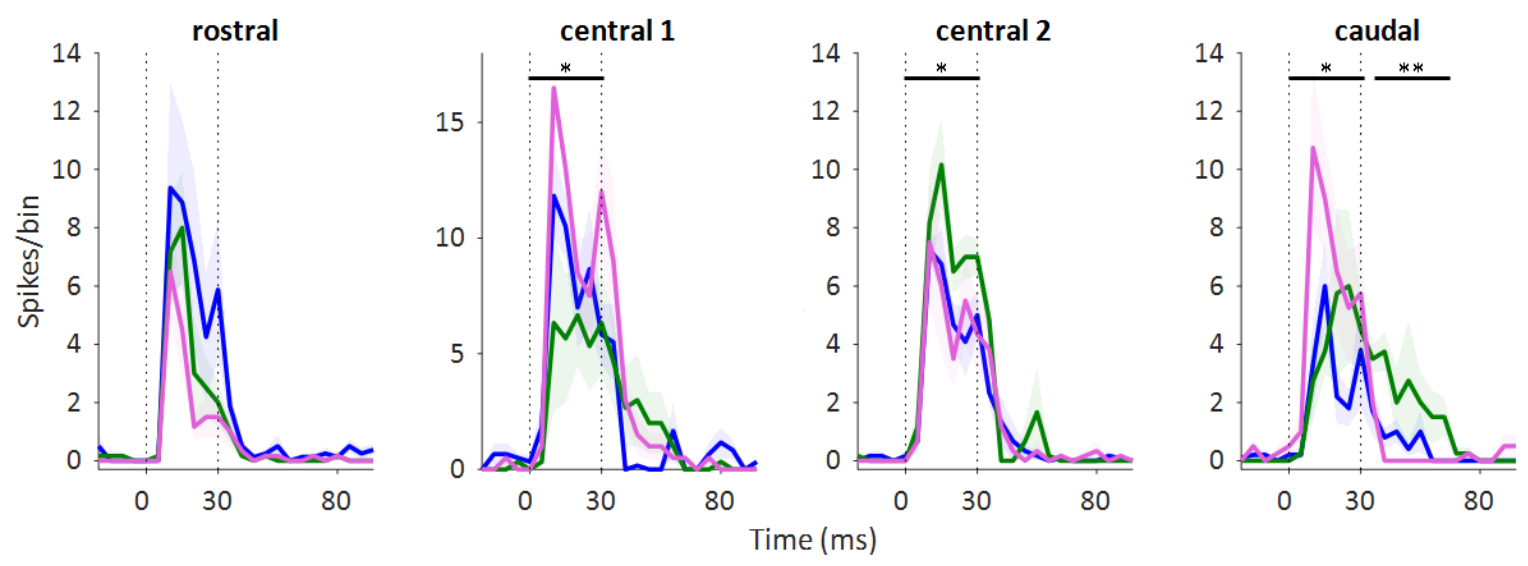

- control untrained

pre-exposed in corner $16 \mathrm{kHz}$ pre-exposed in homecage $16 \mathrm{kHz}$

Figure 3.37: Effect of conditioning on the firing pattern along the rostro-caudal axis of the IC. Average PSTH ( $5 \mathrm{~ms}$ bins) of the depth with the best responses at different rostro-caudal locations, for pre-exposed animals in the corner (magenta) and pre-exposed animals in the homecage (green) to $\mathbf{A}$, the safe tone $(8 \mathrm{kHz})$; and $\mathbf{B}$, the conditioned tone $(16 \mathrm{kHz})$, at $70 \mathrm{~dB}$ SPL. Recordings were obtained during 6 to 10 days after the animals from both groups learned the task and the discrimination indexes were similar. Vertical dashed lines indicate tone onset and delayed. The average PSTH of control non-conditioned animals (blue) were plotted as reference. ${ }^{*} p<0.05$ $* * p<0.001 \mathrm{LI}$ vs random. 


\subsection{Regulation of auditory cortical plasticity by blocking c-Fos expression}

Additional to my main project, I collaborated in a study that explored the role of c-Fos and its direct contribution to regulate discrimination-driven auditory cortical plasticity. Specifically, I contributed with electrophysiological data, by performing acute recordings in the auditory cortex of naïve and trained mice in the Audiobox treated with a short hairpin RNA that blocked c-fos expression. Here, I presented a brief version of the study, currently under revision.

Blocking c-Fos expression reveals the role of auditory cortex plasticity in sound frequency discrimination learning

Livia de $\mathrm{Hoz}^{1,2}$, Dorota Gierej ${ }^{1,3}$, Victoria Lioudyno ${ }^{3}$, Jacek Jaworski ${ }^{4}$, Magda Blazejczyk ${ }^{4}$, Hugo Cruces-Solís ${ }^{2,5}$, Anna Suska ${ }^{3}$, Tomasz Lebitko ${ }^{3}$, Tomasz Nikolaev ${ }^{3}$, Ewelina Knapska ${ }^{3, @}$, Israel Nelken $^{6, @}$, Leszek Kaczmarek ${ }^{3, @ ~}$

\section{Abstract}

Operant learning is associated with plasticity in early sensory cortices as well as with modulation of gene expression, but the connection between the behavioral, molecular and electrophysiological changes is only partially understood. Here we causally connect the three levels by showing that locally blocking c-Fos expression in mouse auditory cortex resulted in decreased cortical experience-dependent plasticity and a parallel and specific behavioral deficit in a sound discrimination task. c-Fos expression blockade had not effect on baseline excitability or basic auditory processing. Thus, experience-driven c-Fos expression is necessary for experiencedependent plasticity in the auditory cortex and c-Fos-dependent cortical plasticity is necessary for frequency discrimination assessment in an operant behavioral task.

\section{Results}


Mice lived in the Audiobox and had to learn to avoid nose-poking when the conditioned tones where present. Performance became better over time (Figure 3.38b). As expected, sound frequency discrimination learning resulted in a clear increase in c-Fos expression in the auditory cortex (Figure 3.38c). Experience-dependent plasticity was assessed with in vivo acute electrophysiology in animals that had either been trained in the Audiobox or had spent the same amount of time in their homecage. We found that experience-dependent plasticity was impaired in LV_sh_c-fos mice. Training led to an increase in sound-evoked neuronal activity, as well as sharpening of tuning curves in auditory cortex of LV_sh_luc but not LV_sh_c-fos mice (Figure 3.39a). Peri-stimulus time histograms for responses to 'safe' and 'conditioned' tones in trained animals show that onset responses were weaker in the LV_sh_c-fos mice (Figure 3.39b). The differences in the onset tuning were partly due to increased number of failures (trials which elicited no spikes) in LV_sh_c-fos mice (Figure 3.39c). Tuning of the late response (50-200 ms) was not different between the trained groups (Figure 3.39d). Stimulus specific adaptation (SSA), a reduction in neural activity caused by repeated presentation of a stimulus that does not generalize to other stimuli(Ulanovsky et al. 2003; Taaseh et al. 2011), was comparable between groups. SSA indices quantify the difference in the responses to a given frequency when deviant (rare, 5\% of the trials) and when standard ( $95 \%$ of trial; see methods) and confirmed that deviance detection was intact in cortical neurons of LV_sh_c-fos mice (Figure 3.39e-f). Local field potentials, which reflect the cortical input, were also comparable between the two groups (Figure 3.39g).

Impaired experience-dependent plasticity in LV_sh_c-fos mice was accompanied by learning deficits in tone discrimination in the Audiobox. Another group of mice was injected with either LV_sh_c-fos or LV_sh_luc. The mice were then trained in the Audiobox (Figure 3.40a) such that after a phase of only safe visits, the conditioned visits were introduced gradually. While the number of visits was similar in both groups throughout the training (Figure 3.40c), the rate of incorrect operant responses was significantly higher in the LV_sh_c-fos compared to the LV_sh_luc mice but, importantly, only when the rate of conditioned visits was high(Ono et al. 2006) (Figure 3.40b). LV_sh_c-fos animals also showed a faster rate of extinction, suggesting that the original memory trace was less consolidated.

Importantly, the behavioral deficits were not observed when the 'conditioned' tone was rare $(5 \%$ and $10 \%$, Figure $3.40 \mathrm{c}$ ). Indeed, since SSA was not affected by the manipulation, cortical responses to the conditioned tones, when rare, were presumably enhanced even after blocking c-Fos expression. Only when the 'safe' and 'conditioned' tones had similar probability, and deviance detection was not associated with the difference between the two, the behavioral deficits did 
emerge. Indeed, the late component of the auditory response, which was often present in LH_sh_cfos mice (Figure 3.39d), has been found to show deviance sensitivity(Chen et al. 2015).

In summary, for the first time we link a specific form of experience-driven plasticity in auditory cortex which depends on c-Fos expression with specific behavioral deficits. Our findings also show c-Fos involvement in learning and memory. Whereas for over 25 years c-Fos expression has repeatedly been shown to correlate with the synaptic plasticity, learning and memory, only the very recent studies have demonstrated, by means of optogenetics, that c-Fos expressing neurons form the memory engram (Tonegawa et al. 2015). Our experiments provide for the first time unequivocal support for the c-Fos function in neuronal plasticity, learning and memory. 
a

day of the training

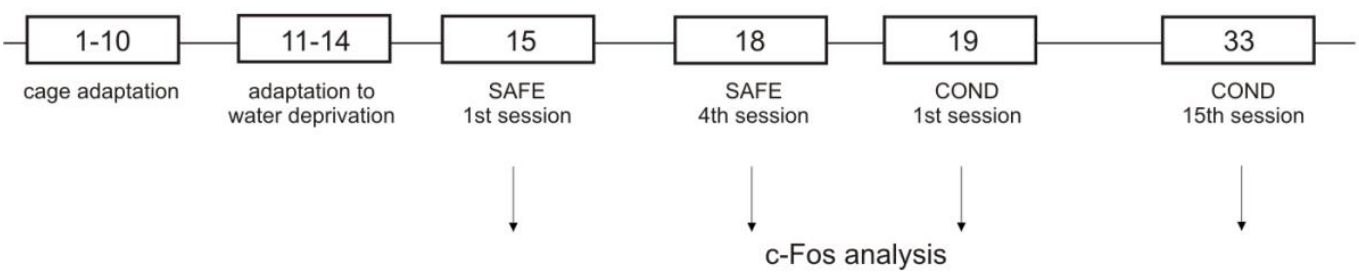

b

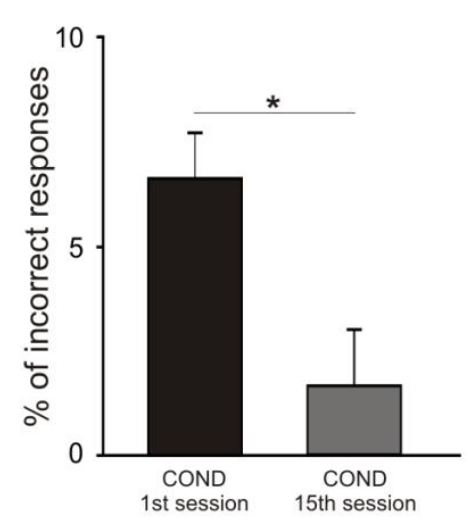

d

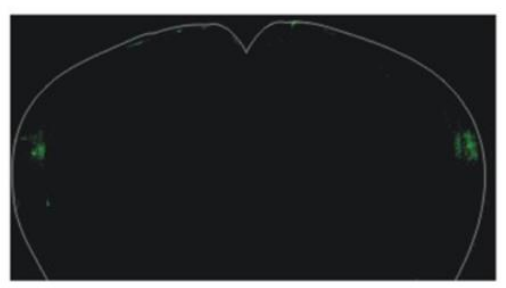

f

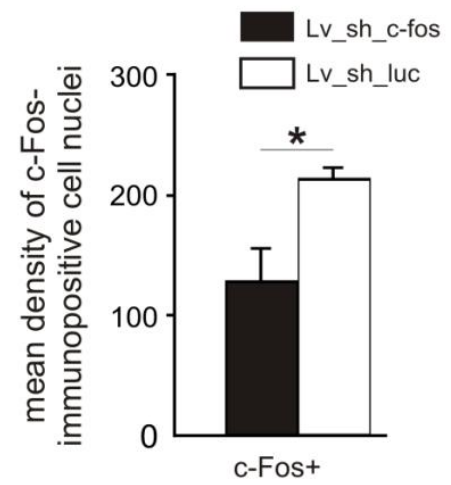

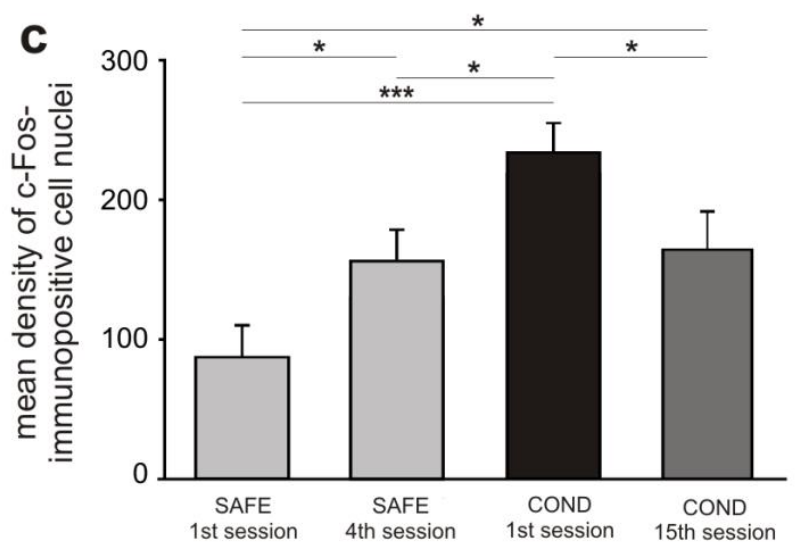

e
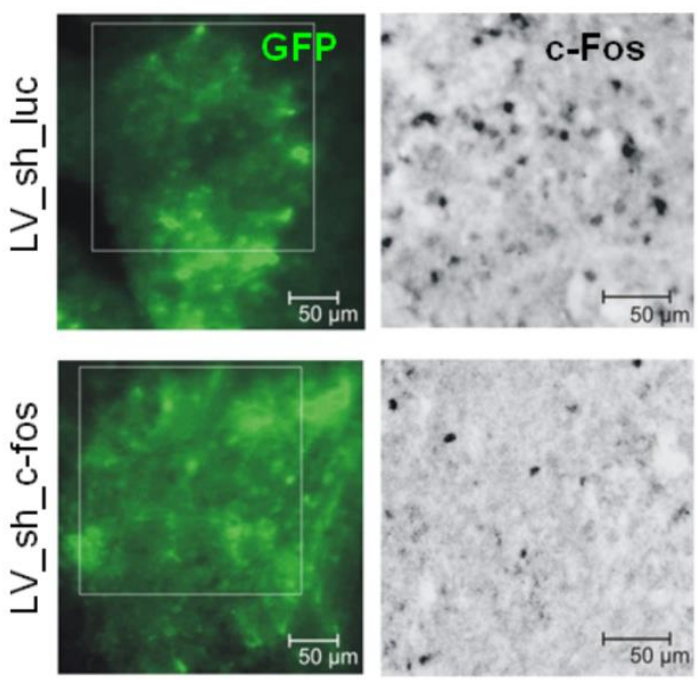

Figure 3.38: c-Fos expression increase in auditory cortex following sound discrimination training is blocked by lentivirally delivered shRNA against c-fos. (a) Sound discrimination training. After adaptation, a 'safe' sound $(6.7 \mathrm{kHz})$ accompanied every visit for 4 consecutive sessions. Then, an aversively 'conditioned' tone ( $13.4 \mathrm{kHz}$ ) was introduced in up to $17 \%$ of visits. (b) Rate of incorrect operant responses (nose-pokes during 'conditioned' visits) during the first and last sessions of discrimination training [Kolmogorov-Smirnov test]. (c) c-Fos expression in auditory cortex following repeated sound exposure: one-way ANOVA: $F(3,24)=8.10, p<0.001$, and Fisher's LSD test. (d) GFP signal in the lentivector infected area. (e,f) Sound-evoked c-Fos expression measured through cFos-positive cells within the infusion site [t-test], ${ }^{*} p<0.05,{ }^{* *} p<0.01,{ }^{* * *} p<0.001 ; \pm$ S.E.M. 

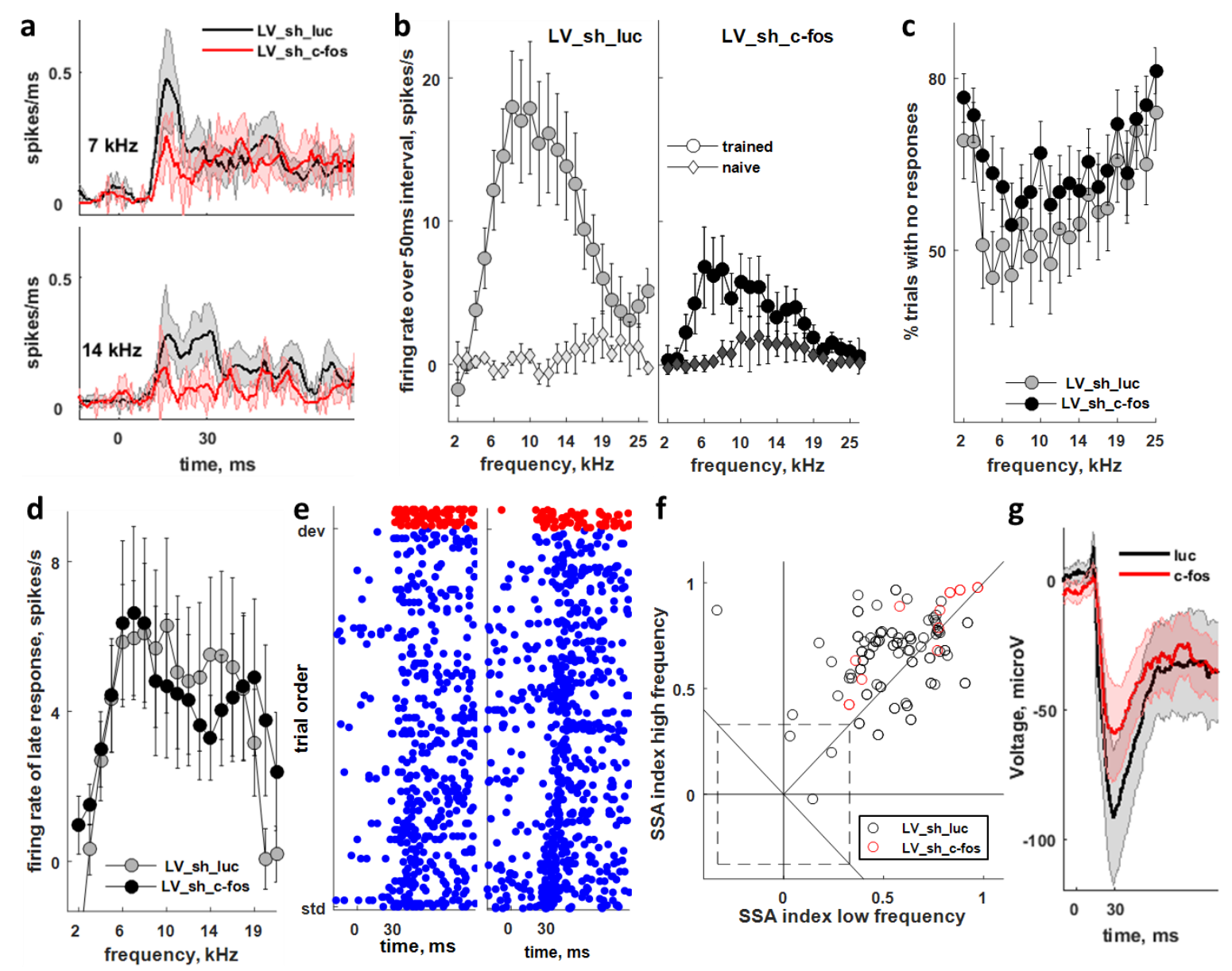

Figure 3.39: Blocking c-Fos expression in auditory cortex impairs experience-dependent plasticity. (a) Mean peri-stimulus time histograms evoked by the 'safe' (top) and 'conditioned' (bottom) tone in trained LV_sh_c-fos (red) and LV_sh_luc (black) mice. (b) Tuning (firing rate 1-50 ms after stimulus onset) in trained (circles) and naïve (diamonds) mice from LV_sh_c-fos (right) and LV_sh_luc (left) groups. We found a significant 3-way interaction $[F(21,5893)=4.51, p<0.0001]$ between the three fixed effects: group, training and frequency; as well as a group effect: $F(1,5893)=14.94, p<0.001$, a training effect: $F(1,5893)=9.22, p<0.01$ and a group $x$ training interaction: $F(1,5893)=6.37, p<0.05$. (c) Mean number of failures (trials without spikes) in evoked responses across tones for trained LV_sh_c-fos (black) and LV_sh_luc (gray) mice. (d) Tuning of late response (firing rate 50-200 ms after stimulus onset) for trained LV_sh_c-fos (black) and LV_sh_luc (gray) mice. (e) Example responses to a $9264 \mathrm{~Hz}$ tone when standard (blue) and when deviant (red) in a LV_sh_c-fos (left) and LV_sh_luc (right) mouse. (f) SSA indices for frequency-pairs presented in the oddball paradigm in trained LV_sh_c-fos (red) and LV_sh_luc (black) mice: $F(1,143)=2.11$, $\mathrm{p}=0.148$. (g) Mean local-field potential evoked by the 'conditioned' tone for LV_sh_c-fos (red) and LV_sh_luc (black) groups: $F(1,52)=1.3, p=0.259$ for the peak. 
a

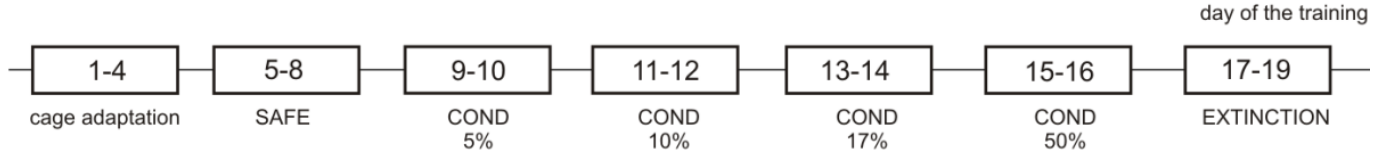

b

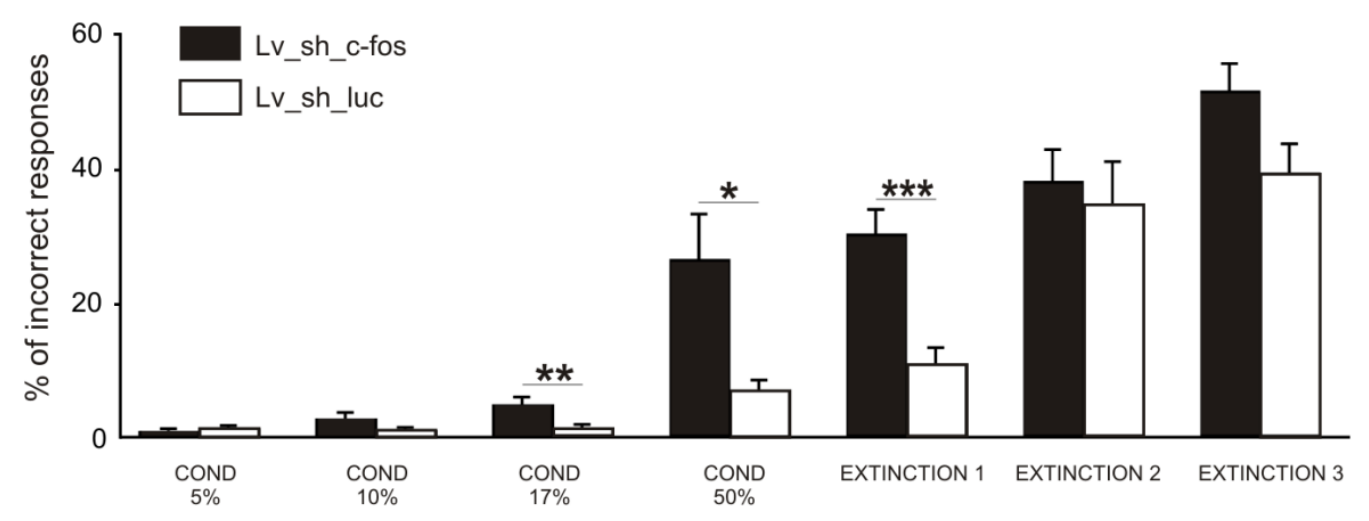

C

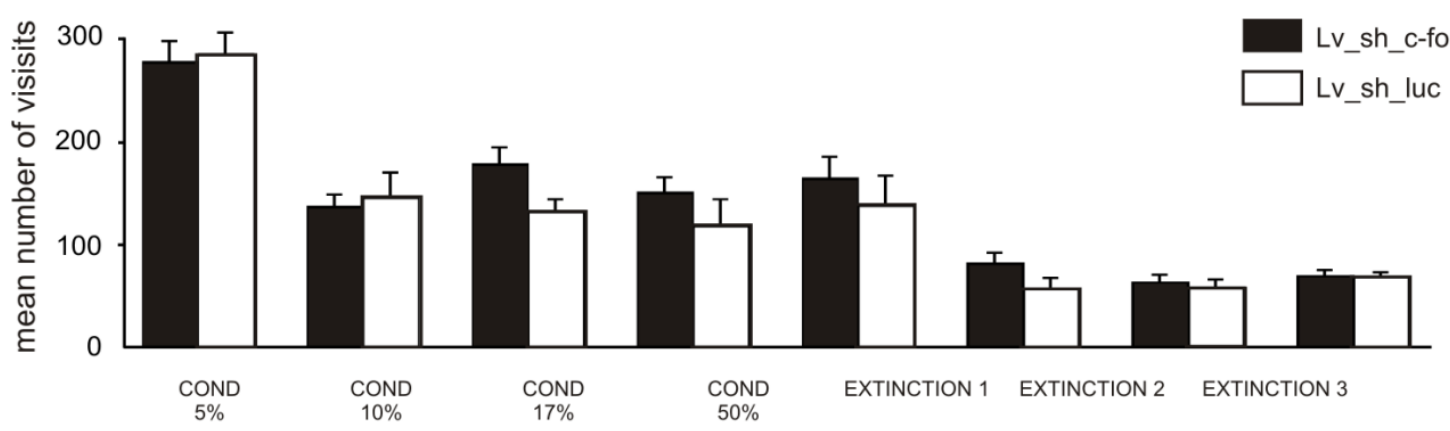

Figure 3.40. Blocking c-Fos expression in auditory cortex impairs sound discrimination learning. (a) Discrimination training scheme. 'Safe' and 'conditioned' phases were followed by three extinction sessions, during which nose-poke responses were not punished. (b) Incorrect responses during discrimination learning [two-way ANOVA (group $x$ session), the effects of group: $F(6,108)=9.87, p<0.01$, session: $F(6,108)=71.09, p<0.001)$, and the group $x$ session interaction: $F(6,108)=2.97, p<0.01)$; followed by one-way ANOVAs, dev17\% session: $F(1,18)=8.16, p<0.01$, dev50\% session: $F(1,18)=5.03, p<0.05$, and extD1 session: $F(1,18)=15.64, p<0.001]$. (c) Number of visits in the conditioning unit [Kolomogorow-Smirnow test]. 


\section{DISCUSSION}

Using a combination of behavioral, electrophysiological and molecular tools, in this work I have shown evidence of experience-dependence plasticity during adulthood in a subcortical nucleus in the auditory pathway- the inferior colliculus. Sound exposure that leaded to implicit auditory learning induced long term plasticity of sound processing in the IC that correlated with behavioral changes in frequency discrimination.

First, I found that at electrophysiological and behavioral level, the effects of sound exposure were dependent on the behavioral relevance of the exposed sound. Second, I found that behaviorallyrelevant sound exposure induced a functional reorganization in the IC as evidenced by: a reorganization of the tonotopic map preferentially tuned to higher frequencies, tuning curves with higher amplitude in the areas with a similar BF or lower of the exposed frequency, and smaller amplitude in the locations with a BF higher than the exposed frequency and, an expanded area that responded to the exposed sound. Third, I found that sound exposure also induced larger tuning curves in the lateral cortex of the IC. Fourth, while previous work on collicular plasticity has reached the conclusion that it depends on cortical input, I found that the maintenance of the collicular plasticity was not dependent of the cortico-collicular projections. Fifth, that the increase in the excitability and amplitude of the tuning curves, followed by relevant sound exposure, could be explained through a spatially localized increase in the ratio of E/I at the level of presynaptic molecules. Sixth, the reorganization observed in the IC was not due to an overall excitation in the auditory pathway, since no differences were found in the properties of the neuronal responses in the cochlear nucleus. Seventh, these electrophysiological changes paralleled behavioural changes such that relevant exposure decreased frequency discrimination acuity and elicit latent inhibition.

\subsection{Collicular plasticity due to sound exposure}

The main finding of this study is that sound exposure modifies sound processing in the IC in different ways that will be discussed separately in the following sections.

\subsubsection{Frequency representation}

Sound exposure induced a reorganization in frequency representation along the dorso-ventral and rostro-caudal axes in the IC of adult animals. The robust shift in frequency representation along the tonotopic axis, observed during the late phase of exposure, was partially "masked" during the early phase exposure due to a higher than normal frequency representation in control animals compared to those seen during the late phase. Collicular frequency representations of naïve animals reared in standard cages, were similar to those of the control animals during the late phase, indicating a 
transient change in frequency representation in the control animals. The transient change in frequency representation was paralleled by a decrease in evoked activity, as discussed below. This finding supports the hypothesis that the IC is under constant adaptation to the acoustic environment. In the exposed animals, there was a shift towards high frequencies from the early to the late phase. The shift in frequency representation was evident when animals were exposed to $16 \mathrm{kHz}$. In my experiments, the BF of animals exposed to $8 \mathrm{kHz}$ showed a tendency to be higher than control, however the dispersion of the control animal was high and did not reach significance. It is plausible that frequencies lower than $16 \mathrm{kHz}$ might induce shifts in frequency representation. There is evidence that in mice, electrical stimulation of the $A C$ shifts the BF of collicular neurons by $12 \pm \mathrm{kHz}$ to match the cortical BF (egocentric cortical feedback) (Yan \& Ehret 2001; Yan \& Ehret 2002). However, it is not known whether this range of frequency adjustment applies to all the collicular neurons, or only to neurons within a specific range of BF. The mechanism of egocentric cortical feedback seems plausible to adjust frequency specificity in the IC to relevant acoustic input. However, I did not observe an egocentric adjustment towards the exposed sound but always towards high frequencies. This occurred even at ventral depths with higher BF than $16 \mathrm{kHz}$. In fact this shift seemed to appear earlier than the shift at lower frequencies. The shift in the frequency representation, could also indicate an expansion in the width of the frequency lamina tuned to 16 $\mathrm{kHz}$ that could be the equivalent of cortical expansion to specific sounds seen in the $\mathrm{AC}$ by others (Weinberger 2004; Bao 2015). In support of this idea, the analysis of the simultaneous responses to $16 \mathrm{kHz}$ along the dorso-ventral axis showed that the amount of the response area was expanded $\sim 100 \mu \mathrm{m}$ more in the exposed group. This expansion could also explain the dorsal shift of the frequency laminas along the rostro-caudal axis. Interestingly, the size of the area that responded to $8 \mathrm{kHz}$ was the same as the control group, suggesting a frequency specific expansion. However, an expansion of the frequency lamina does not explain the shift to high BF of locations with higher BF than the exposed and requires further characterization, for example, to determine if the shift also occurs in locations with a BF further apart from $16 \mathrm{kHz}$ (e.g. $>32 \mathrm{kHz}$ ).

The only report that explored the effect of non-traumatic sound exposure, during adulthood, on sound representations in the IC did not find any differences in the distribution of BFs (Bureš et al. 2014). These discrepancies could be due to different reasons: 1) a rippled noise was used as the exposed sound, which made it difficult to evaluate for specific changes in the tonotopy; 2 ) they did not report a systematic approach in the insertion of the multi-electrode array, without which there could be a high degree of variability. In contrast, in this study pure tones were used with the intention to assess frequency-specific effects, and measurements from the medio-lateral and 
rostro-caudal borders were always made to assure the insertion of the electrode always in the same central portion of the IC.

It has been shown that passive exposure during the auditory critical period can lead to an overrepresentation of the exposed tone in the majority of the studies (Poon \& Chen 1992; Yu et al. 2007; Oliver et al. 2011; Miyakawa et al. 2013). In most of the studies, frequencies of $14 \mathrm{kHz}$ or above have been used. Using a similar rearing paradigm, but $7.5 \mathrm{kHz}$ as the exposed sound, Miyakawa et al. (2013) reported a transient narrowing of the tuning specific to the exposed tone, but no changes in the tonotopy of the IC. Interestingly, both rearing paradigms have been shown to generate an overrepresentation of the exposed tone in the auditory cortex (Zhang et al. 2001; Han et al. 2007). This discrepancy in the results between studies is in agreement with the theory that exposure to frequencies only above $14 \mathrm{kHz}$ can induce a robust change in collicular tonotopy. My results suggest that sound exposure during adulthood induces and expansion of the anatomical frequency lamina in the IC of adult animals, that leads to a reorganization of the frequency representation. This happens as a possible mechanism of adaptation to behaviorally relevant sounds. It is possible that the expansion of the frequency lamina results from the combination of an enriched environment as the Audiobox and the relevance of the acoustic input. Although random exposure to sound also induced a shift in frequency representation, it was lower in magnitude, indicating and additive effect of behavioral relevance to frequency reorganization in the IC. The reorganization in frequency representation in the IC of the exposed animals could serve as a mechanism of adaptation that generalize the value of the experience sound to include similar sounds (categorize), as a way to filter information in an efficient way.

\subsubsection{Tuning curves and temporal firing patterns}

Sound exposure also induced changes in the amplitude of the tuning curves at different depths of the IC. During the early phase of exposure, the evoked activity of the control group was larger than that of the exposed group. However, the activity dropped until it was smaller than the exposed group. Together with the transient shifts in frequency representation it strongly suggests that the environment of the Audiobox itself can induce plasticity. It has been shown that a short period of exposure to an enriched environment enhances plasticity in the barrel cortex (Rema et al. 2006). It is plausible then that the environment of the Audiobox triggers a transient state of enhanced plasticity in the brain that is capitalized differently between the exposed and control group. For the control group, the lack of a patterned auditory input in the corner that lead to implicit learning, as occurred with the exposed group, could be the cause that leaded to a downregulation in the high collicular evoked activity observed during the first days. This downregulation could be achieved by 
different mechanisms of homeostatic plasticity, such as the internalization of glutamate receptors (Sale et al. 2014; Nithianantharajah \& Hannan 2006). Enriched environment has also been shown to cause remodeling of synaptic spines (Bednarek \& Caroni 2011). Additionally, the turnover of spines can be directly regulated by sensory experience (Holtmaat et al. 2005), and novel sensory experience can promote stabilization (Holtmaat et al. 2006). It is possible that the Audiobox triggers spine remodeling that in the exposed group is stabilized and strengthen by sound exposure and the development of an association through implicit learning.

The non-specific increase in the amplitude of the tuning curves, in areas non-tuned to the exposed sound could be a reflection of the expansion of the frequency lamina that was specific to the exposed group. Interestingly, the random group showed a different pattern in evoked activity, namely a robust increase in activity in the putative dorsal cortex, that correlated with an increase in the area that responded to low frequencies such as $8 \mathrm{kHz}$. This effect was not seen in the exposed group. Although the tuning curves of the area tuned to $16 \mathrm{kHz}$ were also increased in the random group, the area that responded at to $16 \mathrm{kHz}$ was similar $(\sim 250 \mu \mathrm{m})$ to the control group. These two contrasting results between the exposed and the random group, in the amplitude of the responses as well as the area devoted to 8 and $16 \mathrm{kHz}$, indicate that the plasticity induced by the different paradigms of sound exposure is not the same. Furthermore, it suggests that the IC adjust its spectral tuning accordingly to the relevance of the sound and that is susceptible of implicit auditory learning. These differences were directly reflected at a behavioral level (Figure 4.1, as will be discussed in detail later).

Not only was the evoked activity increased in the exposed group but also the spontaneous activity, which reflected the overall excitability of the IC. I did not observe changes in the thresholds and bandwidth of the tuning curves as has been reported for the auditory cortex in animals that lived in an enriched environment (Engineer 2004). My results are in agreement with Bures et al. (2014) where they found that 2 weeks AAE in adult rats increase evoked responses and spontaneous activity of neurons in the IC regardless of its BF. In contrast, they found sharpened tuning curves and lower threshold in neurons with $\mathrm{BF}>8 \mathrm{kHz}$. In that study the authors analyzed the activity of single units while here my analysis was based on multiunit activity. It is possible that in the analysis of evoked activity from a small population of neurons some differences are diluted due to the contribution of neurons with heterogeneous properties. In that respect, it has been shown at least four types of neurons with different properties in bandwidths and thresholds the IC (Egorova et al. 2001). 
Recently, it has been shown that there is a rapid change in the spectral selectivity of neurons in the IC following engagement in an auditory detection task in awake ferrets (Slee \& David 2015). This changes were similar to those previously observed in the AC (David et al. 2012). The PSTHs of neurons with BF matching that of the target tone were suppressed while neurons with nonmatched BFs showed less suppression or an enhancement of the responses. More interestingly, the plasticity observed during the task lasted after the end of the behavior in the majority of the recorded neurons. I could not detect such differences in my experiments, most likely because the animals were anesthetized. Anesthesia is a factor that could masks potentially rapid plasticity and the influence of possible online modulators in the responses to sound. Nevertheless measurements of plasticity can still be performed with reliability (Polley et al. 2006; Han et al. 2007; Guo et al. 2012; Zhou \& Merzenich 2012). Awake recordings in mice using a similar paradigm as the Audiobox would be required to analyze online changes in the spectral tuning of collicular neurons due to sound exposure.

What is the functional role of a change in the amplitude of a tuning curve? Neurons in the IC are tuned to frequencies with a particular preferred frequency. It is logic to think that these neurons will convey maximal information at their BF (maximum firing rate). However, the dynamic range of spike rate is not located in the BF but in the flanks of the tuning curve where they have a steepest slope can also offer a good source of information, since small changes in frequency would produce larger differences in spike rate. In a theoretical study, it has been shown that when the trial-to-trial variability is high, the maximum specific-information is carried at the peak of the tuning curve (Butts \& Goldman 2006). When trial-to-trial variability is reduced, the maximal information is carried in the flanks of the tuning curves. For neurons in the auditory cortex the maximal information is at the BF (Montgomery \& Wehr 2010), assuming that the amount frequency information is encoded in spike rates. If it is the case for the IC, then an increase in the amplitude of the tuning curve would not make such big difference if the BF remained the same. However, if the maximum information is carried at the flanks of the tuning curve, an increase in the amplitude of the tuning curve would generate an increase in the amount of information carried. In this case, tuning curve amplitude between the exposed and random groups was similar in the collicular areas tuned to $16 \mathrm{kHz}$ while frequency representation was different along the tonotopic axis. Since the behavioral experiments showed a difference in frequency discrimination between these two groups, it strongly suggests that is the $\mathrm{BF}$ and not amplitude of the tuning curve the relevant parameter with the maximal information at the level of the IC. Further theoretical studies will be required to corroborate this hypothesis.

\subsubsection{Lateral cortex}


The LC receives inputs from auditory and non-auditory areas (Aitkin et al. 1978; Oliver 2005). Interestingly, the electrophysiological properties of neurons in the LC have been poorly studied. Compared to the ICC, in our recordings tuning curves of LC were broader in agreement with previous studies (Syka et al. 2000). Tonotopic input has been reported for the LC of cats and mice (Loftus et al. 2008; Stiebler \& Ehret 1985) based on anterograde tracers injected in the cochlear nucleus and electrophysiological recordings. I did not observe a particular tonotopic arrangement, probably due to the orientation the recording electrode. To my knowledge this is the first study to address plastic changes in the lateral cortex of the IC. As with the recording in the central IC, I found that the activity during the early phase of exposure was larger for control and exposed groups than during the late phase of exposure. These results add evidence to the initial increase in overall excitability in the whole IC, caused by the environment of the Audiobox. Nevertheless, in contrast to the recordings in the central IC, the evoked activity in the LC was stronger in the exposed group already in the early phase of exposure. This result suggests that multisensory neurons in the LC are more susceptible to undergo plastic changes. In support of this idea, analysis in slices revealed that neurons in the LC have a lower threshold for firing than those in central IC, therefore they are more excitable (Ahuja \& Wu 2007). Additionally, the LC sends projections to the ipsilateral central IC (Chernock et al. 2004), suggesting the possible role of multisensory neurons in the IC as a key initiators of collicular plasticity. Furthermore, the LC projects to the medial division of the geniculate body, who has a modulatory role in the input that reaches the auditory and somatosensory cortices (Edeline \& Weinberger 1992). It has also been implicated in the circuits that modulate auditory fear conditioning (LeDoux et al. 1985). A more detailed study will be necessary to address the specific role of the LC in collicular plasticity and its modulation by other sensory modality.

\subsubsection{Overall excitability in the auditory pathway}

I found several changes that pointed to an increase in the excitability of the IC: a transient increase in evoked activity during the early phase of exposure for control and exposed group and larger amplitude of tuning curves and higher spontaneous activity followed by sound exposure during the late phase. Interestingly, the increase in excitability did not seem to be a generalized state in the auditory pathway. Recordings in the cochlear nucleus, during the late phase of exposure, revealed that sound exposure does not alter sound processing at that level. Nevertheless, the cochlear nucleus is also susceptible of cortical influence that can modify its activity (Kong et al. 2014; Luo et al. 2008) as well as certain forms of plasticity such as short-term and spike-time dependent plasticity (Tzounopoulos et al. 2004; Wang et al. 2011). Recordings during the early phase of exposure were not made to establish whether the initial excitability observed in both groups also holds true for sub-collicular nuclei. 


\subsection{Corticofugal projections and frequency representation}

The finding that cortical inactivation during acute recordings did not modify the changes observed between control and exposed groups during the late phase, suggests that at least once the collicular plasticity is established cortical control is not required. The effects observed after cortical inactivation in the neuronal responses were heterogeneous, but the finding that its effect was larger during the early phase suggests a dynamic influence of the cortico-collicular projections. Heterogeneity of effects in neuronal responses due to $A C$ silencing has been previously reported. Popelář et al. (2003) showed heterogeneous changes in firing rates of collicular neurons after AC inactivation with TTX while thresholds and tuning properties were unchanged. These heterogeneous results suggest that cortico-collicular projections might target different circuits in the IC. It has been reported that electrical stimulation of the AC leads to transient changes in the collicular frequency representation of bats and mice that depends on the cortical BF of the area electrically stimulated such as collicular frequency representation is centered around the stimulated cortical BF (Yan \& Ehret 2001; Gao \& Suga 2000; Suga et al. 2002; Suga \& Ma 2003). Since reports of changes in collicular frequency representation have always been accompanied by changes of the CC projections and the view that IC plasticity depends on cortical plasticity is well extended, we hypothesized that the shift in frequency representation observed in relevant-exposed animals was dependent on CC projections. Since cortical inactivation did not alter the frequency shift observed in our experiments, it suggests that $\mathrm{CC}$ projections are not required for the maintenance of this change.

\subsection{Underlying mechanisms}

The main finding of the gene expression experiments is that control and relevant exposed animals showed a similar pattern of expression in the genes measured, suggesting that it is the Audiobox and not the sound exposure the main factor responsible for these changes. In the IC the expression of the genes measured in this study has been evaluated only in the acoustic-trauma and hearing loss context (Dong et al. 2010; Holt et al. 2005; Hu et al. 2014; Marianowski et al. 2000; Tan et al. 2007;) making a direct comparison difficult. The gene expression analysis of this work was made to have a general overview of the molecular changes that might occur following relevant sound exposure in the IC. Since neuronal function is tightly regulated at different levels (from gene expression to post-translational modifications of proteins), an increase in gene expression does not necessarily corresponds with an increase in protein or a modification in synaptic function (Sutton \& Schuman 2006). However, gene expression analysis are more straightforward to perform than other assays that measure general protein levels, such as western blots, or more specific such as 
immunohistochemistry analysis, and can offer a faster overview of the possible molecular changes that might be happening following relevant sound exposure. Additionally, the electrophysiology and the gene expression experiments can complement each other to offer a better understanding of adult collicular plasticity, so far poorly understood.

From the genes measured, the most robust increased was in CREB. CREB is a transcription factor involved in long term neuronal plasticity (Tao et al. 1998; Lee et al. 2013; Tardito et al. 2006). For example, it has been shown that whisker use in rodents induces the expression of CREB and BDNF (Gomez-Pinilla et al. 2011). BDNF is a neurotrophic factor known to be involved in multiple functions such as neuronal survival, axonal and dendritic growth, synapse formation and synaptic plasticity and, together with cfos, is a direct target gene of CREB (Martinowich \& Lu 2008). It has been shown an increase in the cortical expression of BDNF followed by enriched environment and sensory experience (Falkenberg et al. 1992; Zhu et al. 2014). In this study I found a reduction of BDNF and no change in cfos expression. How an increase in expression of CREB leads to a reduction of BDNF? In order to activate gene transcription, CREB needs to be in its phosphorylated form (pCREB). The levels of PCREB has been used a signature of transcriptional activation (Gonzalez \& Montminy 1989), therefore it is possible that there could be no differences in the levels of collicular pCREB and consequently no transcription of the BDNF gene. Here I did not measure protein levels of pCREB that could have shown a more accurate measure of CREB activity. However, it has been shown positive correlations of gene expression between CREB and BDNF upon different conditions (Chaudhury \& Wadhwa 2009; Nibuya et al. 1996). Another explanation for the low mRNA levels of BDNF would be an increase in the translation rate that leads to a reduction in mRNA levels. Translational control has been shown to be an important regulatory phenomenon in neuronal plasticity although it has not been demonstrated for BDNF (Belelovsky et al. 2005; Gal-Ben-Ari et al. 2012; Sutton \& Schuman 2006). Protein level measurement of BDNF would help to clarify this point.

The finding that the expression of the subunits 1 and 2 of the AMPA receptor was specifically reduced in the control group might indicate a general downregulation of the AMPA mediated synaptic transmission that might be directly reflected in the smaller tuning curves of the control group during the late phase. Interestingly, I found an increase in the ratio between the expression of the presynaptic molecules Vglut2/VGAT, for control and exposed groups. A more detailed immunohistochemical analysis of the protein levels showed an increase in the $E / I$ ratio of the sound exposed group over the control only in the dorsal part of the IC, this increase was mainly due to a reduction of VGAT expression that we could not detect in the gene expression analysis suggesting that pre-synaptic plasticity occurs in a particular location of the IC. The ratio between Vglut/VGAT 
has been used to express the $\mathrm{E} / \mathrm{I}$ at the presynaptic level and as a signature of pre-synaptic plasticity following environmental manipulations (Mainardi et al. 2014; Mainardi et al. 2010). By measuring Vglut2 levels, we cover the most abundant form of Vglut in the IC. It has been shown to be located in brainstem nuclei that input into IC as well as in local IC terminals, while terminals that contain only Vglut1 are most likely to arise from pyramidal neurons in the layer V of AC (Ito et al. 2009; Winer et al. 1998). A measurement of Vglut1 expression would help to determine whether corticocollicular pre-synaptic plasticity also occurs followed by sound exposure. Interestingly, I found a decrease in the expression of GAD in auditory cortex, suggesting a reduction in inhibition due to relevant sound exposure at the cortical level. These finding is in agreement with previous reports where a regulation of cortical GAD has been observed following sensory learning (Gierdalski 2001; Donato et al. 2013).

The increase in the $\mathrm{E} / \mathrm{I}$ ratio suggest an increase in the release probability at collicular synapses (Takamori 2006; Wojcik et al. 2004), thus providing a plausible mechanism that explains the increase in spontaneous activity, and the larger amplitude in the tuning curves observed in relevant exposed animals. Indeed, it has been shown that synaptic inhibition mediates ocular dominance plasticity (Ma et al. 2013; Hensch et al. 1998; Hensch 2014) and many studies have shown the importance of the $\mathrm{E} / \mathrm{I}$ balance in shaping the neuronal tuning properties in the auditory cortex $\mathrm{Wu}$ et al. 2008; Wehr \& Zador 2003; Benali et al. 2008; Seybold et al. 2012; Rubenstein \& Merzenich 2003). Specifically in the IC, it has been described that GABA mediated synaptic transmission shapes receptive fields and frequency tuning curves differently in two populations of IC neurons (Vater et al. 1992; Yang et al. 1992; Faingold et al. 1991; LeBeau et al. 2001). In the first group, GABAa receptor blockade generates an increase in the firing rates only in the excitatory response of the receptive field with little change in the firing lateral to the tuning curve. In the second group, GABAa receptor blockade increased the firing rates at the $\mathrm{BF}$, similar to the previous group, but it also broaden the frequency range of the original tuning curve for that neuron increasing thus the bandwidth of the tuning curve (Yang et al. 1992; LeBeau et al. 2001). Based on the finding here that sound exposure induced tuning curves with larger amplitude but no apparent increase in bandwidth, one can speculate that the reduction in inhibition occurs at a particular type of neurons that become more responsive to acoustic stimulation. A more detailed characterization of these two types of neurons would be important to determine whether one particular type of neuron in the IC is more susceptible to plastic changes than others.

\subsection{Changes in frequency discrimination due to sound exposure}


Interestingly, the collicular plasticity induced by relevant sound exposure correlated with behavioral changes: mainly a decrease in frequency discrimination acuity (FDA) as measured by the inhibition of the acoustic startle reflex (ASR), induced by pre-pulses of different frequencies from the background tone (PPI). PPI has the advantage that does not require learning, making it a useful behavioral test to measure changes in frequency discrimination acuity after different paradigms of acoustic experience, such as acoustic fear conditioning or acoustic trauma (Clause et al. 2011; Aizenberg \& Geffen 2013b; Mwilambwe-Tshilobo et al. 2015). Aizenberg \& Geffen (2013) have shown that FDA can be modified after auditory fear conditioning depending on whether the animals generalized the fear response to a wider range of tones (low-specificity), or whether the fear response is expressed only in frequencies very similar to the conditioned tone (high-specificity). While low-specificity decreased FDA (that explains the generalization of the fear response to other non-conditioned tones) higher specificity increased FDA. Here, it is possible that exposed animals generalized to other tones the behavioral meaning of the exposed tone (as a safe signal), that leads to a decrease in FDA. This generalization might also explain the generalization of latent inhibition to other frequencies found by de Hoz \& Nelken (2014). While the Aizenberg study showed an average decrease of the FDA for non-specific learners of $100 \%$, in our case the decrease in FDA was around $86 \%$. It is important to note that in this study, FDA comparisons came from different groups of animals and not within the same animals before and after sound exposure as in the Aizenberg study. Nevertheless, the finding that specific sound exposure in the Audiobox decreased FDA suggests that its behavioral relevance is capable of modifying behavior close to auditory fear conditioning. PPI is present in animals decerebrated at the level of the reticular formation leaving intact the IC intact, indicating that the main circuits are located at the midbrain and brainstem level (Fox 1979). Furthermore, lesions of the IC have shown to reduce PPI levels, suggesting that the IC is an important relay in the PPI circuit (Fox 1979; Li et al. 1998), while cortical lesions had no effect (Li et al. 1998). It has been proposed that the role of the IC in the expression of PPI is to send auditory information to the superior colliculus via the lateral cortex. The superior colliculus, sends projections to the pedunculopontine tegmental nucleus (PPTg) that in turn inhibits the caudal pontine reticular nucleus (PnC) via an inhibitory cholinergic projection (Koch 1999). The PnC has been shown to be critical part of the ASR, since it projects to spinal, facial, and cranial motor neurons (Koch 1999). Recently, it has been shown that parvalbumin positive neurons in the AC modulate frequency discrimination acuity (Aizenberg et al. 2015). Modulatory cortical information can be transferred to the lateral cortex of the IC via cortico-collicular projections (Winer 2005). Interestingly, apomorphine injections in the IC reduce PPI (Satake et al. 2012), suggesting a dopaminergic control in the filtering of auditory information at the IC level. It is probable that this adjustment requires neuromodulators such as dopamine. Further experiments that prevent 
collicular plasticity due to sound exposure, by pharmacological or optogenetic means, will be needed to test this theory.

The frequency laminas in the IC have been correlated with perceptual critical bands, which are a range of frequencies perceived with the same pitch, and has been proposed as the place where perceptual critical bands arise. For a given frequency lamina the frequency range covered is the same as the perceived critical band: 0.3 octaves. Also, the distance in frequency range between one frequency lamina to the other is the bandwidth of a critical band (Ehret \& Schreiner 1997; Schreiner et al. 1997; Ehret \& Schreiner 2005). The perception of sounds related with the critical bands includes the perception of tones in a noisy background and changes in the identification of vowels by shifts of frequency components (Ehret \& Schreiner 2005). In the PPI experiments, a pre-pulse sound needs to be distinguished from the background tone to inhibit the ASR, and it might be directly related with critical bands. The increase in FDA of the exposed group might indicate that frequency resolution is altered in the spectral coding in the frequency laminas of the IC. This alteration could be the expansion in the responsive area to the exposed sound or the shift in BF to high frequencies. This last correlation is plotted in the Figure 4.1B, where it can be appreciated that frequency discrimination threshold increases with increasing frequency shift in the IC. This finding strongly supports the theory of the IC as a subcortical filter of current auditory information that is adjusted by implicit auditory learning

\subsection{Sound exposure and latent inhibition}

The main finding of this set of experiments is that random exposure during the pre-conditioned phase to the future conditioned sound is not enough to generate $\mathrm{LI}$, since animals could discriminate well between the safe and conditioned sound (an average $d^{\prime}$ around 1 ) already in the first day of conditioning. In this experiments, animals from the $\mathrm{LI}$ and random exposed groups did not reach the same high levels of visits without nose-pokes during conditioned visits compared to control animals ( $75 \%$ vs $90 \%$, respectively) as has been shown in previous experiments (de Hoz \& Nelken 2014). Also, during the conditioning phase, the percentage of visits without nose-pokes during the safe visits of the control animals did not return to pre-conditioned levels as in the previous study. These differences could arise from differences in the experimental settings. The probability used for conditioned visits in the de Hoz \& Nelken (2014) study was $17 \%$ while in this study was $29 \%$. A higher probability of conditioned visits could generate a more conservative approach for the control group that prevented them nose-poked during the safe visits. Nevertheless, these differences do not invalidate these results since the discriminability indexes 
calculated are in agreement with the previous report. However, a replication using the same probability of conditioned visits will be required.

One theory states that $\mathrm{LI}$ is the expression of the competition between one association previously acquired and a new association to the same sensory stimulus. This explanation is supported by our results where only animals that implicitly learned the association sound-corner in the Audiobox (relevant exposed animals) but not random exposure elicited $\mathrm{LI}$, and by experiments that have shown context-specificity of $\mathrm{LI}$ where non-competitive associations are created (Lovibond et al. 1984; Honey \& Good 1993; Diaz et al. 2015). Here the expression of latent inhibition and the change in frequency representation seems also to be related (Figure 4.1A). This could be due to the modified spectral processing that the implicit learning elicits in the LI group to the conditioned tone during the preconditioned phase.

The IC has been implicated as an important structure in the expression of $\mathrm{LI}$. It has been shown that local injections in the IC of the dopaminergic agonist apomorphine suppress the expression of $\mathrm{LI}$ in rats (Melo et al. 2009). Dopamine is known to modulate firing rates, spontaneous activity and
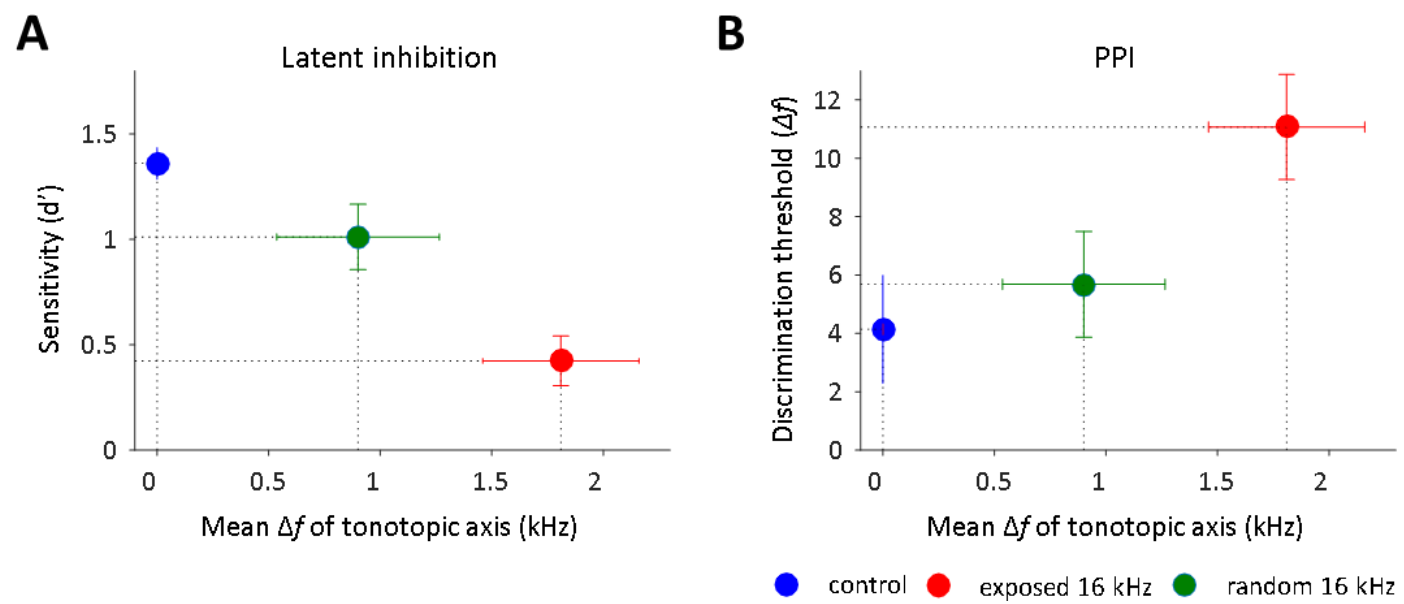

Figure 4.1: Correlation between two behavioral measures of frequency discrimination and the shift in the tonotopic map caused by relevant and random tone exposure. A, Average $d^{\prime}$ values during the first day of conditioning in the latent inhibition paradigm and $\mathbf{B}$, average discrimination threshold of the PPI test, both, as a function of the averaged frequency shift along the tonotopic axis of the IC in control, exposed and random exposed animals.

latencies in an heterogeneous manner of neurons in the IC (Gittelman et al. 2013). Considering the important role of dopamine in reward signaling as well as to modulate plasticity in auditory cortex (Bao et al. 2001; Bao et al. 2003; Bermudez \& Schultz 2014; Schultz et al. 2015), it is possible that dopamine plays an important role in the collicular plasticity involved in the expression of LI. Further 
experiments involving pharmacological or pharmacogenetical manipulations in combination with acute electrophysiology will be required to address this question.

\subsubsection{Collicular plasticity due to conditioning}

The finding that animals in the $\mathrm{LI}$ group and the random exposed group showed different frequency representations and neuronal responses in the IC at a time when they already have similar discriminability indexes during the conditioned phase suggests that sound exposure during the preconditioned phase might still influence future plasticity. It has been shown that visual experience leads to long lasting structural signatures in the form of persistent dendritic spines in visual cortex, that in the event of new experience did not disappear but increase in size (Hofer et al. 2009). Similar anatomical mechanisms could account for the differences observed between the groups that directly affect frequency representation and processing. If that is the case, I would expect the control conditioned animals to also show different frequency representations. Further experiments with recordings from the control conditioned group would be required to answer this question. Additionally, experiments that would allow for chronic imaging of dendritic spines would be also useful to determine the dynamic of structural changes, if any, in the IC following implicit learning and subsequent conditioning. Compared to $\mathrm{LI}$ and control untrained animals, the random exposed animals showed a shift in frequency representation towards lower frequencies while LI animals had a similar frequency representation to control untrained animals. This could suggest for the random group a possible egocentric reorganization towards the safe tone in the rostral-half of the IC, and an egocentric reorganization towards the conditioned tone in the caudal half of the IC. This shift in frequency representation correlated with stronger responses to the safe tone in the rostral part and to the conditioned tone in the caudal part. It has been shown that the pattern of responses to the same frequency changes according to the location in the frequency lamina. For example, the rostral portion of the same frequency lamina responds to the same stimulus with more spikes, but for a shorter time than the caudal portion of the same lamina (Lim \& Anderson 2007b; Straka et al. 2014). These differences arise due to the different patterns of synaptic inputs that arrive to the same frequency lamina from sub-collicular structures (Loftus et al. 2010; Ono \& Ito 2015). It is possible that the segregation in the locations of the strongest responses to the safe and conditioned frequencies (rostral vs caudal) in the random group reflects the functional role of the different pattern of synaptic inputs within a frequency lamina, that might encode for the behavioral value of the auditory information. Why the same pattern was not seen in the Ll group is still an intriguing question. Recordings during the pre-conditioned phase will be required to answer whether the segregation, or lack of it, in the pattern of responses to the safe and conditioned tones arose previously to conditioning and directly determines the ability of the animal to discriminate between 
these tones, or whether it is the consequence of integration between previous and new relevant experience. These results indicate that the IC "accumulates" acoustic experiences in a continuous way. This accumulation influences how the new experience will be processed and, importantly, how the new experiences will be imprinted in the IC (Skoe \& Chandrasekaran 2014).

\subsection{Experimental considerations}

C57BL/6J animals suffer from a progressive degeneration of inner and outer hair cells starting at the base of the cochlea, leading to high frequency $(>30 \mathrm{kHz}$ ) hearing loss by P80 (Hequembourg \& Liberman 2001). I used young animals that were around P37 when they were placed the Audiobox and P40-52 when the acute recordings were made, made comparisons between animals of the same age, and used frequencies below $20 \mathrm{kHz}$. Thus, our experiments were not influenced by the congenital deficit in C57BJ/6J mice. This age was beyond the auditory critical period described for mice (P11-21) (Barkat, Polley, \& Hensch, 2011). Recently, age-related behavioral and synaptic alterations have been described in auditory cortex of animals during early adolescence (P28) compared to young adult animals, while no changes were found between young adult and late adolescent mice (P56) (Moyer et al. 2015). Although I used young animals in this study, the age at the time of experiment does not coincide with the important time points of auditory plasticity (P1128) previously described.

\subsection{Conclusions}

The IC is the first structure in the auditory pathway where all auditory nuclei converge. This feature makes it an ideal candidate to be the first site in the pathway where previous experience in the form of implicit auditory learning influences the processing of auditory information. The goal of this study was to test this idea by measuring plastic changes in the IC of mice that went through specific auditory experience. The results of the present study show that the IC in adult mammals undergoes important plastic changes that affect sound processing. These plastic changes include a wide range of effects observed at an electrophysiological and molecular level. The main changes are an increase in excitability, an increase in evoked responses and a shift in frequency representation. At the molecular level, the main change is a decrease in inhibitory presynaptic markers. Additionally, the shift in frequency representation correlates with a decrease in frequency discrimination at a behavioral level, thus strongly supporting the idea of the IC as a key player in filtering auditory information. Therefore it is plausible that the neuronal substrate for implicit auditory learning includes the neurons in the IC. Not only the IC filters auditory information but it also seems to accumulate acoustic experiences to, again, influence the processing of incoming auditory information. This study has important implications to place the IC in a central and active role in the 
field of auditory plasticity. So far, studies related with auditory experience-dependent plasticity have been centered on the AC. It is becoming clear now that the AC does not "act" alone in the filtering of auditory information, but rather this process involves active feedback loops between multiple structures including neuromodulatory nuclei. With the new technologies that allow the study of specific circuits with precise timescales, it will facilitate the study of auditory processing in a more integrative view of highly dynamic neuronal circuits rather than individual structures.

Implicit auditory learning has been related to the developing of important communication processes such as the categorization of phonemes (Gabay et al. 2015). The acquisition of novel auditory non-speech categories through implicit learning is impaired in dyslexic patients, suggesting that dyslexic patients have a general deficit in auditory category learning that result in the negative effects of language acquisition and reading (Gabay \& Holt 2015). Furthermore, by measuring auditory brainstem responses it has been shown that children with developmental dyslexia show an impairment in context-dependent encoding of speech (Chandrasekaran et al. 2009). Additionally, brainstem responses in children with autistic spectrum disorders exhibit a reduction in neural synchrony and phase locking of speech sounds as well as degraded responses by background noise when compared to normal controls (Russo et al. 2009), suggesting that subcortical dysfunction is related to language impairments. The work of the present thesis offers an animal model to study the neuronal correlates of implicit auditory learning and, in combination with genetic models of neurodevelopmental diseases, can contribute to the better understanding of the neuronal deficits underlying higher cognitive processes such as speech acquisition and to the development of therapeutic treatments. 


\section{REFERENCES}

Ahuja, T.K. \& Wu, S.H., 2007. Intrinsic membrane properties and synaptic response characteristics of neurons in the rat's external cortex of the inferior colliculus. Neuroscience, 145(3), pp.85165.

Aitkin, L.M. et al., 1978. External nucleus of inferior colliculus: auditory and spinal somatosensory afferents and their interactions. Journal of neurophysiology, 41(4), pp.837-47.

Aizenberg, M. et al., 2015. Bidirectional Regulation of Innate and Learned Behaviors That Rely on Frequency Discrimination by Cortical Inhibitory Neurons. PLOS Biology, 13(12), p.e1002308.

Aizenberg, M. \& Geffen, M.N., 2013a. Bidirectional effects of aversive learning on perceptual acuity are mediated by the sensory cortex. Nature neuroscience, 16(8), pp.994-6.

Aizenberg, M. \& Geffen, M.N., 2013b. Bidirectional effects of aversive learning on perceptual acuity are mediated by the sensory cortex. Nature neuroscience, 16(8), pp.994-6.

Andersen, R.A., Snyder, R.L. \& Merzenich, M.M., 1980. The topographic organization of corticocollicular projections from physiologically identified loci in the Al, All, and anterior auditory cortical fields of the cat. The Journal of comparative neurology, 191(3), pp.479-94.

Bakin, J.S. \& Weinberger, N.M., 1990. Classical conditioning induces CS-specific receptive field plasticity in the auditory cortex of the guinea pig. Brain Research, 536(1-2), pp.271-286.

Bakin, J.S. \& Weinberger, N.M., 1996. Induction of a physiological memory in the cerebral cortex by stimulation of the nucleus basalis. Proceedings of the National Academy of Sciences of the United States of America, 93(20), pp.11219-11224.

Bao, S., 2015. Perceptual learning in the developing auditory cortex. European Journal of Neuroscience, 41,5,pp.718-724.

Bao, S. et al., 2003. Suppression of cortical representation through backward conditioning. Proceedings of the National Academy of Sciences of the United States of America, 100(3), pp.1405-1408.

Bao, S. et al., 2004. Temporal plasticity in the primary auditory cortex induced by operant perceptual learning. Nature neuroscience, 7(9), pp.974-981.

Bao, S., Chan, V.T. \& Merzenich, M.M., 2001. Cortical remodelling induced by activity of ventral tegmental dopamine neurons. Nature, 412(6842), pp.79-83.

Barkat, T.R., Polley, D.B. \& Hensch, T.K., 2011. A critical period for auditory thalamocortical connectivity. Nature neuroscience, 14(9), pp.1189-94.

Barnstedt, O. et al., 2015. Functional Microarchitecture of the Mouse Dorsal Inferior Colliculus Revealed through In Vivo Two-Photon Calcium Imaging. Journal of Neuroscience, 35(31), pp.10927-10939.

Bednar, J.A. \& Wilson, S.P., 2015. Cortical Maps. The Neuroscientist : a review journal bringing neurobiology, neurology and psychiatry, p.1073858415597645.

Bednarek, E. \& Caroni, P., 2011. $\beta$-Adducin is required for stable assembly of new synapses and improved memory upon environmental enrichment. Neuron, 69(6), pp.1132-46. 
Belelovsky, K. et al., 2005. A molecular switch for translational control in taste memory consolidation. The European journal of neuroscience, 22(10), pp.2560-8.

Benali, A. et al., 2008. Excitation and inhibition jointly regulate cortical reorganization in adult rats. The Journal of neuroscience: the official journal of the Society for Neuroscience, 28(47), pp.12284-93.

Berardi, N., Pizzorusso, T. \& Maffei, L., 2000. Critical periods during sensory development. Current Opinion in Neurobiology, 10(1), pp.138-145.

Bermudez, M.A. \& Schultz, W., 2014. Timing in reward and decision processes. Philosophical transactions of the Royal Society of London. Series B, Biological sciences, 369(1637), p.20120468.

Blake, D.T. et al., 2006. Experience-Dependent Adult Cortical Plasticity Requires Cognitive Association between Sensation and Reward. Neuron, 52(2), pp.371-381.

Blake, D.T. et al., 2002. Neural correlates of instrumental learning in primary auditory cortex. Proceedings of the National Academy of Sciences of the United States of America, 99(15), pp.10114-9.

Bledsoe, S.C., Shore, S.E. \& Guitton, M.J., 2003. Spatial representation of corticofugal input in the inferior colliculus: a multicontact silicon probe approach. Experimental brain research, 153(4), pp.530-42.

Browne, C.J., Morley, J.W. \& Parsons, C.H., 2012. Tracking the expression of excitatory and inhibitory neurotransmission-related proteins and neuroplasticity markers after noise induced hearing loss. PloS one, 7(3), p.e33272.

Bureš, Z. et al., 2014. Acoustical enrichment during early postnatal development changes response properties of inferior colliculus neurons in rats. European Journal of Neuroscience, 40(11), pp.3674-3683.

Butts, D.A. \& Goldman, M.S., 2006. Tuning curves, neuronal variability, and sensory coding. PLoS biology, 4(4), p.e92.

Casseday, J.H., Schreiner, C.E. \& Winer, J.A., 2005. The Inferior Colliculus. In C. E. Winer, Jeffery A Schreiner, ed. The Inferior Colliculus. pp. 626-640.

Chandrasekaran, B. et al., 2009. Context-dependent encoding in the human auditory brainstem relates to hearing speech in noise: implications for developmental dyslexia. Neuron, 64(3), pp.311-9.

Chandrasekaran, B. \& Kraus, N., 2010. The scalp-recorded brainstem response to speech: Neural origins and plasticity. Psychophysiology, 47(2), pp.236-246.

Chandrasekaran, B., Skoe, E. \& Kraus, N., 2014. An integrative model of subcortical auditory plasticity. Brain Topography, 27(4), pp.539-552.

Chang, E.F. \& Merzenich, M.M., 2003. Environmental noise retards auditory cortical development. Science, 300(April), pp.498-502.

Chaudhury, S. \& Wadhwa, S., 2009. Prenatal auditory stimulation alters the levels of CREB mRNA, $\mathrm{p}$-CREB and BDNF expression in chick hippocampus. International journal of developmental neuroscience : the official journal of the International Society for Developmental Neuroscience, 
27(6), pp.583-90.

Chen, I.-W., Helmchen, F. \& Lütcke, H., 2015. Specific Early and Late Oddball-Evoked Responses in Excitatory and Inhibitory Neurons of Mouse Auditory Cortex. The Journal of Neuroscience: The Official Journal of the Society for Neuroscience, 35(36), pp.12560-12573.

Chernock, M.L., Larue, D.T. \& Winer, J. a., 2004. A periodic network of neurochemical modules in the inferior colliculus. Hearing Research, 188(1-2), pp.12-20.

Clause, A., Nguyen, T. \& Kandler, K., 2011. An acoustic startle-based method of assessing frequency discrimination in mice. Journal of Neuroscience Methods, 200(1), pp.63-67.

David, S. V., Fritz, J.B. \& Shamma, S. a., 2012. Task reward structure shapes rapid receptive field plasticity in auditory cortex. Proceedings of the National Academy of Sciences, 109(6), pp.2144-2149.

Diaz, E. et al., 2015. Involvement of D1 and D2 dopamine receptor in the retrieval processes in latent inhibition. Psychopharmacology, 232(23), pp.4337-46.

Donato, F., Rompani, S.B. \& Caroni, P., 2013. Parvalbumin-expressing basket-cell network plasticity induced by experience regulates adult learning. Nature, 504(7479), pp.272-6.

Dong, S. et al., 2010. Tonotopic changes in GABA receptor expression in guinea pig inferior colliculus after partial unilateral hearing loss. Brain Research, 1342, pp.24-32.

Doucet, J.R., Molavi, D.L. \& Ryugo, D.K., 2003. The source of corticocollicular and corticobulbar projections in area Te1 of the rat. Experimental brain research, 153(4), pp.461-6.

Edeline, J.M. \& Weinberger, N.M., 1992. Associative retuning in the thalamic source of input to the amygdala and auditory cortex: receptive field plasticity in the medial division of the medial geniculate body. Behavioral neuroscience, 106(1), pp.81-105.

Egorova, M. et al., 2001. Frequency response areas of neurons in the mouse inferior colliculus. I. Threshold and tuning characteristics. Experimental Brain Research, 140(2), pp.145-161.

Ehret, G. \& Schreiner, C.E., 1997. Frequency resolution and spectral integration (critical band analysis) in single units of the cat primary auditory cortex. Journal of Comparative Physiology - A Sensory, Neural, and Behavioral Physiology, 181(6), pp.635-650.

Ehret, G. \& Schreiner, C.E., 2005. Spectral and intensity coding in the auditory midbrain. The Inferior Colliculus, 74(S110), pp.312-345.

Ehret, Günter Merzenich, M.M., 1985. Auditory midbrain responses parallel spectral integration phenomena. Science (New York, N.Y.), 227(4691), pp.1245-1247.

Engineer, N.D., 2004. Environmental Enrichment Improves Response Strength, Threshold, Selectivity, and Latency of Auditory Cortex Neurons. Journal of Neurophysiology, 92(1), pp.7382.

Faingold, C.L., Boersma Anderson, C.A. \& Caspary, D.M., 1991. Involvement of GABA in acousticallyevoked inhibition in inferior colliculus neurons. Hearing research, 52(1), pp.201-16.

Falkenberg, T. et al., 1992. Increased expression of brain-derived neurotrophic factor mRNA in rat hippocampus is associated with improved spatial memory and enriched environment. Neuroscience letters, 138(1), pp.153-6. 
Fox, J.E., 1979. Habituation and prestimulus inhibition of the auditory startle reflex in decerebrate rats. Physiology \& Behavior, 23(2), pp.291-297.

Froemke, R.C. et al., 2013. Long-term modification of cortical synapses improves sensory perception. Nature neuroscience, 16(1), pp.79-88.

Froemke, R.C., Merzenich, M.M. \& Schreiner, C.E., 2007. A synaptic memory trace for cortical receptive field plasticity. Nature, 450(7168), pp.425-429.

Gabay, Y. et al., 2015. Incidental Auditory Category Learning. Journal of Experimental Psychology: Human Perception and Performance, 41(4), pp.1124-1138.

Gabay, Y. \& Holt, L.L., 2015. Incidental Learning of Sound Categories is Impaired in Developmental Dyslexia. Cortex, 73, pp.131-43.

Galambos, Robert Sheatz, Guy Vernier, V., 1956. Electrophysiological correlates of a conditioned response in cats. Science (New York, N.Y.), 123(3192), pp.376-377.

Gal-Ben-Ari, S. et al., 2012. Consolidation and translation regulation. Learning \& memory (Cold Spring Harbor, N.Y.), 19(9), pp.410-22.

Gao, E. \& Suga, N., 1998. Experience-dependent corticofugal adjustment of midbrain frequency map in bat auditory system. Proceedings of the National Academy of Sciences of the United States of America, 95(21), pp.12663-70.

Gao, E. \& Suga, N., 2000. Experience-dependent plasticity in the auditory cortex and the inferior colliculus of bats: role of the corticofugal system. Proceedings of the National Academy of Sciences of the United States of America, 97(14), pp.8081-8086.

Gierdalski, M., 2001. Rapid Regulation of GAD67 mRNA and Protein Level in Cortical Neurons After Sensory Learning. Cerebral Cortex, 11(9), pp.806-815.

Gittelman, J.X., Perkel, D.J. \& Portfors, C. V., 2013. Dopamine modulates auditory responses in the inferior colliculus in a heterogeneous manner. JARO - Journal of the Association for Research in Otolaryngology, 14(5), pp.719-729.

Glerean, E. et al., 2015. Reorganization of functionally connected brain subnetworks in highfunctioning autism. Human Brain Mapping, p.n/a-n/a.

Gomez-Pinilla, F. et al., 2011. The influence of naturalistic experience on plasticity markers in somatosensory cortex and hippocampus: effects of whisker use. Brain research, 1388, pp.3947.

Gonzalez, G.A. \& Montminy, M.R., 1989. Cyclic AMP stimulates somatostatin gene transcription by phosphorylation of CREB at serine 133. Cell, 59(4), pp.675-80.

Gourévitch, B. et al., 2014. Is the din really harmless? Long-term effects of non-traumatic noise on the adult auditory system. Nature reviews. Neuroscience, 15(7), pp.483-91.

Greifzu, F. et al., 2014. Environmental enrichment extends ocular dominance plasticity into adulthood and protects from stroke-induced impairments of plasticity. Proceedings of the National Academy of Sciences of the United States of America, 111(3), pp.1150-5.

Gubern, C. et al., 2009. Validation of housekeeping genes for quantitative real-time PCR in in-vivo and in-vitro models of cerebral ischaemia. BMC molecular biology, 10(1), p.57. 
Guo, W. et al., 2012. Robustness of Cortical Topography across Fields, Laminae, Anesthetic States, and Neurophysiological Signal Types. Journal of Neuroscience, 32(27), pp.9159-9172.

Hackett, T. a. et al., 2011. Linking Topography to Tonotopy in the Mouse Auditory Thalamocortical Circuit. Journal of Neuroscience, 31(8), pp.2983-2995.

Han, Y.K. et al., 2007. Early experience impairs perceptual discrimination. Nature neuroscience, 10(9), pp.1191-1197.

Hensch, T.K., 2014. Bistable parvalbumin circuits pivotal for brain plasticity. Cell, 156(1-2), pp.1719.

Hensch, T.K. et al., 1998. Local GABA circuit control of experience-dependent plasticity in developing visual cortex. Science (New York, N.Y.), 282(5393), pp.1504-8.

Hequembourg, S. \& Liberman, M.C., 2001. Spiral ligament pathology: a major aspect of age-related cochlear degeneration in C57BL/6 mice. Journal of the Association for Research in Otolaryngology : JARO, 2(2), pp.118-129.

Hofer, S.B. et al., 2009. Experience leaves a lasting structural trace in cortical circuits. Nature, 457(January), pp.313-317.

Holt, A.G. et al., 2005. Deafness-related plasticity in the inferior colliculus: gene expression profiling following removal of peripheral activity. Journal of neurochemistry, 93(5), pp.1069-86.

Holtmaat, A. et al., 2006. Experience-dependent and cell-type-specific spine growth in the neocortex. Nature, 441(7096), pp.979-83.

Holtmaat, A.J.G.D. et al., 2005. Transient and persistent dendritic spines in the neocortex in vivo. Neuron, 45(2), pp.279-91.

Honey, R.C. \& Good, M., 1993. Selective hippocampal lesions abolish the contextual specificity of latent inhibition and conditioning. Behavioral Neuroscience, 107(1), pp.23-33.

De Houwer, J., 2009. The propositional approach to associative learning as an alternative for association formation models. Learning \& behavior : a Psychonomic Society publication, 37(1), pp.1-20.

de Hoz, L. \& Nelken, I., 2014. Frequency tuning in the behaving mouse: different bandwidths for discrimination and generalization. PloS one, 9(3), p.e91676.

$\mathrm{Hu}$, S.S. et al., 2014. Expression of immediate-early genes in the inferior colliculus and auditory cortex in salicylate-induced tinnitus in rat. European Journal of Histochemistry, 58(1), pp.7379.

Ito, T., Bishop, D.C. \& Oliver, D.L., 2009. Two classes of GABAergic neurons in the inferior colliculus. The Journal of neuroscience: the official journal of the Society for Neuroscience, 29(44), pp.13860-13869.

Ito, T. \& Oliver, D.L., 2010. Origins of Glutamatergic Terminals in the Inferior Colliculus Identified by Retrograde Transport and Expression of VGLUT1 and VGLUT2 Genes. Frontiers in neuroanatomy, 4, p.135.

Jeng, F.-C. et al., 2011. Cross-linguistic comparison of frequency-following responses to voice pitch in American and Chinese neonates and adults. Ear and hearing, 32(6), pp.699-707. 
Ji, W., Gao, E. \& Suga, N., 2001. Effects of acetylcholine and atropine on plasticity of central auditory neurons caused by conditioning in bats. Journal of neurophysiology, 86(1), pp.211-25.

Ji, W. \& Suga, N., 2003. Development of reorganization of the auditory cortex caused by fear conditioning: effect of atropine. Journal of neurophysiology, 90(3), pp.1904-1909.

Ji, W., Suga, N. \& Gao, E., 2005. Effects of agonists and antagonists of NMDA and ACh receptors on plasticity of bat auditory system elicited by fear conditioning. Journal of neurophysiology, 94(2), pp.1199-1211.

Kaas, J., 2011. The Auditory Cortex. In M. N. Wallace et al., eds. The Auditory Cortex. Berlin: Springer, pp. 133-145.

Kanold, P.O., Nelken, I. \& Polley, D.B., 2014. Local versus global scales of organization in auditory cortex. Trends in neurosciences, pp.1-9.

Kato, H.K., Gillet, S.N. \& Isaacson, J.S., 2015. Flexible Sensory Representations in Auditory Cortex Driven by Behavioral Relevance. Neuron, 88(5), pp.1027-1039.

Katz, L.C. \& Shatz, C.J., 1996. Synaptic activity and the construction of cortical circuits. Science (New York, N.Y.), 274(5290), pp.1133-8.

Kilgard, M.P. et al., 2007. Experience dependent plasticity alters cortical synchronization. Hearing Research, 229(1-2), pp.171-179.

Kilgard, M.P. \& Merzenich, M.M., 1998. Cortical map reorganization enabled by nucleus basalis activity. Science (New York, N.Y.), 279(5357), pp.1714-8.

Kluge, C. et al., 2011. Plasticity of human auditory-evoked fields induced by shock conditioning and contingency reversal. Proceedings of the National Academy of Sciences of the United States of America, 108(30), pp.12545-50.

Koch, M., 1999. The neurobiology of startle. Progress in Neurobiology, 59(2), pp.107-128.

Kong, L. et al., 2014. Frequency-specific corticofugal modulation of the dorsal cochlear nucleus in mice. Frontiers in Systems Neuroscience, 8(July), pp.1-7.

Kraus, N. \& White-Schwoch, T., 2015. Unraveling the Biology of Auditory Learning: A CognitiveSensorimotor-Reward Framework. Trends in Cognitive Sciences, 19(11), pp.642-654.

Kuhl, P.K., 2010. Brain Mechanisms in Early Language Acquisition. Neuron, 67(5), pp.713-727.

Lai, H., Carino, M.A. \& Wen, Y.-F., 1989. Repeated noise exposure affects muscarinic cholinergic receptors in the rat brain. Brain Research, 488, pp.361-364.

LeBeau, F.E., Malmierca, M.S. \& Rees, A., 2001. Iontophoresis in vivo demonstrates a key role for $\mathrm{GABA}(\mathrm{A})$ and glycinergic inhibition in shaping frequency response areas in the inferior colliculus of guinea pig. The Journal of neuroscience : the official journal of the Society for Neuroscience, 21(18), pp.7303-12.

LeDoux, J.E., Ruggiero, D. a \& Reis, D.J., 1985. Projections to the subcortical forebrain from anatomically defined regions of the medial geniculate body in the rat. The Journal of comparative neurology, 242(2), pp.182-213.

Lee, H.-K. \& Whitt, J.L., 2015. Cross-modal synaptic plasticity in adult primary sensory cortices. 
Current opinion in neurobiology, 35, pp.119-26.

Lee, M.-Y. et al., 2013. Alteration of Synaptic Activity-Regulating Genes Underlying Functional Improvement by Long-term Exposure to an Enriched Environment in the Adult Brain. Neurorehabilitation and Neural Repair, 27(6), pp.561-574.

Li, L. et al., 1998. Prepulse Inhibition Following Lesions of the Inferior Colliculus : Prepulse Intensity Functions. , 65(1), pp.133-139.

Lim, H.H. \& Anderson, D.J., 2007a. Antidromic activation reveals tonotopically organized projections from primary auditory cortex to the central nucleus of the inferior colliculus in guinea pig. Journal of neurophysiology, 97(2), pp.1413-27.

Lim, H.H. \& Anderson, D.J., 2007b. Spatially distinct functional output regions within the central nucleus of the inferior colliculus: implications for an auditory midbrain implant. Journal of Neuroscience, 27(32), pp.8733-8743.

Loftus, W.C. et al., 2004. Organization of binaural excitatory and inhibitory inputs to the inferior colliculus from the superior olive. The Journal of comparative neurology, 472(3), pp.330-44.

Loftus, W.C. et al., 2008. The cytoarchitecture of the inferior colliculus revisited: a common organization of the lateral cortex in rat and cat. Neuroscience, 154(1), pp.196-205.

Loftus, W.C., Bishop, D.C. \& Oliver, D.L., 2010. Differential patterns of inputs create functional zones in central nucleus of inferior colliculus. The Journal of neuroscience : the official journal of the Society for Neuroscience, 30(40), pp.13396-13408.

Lou, Y. et al., 2014. Ventral tegmental area activation promotes firing precision and strength through circuit inhibition in the primary auditory cortex. Frontiers in neural circuits, 8, p.25.

Lovibond, P.F., Preston, G.C. \& Mackintosh, N.J., 1984. Context specificity of conditioning, extinction, and latent inhibition. Journal of Experimental Psychology: Animal Behavior Processes, 10(3), pp.360-375.

Ludwig, K.A. et al., 2009. Using a common average reference to improve cortical neuron recordings from microelectrode arrays. Journal of neurophysiology, 101(3), pp.1679-89.

Luo, F. et al., 2008. Corticofugal modulation of initial sound processing in the brain. The Journal of neuroscience : the official journal of the Society for Neuroscience, 28(45), pp.11615-11621.

Ma, W., Li, Y. \& Tao, H.W., 2013. Downregulation of cortical inhibition mediates ocular dominance plasticity during the critical period. The Journal of neuroscience : the official journal of the Society for Neuroscience, 33(27), pp.11276-80.

Ma, X. \& Suga, N., 2003. Augmentation of plasticity of the central auditory system by the basal forebrain and/or somatosensory cortex. Journal of neurophysiology, 89(1), pp.90-103.

Mainardi, M. et al., 2010. Environmental enrichment potentiates thalamocortical transmission and plasticity in the adult rat visual cortex. Journal of neuroscience research, 88(14), pp.3048-59. Available at: http://www.ncbi.nlm.nih.gov/pubmed/20722076 [Accessed December 21, 2015].

Mainardi, M. et al., 2014. Environmental enrichment strengthens corticocortical interactions and reduces amyloid- $\beta$ oligomers in aged mice. Frontiers in aging neuroscience, 6, p.1. 
Marianowski, R. et al., 2000. Expression of NMDA, AMPA and GABA(A) receptor subunit mRNAs in the rat auditory brainstem. I. Influence of early auditory deprivation. Hearing research, 150(12), pp.1-11. Available at: http://www.ncbi.nlm.nih.gov/pubmed/11077189.

Markovitz, C.D., Tang, T.T. \& Lim, H.H., 2013. Tonotopic and localized pathways from primary auditory cortex to the central nucleus of the inferior colliculus. Frontiers in neural circuits, 7 , p.77.

Martinowich, K. \& Lu, B., 2008. Interaction between BDNF and serotonin: role in mood disorders. Neuropsychopharmacology: official publication of the American College of Neuropsychopharmacology, 33(1), pp.73-83.

Mears, R.P. \& Spencer, K.M., 2012. Electrophysiological assessment of auditory stimulus-specific plasticity in schizophrenia. Biological psychiatry, 71(6), pp.503-11.

Medini, P., 2014. Experience-dependent plasticity of visual cortical microcircuits. Neuroscience, 278, pp.367-84.

Mellott, J.G. et al., 2014. Excitatory and inhibitory projections in parallel pathways from the inferior colliculus to the auditory thalamus. Frontiers in neuroanatomy, 8, p.124.

Melo, L.L. et al., 2009. Effects of microinjections of apomorphine and haloperidol into the inferior colliculus on the latent inhibition of the conditioned emotional response. Experimental Neurology, 216(1), pp.16-21.

Merzenich, M.M., Knight, P.L. \& Roth, G.L., 1975. Representation of cochlea within primary auditory cortex in the cat. Journal of neurophysiology, 38(2), pp.231-249.

Merzenich, M.M. \& Reid, M.D., 1974. Representation of the cochlea within the inferior colliculus of the cat. Brain Research, 77(3), pp.397-415.

Miyakawa, a., Gibboni, R. \& Bao, S., 2013. Repeated exposure to a tone transiently alters spectral tuning bandwidth of neurons in the central nucleus of inferior colliculus in juvenile rats. Neuroscience, 230, pp.114-120.

Montgomery, N. \& Wehr, M., 2010. Auditory cortical neurons convey maximal stimulus-specific information at their best frequency. The Journal of neuroscience : the official journal of the Society for Neuroscience, 30(40), pp.13362-13366.

Morest, D.K. \& Oliver, D.L., 1984. The neuronal architecture of the inferior colliculus in the cat: defining the functional anatomy of the auditory midbrain. The Journal of comparative neurology, 222(2), pp.209-236.

Morris, J.S., Friston, K.J. \& Dolan, R.J., 1998. Experience-dependent modulation of tonotopic neural responses in human auditory cortex. Proceedings. Biological sciences / The Royal Society, 265(1397), pp.649-57.

Moucha, R. et al., 2005. Background sounds contribute to spectrotemporal plasticity in primary auditory cortex. Experimental Brain Research, 162(4), pp.417-427.

Moyer, C.E. et al., 2015. Developmental Trajectories of Auditory Cortex Synaptic Structures and Gap-Prepulse Inhibition of Acoustic Startle Between Early Adolescence and Young Adulthood in Mice. Cerebral cortex (New York, N.Y. : 1991), p.bhv040-.

Mwilambwe-Tshilobo, L. et al., 2015. Selective Impairment in Frequency Discrimination in a Mouse 
Model of Tinnitus. PloS one, 10(9), p.e0137749.

Nakamoto, K.T. et al., 2013. Analysis of excitatory synapses in the guinea pig inferior colliculus: A study using electron microscopy and GABA immunocytochemistry. Neuroscience, 237, pp.170-183.

Nibuya, M., Nestler, E.J. \& Duman, R.S., 1996. Chronic antidepressant administration increases the expression of CAMP response element binding protein (CREB) in rat hippocampus. The Journal of neuroscience : the official journal of the Society for Neuroscience, 16(7), pp.2365-72.

Nichols, J.A. et al., 2007. Environmental enrichment selectively increases glutamatergic responses in layer II/III of the auditory cortex of the rat. Neuroscience, 145(3), pp.832-40.

Nithianantharajah, J. \& Hannan, A.J., 2006. Enriched environments, experience-dependent plasticity and disorders of the nervous system. Nature reviews. Neuroscience, 7(9), pp.697709.

Noreña, A.J. et al., 2006. Spectrally enhanced acoustic environment disrupts frequency representation in cat auditory cortex. Nature Neuroscience, 9(7), pp.932-939.

Norena, A.J. \& Eggermont, J.J., 2005. Enriched Acoustic Environment after Noise Trauma Reduces Hearing Loss and Prevents Cortical Map Reorganization. Journal of Neuroscience, 25(3), pp.699-705.

Oliver, D.L., 2005. Chapter 2 Neuronal Organization in the Inferior Colliculus. In The Inferior Colliculus. pp. 82-83.

Oliver, D.L., Izquierdo, M. a. \& Malmierca, M.S., 2011. Persistent effects of early augmented acoustic environment on the auditory brainstem. Neuroscience, 184, pp.75-87.

Ono, K., Kudoh, M. \& Shibuki, K., 2006. Roles of the auditory cortex in discrimination learning by rats. The European Journal of Neuroscience, 23(6), pp.1623-1632.

Ono, M. \& Ito, T., 2015. Functional organization of the mammalian auditory midbrain. The Journal of Physiological Sciences.

Ortinski, P.I. et al., 2004. Expression of Distinct \{alpha\} Subunits of GABAA Receptor Regulates Inhibitory Synaptic Strength. J.Neurophysiol., .(April 2004), pp.1718-1727.

Palmer, A.R. \& Kuwada, S., 2005. Chapter 13 Binaural and Spatial Coding in the Inferior Colliculus. The Inferior Colliculus, pp.377-410.

Panzeri, S. et al., 2010. Sensory neural codes using multiplexed temporal scales. Trends in Neurosciences, 33(3), pp.111-120.

Percaccio, C.R. et al., 2005. Environmental enrichment increases paired-pulse depression in rat auditory cortex. Journal of neurophysiology, 94(5), pp.3590-600.

Percaccio, C.R. et al., 2007. Sensory experience determines enrichment-induced plasticity in rat auditory cortex. Brain Research, 1174(1), pp.76-91.

Pienkowski, M. \& Eggermont, J.J., 2009. Long-term, partially-reversible reorganization of frequency tuning in mature cat primary auditory cortex can be induced by passive exposure to moderatelevel sounds. Hearing Research, 257(1-2), pp.24-40. 
Polley, D.B. et al., 2004. Associative learning shapes the neural code for stimulus magnitude in primary auditory cortex. Proceedings of the National Academy of Sciences of the United States of America, 101(46), pp.16351-16356.

Polley, D.B., Steinberg, E.E. \& Merzenich, M.M., 2006. Perceptual learning directs auditory cortical map reorganization through top-down influences. The Journal of neuroscience : the official journal of the Society for Neuroscience, 26(18), pp.4970-4982.

Polley, D.B., Thompson, J.H. \& Guo, W., 2013. Brief hearing loss disrupts binaural integration during two early critical periods of auditory cortex development. Nature communications, 4, p.2547.

Poon, P.W.F. \& Chen, X., 1992. Postnatal exposure to tones alters the tuning characteristics of inferior collicular neurons in the rat. Brain Research, 585(1-2), pp.391-394.

Popelář, J., Nwabueze-Ogbo, F.C. \& Syka, J., 2003. Changes in Neuronal Activity of the Inferior Colliculus in Rat after Temporal Inactivation of the Auditory Cortex. Physiological Research, 52(5), pp.615-628.

Portfors, C. V. et al., 2011. Spatial organization of receptive fields in the auditory midbrain of awake mouse. Neuroscience, 193, pp.429-439.

Quian Quiroga, R. \& Panzeri, S., 2009. Extracting information from neuronal populations: information theory and decoding approaches. Nature reviews. Neuroscience, 10(3), pp.173185.

Recanzone, G.H., Schreiner, C.E. \& Merzenich, M.M., 1993. Plasticity in the frequency representation of primary auditory cortex following discrimination training in adult owl monkeys. The Journal of neuroscience : the official journal of the Society for Neuroscience, 13(1), pp.87-103.

Rema, V. et al., 2006. Short exposure to an enriched environment accelerates plasticity in the barrel cortex of adult rats. Neuroscience, 140(2), pp.659-72.

Rubenstein, J.L.R. \& Merzenich, M.M., 2003. Model of autism: increased ratio of excitation/inhibition in key neural systems. Genes, brain, and behavior, 2(5), pp.255-67.

Russo, N. et al., 2009. Brainstem transcription of speech is disrupted in children with autism spectrum disorders. Developmental science, 12(4), pp.557-67.

Russo, N.M. et al., 2008. Deficient brainstem encoding of pitch in children with Autism Spectrum Disorders. Clinical neurophysiology : official journal of the International Federation of Clinical Neurophysiology, 119(8), pp.1720-31.

Rutkowski, R.G. \& Weinberger, N.M., 2005. Encoding of learned importance of sound by magnitude of representational area in primary auditory cortex. Proceedings of the National Academy of Sciences of the United States of America, 102(38), pp.13664-9.

Sale, A., Berardi, N. \& Maffei, L., 2014. Environment and brain plasticity: towards an endogenous pharmacotherapy. Physiological reviews, 94(1), pp.189-234.

Satake, S. et al., 2012. Effects of microinjections of apomorphine and haloperidol into the inferior colliculus on prepulse inhibition of the acoustic startle reflex in rat. Neuroscience letters, 509(1), pp.60-3.

Schreiner, C.E. et al., 1997. Laminar fine structure of frequency organization in auditory midbrain 
the main auditory midbrain station, the central nucleus of the Inferior Colliculus pp.383-386.

Schreiner, C.E. \& Sutter, M.L., 1992. Topography of excitatory bandwidth in cat primary auditory cortex: single-neuron versus multiple-neuron recordings. Journal of neurophysiology, 68(5), pp.1487-502.

Schultz, W., Carelli, R.M. \& Wightman, R.M., 2015. Phasic dopamine signals: from subjective reward value to formal economic utility. Current Opinion in Behavioral Sciences, 5, pp.147-154.

Seybold, B. a. et al., 2012. Chronic reduction in inhibition reduces receptive field size in mouse auditory cortex. Proceedings of the National Academy of Sciences, 109(34), pp.13829-13834.

Skoe, E. \& Chandrasekaran, B., 2014. The layering of auditory experiences in driving experiencedependent subcortical plasticity. Hearing research, 311, pp.36-48.

Skoe, E. \& Kraus, N., 2013. Musical training heightens auditory brainstem function during sensitive periods in development. Frontiers in psychology, 4, p.622.

Slee, S.J. \& David, S. V., 2015. Rapid Task-Related Plasticity of Spectrotemporal Receptive Fields in the Auditory Midbrain. Journal of Neuroscience, 35(38), pp.13090-13102.

Smith, A.R. et al., 2014. Acoustic trauma triggers upregulation of serotonin receptor genes. Hearing Research, 315, pp.40-48.

Stiebler, I. et al., 1997. The auditory cortex of the house mouse: Left-right differences, tonotopic organization and quantitative analysis of frequency representation. Journal of Comparative Physiology - A Sensory, Neural, and Behavioral Physiology, 181(6), pp.559-571.

Stiebler, I. \& Ehret, G., 1985. Inferior colliculus of the house mouse. I. A quantitative study of tonotopic organization, frequency representation, and tone-threshold distribution. J Comp Neurol, 238(1), pp.65-76.

Straka, M.M. et al., 2015. Descending and tonotopic projection patterns from the auditory cortex to the inferior colliculus. Neuroscience, 300, pp.325-37.

Straka, M.M., Schmitz, S. \& Lim, H.H., 2014. Response features across the auditory midbrain reveal an organization consistent with a dual lemniscal pathway. Journal of neurophysiology, 112(4), pp.981-98.

Suga, N. et al., 2002. Plasticity and corticofugal modulation for hearing in adult animals. Neuron, 36(1), pp.9-18.

Suga, N. \& Ma, X., 2003. Multiparametric corticofugal modulation and plasticity in the auditory system. Nature reviews. Neuroscience, 4(10), pp.783-794.

Sutton, M.A. \& Schuman, E.M., 2006. Dendritic protein synthesis, synaptic plasticity, and memory. Cell, 127(1), pp.49-58.

Syka, J. et al., 2000. Response properties of neurons in the central nucleus and external and dorsal cortices of the inferior colliculus in guinea pig. Experimental brain research, 133(2), pp.25466.

Syka, J. \& Popelář, J., 1984. Inferior colliculus in the rat: Neuronal responses to stimulation of the auditory cortex. Neuroscience Letters, 51(2), pp.235-240. 
Taaseh, N., Yaron, A. \& Nelken, I., 2011. Stimulus-specific adaptation and deviance detection in the rat auditory cortex. PloS One, 6(8), p.e23369.

Takamori, S., 2006. VGLUTs: "exciting" times for glutamatergic research? Neuroscience research, 55(4), pp.343-51.

Tan, J. et al., 2007. Tinnitus behavior and hearing function correlate with the reciprocal expression patterns of BDNF and Arg3.1/arc in auditory neurons following acoustic trauma. Neuroscience, 145(2), pp.715-726.

Tao, X. et al., 1998. Ca2+ Influx Regulates BDNF Transcription by a CREB Family Transcription FactorDependent Mechanism. Neuron, 20(4), pp.709-726.

Tardito, D. et al., 2006. Signaling pathways regulating gene expression, neuroplasticity, and neurotrophic mechanisms in the action of antidepressants: a critical overview. Pharmacological reviews, 58(1), pp.115-34.

Tonegawa, S. et al., 2015. Memory Engram Cells Have Come of Age. Neuron, 87(5), pp.918-931.

Tzounopoulos, T. et al., 2004. Cell-specific, spike timing-dependent plasticities in the dorsal cochlear nucleus. Nature neuroscience, 7(7), pp.719-725.

Ulanovsky, N., Las, L. \& Nelken, I., 2003. Processing of low-probability sounds by cortical neurons. Nature Neuroscience, 6(4), pp.391-398.

Vater, M. et al., 1992. The functional role of GABA and glycine in monaural and binaural processing in the inferior colliculus of horseshoe bats. Journal of comparative physiology. A, Sensory, neural, and behavioral physiology, 171(4), pp.541-53.

de Villers-Sidani, E. et al., 2007. Critical period window for spectral tuning defined in the primary auditory cortex (A1) in the rat. The Journal of neuroscience : the official journal of the Society for Neuroscience, 27(1), pp.180-9.

Vlahou, E.L., Protopapas, A. \& Seitz, A.R., 2012. Implicit training of nonnative speech stimuli. Journal of Experimental Psychology: General, 141(2), pp.363-381.

Wang, Y., O'Donohue, H. \& Manis, P., 2011. Short-term plasticity and auditory processing in the ventral cochlear nucleus of normal and hearing-impaired animals. Hearing Research, 279(12), pp.131-139.

Wehr, M. \& Zador, A.M., 2003. Balanced inhibition underlies tuning and sharpens spike timing in auditory cortex. Nature, 426(6965), pp.442-446.

Weinberger, N.M., 2015. New perspectives on the auditory cortex: learning and memory. Handbook of clinical neurology, 129, pp.117-47.

Weinberger, N.M., 2004. Specific long-term memory traces in primary auditory cortex. Nature reviews. Neuroscience, 5(4), pp.279-290.

Whitton, J.P., Hancock, K.E. \& Polley, D.B., 2014. Immersive audiomotor game play enhances neural and perceptual salience of weak signals in noise. Proceedings of the National Academy of Sciences, 111(25), pp.E2606-E2615.

Wilson, S.P. \& Bednar, J.A., 2015. What, if anything, are topological maps for? Developmental Neurobiology, 75(6), pp.667-681. 
Winer, J., 2005. Three systems of descending projections to the inferior colliculus. The Inferior Colliculus, pp.231-247.

Winer, J. a \& Schreiner, C.E., 2005. Chapter 1 The Central Auditory System : A Functional Analysis. The Inferior Colliculus, pp.1-68.

Winer, J.A. et al., 1998. Auditory cortical projections to the cat inferior colliculus. The Journal of comparative neurology, 400(2), pp.147-74.

Wojcik, S.M. et al., 2004. An essential role for vesicular glutamate transporter 1 (VGLUT1) in postnatal development and control of quantal size. Proceedings of the National Academy of Sciences of the United States of America, 101(18), pp.7158-63.

Wong, P.C.M. et al., 2007. Musical experience shapes human brainstem encoding of linguistic pitch patterns. Nature neuroscience, 10(4), pp.420-2.

Wu, G.K. et al., 2008. Lateral sharpening of cortical frequency tuning by approximately balanced inhibition. Neuron, 58(1), pp.132-43.

Wu, Y. \& Yan, J., 2007. Modulation of the receptive fields of midbrain neurons elicited by thalamic electrical stimulation through corticofugal feedback. The Journal of neuroscience : the official journal of the Society for Neuroscience, 27(40), pp.10651-8.

Yan, J. \& Ehret, G., 2002. Corticofugal modulation of midbrain sound processing in the house mouse. European Journal of Neuroscience, 16(1), pp.119-128.

Yan, J. \& Ehret, G., 2001. Corticofugal reorganization of the midbrain tonotopic map in mice. Neuroreport, 12(15), pp.3313-6.

Yang, L., Pollak, G.D. \& Resler, C., 1992. GABAergic circuits sharpen tuning curves and modify response properties in the mustache bat inferior colliculus. Journal of neurophysiology, 68(5), pp.1760-74.

$\mathrm{Yu}, \mathrm{X}$. et al., 2007. Large-scale reorganization of the tonotopic map in mouse auditory midbrain revealed by MRI. Proceedings of the National Academy of Sciences of the United States of America, 104(29), pp.12193-8.

Zeller, K., Rahner-Welsch, S. \& Kuschinsky, W., 1997. Distribution of Glut1 glucose transporters in different brain structures compared to glucose utilization and capillary density of adult rat brains. Journal of cerebral blood flow and metabolism : official journal of the International Society of Cerebral Blood Flow and Metabolism, 17(2), pp.204-9.

Zhang, L.I., Bao, S. \& Merzenich, M.M., 2002. Disruption of primary auditory cortex by synchronous auditory inputs during a critical period. Proceedings of the National Academy of Sciences of the United States of America, 99(4), pp.2309-2314.

Zhang, L.I., Bao, S. \& Merzenich, M.M., 2001. Persistent and specific influences of early acoustic environments on primary auditory cortex. Nature neuroscience, 4(11), pp.1123-30.

Zhang, Y. et al., 2005. Corticofugal feedback for auditory midbrain plasticity elicited by tones and electrical stimulation of basal forebrain in mice. European Journal of Neuroscience, 22(4), pp.871-879.

Zheng, W., 2012. Auditory map reorganization and pitch discrimination in adult rats chronically exposed to low-level ambient noise., 6(September), pp.1-14. 
Zhou, X. et al., 2015. Behavioral training reverses global cortical network dysfunction induced by perinatal antidepressant exposure. Proceedings of the National Academy of Sciences, 112(7), p.201416582.

Zhou, X. et al., 2011. Natural restoration of critical period plasticity in the juvenile and adult primary auditory cortex. The Journal of neuroscience: the official journal of the Society for Neuroscience, 31(15), pp.5625-34.

Zhou, X. \& Merzenich, M.M., 2009. Developmentally degraded cortical temporal processing restored by training. Nature neuroscience, 12(1), pp.26-8.

Zhou, X. \& Merzenich, M.M., 2012. Environmental noise exposure degrades normal listening processes. Nature Communications, 3(May), p.843.

Zhu, X. et al., 2014. Environmental Acoustic Enrichment Promotes Recovery from Developmentally Degraded Auditory Cortical Processing. Journal of Neuroscience, 34(16), pp.5406-5415. 


\section{ACKNOWLEDGMENTS}

I would like to thank my supervisor Livia de Hoz for the opportunity to work in her new lab and for introducing me the fascinating world of auditory physiology and electrophysiology. I cannot imagine other place where I could learn so much and with such a nice harmony and energy. Thank you Livia for your continuous support, the freedom to always try new things and your infinite patience. I am deeply indebted for your support.

I would like to thank Prof. Nave for the opportunity to work in his department, his valuable advices and financial support.

I would also like to thank the other members of my thesis committee: Prof. Moser and Prof. Treue for the fruitful discussions and suggestions throughout the project.

Writing these lines would not be possible without the impressive support of the IMPRS Coordination Office: Prof. Michael Hörner and Sandra Drube. Their help started even before I came to Göttingen. Thank you very much for all the great things you have done for the students of the program.

I want to thank Dr. Strenzke and Dr. Zhing for providing data from the cochlear nucleus and the fruitfult discussions. I also want to thank Dr. Strenzke and Gerhard Hoch for their help with the PPI test.

I want to thank Elena Ciirdaeva, Robert Fledrich and Kathrin Kusch for their support in the technical aspects of gene expression.

I would like to thank Saskia Koehn and Lukas Schad for their help in the gene expression analysis.

I would like to thank the lab members: Sharlen and Chi for the scientific and non-scientific discussions and for their contribution to the nice atmosphere in the lab.

I would like to thank Prof. Israel Nelken for his comments and useful advices for the data analysis.

Finally, I would like to thank my wife Olga Babaev for her constant support in every aspect of my life. The scientific and philosophical discussions with her have profoundly shaped my perspective of science.

This work was partially supported by the Ph.D. program "Neurosciences" - International Max Planck Research School at the Georg August University Göttingen and the GGNB through the Bridging Funding. 


\section{Abbreviations}

$\begin{array}{ll}\text { A1 } & \text { Auditory primary cortex } \\ \text { AAF } & \text { Anterior auditory field } \\ \text { AC } & \text { Auditory cortex } \\ \text { ASR } & \text { Acoustic startle response } \\ \text { BBN } & \text { Broad- band noise } \\ \text { BF } & \text { Best frequency } \\ \text { cABR } & \text { Complex auditory brain stem responses } \\ \text { CAR } & \text { Common average reference } \\ \text { CC } & \text { Cortico-collicular } \\ \text { CF } & \text { Characteristic frequency } \\ \text { CS } & \text { Conditioned stimulus } \\ \text { DC } & \text { Dorsal cortex of inferior colliculus } \\ \text { EAE } & \text { Environmental acoustic enrichment } \\ \text { EAE } & \text { Enriched acoustic environment } \\ \text { ECIC } & \text { External cortex of inferior colliculus } \\ \text { FDA } & \text { Frequency discrimination acuity } \\ \text { FRA } & \text { Frequency response areas } \\ \text { IC } & \text { Inferior colliculus } \\ \text { ICC } & \text { Central nucleus of the inferior colliculus } \\ \text { IP } & \text { Intraperitoneal } \\ \text { LC } & \text { Lateral cortex of inferior colliculus } \\ \text { LI } & \text { Latent inhibition } \\ \text { LPAG } & \text { Periaqueductal gray matter } \\ \text { MGB } & \text { Medial geniculate body } \\ \text { MGBv } & \text { Medial geniculate body of the thalamus } \\ \text { PnC } & \text { Caudal pontine reticular nucleus } \\ \text { PPI } & \text { Pre-pulse inhibition } \\ \text { PPTg } & \text { Pedunculopontine tegmental nucleus } \\ \text { PSTH } & \text { Peri-stimulus time histogram } \\ \text { Th } & \text { Individual discrimination thresholds } \\ \text { US } & \text { Unconditioned stimulus } \\ \text { VTA } & \text { Ventral tegmental area } \\ & \\ \text { IP }\end{array}$




\section{List of figures}

Figure 1.1: Frequency representation in the auditory cortex ................................................. 5

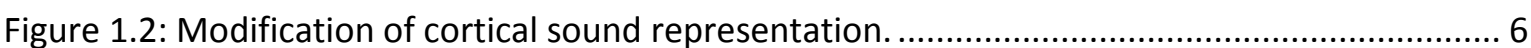

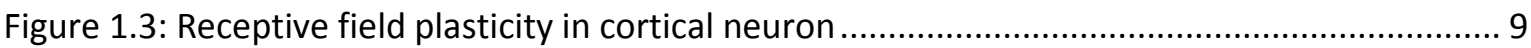

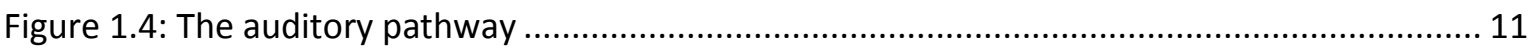

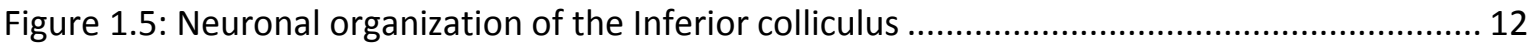

Figure 1.6: Frequency representation in the inferior colliculus of the mouse ............................... 13

Figure 1.7: Cortico-collicular projections ...................................................................................... 14

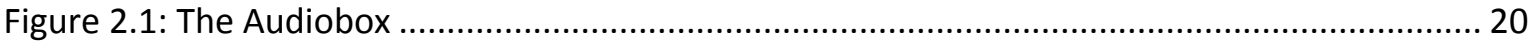

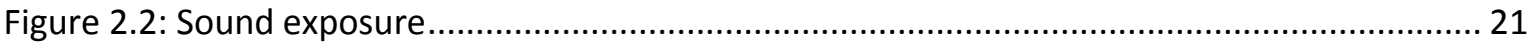

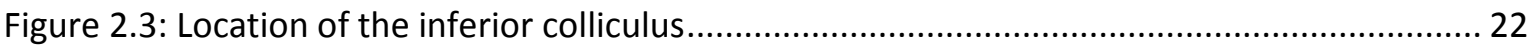

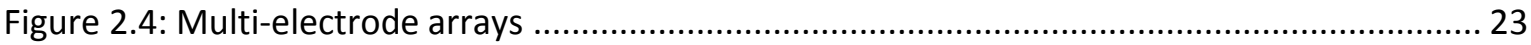

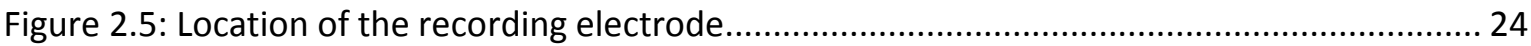

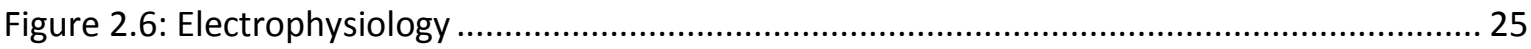

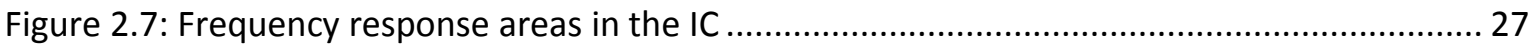

Figure 2.8: Pre-pulse inhibition of the acoustic startle response ............................................... 33

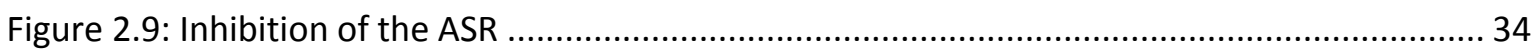

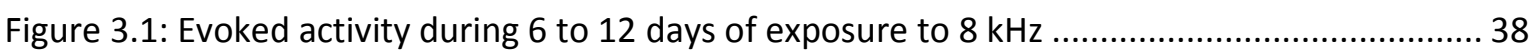

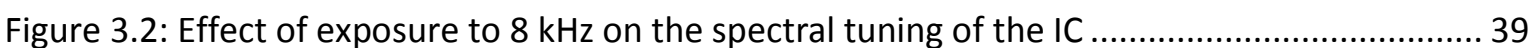

Figure 3.4: Effect of exposure to $16 \mathrm{kHz}$ on the spectral tuning of the IC ..................................... 41

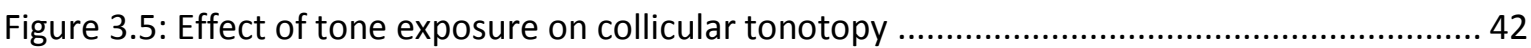

Figure 3.6: Effect of tone exposure on the spontaneous activity in the IC................................... 45

Figure 3.7: Effect of tone exposure on the firing pattern of the IC................................................. 46

Figure 3.8: Effect of relevant tone exposure on the spatio-temporal firing pattern to $8 \mathrm{kHz}$ in the IC

Figure 3.9: Effect of relevant tone exposure on the spatio-temporal firing pattern to $16 \mathrm{kHz}$ in the IC......

Figure 3.10: Effect of relevant and random tone exposure on the spatio-temporal firing pattern to $8 \mathrm{kHz}$ in the IC

Figure 3.11: Effect of relevant and random tone exposure on the spatiotemporal firing pattern to $16 \mathrm{kHz}$ in the IC.

Figure 3.12: Effect of tone exposure on the tonotopic organization at the rostro-caudal and dorsoventral axes of the IC. 
Figure 3.14: Effect during the initial days of tone exposure on the spectral tuning of the IC 56

Figure 3.15: Effect of time of sound exposure on collicular tonotopy

Figure 3.16: Effect of time of sound exposure on collicular tonotopy for the control and the homecage groups 58

Figure 3.17: Inactivation of auditory cortex with muscimol 60

Figure 3.18: Effect of cortical inactivation during the early phase of tone exposure on the spectral tuning of the IC.

Figure 3.19: Effect of cortical inactivation during the late phase of tone exposure on the spectral tuning of the IC.

Figure 3.20: Effect of cortical inactivation on collicular tonotopy

Figure 3.21: Evoked activity in the lateral cortex of the IC during the early and late phases of tone exposure. 65

Figure 3.22: Effect during the early and late phases of tone exposure on the spectral tuning of the lateral cortex of the IC.. 66

Figure 3.23: Gene expression in the IC of the housekeeping gene rpl13a 67

Figure 3.24: Effect of tone exposure on the collicular gene expression of immediate early genes 70 Figure 3.25: Effect of tone exposure on the collicular gene expression of genes involved in inhibitory transmission.

Figure 3.26: Effect of tone exposure on the collicular gene expression of genes involved in excitatory transmission.

Figure 3.27: Effect of tone exposure on the collicular gene expression of genes involved in neuronal plasticity.

Figure 3.28: Effect of tone exposure on the collicular vglut2/vgat expression ratio.

Figure 3.29: Effect of tone exposure on the cortical gene expression of genes involved in neuronal plasticity.

Figure 3.30: Effect of tone exposure on the collicular expression of presynaptic proteins.

Figure 3.31: Effect of tone exposure on sound processing in the cochlear nucleus. .75

Figure 3.32: Effect of tone exposure on frequency discrimination acuity

Figure 3.33: Effect of tone exposure during the pre-conditioned phase on the expression of latent inhibition

Figure 3.34: Effect of conditioning on collicular tonotopy

Figure 3.35: Effect of conditioning on the responses to the conditioned tone (16 kHz) in the IC... 82

Figure 3.36: Effect of conditioning on the responses to the safe tone $(8 \mathrm{kHz})$ in the IC .83

Figure 3.37: Effect of conditioning on the firing pattern along the rostro-caudal axis of the IC..... 84 
Figure 3.38: c-Fos expression increase in auditory cortex following sound discrimination training is blocked by lentivirally delivered shRNA against c-fos 88

Figure 3.39: Blocking c-Fos expression in auditory cortex impairs experience-dependent plasticity 89

Figure 3.40. Blocking c-Fos expression in auditory cortex impairs sound discrimination learning . 90 Figure 4.1: Correlation between two behavioral measures of frequency discrimination and the shift in the tonotopic map caused by relevant and random tone exposure

\section{List of tables}

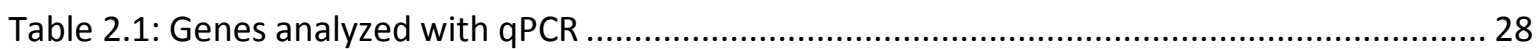

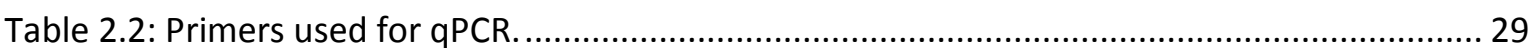

Table 3.1: Effect of sound exposure on collicular gene expression ................................................ 69 
Herewith I declare, that I prepared the PhD Thesis Neuronal correlates of implicit learning in the mammalian midbrain on my own and with no other sources and aids than quoted.

Göttingen, February $29^{\text {th }}, 2016$ Hugo Cruces Solís 


\title{
CURRICULUM VITAE
}

\author{
Name: \\ Hugo Cruces Solís \\ Date and place of birth: \\ October $3^{\text {rd }}, 1983$; Mexico City \\ Nationality: \\ Mexican \\ E-mail address: cruces@em.mpg.de \\ Current address: Herman-Reinn-Str. 5, 37075 Göttingen, Germany.
}

\section{EDUCATION}

Oct 2011- present. Graduate student in the International Max Planck Research School Master's/PhD/MD-PhD Neuroscience Program and the Göttingen Graduate School of Neurosciences and Molecular Biosciences (GGNB) from the Georg- August-Universität, Göttingen. MasterGöttingen, Germany.

Aug 2009- Sept 2011. Master of Science (Neurobiology). National Autonomous University of Mexico. Neurobiology Institute. Mexico.

Nov 2000 - Oct 2008. Bachelor of Science (Biomedical Basic Research). National Autonomous University of Mexico. Faculty of Medicine. Mexico.

\section{PUBLICATIONS}

de Hoz L, Gierej D, Lioudyno V, Jaworski J, Blazejczyk M, Cruces-Solís H, Suska A, Lebitko T, Nikolaev T, Knapska E, Nelken I, Kaczmarek L. (2016) Blocking c-Fos expression reveals the role of auditory cortex plasticity in sound frequency discrimination learning. under revision

Hernández-Hernádez H, Cruces-Solis H, Elías-Viñas D, Verdugo-Díaz L. (2010) Neurite Outgrowth on Chromaffin Cells Applying Extremely Low Frequency Magnetic Fields by Permanent Magnets. Arch Med Res 40:7 545-550.

\section{HONORS AND SCHOLARSHIPS}

2015: Graduate student Travel Award, ARO MidWinter Meeting.

2011: Graduate with honors from MSc.

2011 - 2012: Stipend of the Excellence Foundation for the Promotion of the Max Planck Society.

2009 - 2011: CONACYT Scholarship.

2001- 2004: PRONABES Scholarship.

\section{MEETINGS}

Cruces-Solis H, Zing J, Strenzke N, de Hoz L. "Latent learning induces plastic reorganization of sound representations in the adult midbrain". EBBS - EBPS Joint Meeting 2015. Italy. Oral presentation.

Cruces-Solis H, Zing J, Strenzke N, de Hoz L. "Meaningful sound exposure induces long lasting and spatially-restricted inferior colliculus". $11^{\text {th }}$ Göttingen Meeting of the German Neuroscience Society 2015. Germany. Poster. 
Cruces-Solis H, Zing J, Strenzke N, de Hoz L. "Meaningful but passive sound exposure induces long lasting and spatially-restricted plasticity in adult inferior colliculus." ARO 39th Midwinter Meeting 2015. USA. Poster.

Cruces-Solis H, Zing J, Strenzke N, de Hoz L. "Tone exposure without conditioning modifies sound processing in mouse inferior colliculus". $9^{\text {th }}$ FENS Forum of Neuroscience 2014. Italy. Poster.

Cruces-Solis $\mathrm{H}$ and de Hoz L. "Tone exposure without conditioning modifies sound processing in mouse inferior colliculus" PhD Retreat Max Planck for Experimental Medicine 2014. Germany. Poster. *Winner of best poster.

Cruces-Solis $\mathrm{H}$ and de Hoz L. "Differential experience-dependent plasticity in mouse inferior colliculus depending on prior exposure". 10 $0^{\text {th }}$ Göttingen Meeting of the German Neuroscience Society. Germany 2013.2 Poster.

Stodieck SK, Goetze B, Greifzu F, Cruces-Solis H, Schmidt KF, Löwel S. "Dark exposure rescues ocular dominance plasticity in the visual cortex of adult mice in an age-dependent manner". $10^{\text {th }}$ Göttingen Meeting of the German Neuroscience Society 2013. Germany.

Moreno-Carranza B, Vega C, Lopez-Barrera F, Nava G, Cruces-Solis H, Quintanar-Stephano A, Binart $\mathrm{N}$, Martínez de la Escalera $\mathrm{G}$ and Clapp C. "Prolactin promotes liver regeneration after partial hepatectomy" 93rd Annual Meeting of the Endocrinolgy Society 2011. U.S.A. Poster.

*Winner of the ENDO 2011 Presidential Poster Competition.

Cruces-Solis H, Hernández-Hernádez H, Elías-Viñas D, Verdugo-Díaz L. "Neurite-like outgrowth in chromaffin cells induced by extreme-low frequency magnetic field stimulation. Dependence on frequency. Society for Neuroscience Annual Meeting 2006. USA. Poster.

\section{COURSES AND OTHER ACTIVITIES}

Organizing member of the $6^{\text {th }}$ Neurizons Biennial Neuroscience Conference of the International Max Planck Research School for Neuroscience. 2015. Göttingen. Germany. Head of speakers invitations. Speakers invited: György Buzsáki, Wolfram Schultz, Miguel Nicolelis, Wolf Singer, Rodrigo Quiroga.

Organizing member of the $5^{\text {th }}$ Neurizons Biennial Neuroscience Conference of the International Max Planck Research School for Neuroscience. 2013. Göttingen. Germany.

"Assessment of rodents behavior: methods and rationale. Symposium in Helsinki". 2012. Finland. 
